\title{
Soft X-ray stereo microscopy for investigation of dynamics and elemental distribution of colloidal systems from the environment
}

\author{
Dissertation \\ zur Erlangung des Doktorgrades \\ der Mathematisch-Naturwissenschaftlichen Fakultäten \\ der Georg-August-Universität zu Göttingen
}

vorgelegt von

Sophie-Charlotte Gleber

aus Wolfenbüttel

Göttingen 2009 
Referent:

Korreferent:

Tag der mündlichen Prüfung: 20.03.2009
Prof. Dr. T. Salditt

Prof. Dr. G. Marowsky 


\section{Contents}

1 Introduction 1

2 Soft X-ray microscopy 3

2.1 Image formation . . . . . . . . . . . . . . . . . . 3

2.2 Zone plates as soft X-ray optics . . . . . . . . . . . . . . 6

2.2.1 Depth of focus . . . . . . . . . . . . . 7

2.3 Absorption contrast . . . . . . . . . . . . . . . 11

2.3.1 Radiation damage . . . . . . . . . . . . . . 13

2.4 Spectromicroscopy . . . . . . . . . . . . . . . . . 14

2.4.1 Absorption spectra . . . . . . . . . . . . . . . 14

2.4 .2 Elemental mapping . . . . . . . . . . . . . . . . . . . . 17

3 Stereo microscopy $\quad 19$

3.1 Sample holders . . . . . . . . . . . . . . . . . 20

3.2 Stereo reconstruction . . . . . . . . . . . . . . . . . . . . 24

3.2.1 Spatial coordinates calculated from parallax equation . . . . . . . 24

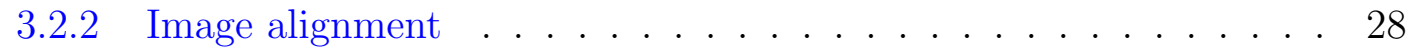

3.2 .3 Rotation axis . . . . . . . . . . . . . . . 30

3.2.4 Resolution limit . . . . . . . . . . . . . . . . . . . 32

3.3 Programme xstereo . . . . . . . . . . . . . . . . . . 33 
4 Soft X-ray microscopes $\quad 39$

4.1 Full-field soft X-ray microscopes . . . . . . . . . . . . . . . . . . 39

4.1 .1 XM-1 at ALS . . . . . . . . . . . . . . . . . . 39

4.1.2 Compact soft X-ray microscope at KTH . . . . . . . . . . . . . . 42

4.2 Scanning soft X-ray microscopes . . . . . . . . . . . . . . . . . 44

4.2 .1 STXM at BESSY . . . . . . . . . . . . . . 44

4.2 .2 TWINMIC at ELETTRA . . . . . . . . . . . . . . . . . . 48

5 Samples from environmental science $\quad 51$

5.1 Inorganic soil fraction . . . . . . . . . . . . . . . . . . . . 52

5.1 .1 Examples for clay minerals . . . . . . . . . . . . . . . . 56

5.1 .2 Iron oxides . . . . . . . . . . . . . . . . . . . . . 57

5.2 Organic soil fraction . . . . . . . . . . . . . . . . . 58

5.3 Soil colloids . . . . . . . . . . . . . . . . . . . . . . 59

5.3 .1 Interactions of colloids . . . . . . . . . . . . . . . 60

5.3 .2 Point of zero charge . . . . . . . . . . . . . . . . . 62

5.4 Examples for soils . . . . . . . . . . . . . . . . . . . . . . . 65

5.5 Anthropogenically induced substances . . . . . . . . . . . . . . 65

5.5.1 Detergents ....................... 65

5.5.2 Carbon nanotubes . . . . . . . . . . . . . . 66

$\begin{array}{lll}6 & \text { Experimental results } & 67\end{array}$

6.1 Haematite and goethite . . . . . . . . . . . . . . . 68

6.1 .1 Iron mapping . . . . . . . . . . . . . . . . . . . . 71

6.2 Carbon nanotubes . . . . . . . . . . . . . . . . 73

6.3 Kaolinite . . . . . . . . . . . . . . . . . . . . 75

6.3.1 Kaolinite with haematite . . . . . . . . . . . . . . 76 
6.4 Nontronite . . . . . . . . . . . . . . . . . . . . . . . . . . . . 79

6.4.1 Nontronite with haematite . . . . . . . . . . . . . . . 79

6.5 Montmorillonite . . . . . . . . . . . . . . . . 83

6.5.1 Montmorillonite with haematite . . . . . . . . . . . . 84

6.5.2 Montmorillonite with carbon nanotubes . . . . . . . . . . . 90

6.5.3 Montmorillonite with detergent . . . . . . . . . . . . 102

6.6 Calcaric phaeozem . . . . . . . . . . . . . . . . . . 104

6.6.1 Phaeozem with haematite . . . . . . . . . . . . . . 108

6.6.2 Humic substances extracted from phaeozem with haematite . . . 111

6.7 Orthic luvisol . . . . . . . . . . . . . . . . . . . . . 112

6.7.1 Orthic luvisol with haematite . . . . . . . . . . . . . 112

6.8 Dystric planosol . . . . . . . . . . . . . . . . . . . . . . . 116

6.8.1 Dystric planosol with haematite . . . . . . . . . . . . 118

6.9 Influence of $\mathrm{pH} \ldots \ldots \ldots \ldots$. . . . . . . . . . . . . . . . . . . . . . .

7 Conclusion and Outlook $\quad 123$

$\begin{array}{lr}\text { Bibliography } & 127\end{array}$ 


\section{List of Figures}

2.1 Schematic image formation according to Abbé . . . . . . . . . . . . . 4

2.2 Schematic of a Fresnel zone plate . . . . . . . . . . . . . . . . . . . 6

2.3 Different definitions for depth of focus . . . . . . . . . . . . . . 8

2.4 Intensity distribution for illumination with both monochromatic and narrow bandwidth radiation . . . . . . . . . . . . . . . . . . 10

2.5 Plot of the modulation transfer function as a function of spatial frequency of focused and defocused object positions for both monochromatic and narrow bandwidth radiation . . . . . . . . . . . . . . . . . . . 10

2.6 Schematic of electron transition by photon absorption and K-shell absorption spectrum . . . . . . . . . . . . . . . . . 15

2.7 Linear absorption coefficients of water, phenol, and smectite as a function of energy . . . . . . . . . . . . . . . . . . . . . . 15

2.8 Spectra from elemental iron and single valent iron minerals around the L3 and L2 absorption edges of iron . . . . . . . . . . . . . . . . . . 16

3.1 Tiltable object holders . . . . . . . . . . . . . . . . . . 21

3.2 Schematic of a coated $\mathrm{Si}_{3} \mathrm{~N}_{4}$ membrane with channel structure . . . . . . 23

3.3 Fluidic cell sample holder . . . . . . . . . . . . . . . . . . . . . . . 24

3.4 Anaglyph image from X-ray stereo micrographs . . . . . . . . . . . 25

3.5 Schematic of tilt of two-dimensional object . . . . . . . . . . . . 25

3.6 Schematic of tilt of three-dimensional object . . . . . . . . . . . . 26

3.7 xstereo user interface . . . . . . . . . . . . . . . . . . . . 34

3.8 Plot tool "M_Xplot3d" for xstereo . . . . . . . . . . . . . . 36

4.1 Scheme of the X-ray microscope XM-1 beamline at the ALS . . . . . . 40

4.2 Photograph of the X-ray microscope XM-1 at the ALS . . . . . . . . . . 40

4.3 Scheme of the compact soft X-ray microscope at the KTH . . . . . . . . 42

4.4 Photograph of the Compact soft X-ray microscope (CSXM) at the KTH 43

4.5 Scheme of the the U 41 beamline at BESSY . . . . . . . . . . . . . . 45 
4.6 Schematic of the Scanning Transmission X-ray microscope at BESSY . . 45

4.7 Photographs of the STXM object stage between two vacuum vessels with insertable visible light microscope . . . . . . . . . . . . . . . 47

4.8 Photographs of the STXM object holder plate modified for stereo imaging and the detachable object holder . . . . . . . . . . . . . . 47

4.9 Scheme of the $1.1 \mathrm{~L}$ beamline at ELETTRA . . . . . . . . . . . . . 49

4.10 Photograph of the TwinMic . . . . . . . . . . . . . . . . 50

5.1 Structures of two-layer and three-layer clay . . . . . . . . . . . . . 54

5.2 Change of edge charge dependent on $\mathrm{pH}$ value . . . . . . . . . . 63

5.3 Plot of surface charge density against $\mathrm{pH}$ value . . . . . . . . . . . . . 64

6.1 Aqueous haematite particles . . . . . . . . . . . . . . . . . . 69

6.2 Aqueous goethite particles, haematite dispersion induced . . . . . . . . 70

6.3 Goethite dispersion with haematite analysed with xstereo . . . . . . . 71

6.4 Haematite mapping within a montmorillonite sample, four different energies 72

6.5 Aqueous carbon nanotubes (baytubes) imaged at $396 \mathrm{eV}$ and $300 \mathrm{eV}$. . 74

6.6 Micrographs of dried carbon nanotubes (rCNT) taken at $282 \mathrm{eV}, 288.5 \mathrm{eV}$, and $300 \mathrm{eV} \ldots \ldots \ldots \ldots \ldots \ldots$

6.7 Aqueous kaolinite dispersion in a capillary at $707 \mathrm{eV}$. . . . . . . . 76

6.8 Haematite mapping in kaolinite sample . . . . . . . . . . . . . . . . . . 77

6.9 Morphological changes within an aqueous kaolinite dispersion in a capil-

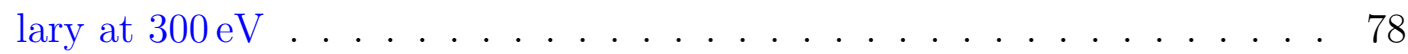

6.10 Micrographs of nontronite around the Fe L absorption edge and resulting iron distribution map . . . . . . . . . . . . . . . . 80

6.11 Pair of stereo images of an aqueous nontronite sample . . . . . . . . . 81

6.12 Pair of stereo images of an aqueous nontronite sample with haematite . . 81

6.13 xstereo analysis of an aqueous nontronite sample with haematite . . . . 82

6.14 Stereo images of aqueous sample of montmorillonite . . . . . . . . . . 83

6.15 Stereo images of aqueous sample of montmorillonite . . . . . . . . . . . . 84

6.16 Stereo micrographs with control image of aqueous sample of montmorillonite 85

6.17 Haematite mapping within an aqueous sample of montmorillonite . . . . 86

6.18 Structures of previously shown aqueous sample of montmorillonite mixed with haematite marked for stereo analysis . . . . . . . . . . . . . . . 87

6.19 Structures of an aqueous sample of montmorillonite mixed with haematite marked for stereo analysis . . . . . . . . . . . . . . . . 87 
6.20 Addition of haematite to an aqueous sample of montmorillonite . . . . . 89

6.21 Stereo images of aqueous sample of montmorillonite . . . . . . . . . . . 90

6.22 Identification of sample movement during the stereo experiment shown in figure 6.21 by single images . . . . . . . . . . . . . . . . . . . . 92

6.23 xstereo processing of stereo micrographs shown in figure $6.21 \ldots$. . . . 96

6.24 Stereo images of aqueous sample of montmorillonite . . . . . . . . . . . . 97

6.25 xstereo processing of stereo micrographs shown in figure 6.24 . . . . . 98

6.26 Elemental mapping of carbon nanotubes within a dried montmorillonite sample . . . . . . . . . . . . . . . . . . . . . . 99

6.27 Elemental mapping of carbon nanotubes within a dried montmorillonite sample . . . . . . . . . . . . . . . . . . . 100

6.28 Micrographs of dried montmorillonite with carbon nanotubes at $282 \mathrm{eV}$

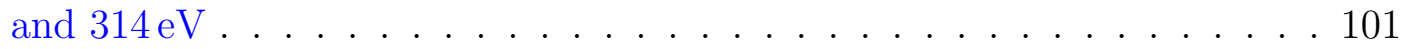

6.29 Addition of carbon nanotubes to an aqueous sample of montmorillonite . 102

6.30 Two micrographs of aqueous montmorillonite mixed with detergent (DTB) in different ratios and corresponding xstereo plots . . . . . . . . . . 103

6.31 Micrographs of phaeozem taken at the Fe L absorption edge . . . . . . . 105

6.32 Structural changes within an aqueous phaeozem sample shown in nine image sequences . . . . . . . . . . . . . . . . . . 106

6.33 xstereo processing of a calcaric phaeozem dispersion in a capillary . . . 107

6.34 Haematite mapping in a mixed calcaric phaeozem sample . . . . . . . . . 109

6.35 Stereo micrographs of aqueous calcaric phaeozem, progression after addition of haematite . . . . . . . . . . . . . . . . . . . 110

6.36 xstereo processing of aqueous phaeozem . . . . . . . . . . . . . . 110

6.37 Micrographs of humic substances from phaeozem with haematite around Fe L absorption edge . . . . . . . . . . . . . . . . . . . . . . . . 111

6.38 Stereo images of aqueous orthic luvisol mixed with haematite . . . . . . . 113

6.39 Single micrographs of the previous sample (figure 6.38) to identify particle movement . . . . . . . . . . . . . . . . . . . . . . 114

6.40 Iron detection in a dried dystric planosol sample . . . . . . . . . . . . . 116

6.41 Micrograph of aqueous dystric planosol . . . . . . . . . . . . . . . . 117

6.42 Stereo images of aqueous sample of planosol mixed with haematite . . . . 118

6.43 Sequences of stereo images shown in figure 6.42 . . . . . . . . . . . . 119 


\section{List of Tables}

2.1 X-ray transmission at various energies of different glass capillaries and stacks of $\mathrm{Si}_{3} \mathrm{~N}_{4}$ membranes filled with water . . . . . . . . . . . 13

5.1 Grain sizing of fine soil particles . . . . . . . . . . . . . . . 52

5.2 Classification of sheet silicates by sheet charge . . . . . . . . . . . . 55

5.3 Point of zero charge of relevant clays and iron oxides . . . . . . . . . . . 64 


\section{Chapter 1}

\section{Introduction}

The world of the colloids is not fully understood yet (Hofmann et al., 2003 (30)) and therefore also referred to as "world of the neglected dimensions" (Brezesinski und Mögel, $1993(10))$. Colloids are defined to be particles with a characteristic length smaller than $1 \mu \mathrm{m}$. They have a huge surface to volume ratio, so the surface characteristic is a crucial feature of soil colloids and the interactions between colloids differ from the interactions of bulk matter. These colloidal interactions are not fully understood yet.

For the investigation of the colloidal interactions, the systems have to be kept in their natural aqueous environment, so the colloidal particles maintain their original shape and interactions like aggregation or adsorption can occur.

It has been shown that soft X-ray microscopy is very well suited for imaging aqueous colloidal systems (for example Thieme et al., 1992 (85), Thieme and Niemeyer, 1998 (87), Niemeyer et al., 1998 (58)). The spatial resolution of $20 \mathrm{~nm}$ to $50 \mathrm{~nm}$ matches with the colloidal size range, and due to the advantageous absorption features in the soft X-ray energy range, water layers of up to $10 \mu \mathrm{m}$ thickness can be penetrated while a natural contrast is given for soil colloids. Thus, high resolution micrographs of soil samples in aqueous media can be obtained.

X-ray microscopy provides also high spectral resolution, allowing for spectromicroscopic investigations of aqueous soil samples (Thieme et al., 2007 (86), Thieme et al., $2007(84)$ ). It is possible to obtain absorption spectra and elemental distribution maps.

However, for the investigation of colloidal interactions, three-dimensional distances have to be revealed. This can be done by soft X-ray tomography (Knöchel et al., 2003 (41)), but cryo fixation is necessary to avoid detectable radiation damage (Schneider, 1992 (66) and Schneider, 1999 (67)). 
Therefore, the combination of high resolution chemical-sensitive soft X-ray microscopy with stereo imaging and processing techniques presented here forms a novel tool for the investigation of aqueous colloidal systems. Information about the spatial distribution within the sample is provided with small calculation effort processing just a pair of stereo micrographs. Thus, the extension towards investigation of dynamical behaviour is possible on the part of the experiment as well as of the processing. 


\section{Chapter 2}

\section{Soft X-ray microscopy}

Soft X-ray microscopy has become a mature analytical tool with numerous applications to nanoscale structures in materials, environmental and biological sciences. A compendium of the latest achievements can be found in the conference proceedings of the X-ray microscopy conference 2002 (Susini et al, 2004 (81)), 2005 (Aoki et al., 2007 (3)), and 2008 (Quitmann et al, 2009 (61)). Recording the X-ray images within the so-called water window, i.e. using photon energies between the K-absorption edges of oxygen $(\mathrm{E} \approx 530 \mathrm{eV}$ or $\lambda=2.34 \mathrm{~nm})$ and carbon $(\mathrm{E} \approx 280 \mathrm{eV}$ or $\lambda=4.43 \mathrm{~nm})$, is especially suitable for imaging samples in aqueous media (Wolter, 1952 (98)). A reasonable optical thickness for imaging in transmission is determined by the 1 /e ratio of the transmitted radiation, which in the water window (Wolter, 1952 (98)) extends up to $10 \mu \mathrm{m}$ around the oxygen K-edge (Kirz et al., 1995 (39)). Since spatial resolution scales with the wavelength, soft X-ray microscopy enables high resolution transmission images of much higher spatial resolution compared to conventional optical microscopy. Currently, the best spatial resolution achieved in soft X-ray microscopy is $15 \mathrm{~nm}$ (Chao et al., 2005 (12)). The challenges for soft X-ray microscopy are the disposition of brilliant light sources with small emittance, the fabrication of highly efficient, nano structured diffractive optics, and the minimisation of radiation damage of X-ray sensitive samples.

\subsection{Image formation}

The image formation by Abbé treats a coherent light source and assumes that the diffraction of the imaging light is caused exclusively by the object structure (Born and Wolf, 1980 (9)). As depicted in the schematic shown in figure 2.1, the light source is assumed to be a point source emitting spherical waves. With the demand of at least 2 orders, the image is formed by the interference of e.g. $0^{\text {th }}$ and $1^{\text {st }}$ order at the image 

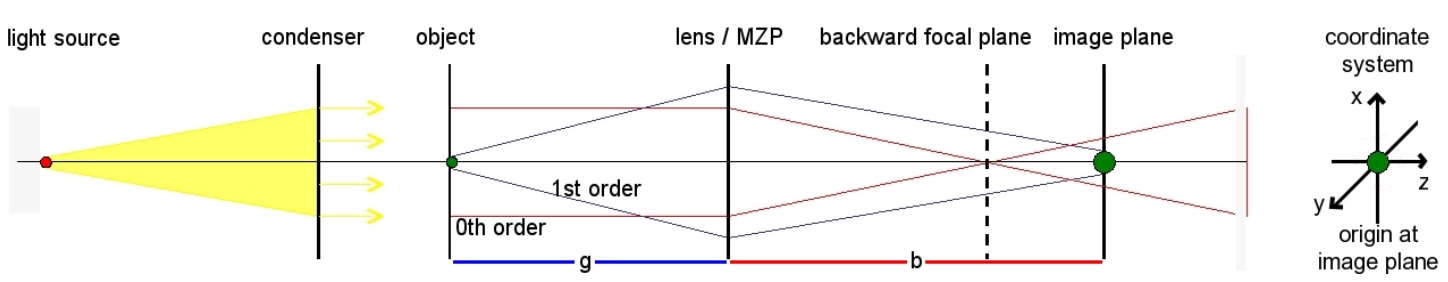

Fig. 2.1: Schematic image formation according to Abbé using a point source for illumination.

plane. The backward focal plane can be used for further image filtering, for example by introducing a phase shifting pattern.

The image of a point created by a rectangular diffrative optic is described as:

$$
I(v)=\left(\frac{\sin v}{v}\right)^{2} I_{0}
$$

where $v$ gives the position in the $\mathrm{x}-\mathrm{y}$-plane.

The image of a point created by a circular aperture is derived as follows (according to Born and Wolf, 1980 (9)): The three-dimensional intensity distribution is given by

$$
I(u, v)=\left(\sin \frac{2}{u}\right)^{2}\left(U_{1}^{2}(u, v)+U_{2}^{2}(u, v)\right) I_{0}
$$

where $u$ gives the position in z-direction and $v$ the position in the $\mathrm{x}-\mathrm{y}$-plane in cylindrical coordinates:

$$
u=\frac{2 \pi}{\lambda}\left(\frac{a}{f}\right)^{2} z \quad \text { and } \quad v=\frac{2 \pi}{\lambda}\left(\frac{a}{f}\right) \sqrt{x^{2}+y^{2}}
$$

with wavelength $\lambda$, lens radius $a$, and focal length $f$. The origin in $z$ is at the image plane $(u=z=0)$. $U_{1}, U_{2}$ are the Lommel functions

$$
U_{n}(u, v)=\sum_{s=0}^{\infty}(-1)^{s}\left(\frac{u}{v}\right)^{n+2 s} J_{n+2 s}(v)
$$

where $J_{n+2 s}(v)$ is the Bessel functions of $\mathrm{n}^{\text {th }}$ order. Thus, the intensity distribution of a luminous point becomes at the image plane $(u=z=0)$

$$
I(0, v)=\left(\frac{2 J_{n}(v)}{v}\right)^{2} I_{0}
$$

This is the Airy formula for Fraunhofer diffraction at a circular aperture. The initial intensity $I_{0}$ is given by

$$
I_{0}=\left(\frac{\pi a^{2}|A|}{\lambda f^{2}}\right)^{2}
$$


with the amplitude $A$ of the initial wave. It scales with $1 / f^{2}$ due to the divergence of the spherical waves. Along the optical axis, the image distribution is given by

$$
I(u, 0)=\left(\frac{\sin (u / 4)}{(u / 4)}\right)^{2} I_{0}
$$

Due to the Babinet's theorem, the conclusions for an intensity distribution as an image of a self-luminous point can be assumed for the image of an absorbing point (Lehr, 1997 (47)).

An optical system which is linear and translation invariant, can be fully described by the image intensity distribution function of a point-shaped object, the so-called point spread function (PSF). Thus, the intensity in the image plane of an object $\mathrm{O}(\mathrm{r})$ is given as the convolution of the object function with the point spread function

$$
P(\mathbf{r})=(O * P S F)(r)
$$

In case of incoherent image formation, the intensity at the focal plane is given by equation 2.5, point spread function becomes

$$
P S F(u, v)=\left(\frac{2 J_{1}(x)}{x}\right)^{2} I_{0}
$$

The convolution in real space can be expressed as a multiplication in Fourier space

$$
\widetilde{P}\left(k_{r}\right)=\widetilde{O}\left(k_{r}\right) \cdot \widetilde{P S F}\left(k_{r}\right)=\widetilde{O}\left(k_{r}\right) \cdot O T F\left(k_{r}\right)
$$

The Fourier transform of the point spread function is called optical transfer function (OTF). The modulus of the optical transfer function is the modulation transformation function (MTF). The modulus transfer function describes the contrast transfer from the object to the image as a function of spatial frequency $\nu$ :

$$
M T F_{\nu}=\frac{I_{\max }-I_{\min }}{I_{\max }+I_{\min }}
$$

The image formation in soft X-ray microscopy is equal to the case of visible light microscopy under certain conditions, as discussed in the following. However, when transfering the schematic shown in figure 2.1 to the optical setup of an X-ray microscope, the center stop of the condenser zone plate has to be taken into account. It inhibits the direct beam to hit the sample, thus the $0^{\text {th }}$ order forms a ring instead of a circle at the backward focal plane. 

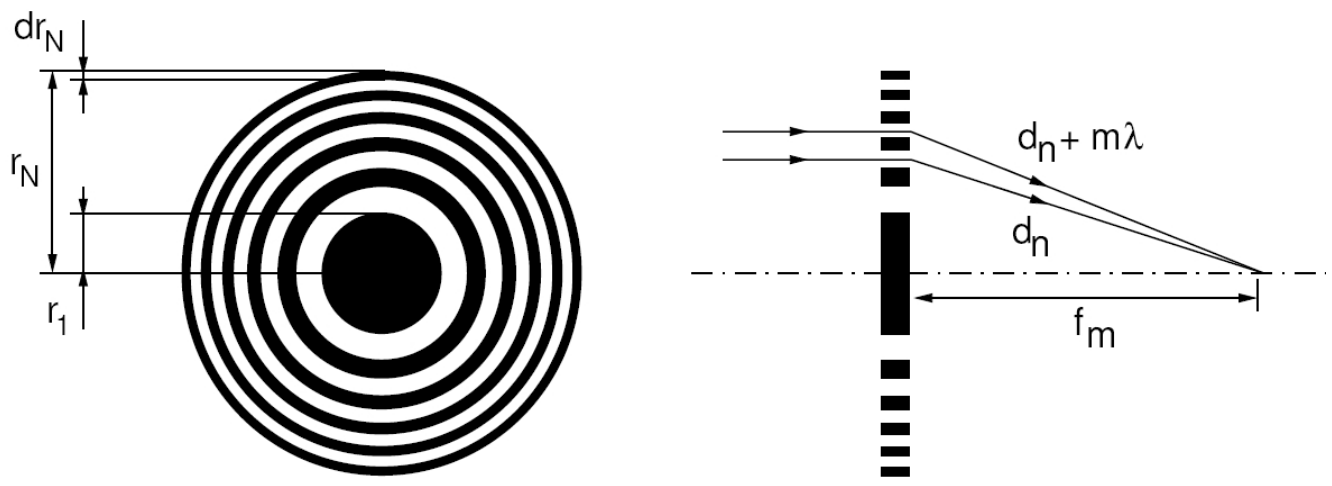

Fig. 2.2: Schematic of a Fresnel zone plate. Left: Front view. N concentric zones with radial decreasing radii $r_{n}$. The feature parameters are marked: zone plate radius $r_{N}$, radius of beam stop $r_{1}$, and outermost zone width $d r_{n}$. Right: Side view, cross section. The incident parallel light (from top left side) passes transparent zones and is focused by constructive interference in the focal spot $f_{m}$ of the $m^{\text {th }}$ order. The optical axis is depicted as dot-dashed line.

\subsection{Zone plates as soft X-ray optics}

For focusing of a soft X-ray beam, Fresnel zone plates are commonly used as diffractive optics (Schmahl et al., 1969 (64), Schmahl et al., 1984 (65), Maser, 1994 (48)). Fresnel zone plates consist in circular opaque and transparent zones, which are ordered concentrically with radially increasing zone density, thus decreasing zone width (figure $2.2)$.

A Fresnel zone plate focuses parallel incident light by constructive interference. The optical path difference has to be $m \cdot \lambda$ for adjacent transparent zones, so the passing light interferes constructively at the focal spot $f_{m}$ of the $m^{\text {th }}$ diffraction order. Therefore, the radius of the $n^{\text {th }}$ zone follows in good approximation the equation

$$
r_{n}^{2}=m n \lambda f_{m}+\frac{(m n \lambda)^{2}}{4} \quad n=1 \cdots N
$$

Differentiation with respect to $n$ where $d_{n}=1$ leads to the zone width $d r_{n}$ of the $n^{\text {th }}$ zone

$$
d r_{n}=\frac{m \lambda f_{m}}{2 r_{n}}+\frac{m^{2} n \lambda^{2}}{4 r_{n}}
$$

The second term on the right side of equation 2.12 corresponds to the correction of the spherical abberation and is negligible for large focal length $(f g g m n \lambda / 4)$. Then, the focal length for the $m^{\text {th }}$ diffraction order is given by

$$
f_{m}=\frac{2 r_{n}}{m n \lambda}
$$


Concerning the optical properties, a Fresnel zone plate with more than 100 zones can be treated as a thin lens (Michette, 1986 (51)). Thus, the point spread function as introduced in equation 2.9 describes the image formation in a soft X-ray microscope for incoherent illumination and monochromatic light. Then, the achievable lateral resolution is given by the Rayleigh criterion for monochromatic, incoherent illumination (Attwood, 1999 (5)):

$$
\delta_{l}=0.61 \frac{\lambda}{\mathrm{NA}}=1.22 \frac{d r_{n}}{m}
$$

with the numerical aperture NA given for small aperture angles $\alpha$ as

$$
\mathrm{NA}=\sin \alpha=\frac{m \lambda}{2 d r_{n}}
$$

The 1/NA dependence of the lateral resolution (equation 2.15) shows an increase of resolution when imaging with a higher diffractive order than 1 . However, the efficiency $\eta_{m}$ of an absorption zone plate decreases for higher diffraction order as (Kirz, 1974 (38))

$$
\delta_{m}=\frac{I_{m}}{I_{0}} \sim \frac{1}{m^{2}}
$$

with the initial light intensity $I_{0}$ and $I_{m}$ the intensity in the $m^{\text {th }}$ diffraction order. Due to this high loss of intensity, at present the soft X-ray microscopes work by default in the first diffractive order.

The microscopes used for this work are all based on zone plate optics (chapter 4). However, another device for focusing soft X-rays are capillaries. A recent introduction of capillary condenser optics in soft X-ray microscopy is done at the new transmission X-ray microscope at BESSY II (Heim et al., 2008 (26) and Guttmann et al., 2008 (25). Futhermore, investigations for high-resolution hard X-ray imaging are perfomed with wave guide optics (Jarre et al., 2007 (35), Ollinger et al., 2007 (59)).

\subsubsection{Depth of focus}

Especially for the investigation of spatial objects with microscopy, it is important to consider the imaging properties in depth, thus the depth of focus. There are three main definitions of the depth of focus (figure 2.3). One common definition is based on the distance from the focus along the optical axis, at which the lateral spread of focus spot equals the lateral resolution limit $\delta_{l}$ (equation 2.15). The ratio of the such defined depth of focus $\delta_{1}$ over the lateral resolution $\delta_{l}$ is given by intercept theorems as

$$
\frac{\delta_{1}}{\delta_{l}}=\frac{r_{n}}{f}=\frac{1}{\mathrm{NA}}
$$




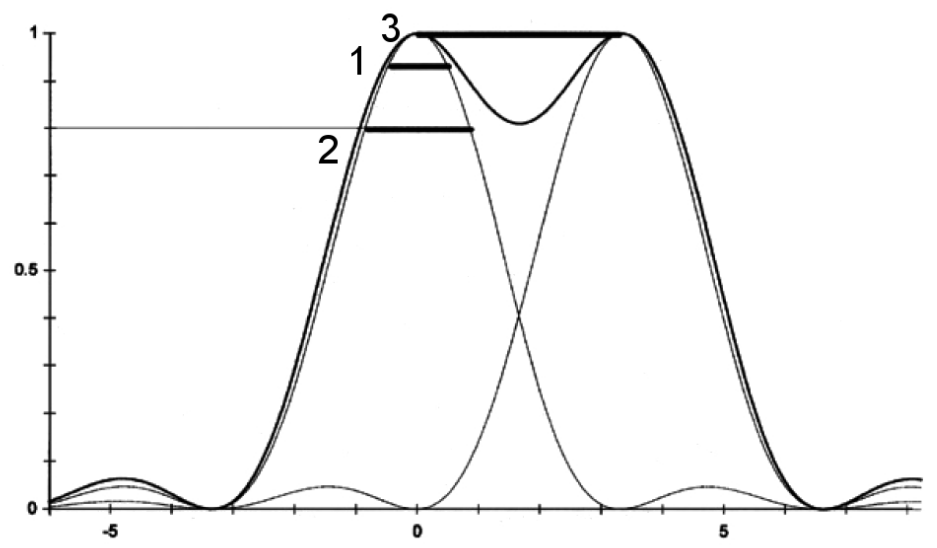

Fig. 2.3: Different definitions of the depth of focus, depicted by the intensity distributions of two point images. The definition by $\delta_{1}$ (equation 2.19) and $\delta_{2}$ (equation 2.20) refer to the lengths shown with lines 1 and 2, respectively. They describe a minimal decrease of intensity for the point image along the optical axis. The third definition, $\delta_{3}$ (equation 2.21), means the minimal distance two points may have along the optical axis, in such way that their images can still be resolved according to the Reileigh criterion (line 3).

Thus, with the definitions for $\delta_{l}$ (equation 2.15) and the numerical aperture NA (equation 2.16), the depth of focus $\delta_{1}$ results as

$$
\delta_{1}=\frac{\delta_{l}}{\mathrm{NA}}=0.61 \frac{\lambda}{\mathrm{NA}^{2}}=2.44 \frac{d r_{n}^{2}}{m^{2} \lambda}
$$

(line 1 in figure 2.3).

Another definition requires for the depth of focus a maximal decrease of intensity to $80 \%$ of the intensity at the focus. The intensity distribution along the optical axis is given in equation 2.7. It decreases by $20 \%$ for $x \approx 3 / 8$. The corresponding depth of focus $\delta_{2}$ is calculated to

$$
\delta_{2}=\frac{\lambda}{\mathrm{NA}^{2}}=4 \frac{d r_{n}^{2}}{m^{2} \lambda}
$$

(line 2 in figure 2.3).

Applying the Rayleigh criterion to the intensity distribution of an point image along the optical axis, the minimal distance $\delta_{3}$ between two points which can be still resolved, is given by (Born and Wolf, 1980 (9))

$$
\delta_{3}=2 \frac{\lambda}{\mathrm{NA}^{2}}=8 \frac{d r_{n}^{2}}{m^{2} \lambda}
$$

(line 3 in figure 2.3). 
Thus, the third criterion gives the resolution limit along the optical axis. This is of relevance for optical serial sectioning (Lehr, 1997 (47), and Knöchel, 2005 (40)). In contrast, the first two criteria give the depth of focus, determining the maximum distance along the optical axis away from the focus an object can be situated and still be imaged sharply. Thereby, the depth of focus gives a limit to the spatial extension of an object, at which all structures still appear sharply in the image. The depth of focus is depicted by the elongation of the modulation transfer function (section 2.1).

All criteria imply a ratio of depth of focus to lateral resolution much smaller than 1 , dependent on the numerical aperture of the zone plate:

$$
N A=\frac{\delta_{l}}{\delta_{t}}=\frac{\lambda}{d r_{n}}
$$

The point spread function is influenced by the monochromaticity of the illuminating light (Weiß, 2000 (94)). The monochromaticity of an initial wavelength $\lambda_{0}$ distributed by a point source is determined by the ratio of the radius $r_{n}$ of the condenser zone plate and the diameter $d$ of the pinhole:

$$
\frac{\lambda_{0}}{\Delta \lambda}=\frac{r_{n}}{d}
$$

where $\Delta \lambda$ is defined as full width at half maximum of the intensity as a function of wavelength.

The condenser zone plate of an X-ray microscope is not illuminated with finite monochromatic light, but with light of narrow-bandwidth, depending on the monochromator (chapter 4). Light with different wavelengths is focused at different focal points, each one with a particular corresponding point spread function. The addition of these point spread functions weighted with $\lambda$ forms the effective point spread function for a narrow-bandwidth illumination. A comparision of the normalised point spread function $P S F / I_{0}$ is depicted in figure 2.4 [from Weiß et al., 2000 (95)], revealing an elongation of the point spread function for a narrow-bandwidth. The horizontal image axis corresponds to the optical axis, and the vertical image axis corresponds to the distance in the $x-y$-plane from the optical axis in cylindrical coordinates, where the vertical scale is streched by a factor of 10 . The calculations of the point spread functions were done for a zone plate with an outermost zone width $d r_{n}=40 \mathrm{~nm}$ and a numerical aperture of $\mathrm{NA}=0.03$. The bottom part (a) shows the intensity distribution for monochromatic radiation with $2.4 \mathrm{~nm}$ wavelength, the top part (b) depicts the intensity distribution for a narrow-bandwidth radiation with a main wavelength of $2.4 \mathrm{~nm}$ and a monochromaticity of 200. In case of the narrow-bandwidth radiation, an elongation of the intensity distribution in $\mathrm{z}$-direction is clearly visible. This means that with incrasing monochromaticity, 


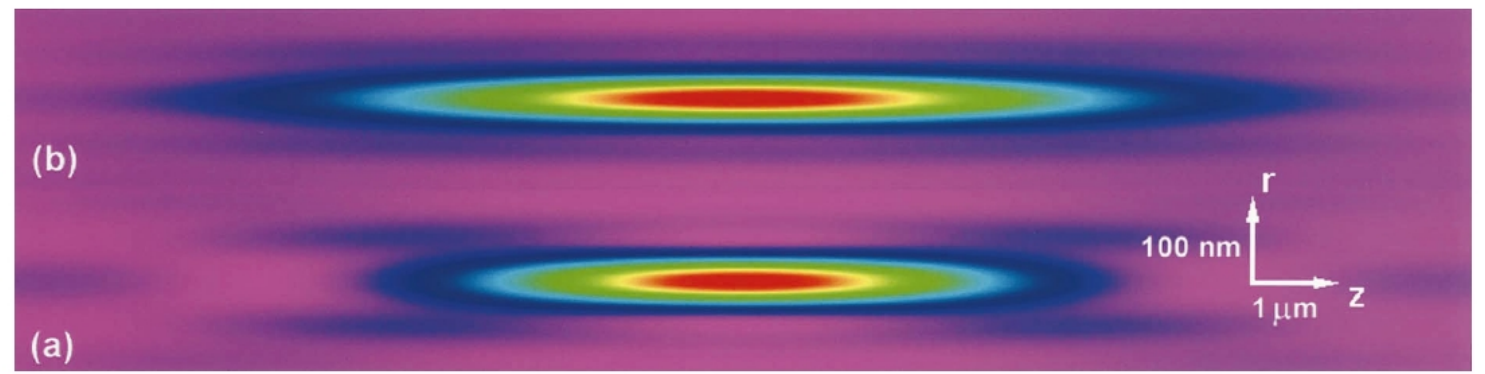

Fig. 2.4: Intensity distribution near the focal point for illumination with (a) monochromatic radiation of $\lambda=2.4 \mathrm{~nm}$ and (b) narrow-bandwidth radiation of $\lambda_{0}=2.4 \mathrm{~nm}$ and $\lambda / \Delta \lambda 200 \mathrm{~nm}$. Colour scale of normalised intensity: violet $=0$, blue $=0.25$, green $=0.6$, yellow $=0.75$, red $=1$. [From Weiß et al., 2000 (95)]

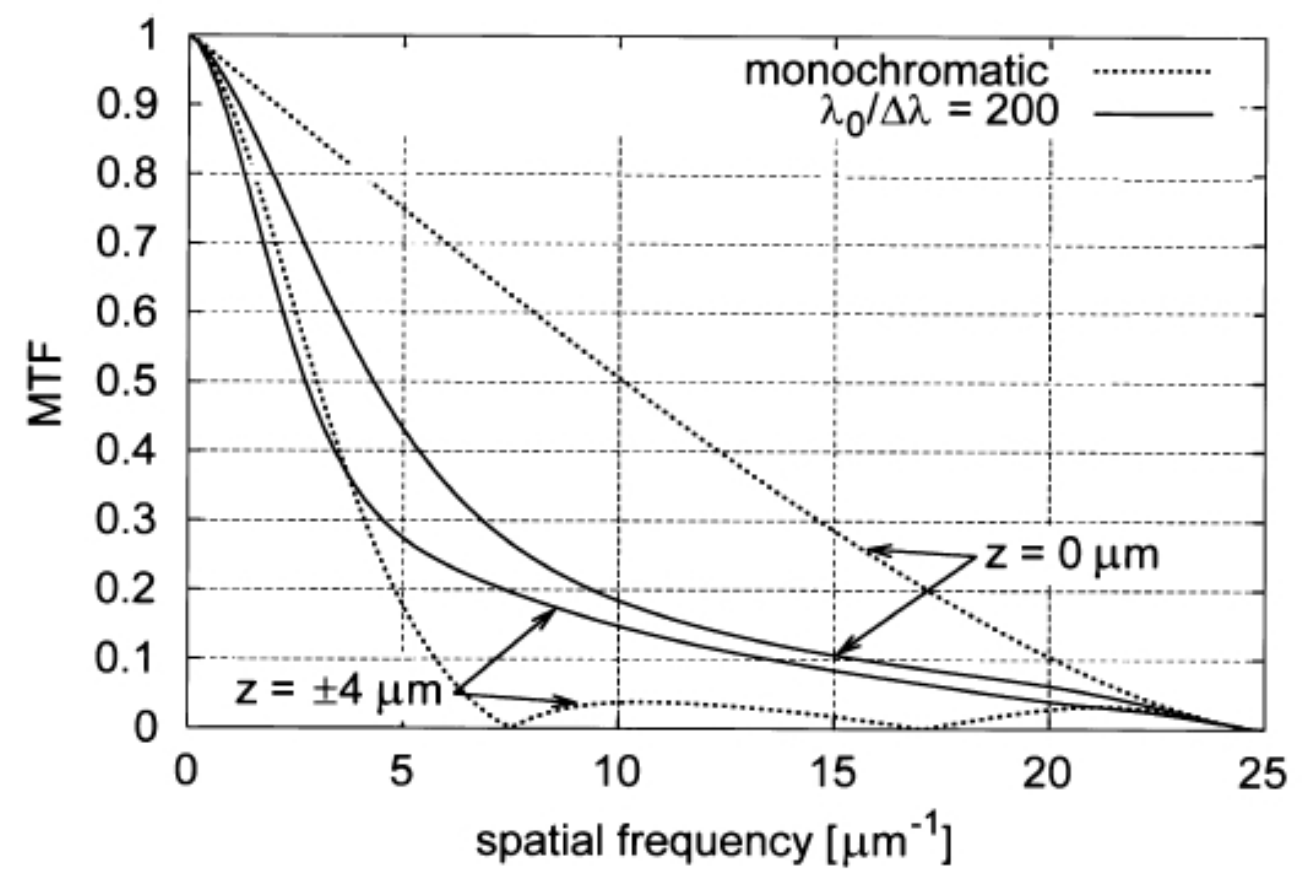

Fig. 2.5: Plot of the modulation transfer function as function of spatial frequency for both monochromatic (dashed line) and narrow bandwidth radiation (solid line) as considered for figure 2.4. The differences in resolution for an object situated in the focal plane $(z=0 \mu \mathrm{m})$ and $4 \mu \mathrm{m}$ along the optical axis out of focus are visible. While monochromatic radiation images a focused object with highest resolution, a defocused object is imaged with a very low resolution. Narrow-bandwidth radiation provides less resolution than the monochromatic radiation for the focused object, but higher resolution for the defocused object. [From Weiß et al., 2000 (95)] 
the depth of focus decreases, while the lateral resolution increases. The relevance when imaging an extended object is demonstrated in figure 2.5[from Weiß et al., 2000 (95)]. The modulation transfer function is plotted as a function of spatial frequency for both monochromatic (dashed line) and narrow bandwidth radiation (solid line) as considered for figure 2.4, where the cut-off frequency for Rayleigh resolution corresponds to $9 \%$ of the modulation transfer function. The differences in resolution for an object situated in the focal plane $(\mathrm{z}=0 \mu \mathrm{m})$ and at $4 \mu \mathrm{m}$ along the optical axis out of focus are visible. While monochromatic radiation images a focused object with highest resolution, a defocused object is imaged with a very low resolution. In contrast, narrow-bandwidth radiation provides less resolution than the monochromatic radiation for the focused object, but higher resolution for the defocused object. An extended object means that object structures away from the focal spot have to be imaged. In the given example, a position of $4 \mu \mathrm{m}$ out of focus is considered, corresponding to a spatial object of $8 \mu \mathrm{m}$ extension in z-direction. Imaging such extended objects with highly monochromatic radiation means a loss of information for structures out of focus. In this case, images at different focii have to be taken to reveal structures with distances in z-direction (method of serial sectioning explained for example by Lehr, 1997 (47), and Knöchel, 2005 (40)).

Due to the refractive index and the relatively large depth of focus, depicted in the elongation of the point spread function in figure 2.4, the assumption is made that soft Xray microscopy imaging can be treated as a parallel projection. On this assumption, both tomographic reconstruction (Lehr, 1997 (47) and Weiss, 2000 (94)) and stereo processing (chapter 3) are based. However, this assumption is not true anymore for zone plates with higher numerical aperture. In that case, image formation is more complex. An extensive description of the optical transfer function at several relevant configurations of the optical setup of an X-ray microscope, also considering zone plates with higher numerical aperture, can be found in Knöchel, 2005 (40). In order to compensate for the decreased depth of focus, Knöchel made first experiments combining optical serial sectioning and tomography, but the reconstruction has not proved satisfactory.

\subsection{Absorption contrast}

The interaction of soft $\mathrm{X}$-rays in the range of about $0.1 \mathrm{keV}$ to $2 \mathrm{keV}$ with matter is dominated by photoelectric absorption. The cross-section for elastic scattering is notably 
smaller, while the cross-section for inelastic scattering is negligible. This interaction is described at atomic scale by the atomic scattering factor for forward scattering:

$$
f=f_{1}+i f_{2}
$$

In Henke et al., 1993 (27), the values of $f_{1}$ and $f_{2}$ for nearly all materials are listed based on experimental results. (An extended and corrected version of the Henke tables is available online at http://henke.lbl.gov/optical_constants.)

When passing an extended object, the electromagnetic wave experiences multiple interaction with atoms. This leads to a superposition of the incident wave with the waves scattered in direction of propagation. Thereby, the incident electromagnetic wave is attenuated and phase shifted. Both effects are described by the refractive index

$$
n=1-\delta-i \beta=1-\frac{\rho_{\mathrm{a}} \mathrm{r}_{\mathrm{e}} \lambda^{2}}{2 \pi}\left(f_{1}+i f_{2}\right)
$$

with the atomic density $\rho_{a}$, the classical electron radius $\mathrm{r}_{e}$, and wavelength $\lambda$. It is notable that for soft $\mathrm{X}$-rays the refractive index is smaller than 1 , and $\beta$ and $\delta$ are in the range of $10^{-2}$ to $10^{-6}$.

A plane wave propagating in vacuum can be described as

$$
\psi(z)=\psi_{0} \mathrm{e}^{i(\omega t-k z)}
$$

with propagation direction $z$, amplitude $\psi_{0}$, circular frequency $\omega$, and wavenumber $k=2 \pi / \lambda$. When propagating in a homogeneous material, the wave can be described as

$$
\psi(z)=\psi_{0} \mathrm{e}^{i(\omega t-k z)} \mathrm{e}^{i \delta k z} \mathrm{e}^{-\beta k z}
$$

Thus, the absorption is given by $\beta$ and the phase shift by $\delta$.

However, the detectable value of the electromagnetic wave is the intensity $I$. The material specific decrease of the intensity is given by the Lambert-Beer law:

$$
\frac{I}{I_{0}}=\mathrm{e}^{-\mu d}
$$

with the linear absorption coefficient $\mu$

$$
\mu=\frac{4 \pi \beta}{\lambda}
$$

The absorption length $\mu^{-1}$ determines the thickness of the material, which reduces a transmitted electromagnetic wave by a factor of e. The mass attenuation coefficient $\mu_{m}$ 


\begin{tabular}{ccccc}
\hline & $280 \mathrm{eV}$ & $360 \mathrm{eV}$ & $400 \mathrm{eV}$ & $700 \mathrm{eV}$ \\
\hline $\mathrm{Si}_{3} \mathrm{~N}_{4}+2 \mu \mathrm{m}$ water & 0.067 & 0.22 & 0.31 & 0.087 \\
$\mathrm{Si}_{3} \mathrm{~N}_{4}+6 \mu \mathrm{m}$ water & 0.008 & 0.071 & 0.131 & 0.001 \\
$2 \mu \mathrm{m}$ capillary $+1.6 \mu \mathrm{m}$ water & 0.082 & 0.189 & 0.278 & 0.082 \\
$6 \mu \mathrm{m}$ capillary $+4.8 \mu \mathrm{m}$ water & 0.00008 & 0.007 & 0.022 & 0.0001 \\
\hline
\end{tabular}

Table 2.1: X-ray transmission at various energies of different glass capillaries and stacks of $\mathrm{Si}_{3} \mathrm{~N}_{4}$ membranes filled with water.

is defined as the ratio of the linear absorption coefficient over the mass density of the material $\rho$ :

$$
\mu_{m}=\frac{\mu}{\rho}
$$

The energy dependence of the absorption coefficient (equation 2.29) is discussed in chapter 2.4.

The resulting X-ray transmission for both kinds of water filled sample holders used in the experiments and described in chapter 3.1 is shown for some relevant energies in table 2.3. The capillaries consist of borosilicate, which has a chemical composition of about $56 \% \mathrm{SiO}_{2}, 18 \% \mathrm{Na}_{2} \mathrm{O}, 14 \% \mathrm{~B}_{2} \mathrm{O}_{3}, 4 \% \mathrm{TiO}_{2}, 4 \% \mathrm{CaO}, 2 \% \mathrm{MgO}$, and $2 \%$ $\mathrm{Al}_{2} \mathrm{O}_{3}$, and the density is $2,77 \mathrm{~g}$ per $\mathrm{cm}^{3}$. They have been considered with $2 \mu \mathrm{m}$ and $6 \mu \mathrm{m}$ thickness. The wall thickness of the capillaries corresponds to about $10 \%$ of the capillary diameter. Thus, for the $1 / e$ transmission of a capillary of $2 \mu \mathrm{m}$ diameter, a borosilicate thickness of $0.4 \mu \mathrm{m}$ and a water thickness of $1.6 \mu \mathrm{m}$ are considered. The stack of $\mathrm{Si}_{3} \mathrm{~N}_{4}$ foils consists of two $\mathrm{Si}_{3} \mathrm{~N}_{4}$ foils with $100 \mathrm{~nm}$ thickness each. Water layers between the $\mathrm{Si}_{3} \mathrm{~N}_{4}$ foils of $2 \mu \mathrm{m}$ and $6 \mu \mathrm{m}$ are surveyed. The chosen energies correspond to the energies used at the particular X-ray microscopy stations (chapter 4).

\subsubsection{Radiation damage}

As mentioned in chapter 2.3, optical properties of matter are a result of the material's electron configuration, dominated in the soft X-ray range by photoelectric absorption and elastic scattering. The absorption of X-radiation in the sample leads to radiation damage, mostly due to released secondary electrons. However, a minimum dosage has to be applied to obtain a high resolution X-ray micrograph with a reasonable signal-tonoise ratio. Calculations of the optimum imaging photon density and dosage with regard 
to wavelength, resolution, and different contrast modes have been studied in detail by Schneider (Schneider, 1992 (66) and Schneider, 1999 (67)).

For radiation sensive matter like proteins, cryo fixation makes it possible to apply high dosages of $10^{9}$ to $10^{10} \mathrm{~Gy}$, impeding structural changes in the size range above the resolution limit of an X-ray microscope. At higher dosages, radiolysis starts. With cryo fixation, introduced to soft X-ray microscopy by Schneider, 1999(67), even tomographic data sets can be recorded from biological samples (Schneider et al., 2002 (68)).

The method of cryo fixation is also employed when taking tomographic data sets of less radiation sensitive samples for the reason of preventing sample movement during the time lag necessary for recording more than $60 \mathrm{X}$-ray micrographs as necessary for a tomographic data set. The requirements for X-ray tomography can be found in Knöchel, 2005 (40). X-ray tomography of not radiation sensitive samples is presented in Knöchel et al., 2003 (41) and Thieme et al., 2003 (88). Cryo fixation is also applied in soft X-ray spectromicroscopy (chapter 2.4) for the combination of spectromicroscopy and tomography (Maser et al., 1999 (91) and Wang et al., 1999 (92)).

\subsection{Spectromicroscopy}

Taking advantage of the element specific, energy dependent absorption of X-radiation (chapter 2.4.1), spectromicroscopy combines the elemental and chemical sensitivity with high spatial resolution. Such experiments require highly monochromatic radiation for high resolution, commonly provided at scanning X-ray microscopes (STXM, chapter 4.2.1). At the same time, for high spatial resolution, the X-radiation has to be focused to a small spot and then scanned with an accuracy in the nanometre range over the sample. A spectrum for each sample point is obtained by scanning the sample subsequently with different energies in small energy steps around an absorption edge, thus forming a stack of images taken at different energies. By spectral analysis, chemical information is then available for each point of the sample and can be correlated with particular regions of inhomogenious samples.

\subsubsection{Absorption spectra}

Figure 2.6 shows a schematic of the electron transition by the absorption of a photon from the electron potential of a diatomic molecule (left) and the corresponding K-shell absorption spectrum (right). The spectrum shows the resonance peaks for the transition of the excited electron to unoccupied levels and the absorption edge matching with the 


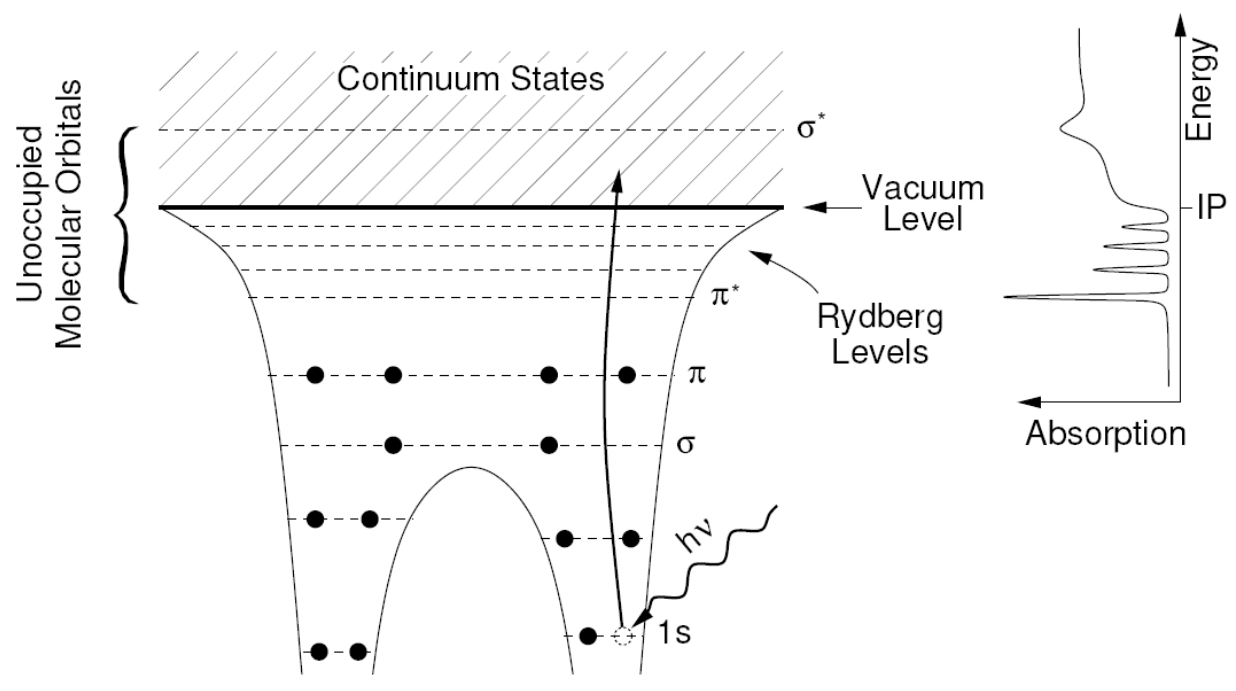

Fig. 2.6: Schematic of electron transition by photon absorption from the electron potential of a diatomic molecule (left) and corresponding K-shell absorption spectrum (right). The spectrum shows the resonance peaks for the transition of the electron to unoccupied levels and the absorption edge matching with the ionisation potential IP. [From Wiesemann, $2003(96)]$

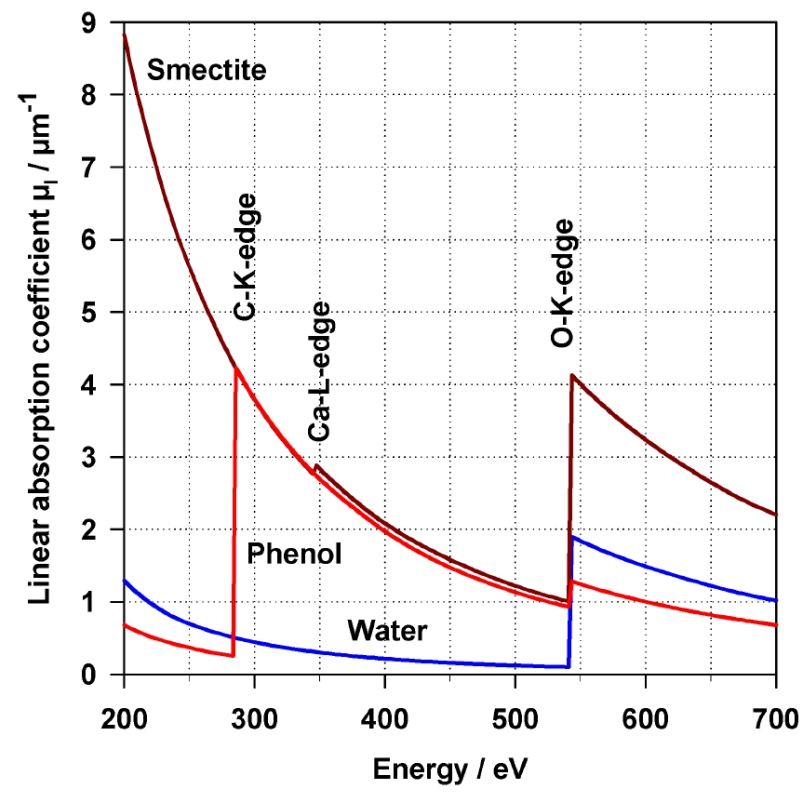

Fig. 2.7: Linear absorption coefficients of water, phenol, and smectite as a function of energy. Due to the much weaker $X$-ray absorption of water compared to smectite and phenol (above the $K$ absorption edge of $C$ ), a natural absorption contrast exists and smectite and phenol can be imaged in aqueous media of several micrometre thickness. [From Mitrea et al., 2008 $(52)]$ 


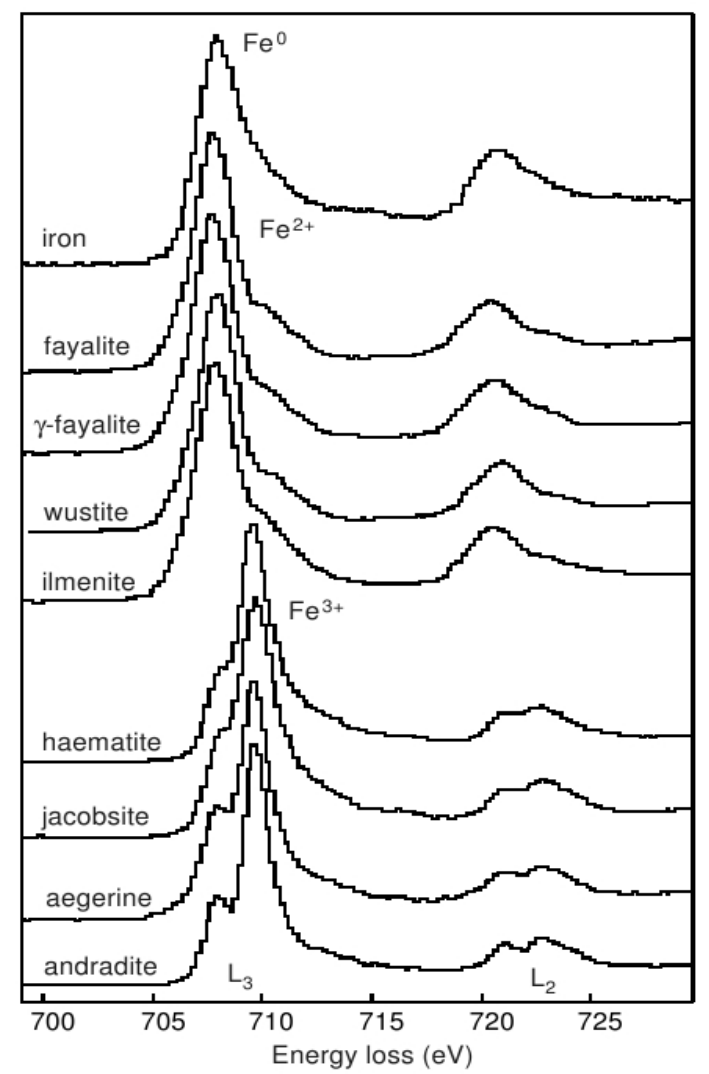

Fig. 2.8: Spectra from elemental iron and single valent iron minerals around L3 and L2 absorption edges of iron. Shifts in peak positions are visible. [From Garvie and Buseck, 1998 (19)]

ionisation potential IP. At energies below the absorption edge, excitations to unoccupied molecular orbitals lead to strong absorption peaks in the absorption spectrum. At energies above the absorption energy, an electron is emitted. Backscattering waves of such emitted electron by the neighboring atoms lead to interference, depending on the de Brogglie wavelength either constructive or destructive. This interference causes fine oscillations in the absorption spectrum above the absorption edge. Including these fine oscillations occuring in an energy range of up to about $40 \mathrm{eV}$ above the absorption edge energy, the spectrum is called Near Edge Absorption Fine Spectructure (NEXAFS) spectrum (Stöhr, 1996 (78), Ishii and Hitchcock, 1987 (31)). Thus, the element specific absorption coefficient $\mu$ (equation 2.29) depends on the radiation energy, as shown in figure 2.7. The linear absorption coefficients of water, phenol, an organic molecule, and smectite, a 2:1 clay mineral (chapter 5.1) are plotted as a function of energy in the range of $200 \mathrm{eV}$ to $700 \mathrm{eV}$ [from Mitrea et al., 2008 (52)]. Three absorption edges included in this energy range are depicted (chapter 2.4.2). Due to the much weaker X-ray ab- 
sorption of water compared with smectite and phenol (above the $\mathrm{K}$ absorption edge of C), a natural absorption contrast exists and smectite and phenol can be imaged under aqueous conditions with a water layer of several micrometre thickness (confirm table $2.3)$.

The capability of NEXAFS is demonstrated in figure 2.8, showing spectra from elemental iron and various single valent iron minerals taken around the L 3 and L 2 absorption edges of iron [from Garvie and Buseck, 1998 (19)]. The slight differences in the particular NEXAFS spectra and the visible shifts in peak positions provide the possibility to distinguish between the iron minerals.

\subsubsection{Elemental mapping}

However, for many questions it is sufficient to reveal the elemental distribution within an inhomogeneous sample. Due to the the element and shell specific X-ray absorption edges (figure 2.7), it is possible to take two X-ray micrographs of the same sample region but one taken at an energy slightly below a specific absorption edge, and one above. A qualitative distribution map of the specific element is then created by dividing the image taken above the absorption edge energy by the one taken below the absorption edge energy:

$$
I_{\text {map }}=\ln \left(\frac{I_{\text {above }}}{I_{\text {below }}}\right)=\ln \left(\frac{I_{0} e^{-\mu_{a} d}}{I_{0} e^{-\mu_{b} d}}\right)
$$

Examples for such elemental distribution maps can be found in chapter 6, for example the distribution of the iron oxide haematite within a clay sample is presented in figure 6.17 .

Furthermore, based on the Lambert-Beer law (equation 2.28) and assuming an $1 / E^{3}$ dependence for the mass attenuation coefficient, a map of thickness of the respective element can be created by

$$
d_{e l}=\frac{\ln \left(I_{02} / I_{2}\right)-\left(E_{1} / E_{2}\right)^{3} \ln \left(I_{01} / I_{1}\right)}{\mu_{l 2}-\mu_{l 1}\left(E_{1} / E_{2}\right)^{3}}
$$

(Neuhäussler, $1999(55)$ ), where $I_{01}$ and $I_{02}$ are the intensities of the incident X-ray beams at the respective energies $E_{1}$ and $E_{2}$, while $I_{1}$ and $I_{2}$ are the intensities of the transmitted X-radiation at $E_{1}$ and $E_{2}$, respectively. The linear absorption coefficients $\mu_{l 1}\left(\right.$ at $\left.E_{1}\right)$ and $\mu_{l 2}$ (at $E_{2}$ ) are known from tabulated data (Henke et al., 1993 (27)). An example for such a mass thickness map is given for the carbon content of a chernozem sample by Mitrea et al., 2008 (52).

The calculation of thickness maps of an element with respect to its density, and an application of this technique can be found in Buckley et al., 1998 (11). 


\section{Chapter 3}

\section{Stereo microscopy}

In many scientific problems it is important to know the spatial distribution within a sample under investigation. This explains the enormous success of tomography, e.g. computer tomography with hard X-rays, magnetic resonance tomography, electron tomography, ultrasonic tomography, positron emission tomography and single photon emission tomography, with a wide range of applications in medicine, biology, environmental sciences and geology among others. Tomography (Greek: tomos $=$ slice) is a method to reconstruct slices of an object from many projection images taken under different angles over a tilt range of preferably 180 degree. The reconstruction based on the Radon transformation (Radon, 1917 (62)) enables the reconstruction of slices through an object (Natterer, 1986 (54)). The ensemble of these slices forms a spatial reconstruction of the object and its inner structures. In soft X-ray microscopy, the three-dimensional reconstruction of the sample is gained as well from a set of projections formed by at least 60 two-dimensional transmission images taken over a tilt range of 180 degree (Lehr, 1997 (47)). Thereby, the sample is exposed to a high dosage and has to be cryo fixated to avoid visible radiation damage (Schneider, 1999 (67)).

While tomography provides a complete reconstruction of an object, some spatial information can also be gained by just two tilted projection images forming a stereo image pair. Both imaging and processing effort are much less compared to tomography, and the restricted spatial information gained can be sufficient, depending on the scientific problem addressed. In this work, a software package called xstereo has been written for analysing stereo micrographs (section 3.3). Significant structures are marked manually in a stereo pair of micrographs and information about the three-dimensional configuration, distances and lengths is obtained. In a three-dimensional plot, features are displayed and formations like curvatures are depicted. 


\subsection{Sample holders}

As tiltable holders for experiments with aqueous samples thin-pulled glass capillaries can be used, which are already common for X-ray tomography (Lehr, 1997 (47), Schneider et al., 2002 (68), Larabell et al., (46)). For these studies, borosilicate glass capillaries (fabricated by Hilgenberg GmbH, Germany) starting with $1 \mathrm{~mm}$ outer diameter and $0.1 \mathrm{~mm}$ wall thickness were used. For easier filling, the capillaries have a $0.1 \mathrm{~mm}$ thick glass filament attached to the inner wall, improving the capillary forces when filled with a liquid. Capillary tips down to diameters of $1 \mu \mathrm{m}$ and lengths short enough that the tips stay stable without movements in the microscope throughout the experiment have been manufactured with an in-house pipette puller (fabricated by H. Saur Laborbedarf, Germany). The initial capillary is mounted horizontally to the pipette puller, passing through a circular heating filament, and fixed on both ends with clamps onto sliding carriages. Both carriages are driven by variable pulling forces. When those get unlocked, a symmetric pulling force is exerted to the glass capillary. The heating filament heats the enclosed central part of the capillary. Close to the melting point, the glass becomes so soft that it cannot stand the pulling force of the carriages and both ends of the capillary get drawn apart. Thus, two capillaries with thin tips are obtained. By regulating the pulling forces as well as the heating current, angle, diameter and length of the taper are controlled. For the experiments presented here, the tip diameters could have been adjusted between one and eight micrometres and the lengths were around five millimetres to optimise between observable sample volume and tip stiffness.

For experiments, the capillary is filled from the wide end inserting a microloader pipette tip (Eppendorf AG, Germany) with a volume of approximately $10 \mu$ l containing the aqueous sample. The sample is injected slowly and directly in front of the tapered region, entering the capillary tip due to capillary forces. Depending on the chemical conditions of the sample, the filling behaviour changes and the tapering angle has to be adapted. To prevent a drying-out of the filled capillary tip, its very end is sealed by dipping it under light microscopic observation into a drop of viscous glue. For manipulating the sample during the experiment, further solutions or dispersions are inserted into the capillary as before. In case of solutions, the formerly observed sample position is reached by the particles in the added solution through the concentration gradient and diffusion, whereas in case of dispersions, the observed sample position is reached through Brownian motion and diffusion. Due to the small volume, for the investigated liquid samples the mixing took place within a few minutes. For this kind of 

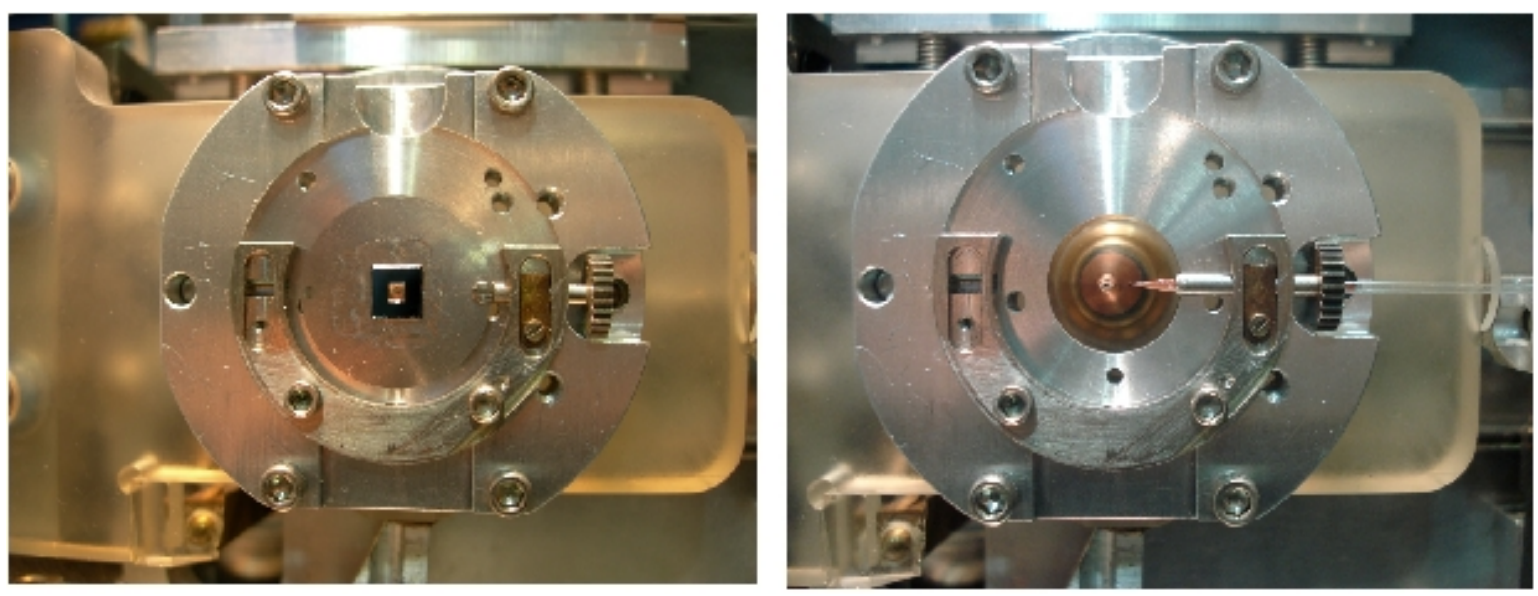

Fig. 3.1: Tiltable object holders mounted to the object stage of the X-ray microscope XM-1 at the Advanced Light Source, Berkeley. The object stage is in air, the pinhole serving as vacuum window is located on a cone behind the object holder. Left: Flat object holder consisting of a pair of $\mathrm{Si}_{3} \mathrm{~N}_{4}$ membranes glued onto an aluminium shim. The shim is fixed to the detachable tilt stage, consisting in a steel half ring and an axle tiltable via a cog wheel. The axle is clamped by a flat copper spring. Right: Thin-pulled glass capillary filled with a haematite suspension. The capillary is protruding from both sides of the steel axle, which is mounted to the detachable tilt stage.

manipulation, it is important to avoid any air bubbles in the capillary which interrupt the path for matter exchange towards the observable thin region of the tip. With regards to this, a slow filling and a small tapering angle proved to be advantageous.

To mount the capillary to the object stages of the X-ray microscopes at BESSY (chapter 4.2.1) and the ALS (chapter 4.1.1), a detachable tilt stage, introduced by Weiß, 2000 (94), has been used. The filled capillary is inserted with the thick end first into a hollow steel axle of $1 \mathrm{~mm}$ inner diameter and a cog wheel attached to one end of it (figure 3.1 right). The capillary is passed so far, that the fragile tip is enwrapped by the axle, while the back end of the capillary juts out of the $\operatorname{cog}$ wheel. In this arrangement, the axle and with it the capillary are inserted into a steel yoke. The axle is fixed with a flat spring at a corresponding cut-out. The capillary tip is pushed forward out of the axle until it reaches the centre of the yoke, which corresponds in the mounted state roughly to the position of the image field of the X-ray microscope. To secure this position from further pushing, the capillary is fixed with a drop of glue to the axle. The such compounded tilt stage is mounted to the sample stage of the microscope. A cog wheel allows for rotation of the capillary along an axis perpendicular to the optical axis either manually or using a motor. 
The tilt range is limited for an extended sample holder, because of a small airgap between the two vacuum windows where the sample is positionend, and due to the size of the pinhole mountings (chapter 4). In addition, the thickness of a flat sample layer increases when tilted and exceeds at certain point the 1/e absorption length (chapter $2.3)$.

However, extended sample holders form an advantageous setup for studying extended objects. Small tilting angles are sufficient for stereo imaging, so it is possible to place an aqueous sample into an X-ray microscope in a tiltable flat holder (figure 3.1 left). For this purpose, two $\mathrm{Si}_{3} \mathrm{~N}_{4}$ membranes (Silson Ltd, UK) of $100 \mathrm{~nm}$ thickness each have been used (membrane size: $1.5 \mathrm{~mm} \times 1.5 \mathrm{~mm}$, frame size: $5.0 \mathrm{~mm} \times 5.0 \mathrm{~mm}$, frame thickness: $200 \mu \mathrm{m})$. The dimensions of the $\mathrm{Si}_{3} \mathrm{~N}_{4}$ membranes are comparable to those of the $\mathrm{Si}_{3} \mathrm{~N}_{4}$ membranes acting as vacuum windows, thus limiting the usable tilt range. With the sample in between, a pair of $\mathrm{Si}_{3} \mathrm{~N}_{4}$ membranes is glued onto a $100 \mu \mathrm{m}$ thin aluminium shim, just across the $1 \mathrm{~mm}$ central hole of the aluminium shim. Then, the shim is mounted to the same tilt stage in a similar way as the glass capillary. Instead of the hollow steel axle, an axle with a flatened end is used, onto which the aluminium shim is tightened with a screw. The maximum tilt range between the two vacuum windows of the XM-1 is approximately 14 degrees. This is sufficient to obtain images for stereo analysis.

For the studies of extended objects, a drop of the aqueous sample is placed onto one of the $\mathrm{Si}_{3} \mathrm{~N}_{4}$ membranes and then covered with the other one. Due to capillary forces, the water layer between the two membranes adjusts to the micrometre range. Using a pair of $\mathrm{Si}_{3} \mathrm{~N}_{4}$ membranes enclosing a water layer of $2 \mu \mathrm{m}$ thickness yields a calculated X-ray transmission at $\mathrm{E}=700 \mathrm{eV}$ of $8.7 \%$ (table 2.3). To prevent the sample from drying, the pair of membranes is sealed with glue around the edges of the wafers and subsequently fixed in the same way onto the aluminium shim.

To enable manipulation of samples within such sample holder, $\mathrm{Si}_{3} \mathrm{~N}_{4}$ membranes have been designed with three channels forming a Y structure (figure 3.2 left). Two channels have a width of about $200 \mu \mathrm{m}$ for the feed of two suspensions, while the third channel with a width of about $400 \mu \mathrm{m}$ forms the drain. The centre of the Y structure is situated in the centre of the carrier $\mathrm{Si}_{3} \mathrm{~N}_{4}$ membrane to match with the object field of the $\mathrm{X}$-ray microscope when mounted. The structured $\mathrm{Si}_{3} \mathrm{~N}_{4}$ membranes are also produced by Silson, using photoresist and photolithography for creating a structure height of $3 \mu \mathrm{m}$ on top of the $\mathrm{Si}_{3} \mathrm{~N}_{4}$ membrane (figure 3.2 right). To build a fluidic cell sample holder, the structured membrane is glued with its frame onto a aluminium shim as described above 

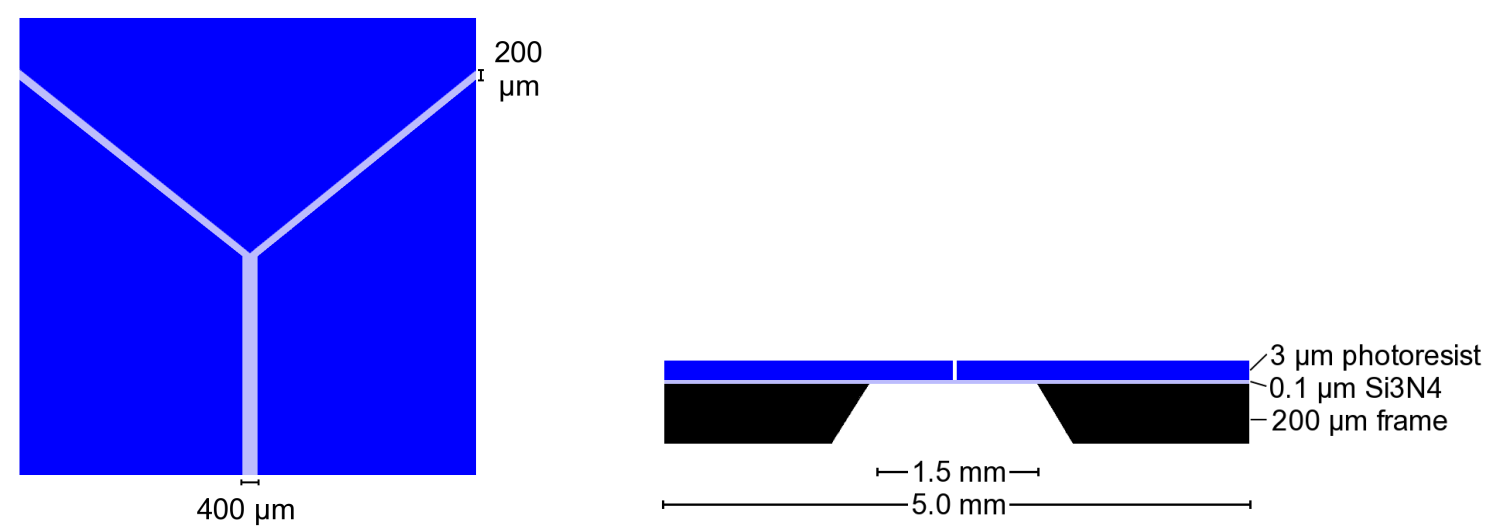

Fig. 3.2: Schematic of a $\mathrm{Si}_{3} \mathrm{~N}_{4}$ membrane coated with a $3 \mu \mathrm{m}$ layer of photoresist including channels forming a $Y$ structure to use for the manipulative fluidic cell sample holder (figure 3.3). Front view (left) and side view (right).

(figure 3.3). The glue has to control the suspension flow afterwards and is applied in form of a hollow sun with three rays: it is masking the entire centre hole to prevent leaking and three outward radial lines as barriers running parallel to the resist structures of the structured membrane. A blank $\mathrm{Si}_{3} \mathrm{~N}_{4}$ membrane is sandwiched with the membrane side downwards onto the structured one and secured with glue along the previous radial glue lines protruding the $\mathrm{Si}_{3} \mathrm{~N}_{4}$ frames by several millimetres. To access the feed channels, two thin hollow wires are fixed with drops of glue onto the aluminium shim, each one ending close to one feed channel. Thus, the sample suspensions injected to the hollow wires reach the stack of membranes and flow into the channels due to capillary forces. The suspensions coalesce in the centre of the $\mathrm{Y}$ formed channel system and flow out through the drain. As the total flow volume is just in the range of microlitres, the outflow forms at most a drop on the aluminium shim.

The fluidic cell sample holder is fixed onto the tilt stage as described above and then mounted to the X-ray microscope. The injection is performed in the mounted status, so the instantaneous reactions of two suspensions mixed can be investigated, i.e. precipitation, delamination or aggregation of the particles.

This is a unique application field compared to the experiments possible with a tapering capillary. Here, either the dynamical behaviour within one suspension like particle growth, or the mixture of two suspensions by slow diffusion can be investigated. However, the use of the fluidic cell requires on the part of the microscope more space in the object area and on the part of the samples particles big enough to get stuck between the $\mathrm{Si}_{3} \mathrm{~N}_{4}$ membranes, so they can be imaged sharply. 

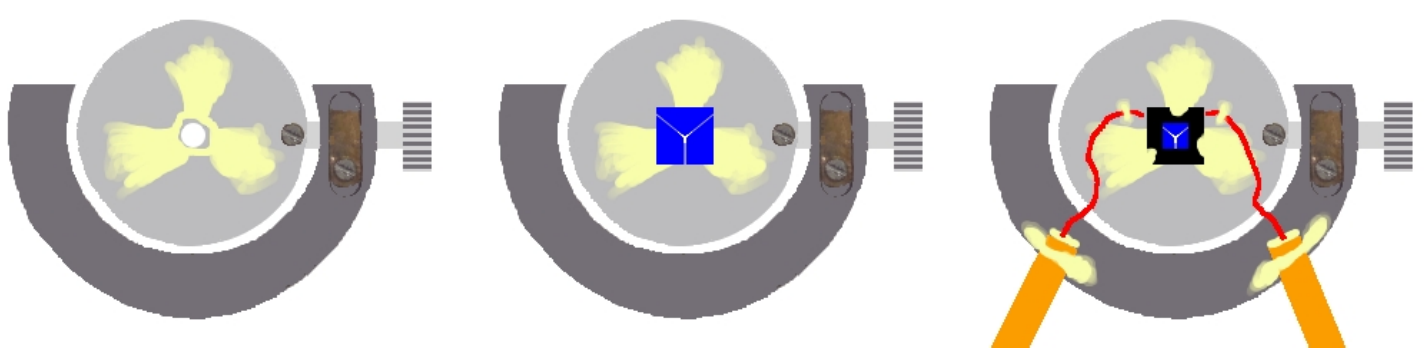

Fig. 3.3: Fluidic cell sample holder, shown in three preparation steps. Left: The aluminium shim is fixed to the detachable tilt stage. Glue is applied around the centre hole and in three rays away from it. Middle: A structured $S_{3} N_{4}$ membrane is aligned to the centre with the frame side down and the coating up. Each channel leads to one segment defined by the glue. Right: $A \mathrm{Si}_{3} \mathrm{~N}_{4}$ membrane is put with the frame side up onto the coated $\mathrm{Si}_{3} \mathrm{~N}_{4}$ membrane forming a fluidic cell. To access the feed channels, very thin copper wires are fixed close to them. The copper wires are filled via silicone hoses, which are fixed onto the steel half ring of the detachable tilt stage.

\subsection{Stereo reconstruction}

When an object is observed from two different places, it appears to be shifted in its position relatively to the background. This seeming shift is called parallax (Greek: change, discrepancy). This effect forms the basis for stereoscopy (Greek: stereo=spatial, skopein $=$ to see), and does so for binocular vision: A human pair of eyes consists of two eyes with an average distance of $63.5 \mathrm{~mm}$. Thus, each eye has its own perspective, and its view of an object is different to the other eye's view, especially when the object is close to the pair of eyes. Besides other distance depending cognitive parameters like for example sharpness, eye focus, angle between lines of sight, or size scale based on experience, the parallax gives important information for spatial perception. This information is mathematically described by the parallax equation (section 3.2.1) and applied to the parallax induced disparity in X-ray stereo micrographs (figure 3.4).

\subsubsection{Spatial coordinates calculated from parallax equation}

Stereo calculations are based on the parallax equation (equation 3.1) (20). It relates the parallax $\Delta Y$ to the vertical distance $h$ and the tilt angle $\theta=2 \theta_{\mathrm{s}}\left(\theta_{\mathrm{s}}\right.$ is the stereo angle).

In two dimensions, when tilting an L-like structure around the z-axis vertical to the object plane as can be seen in figure 3.5, the coordinates of three points a,b and 

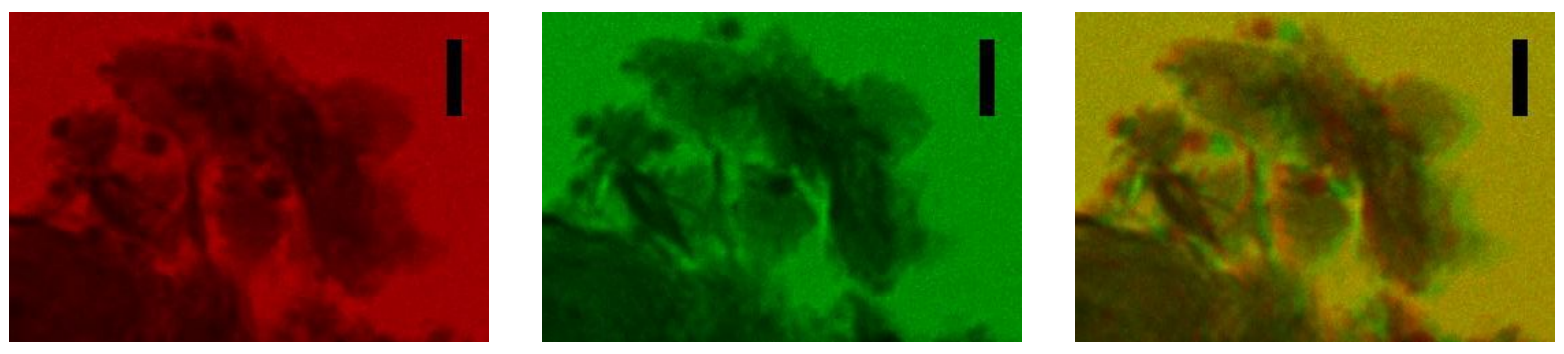

Fig. 3.4: Left and middle: Pair of stereo micrographs of an aqueous montmorillonite sample with haematite particles added (confirm section 6.5, originally same images as presented in figure 6.18) taken at $E=707 \mathrm{eV}$ with a tilt angle of $\Delta \theta=14^{\circ}$, here presented as a horizontal tilt and the micrographs displayed in red and green to enable stereo vision with Red-greenglasses. The scale bars indicate $20 \mu \mathrm{m}$. Right: Anaglyph image from the sum of left and middle image. The shift in colours clearly demonstrates the parallax induced disparity in both stereo micrographs.

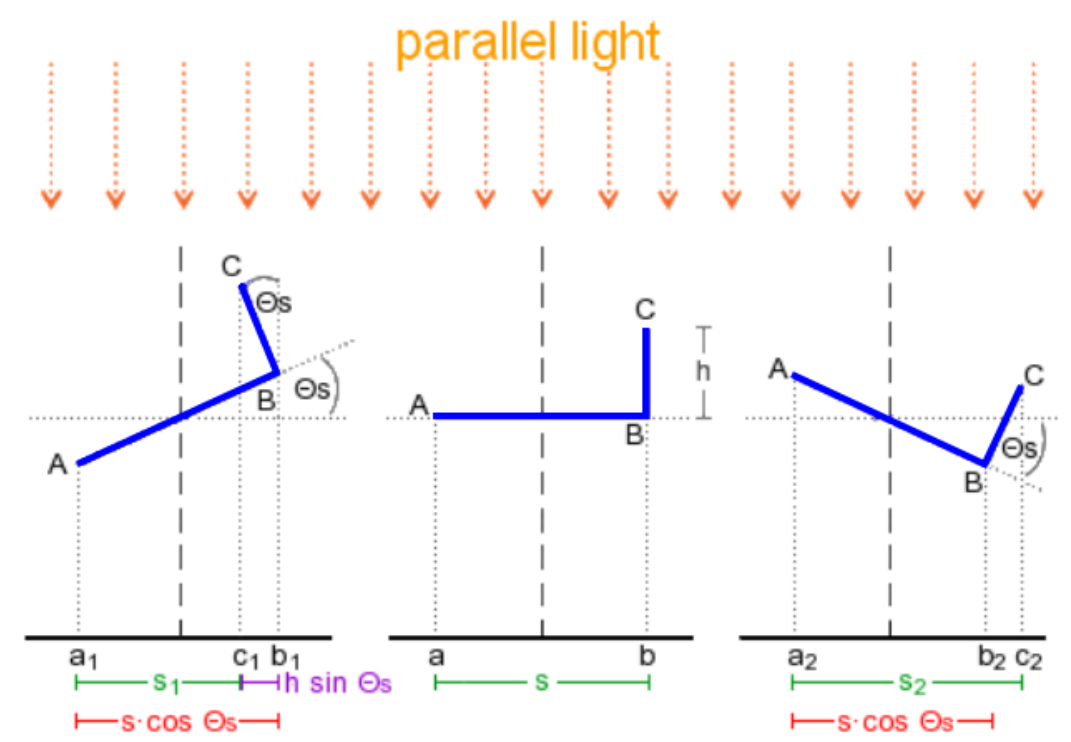

Fig. 3.5: Tilt of a two-dimensional object around the stereo angle $\theta_{\mathrm{s}}$ projected parallel onto the base line. The plotted lengths are used to derive the parallax equation (equation 3.1, please confirm text). 


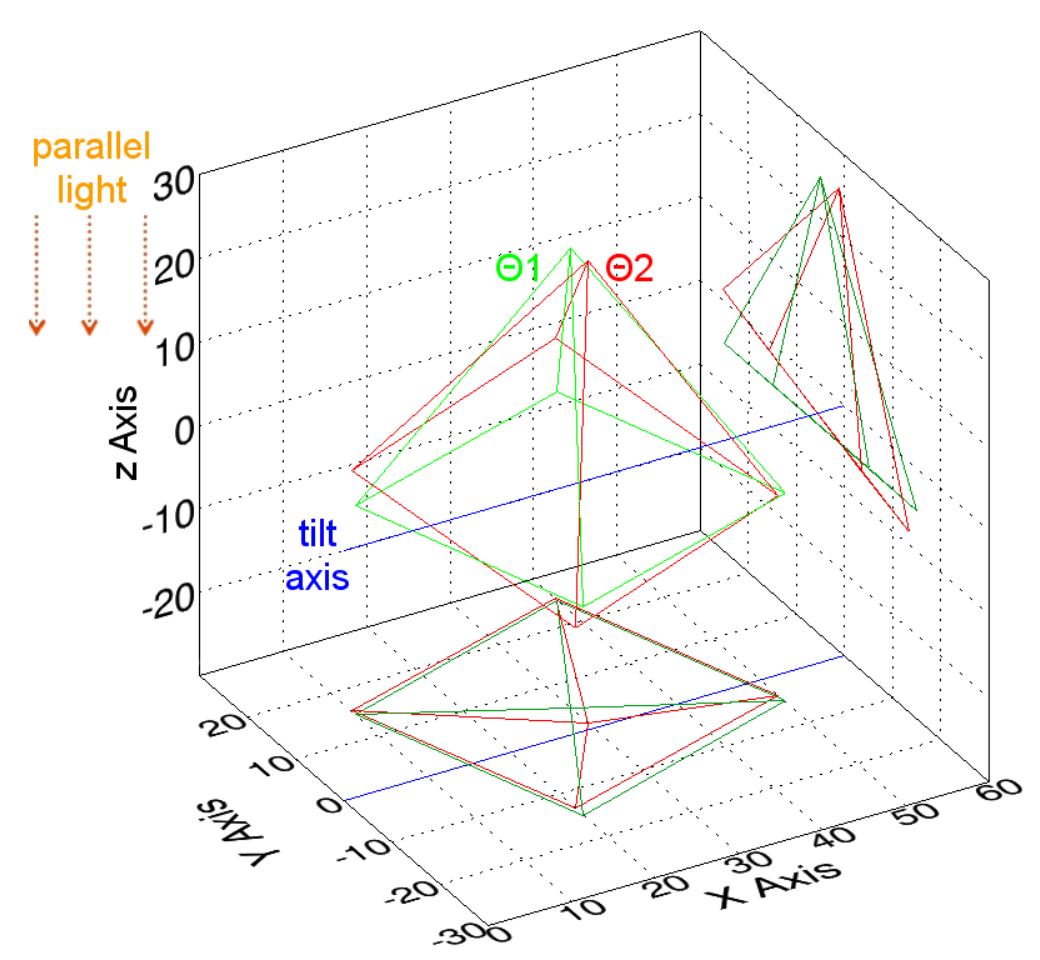

Fig. 3.6: Tilt by 14 degree of an object (pyramid) in three dimensions with the $x$-axis as rotational axis. The projection onto the $x-y$-plane demonstrates transmission images of the pyramid at both tilt angles with parallel illumination. Changes along the $y$-axis are revealed, while the $\mathrm{x}$-values are constant for corresponding object points. The projection onto the $y$-z-plane is shown to clarify the position of the pyramides and the tilt axis.

c are related as follows, where index 1 gives the coordinates of the projection of the counterclockwise tilted object, and index 2 of the clockwise tilted object with $\theta_{s}$ as tilting angle. The line with the length $s$ between a and $\mathrm{b}$ is parallel to the $\mathrm{x}$-axis, the line with the length $h$ between $\mathrm{b}$ and $\mathrm{c}$ is vertical to the $\mathrm{x}$-axis (the definition of the $\mathrm{x}$-axis as tilt axis is arbitrary).

$$
\begin{aligned}
\overline{a_{1} b_{1}} & =\overline{a_{2} b_{2}}=s \cos \theta_{\mathrm{s}} \text { and } \overline{c_{1} b_{1}}=\overline{b_{2} c_{2}}=h \sin \theta_{\mathrm{s}} \\
s_{1} & =\overline{a_{1} c_{1}}=s \cos \theta_{\mathrm{s}}-h \sin \theta_{\mathrm{s}} \quad \text { and } \quad s_{2}=\overline{a_{2} c_{2}}=s \cos \theta_{\mathrm{s}}+h \sin \theta_{\mathrm{s}} \\
\Rightarrow h & =\frac{s_{2}-s_{1}}{2 \sin \theta_{\mathrm{s}}}=\frac{\Delta Y}{2 \sin \theta_{\mathrm{s}}} \Leftrightarrow \quad \text { Parallax equation: } \Delta Y=2 h \sin \theta_{\mathrm{s}}
\end{aligned}
$$

More general and for a three dimensional object, the coordinates $(X, Y, Z)$ of one point are related as follows to the projection coordinates of that point in two images, $\left(x_{1}, y_{1}\right)$ and $\left(x_{2}, y_{2}\right)$, in which the object is projected onto the $\mathrm{x}$-y-plane and tilted by $\theta_{2}-\theta_{1}=\theta$ around a tilting axis parallel to the $\mathrm{x}$-axis (figure 3.6). The $\mathrm{z}$-coordinate of 
a structure can be calculated by the difference between the y-coordinates $\left(y_{1}\right.$ and $\left.y_{2}\right)$ in the respective images.

$$
\begin{aligned}
& {\left[\begin{array}{l}
x_{1} \\
y_{1}
\end{array}\right]=\left[\begin{array}{c}
X \\
Y \cos \theta_{1}+Z \sin \theta_{1}
\end{array}\right] \text { and }\left[\begin{array}{l}
x_{2} \\
y_{2}
\end{array}\right]=\left[\begin{array}{c}
X \\
Y \cos \theta_{2}+Z \sin \theta_{2}
\end{array}\right]} \\
& \theta=\theta_{2}-\theta_{1} \Leftrightarrow \theta_{1}=\theta / 2 \text { and } \theta_{2}=-\theta / 2 \\
& {\left[\begin{array}{l}
x_{1} \\
y_{1}
\end{array}\right]=\left[\begin{array}{c}
X \\
Y \cos (\theta / 2)+Z \sin (\theta / 2)
\end{array}\right] \text { and }\left[\begin{array}{c}
x_{2} \\
y_{2}
\end{array}\right]=\left[\begin{array}{c}
X \\
Y \cos (\theta / 2)-Z \sin (\theta / 2)
\end{array}\right]}
\end{aligned}
$$

The spatial coordinates become:

$$
X=x_{1}=x_{2} \quad Y=\frac{y_{1}+y_{2}}{2 \cos (\theta / 2)} \quad Z=\frac{y_{1}-y_{2}}{2 \sin (\theta / 2)}
$$

Thus, the reconstructed point is situated at the coordinates crossed on half way of its trajectory from its position when imaged first to its position after the tilt by $\theta$.

For human stereo vision, the parallax in a pair of images which fulfils the standard measures (DIN 4531 for stereoscopic images: image size of $54 \mathrm{~mm}$, distance between images of $8 \mathrm{~mm}$ and watching distance of $25 \mathrm{~mm}$ ) matches best when it is in the range of 3 to $5 \mathrm{~mm}$.

For micrographs, the magnification $M$ has to be taken into account to calculate the tilt angle for stereo vision from the parallax equation 3.1:

$$
\theta=2 \arcsin \left(\frac{\Delta Y}{2 M h}\right)
$$

However, human binocular vision is used to process images of objects opaque in visible light which hide everything behind them. Just the front of the object is visible, revealing also information about its surface. In transmission X-ray micrographs, all objects along the ray path are present as long as the absorption does not reach total absorption. This difference in the kind of information provided by the stereo images can lead to mistakes when looking at stereo X-ray micrographs or cause difficulties to achieve spatial perception.

Of course, the human image processor, the brain, is very complex and can be trained to handle transmission images easily. The impact of parallax is also strong enough to predominate over inconsistence with the spatial perception connected to other cognitive parameters like the eye accommodation, for example. Nevertheless, it is not a big effort to gain spatial information contained mathematically in a pair of transmission micrographs as shown in equation 3.2 via a computer programme (section 3.3 ). 


\subsubsection{Image alignment}

The positioning accuracy when tilting the objects is for most X-ray microscopes significantly worse than the resolution limit within the micrographs. When tilting, generally the object has to be focused again and often moved back to the centre of the image field. It is also possible that the tilt axis in the experimental setup is not perfectly parallel to the expected tilt axis, which is normally perpendicular to the illumination and to one of the image axis. Thus, relative movements of the projected structures within the set of only two micrographs occur, which have to be corrected by an image alignment.

For aligning the images of an X-ray tomographic data set, the orientation of the two-dimensional projection in the three-dimensional space is described by the alignment parameters. They take into account the tilt angle $\theta_{i}$ of the $i$ th projection perpendicular to the $\mathrm{x}$-axis, the in-plane translation $\mathbf{d}_{i}$ of the projection, the in-plane rotation angle $\alpha_{i}$, and the projection scale $s_{i}$. The projection $\mathcal{P}\left(\theta_{i}\right)$ of the object points $\mathbf{q}^{j}$ from three-dimensional space under the tilt angle $\theta_{i}$ is related to the respective positions $\mathbf{p}_{i}^{j}$ measured in the aligned two-dimensional projections as follows (Weiß, 2000 (94)):

$$
\mathcal{P}\left(\theta_{i}\right) \mathbf{q}^{j}=\mathcal{R}\left(\alpha_{i}\right) s_{i} \mathbf{p}_{i}^{j}+\mathbf{d}_{i}
$$

with the spatial coordinates of the $j$ th object point and its projected coordinates in the $i$ th projection

$$
\begin{aligned}
& \mathbf{x}^{j}=\left(\begin{array}{l}
x^{j} \\
y^{j} \\
z^{j}
\end{array}\right) \\
& \mathbf{p}_{i}^{j}=\left(\begin{array}{c}
p_{x_{i}}^{j} \\
p_{y_{i}}^{j}
\end{array}\right)
\end{aligned}
$$

and the respective transformations:

$$
\begin{aligned}
\mathcal{P}\left(\theta_{i}\right) & =\left(\begin{array}{ccc}
1 & 0 & 0 \\
0 & \cos \theta_{i} & -\sin \theta_{i}
\end{array}\right) \\
\mathcal{R}\left(\alpha_{i}\right) & =\left(\begin{array}{cc}
\cos \alpha_{i} & -\sin \alpha_{i} \\
\sin \alpha_{i} & \cos \alpha_{i}
\end{array}\right) \\
\mathbf{d}_{i} & =\left(\begin{array}{l}
d_{x_{i}} \\
d_{y_{i}}
\end{array}\right)
\end{aligned}
$$

The number of parameters is given by the alignment parameters $\theta_{i}, d_{x_{i}}, d_{y_{i}} \alpha_{i}, s_{i}$ times the number of projections $m$. As one of the tilt angles $\theta_{i}$ can be set to zero and one 
of the scale parameters $s_{i}$ to one, the number of parameters adds up to $5 m-2$. For a unique determination of all $5 m-2$ parameters, equation 3.4 has to be written as system of equations for at least three object points, for which the projection coordinates $\mathbf{p}_{i}^{j}$ can be measured in the micrographs. Therefore, fiducial markers within the sample environment are used formed by small spheres discernable in as much projection images as possible. Theoretically, at least three markers are necessary, but about ten should be available over the stack of tomographic projection images to obtain a good alignment result (Knöchel, 2005 (40)).

Details how a three-dimensional marker model is created by these alignment parameters and thereon an aligned tilt series can be found in Weiß, 2000 (94). There, the alignment is performed by the computer programme "XALIGN" developed in the Institute for X-ray physics, University Göttingen. The markers are set manually in each projection image.

A completely automated alignment of a tomographic set of X-ray micrographs is provided with the free software package "IMOD" (Mastronade, 1997 (49)). An application of "IMOD" in soft X-ray tomography is presented in (Parkinson et al., 2008 (60)).

Also X-ray stereo micrographs have to be aligned due to the reasons described above. But as a set of stereo images consists of only two images and the stereo reconstruction is treating distances, the required alignment is less complex.

A possible change in the projection scale $s_{i}$ can be neglected, as the object is focused for both micrographs and thus imaged with equal magnification.

Both in-plane translation $\mathbf{d}_{i}$ and in-plane rotation $\mathcal{R}\left(\alpha_{i}\right)$ are corrected as far as necessary by tilting and shifting the second stereo micrograph in such way that each object point has the same $\mathrm{x}$-value in both projection images. In xstereo this alignment is realised by alignment markers manually set in both micrographs (chapter 3.3). Therefore, the addition of fiducial markers to the sample is not needed.

A possibly remaining shift in y-direction has no influence on the stereo reconstruction. The distance calculations are invariant with regard to changes in the disparity of the stereo image pairs, i.e. a shift along the y-axis of one image relative to the other one. This can be clearly demonstrated for two object points $\mathrm{A}=\left(x^{a}, y^{a}, z^{a}\right)$ and $\mathrm{B}=\left(x^{b}, y^{b}, z^{b}\right)$ with the respective coordinates $\left(x_{1}^{a}, y_{1}^{a}\right),\left(x_{1}^{b}, y_{1}^{b}\right)$ in the stereo image 1 
taken before and $\left(x_{2}^{a}, y_{2}^{a}\right),\left(x_{2}^{b}, y_{2}^{b}\right)$ in the stereo image 2 taken after a tilt by the tilt angle $\theta$ around the $\mathrm{x}$-axis. The distance of these points is calculated by:

$$
\begin{aligned}
|\overline{a b}| & =\sqrt{\left(x^{a}-x^{b}\right)^{2}+\left(y^{a}-y^{b}\right)^{2}+\left(z^{a}-z^{b}\right)^{2}} \quad \text { |lequation 3.2 } \\
& =\sqrt{\left(x_{1}^{a}-x_{1}^{b}\right)^{2}+\left(\frac{y_{1}^{a}+y_{2}^{a}}{2 \cos (\theta / 2)}-\frac{y_{1}^{b}+y_{2}^{b}}{2 \cos (\theta / 2)}\right)^{2}+\left(\frac{y_{1}^{a}-y_{2}^{a}}{2 \sin (\theta / 2)}-\frac{y_{1}^{b}-y_{2}^{b}}{2 \sin (\theta / 2)}\right)^{2}} \\
& =\sqrt{\left(x_{1}^{a}-x_{1}^{b}\right)^{2}+\left(\frac{y_{1}^{a}+y_{2}^{a}-\left(y_{1}^{b}+y_{2}^{b}\right)}{2 \cos (\theta / 2)}\right)^{2}+\left(\frac{y_{1}^{a}-y_{2}^{a}-\left(y_{1}^{b}-y_{2}^{b}\right)}{2 \sin (\theta / 2)}\right)^{2}}
\end{aligned}
$$

For a shift of image 1 by a length $d$, the coordinates taken from image 1 become $\left(x_{1}^{a}, y_{1}^{a}+\right.$ $d),\left(x_{1}^{b}, y_{1}^{b}+d\right)$. Inserting the shifted coordinates to equation 3.10, the resulting distance does not change:

$$
\begin{aligned}
|\overline{A B}| & =\frac{\sqrt{\left(x_{1}^{a}-x_{1}^{b}\right)^{2}+\left(\frac{\left(y_{1}^{a}+d\right)+y_{2}^{a}-\left(\left(y_{1}^{b}+d\right)+y_{2}^{b}\right)}{2 \cos (\theta / 2)}\right)^{2}}}{} \\
& +\left(\frac{\left(y_{1}^{a}+d\right)-y_{2}^{a}-\left(\left(y_{1}^{b}+d\right)-y_{2}^{b}\right)}{2 \sin (\theta / 2)}\right)^{2} \\
= & \sqrt{\left(x_{1}^{a}-x_{1}^{b}\right)^{2}+\left(\frac{y_{1}^{a}+y_{2}^{a}-\left(y_{1}^{b}+y_{2}^{b}\right)}{2 \cos (\theta / 2)}\right)^{2}+\left(\frac{y_{1}^{a}-y_{2}^{a}-\left(y_{1}^{b}-y_{2}^{b}\right)}{2 \sin (\theta / 2)}\right)^{2}}
\end{aligned}
$$

Again, the images accord with the projections of the object onto the $\mathrm{x}-\mathrm{y}$-plane.

\subsubsection{Rotation axis}

For tomographic reconstruction, it is important to align all micrographs to one common tilt axis. However, for stereo reconstruction just the direction of the rotation axis in each image has to be known. This is verified as follows:

Two object points $\mathrm{A}$ and $\mathrm{B}$ with the respective coordinates $\left(x_{1}^{a}, y_{1}^{a}, z_{1}^{a}\right)$ and $\left(x_{1}^{b}, y_{1}^{b}, z_{1}^{b}\right)$ before and $\left(x_{2}^{a}, y_{2}^{a}, z_{2}^{a}\right)$ and $\left(x_{2}^{b}, y_{2}^{b}, z_{2}^{b}\right)$ after a tilt by the tilt angle $\theta$ around the $\mathrm{y}$-axis are given by:

$$
\begin{aligned}
& \left(\begin{array}{l}
x_{1}^{a} \\
y_{1}^{a} \\
z_{1}^{a}
\end{array}\right), \quad\left(\begin{array}{l}
x_{2}^{a} \\
y_{2}^{a} \\
z_{2}^{a}
\end{array}\right)=\left(\begin{array}{c}
x_{1}^{a} \\
y_{1}^{a} \cos \theta+z_{1}^{a} \sin \theta \\
-y_{1}^{a} \sin \theta+z_{1}^{a} \cos \theta
\end{array}\right) \\
& \left(\begin{array}{l}
x_{1}^{b} \\
y_{1}^{b} \\
z_{1}^{b}
\end{array}\right), \quad\left(\begin{array}{c}
x_{2}^{b} \\
y_{2}^{b} \\
z_{2}^{b}
\end{array}\right)=\left(\begin{array}{c}
x_{1}^{b} \\
y_{1}^{b} \cos \theta+z_{1}^{b} \sin \theta \\
-y_{1}^{b} \sin \theta+z_{1}^{b} \cos \theta
\end{array}\right)
\end{aligned}
$$


A tilt of an object by $\theta$ around a tilt axis parallel to the x-axis, but shifted by $d y$ in $\mathrm{y}$-direction and $d z$ in z-direction, is equal to a shift of the object to tilt by - $(0, d y, d z)$, then another tilt by $\theta$ around the x-axis and afterwards shift the tilted object back by $(0, d y, d z)$. For object points $\widetilde{\mathrm{A}}$ and $\widetilde{\mathrm{B}}$ with the same coordinates as $\mathrm{A}$ and $\mathrm{B}$ (equation 3.11) before the tilt, this yields:

$$
\begin{aligned}
& \left(\begin{array}{c}
x_{1}^{a} \\
y_{1}^{a} \\
z_{1}^{a}
\end{array}\right) \stackrel{\text { shift }}{\longrightarrow}\left(\begin{array}{c}
x_{1}^{a} \\
y_{1}^{a}-d y \\
z_{1}^{a}-d z
\end{array}\right) \stackrel{\text { rotation }}{\longrightarrow}\left(\begin{array}{c}
x_{1}^{a} \\
\left(y_{1}^{a}-d y\right) \cos \theta+\left(z_{1}^{a}-d z\right) \sin \theta \\
-\left(y_{1}^{a}-d y\right) \sin \theta+\left(z_{1}^{a}-d z\right) \cos \theta
\end{array}\right) \text { shiftback } \\
& \left(\begin{array}{c}
x_{1}^{a} \\
\left(y_{1}^{a}-d y\right) \cos \theta+\left(z_{1}^{a}-d z\right) \sin \theta+d y \\
-\left(y_{1}^{a}-d y\right) \sin \theta+\left(z_{1}^{a}-d z\right) \cos \theta+d z
\end{array}\right)=\left(\begin{array}{c}
\widetilde{x}_{2}^{a} \\
\widetilde{y}_{2}^{a} \\
\widetilde{z}_{2}^{a}
\end{array}\right) \\
& \left(\begin{array}{c}
x_{1}^{b} \\
y_{1}^{b} \\
z_{1}^{b}
\end{array}\right) \stackrel{\operatorname{shift}}{\longrightarrow}\left(\begin{array}{c}
x_{1}^{b} \\
y_{1}^{b}-d y \\
z_{1}^{b}-d z
\end{array}\right) \stackrel{\text { rotation }}{\longrightarrow}\left(\begin{array}{c}
\left(y_{1}^{b}-d y\right) \cos \theta+\left(z_{1}^{b}-d z\right) \sin \theta \\
-\left(y_{1}^{b}-d y\right) \sin \theta+\left(z_{1}^{b}-d z\right) \cos \theta
\end{array}\right) \text { shiftback } \\
& \left(\begin{array}{c}
x_{1}^{b} \\
\left(y_{1}^{b}-d y\right) \cos \theta+\left(z_{1}^{b}-d z\right) \sin \theta+d y \\
-\left(y_{1}^{b}-d y\right) \sin \theta+\left(z_{1}^{b}-d z\right) \cos \theta+d z
\end{array}\right)=\left(\begin{array}{c}
\widetilde{x}_{2}^{b} \\
\widetilde{y}_{2}^{b} \\
\widetilde{z}_{2}^{b}
\end{array}\right)
\end{aligned}
$$

The distance between $\widetilde{\mathrm{A}}$ and $\widetilde{\mathrm{B}}$ calculated by following equation 3.10 with $\widetilde{y}_{2}^{a}$ instead of $y_{2}^{a}$, and $\widetilde{y}_{2}^{b}$ instead of $y_{2}^{b}$ becomes:

$$
|\overline{\widetilde{A} \widetilde{B}}|=\sqrt{\left(x_{1}^{a}-x_{1}^{b}\right)^{2}+\left(\frac{y_{1}^{a}+\widetilde{y}_{2}^{a}-\left(y_{1}^{b}+\widetilde{y}_{2}^{b}\right)}{2 \cos (\theta / 2)}\right)^{2}+\left(\frac{y_{1}^{a}-\widetilde{y}_{2}^{a}-\left(y_{1}^{b}-\widetilde{y}_{2}^{b}\right)}{2 \sin (\theta / 2)}\right)^{2}}
$$




$$
\begin{aligned}
& \text { with } \quad y_{1}^{a}+\widetilde{y}_{2}^{a}-\left(y_{1}^{b}+\widetilde{y}_{2}^{b}\right) \\
& =y_{1}^{a}+\left(y_{1}^{a}-d y\right) \cos \theta+\left(z_{1}^{a}-d z\right) \sin \theta+d y \\
& -\left(y_{1}^{b}-\left(y_{1}^{b}-d y\right) \cos \theta+\left(z_{1}^{b}-d z\right) \sin \theta+d y\right) \\
& =y_{1}^{a}+y_{1}^{a} \cos \theta-d y \cos \theta+z_{1}^{a} \sin \theta-d z \sin \theta+d y \\
& -\left(y_{1}^{b}-y_{1}^{b} \cos \theta-d y \cos \theta+z_{1}^{b} \sin \theta-d z \sin \theta+d y\right) \\
& =y_{1}^{a}+y_{1}^{a} \cos \theta+z_{1}^{a} \sin \theta-\left(y_{1}^{b}-y_{1}^{b} \cos \theta+z_{1}^{b} \sin \theta\right) \\
& =y_{1}^{a}+y_{2}^{a}-\left(y_{1}^{b}-y_{2}^{b}\right) \\
& \text { and } \quad y_{1}^{a}-\widetilde{y}_{2}^{a}-\left(y_{1}^{b}-\widetilde{y}_{2}^{b}\right) \\
& =y_{1}^{a}-\left(\left(y_{1}^{a}-d y\right) \cos \theta+\left(z_{1}^{a}-d z\right) \sin \theta+d y\right) \\
& -\left(y_{1}^{b}-\left(\left(y_{1}^{b}-d y\right) \cos \theta+\left(z_{1}^{b}-d z\right) \sin \theta+d y\right)\right) \\
& =y_{1}^{a}+y_{1}^{a} \cos \theta+d y \cos \theta-z_{1}^{a} \sin \theta+d z \sin \theta-d y \\
& -y_{1}^{b}+y_{1}^{b} \cos \theta-d y \cos \theta+z_{1}^{b} \sin \theta-d z \sin \theta+d y \\
& =y_{1}^{a}-y_{1}^{a} \cos \theta-z_{1}^{a} \sin \theta-y_{1}^{b}+y_{1}^{b} \cos \theta+z_{1}^{b} \sin \theta \\
& =y_{1}^{a}-\left(y_{1}^{a} \cos \theta+z_{1}^{a} \sin \theta\right)-\left(y_{1}^{b}-\left(y_{1}^{b} \cos \theta+z_{1}^{b} \sin \theta\right)\right) \\
& =y_{1}^{a}-y_{2}^{a}-\left(y_{1}^{b}-y_{2}^{b}\right) \\
& \begin{aligned}
\Rightarrow \quad \overline{\widetilde{A} \widetilde{B}} \mid & =\sqrt{\left(x_{1}^{a}-x_{1}^{b}\right)^{2}+\left(\frac{y_{1}^{a}+y_{2}^{a}-\left(y_{1}^{b}+y_{2}^{b}\right)}{2 \cos (\theta / 2)}\right)^{2}+\left(\frac{y_{1}^{a}-y_{2}^{a}-\left(y_{1}^{b}-y_{2}^{b}\right)}{2 \sin (\theta / 2)}\right)^{2}} \\
& =|\overline{A B}|
\end{aligned}
\end{aligned}
$$

Thus, the distance between $\mathrm{A}$ and $\mathrm{B}$ is the same as between $\widetilde{\mathrm{A}}$ and $\widetilde{\mathrm{B}}$ when calculated using both times equation 3.10. This shows that the distance between two object points can be calculated from a pair of stereo images aligned with regard to the x-axis, as if they would have been tilted around the $\mathrm{x}$-axis while the tilt axis in the experiment just has to be parallel to the $\mathrm{x}$-axis.

\subsubsection{Resolution limit}

The theoretical resolution limit for measuring a distance in z-direction of two points from stereo images is described by the achievable depth resolution (see section 2.2.1) in a pair of stereo micrographs. Let us assume that the tilt angle and the magnification 
$M$ of the microscopic experiment are sufficiently well known. Then the Gaussian error propagation yields to an accuracy of measuring the parallax, which means the position of two points per stereo image, of:

$$
\sigma(\Delta Y)=2 M \delta_{l}
$$

where $\delta_{l}$ is the lateral resolution (section 2.2.1). Inserting this into equation 3.1, the accuracy of measuring $h$ from stereo images becomes

$$
\sigma(h)=\frac{2 \delta_{l}}{2 \sin (\theta / 2)} \approx \frac{2 \delta_{l}}{|\theta|}
$$

The results from xstereo depend on the accuracy when marking the structures, but can be assumed to be within a range of two to three pixel for images with sufficiently good contrast.

\subsection{Programme xstereo}

The programme xstereo has been written in IDL to analyse pairs of stereo X-ray micrographs based on the stereo reconstruction described in chapter 3.2. The xstereo user interface is shown in figure 3.7. The work steps are called in particular via the buttons on the left side. The interaction with the programme is given via four text fields below the buttons. Three image frames allow for clear image editing as both micrographs of the stereo pair are permanently shown in their actual processing status in two smaller frames below while the processing takes place in the main image frame. To enable exact positioning of markers, the micrograph in the main image can be zoomed for processing, and the actual cursor position in the image is displayed. The "Zoom" button allows also for zooming the overview images shown in the smaller frames, which are by default scaled to the frame size. As additional feature for setting the respective markers with high accuracy, the reference stereo image can be opened in a separate window with zoom and cursor position detection via the button "Open reference image frame".

The work steps provided by the buttons on the left side of the interface offer to load stereo images, align images, set stereo markers, load a stereo file, make a threedimensional plot of the stereo markers, and calculate distances. The buttons are arranged in logical order, but can be used as requested.

To start from the beginning, it is necessary to load a pair of stereo micrographs from two separate files in tiff format and enter the total tilt angle in degree. The tilt axis has 


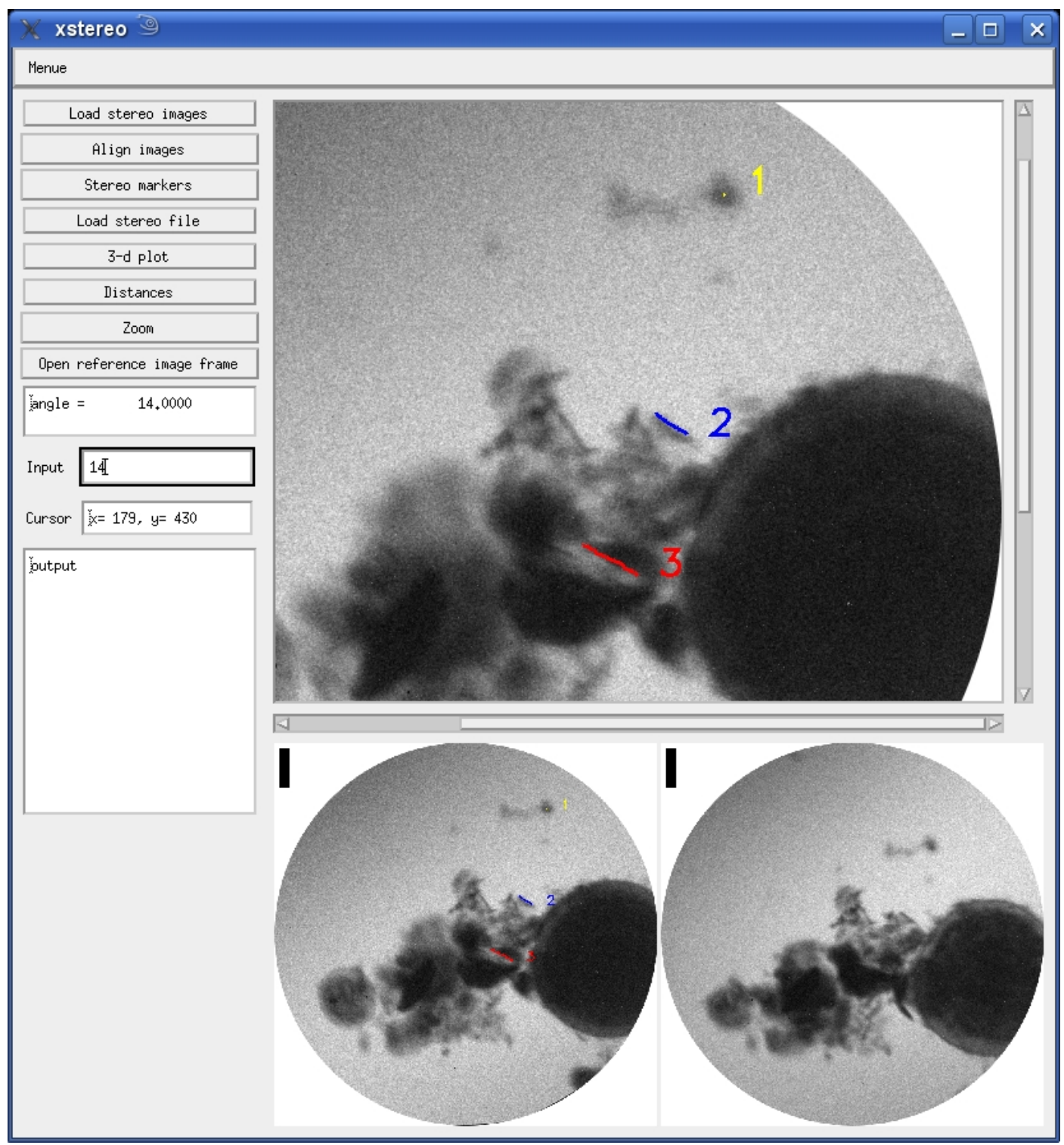

Fig. 3.7: Graphical user interface of the xstereo programme. The buttons on the left side provide several steps of stereo image processing. Four text boxes are situated below for short current informations like input requests, input by the user, current cursor position, and a large field for the output of calculated distances. On the right side below, the pair of stereo micrographs is shown in its most actual processing status in two overview frames, while the manual processing is done in the big image frame on the top right. The stereo images are called successively to the work frame with the option to zoom for precise marker positioning. The three-dimensional plot of the marked structures is shown in a separate window (figure 3.8). 
to be parallel to the horizontal axis, in the following called $\mathrm{x}$-axis which is defined as to be centered. Both images appear in the frames below. The next step is optionally the alignment of the images if necessary. To do so, the "Align images" button opens eight sub-buttons to choose between setting, adding, or deleting alignment markers in either the left or the right image, execute the alignment on the markers set previously, and save the aligned images. The image chosen to set marker to appears in the main image frame where it can be zoomed. The markers are set with pressing the left mouse button and appear as red dots with the identification number right besides. Finally, each marker has to be set in both images, each time with the same identification number. The alignment is executed by tilting the right side stereo micrograph so that each alignment marker is positioned on the same $\mathrm{x}$-value as in the left stereo image, corresponding to the argumentation given in chapter 3.2.2.

With a pair of aligned micrographs, the procedure for setting stereo markers is to be chosen via the button "Stereo markers". They are set in a similar way as the alignment markers, but with the additional possibility to draw lines as well by keeping the right mouse button pressed. Again, the identification number of the stereo structures has to be congruent in both micrographs, which is of significance for the correlation of the markers in the stereo reconstruction. A point structure is set automatically to the $\mathrm{x}$ value of the marker set in the left micrograph, as long as the difference is not exceeding 10 pixels, which would disqualify the two points from being matched. For drawn structures, the reconstruction of the spatial coordinates works also point-wise and is done per pair of points with equal x-values in both stereo images. If the structure crosses one $\mathrm{x}$ value several times, that marker point from the right image is associated which has the smallest distance to the previously correlated point of the same structure. This method proved to be successful for structures still foremost extended in the x-direction. For a vertical structure the reconstruction achieves better results when it is marked with two single points at its ends and connected to a line afterwards. Yet this is only allowable if the single points mark the same spot of the structure, i.e. a corner recognisable in both micrographs or a distinctive spot of an edge.

The set of stereo markers can be saved along with the (aligned) initial images, the tilt angle and optionally a comment. The data is saved by a filename query in the IDL specific "sav" format. Such file is required by the "Load stereo file" button, so further processing of stored data is possible. Next, the three-dimensional plot can be selected, by what the stereo markers are collated and the spatial coordinates of each pair of markers are calculated relative to a centered tilt axis coinciding with the $\mathrm{x}$-axis. The 


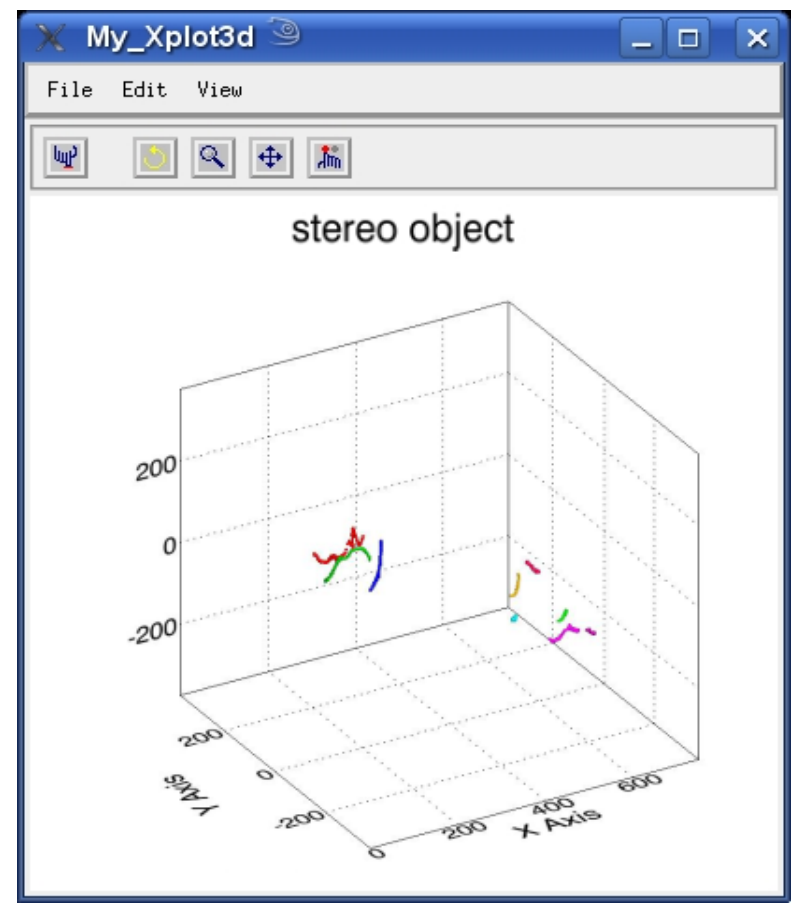

Fig. 3.8: The plot tool "My_Xplot3d" implemented in xstereo is used to visualise the spatial arrangement of the features marked previously in the stereo images. It is based on the IDL tool Xplot3d, but extended to the "Make movie" function, which enables to make a movie of the plot with adjustable number of frames, tilts and zoom.

spatial arrangement of these structures is depicted in a three-dimensional plot which opens in a window apart. The custom-designed plot tool "My_Xplot3d" implemented in xstereo is based on the IDL tool "Xplot3d", but extended to the "make movie" function, which enables to make a movie of the plot with adjustable number of frames, tilts and zoom. This extension was realised in-house by Gudrun Gleber. The window of "My_Xplot3d" is shown in figure 3.8 presenting a view of the three-dimensional plot as a result of xstereo processing. Both movies and single images of views of the plot adjustable at will in zoom, tilt and shift can be saved. The format for the movies is ".gif" and for the images selectively ".gif", ".tif" or ".jpg".

As last step of the stereo processing provided by xstereo distances are calculated. When selected, the dedicated subbutton starts twice a query in the upper text box of an identification number of a structure and thereon displays the minimum distance between the selected structures in the large text box. The default unit is pixel, but is converted automatically if the pixel size is given as optional entry via a subbutton within the "Distances" and the "3-d plot" procedure. Several distances can be calculated 
consecutively. A compilation of these results can be saved together with images of both micrographs including the stereo markers set on it as images in the ".tif" format. 


\section{Chapter 4}

\section{Soft X-ray microscopes}

Four soft X-ray microscopy setups were used for the experiments within the scope of this work, the XM-1 at the ALS, the CSXM at the KTH, the STXM at BESSY II, and the TwinMic at ELETTRA. The combination of stereo microscopy with both elemental mapping and sample manipulation is performable at two of the four instruments, the XM-1 and the STXM. With the XM-1 and the TwinMic, elemental mapping of iron is possible. The STXM can be used for elemental mapping and spectroscopy at the carbon K-absorption edge. The CSXM is not utilising synchrotron radiation, but Xradiation provided by a compact laser plasma source. As line radiation with a very narrow bandwidth is used as a light source, elemental mapping or spectroscopy are not possible, however, the approach of a table-top light source and microscope is extremely promising for X-ray imaging experiments in one's own laboratory.

\subsection{Full-field soft X-ray microscopes}

A full-field transmission soft X-microscope consists usually of a condenser zone plate and a high-resolution micro zone plate (chapter 2.2). The main advantage of full-field microscopes compared with scanning microscopes is the relatively short exposure time, at synchrotron-based microscopes in the range of milliseconds or seconds, for imaging a large sample region of several micrometres.

\subsubsection{XM-1 at ALS}

The high resolution full field soft X-ray transmission microscope of the Center for XRay Optics located at beamline 6.1.2 of the Advanced Light Source, Lawrence Berkeley Laboratory, in Berkeley, USA, has been used (Meyer-Ilse et al., 2000 (50); Fischer et 


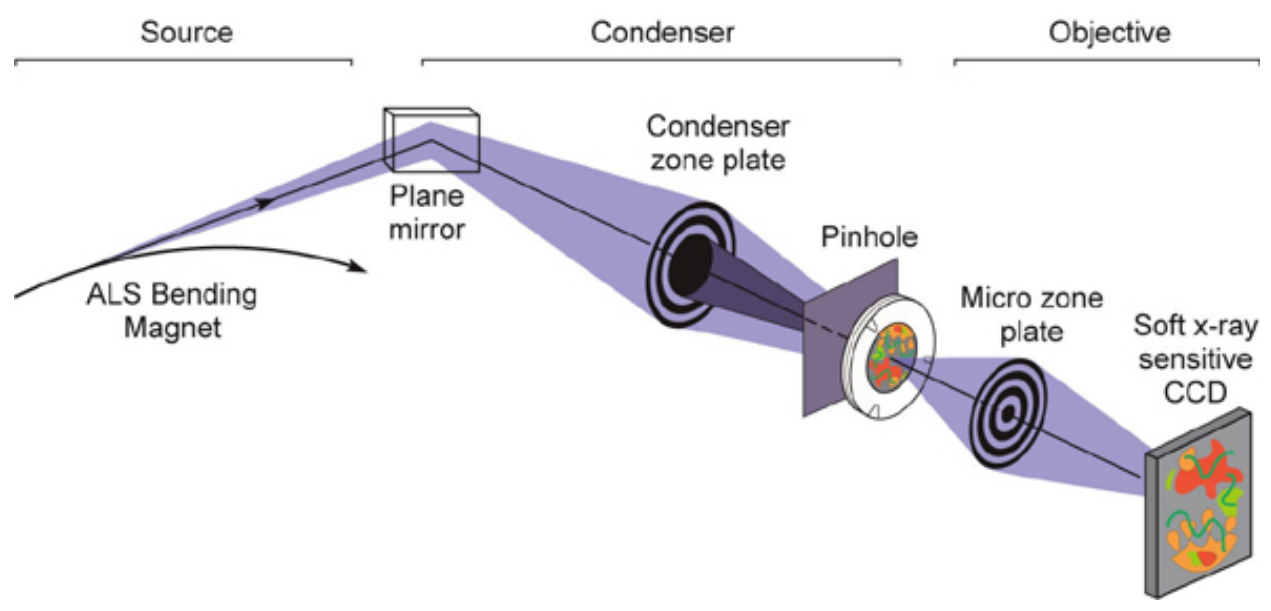

Fig. 4.1: Scheme of the X-ray microscope XM-1 beamline at the ALS. [From Attwood, 1999 (5)]

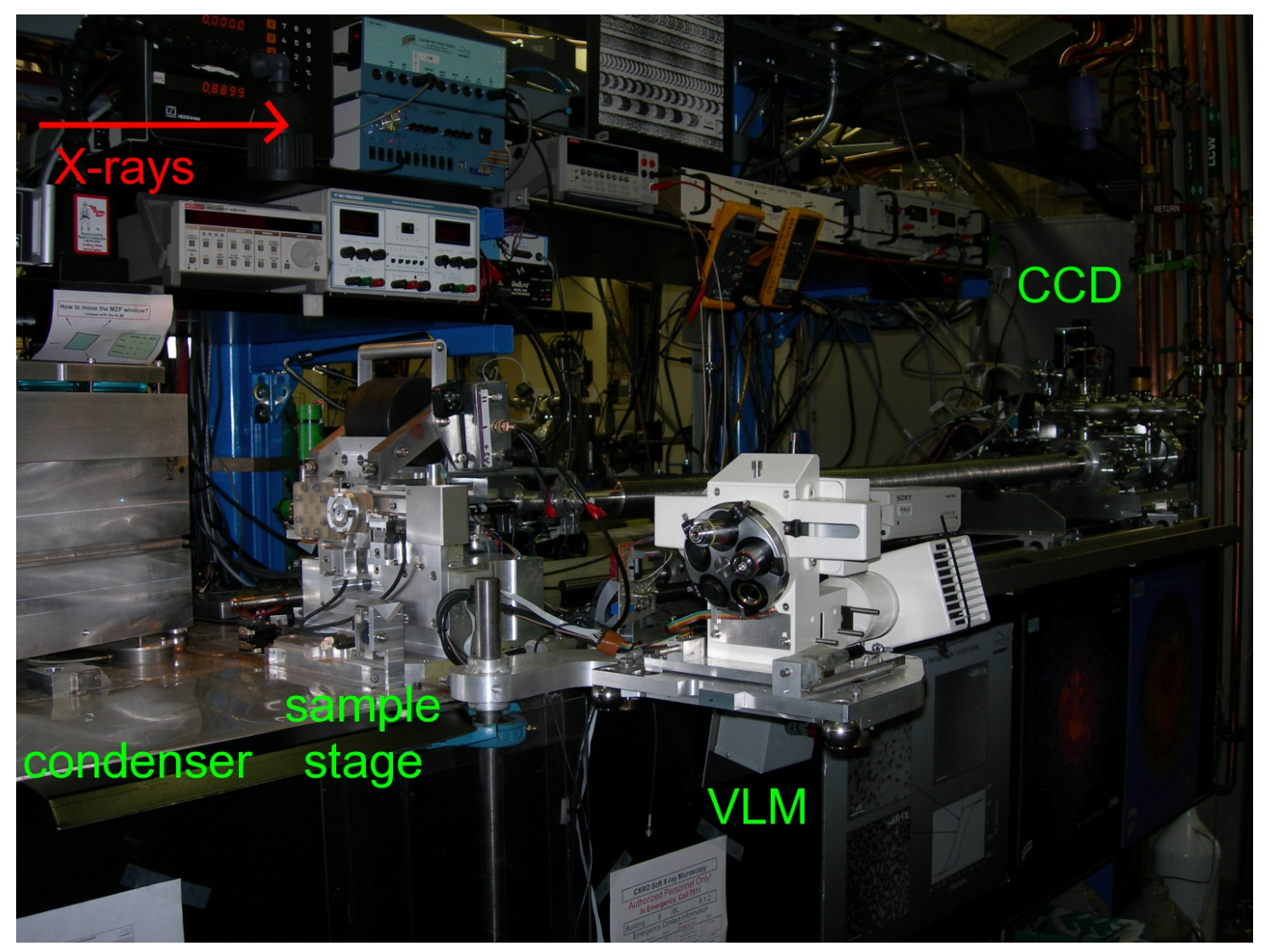

Fig. 4.2: Photograph of the $X$-ray microscope XM-1 at the ALS. The vacuum vessel with the condenser zone plate is removed giving access to the sample stage. The visible light microscope can be moved to the sample stage. 
al., 2006 (18); Thieme et al., 2003 (88)). In figure 4.1, the X-ray optical set-up of this transmission X-ray microscope is shown, consisting of Fresnel zone plates for both the condenser optics and the micro optics. The condenser zone plate collects the synchrotron radiation emitted from a bending magnet and focuses it onto the object. In addition, in combination with the pinhole in front of the object it works as a linear monochromator (Niemann et al., 1974 (56)). It reduces the bandwidth of the radiation illuminating the object to about $\mathrm{E} / \Delta \mathrm{E} \approx 500$. A micro zone plate downstream the object acts as high resolution X-ray objective and forms an enlarged image of the object in the image plane. The image is recorded with a backside illuminated slow-scan CCD-camera, where electron-hole pairs are liberated by the descending photons. These electrons produce a signal in counts according to a camera specific transfer rate, proportional to the incident photon energy. Exposure times are typically in the range of several seconds. For the experiments, a micro zone plate with an outermost zone width of $d r_{n}=25 \mathrm{~nm}$ was used to take high resolution images around the Fe-L edge at approximately $\mathrm{E}=707 \mathrm{eV}$ or $\lambda=1.75 \mathrm{~nm}$. According to equation 2.15, the lateral resolution is $\delta_{l}=1.22 d r_{n} \approx 30 \mathrm{~nm}$. Following equation 2.20, the depth of focus in the experiments has been $\delta \approx 1.43 \mu \mathrm{m}$ using the first diffraction order $(\mathrm{m}=1)$ (Attwood, $1999(5))$, but not considering the depth of focus elongation due to a narrow-bandwidth illumination. The magnification was about 2800. The CCD detector (Princeton Instruments) has been used either with 2048 x $2048 \mathrm{pxl}^{2}$ or in a $1024 \times 1024 \mathrm{pxl}^{2}$ binned mode, resulting in a pixel length of either $4.5 \mathrm{~nm}$ or $9 \mathrm{~nm}$ in the object plane. Thus, the images have been sufficiently oversampled with regard to the optical resolution.

Due to the high absorption in air of X-rays with such a low energy with a $1 / e$ absorption length of about $1.2 \mathrm{~mm}$, the optical path has to be in vacuum. To allow for samples in aqueous media, the sample stage is therefore located in a small air gap between the vacuum chamber containing the condenser and the vacuum chamber holding the micro zone plate and the CCD camera (figure 4.2). To optimise between X-ray transmission and sample handling, the gap is typically about $200 \mu \mathrm{m}$ wide. Both vacuum chambers are sealed with $\mathrm{Si}_{3} \mathrm{~N}_{4}$ windows of typically $100 \mathrm{~nm}$ thickness. To access the sample stage, the condenser vacuum chamber is removed. By switching in a visible light microscope (VLM), the sample can be prealigned and a region of interest can be selected for X-ray imaging.

For the performance of stereo experiments, the XM-1 object stage was slightly adapted, so the detachable tilt stage could be implemented (chapter 3.1, figure 3.1). For these first experiments, tilting was performed manually. Each tilt position was 


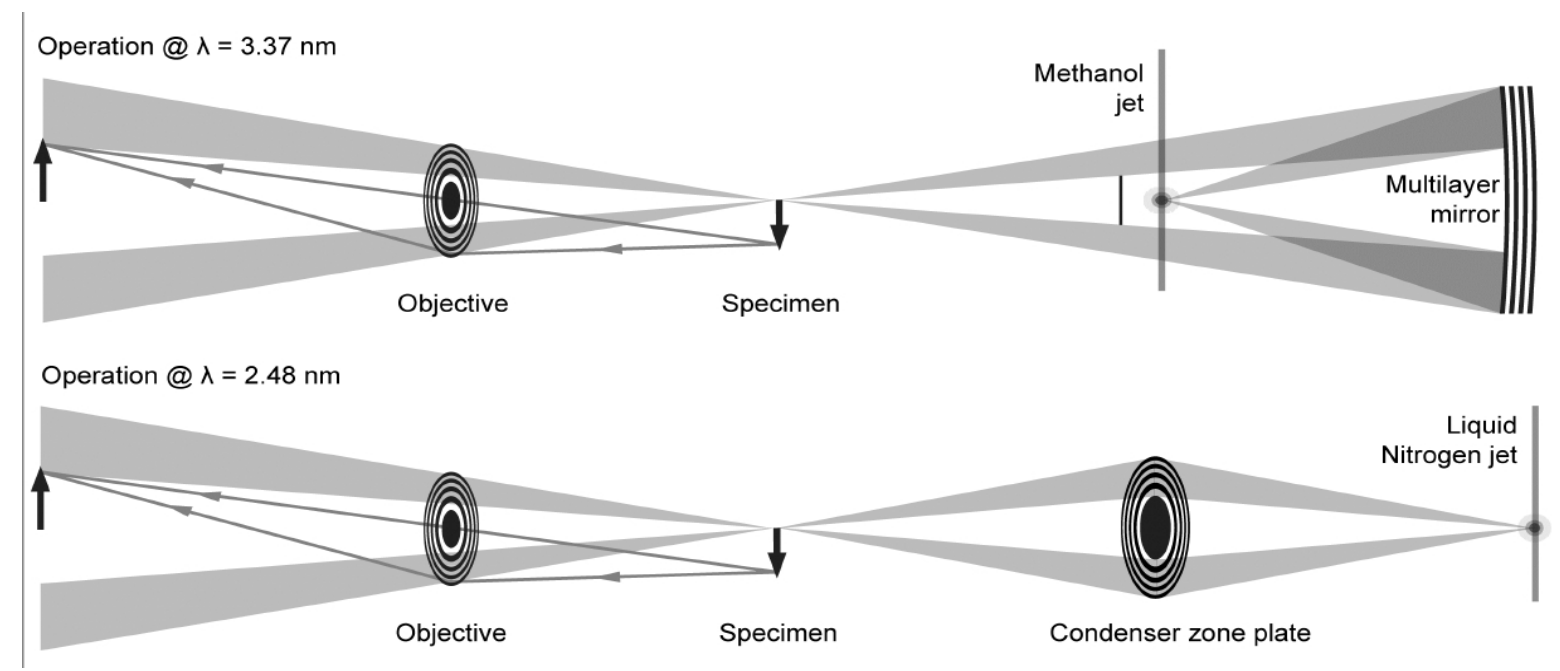

Fig. 4.3: Scheme of the compact soft X-ray microscope at the KTH, Stockholm. Above: Setup for the operation at $\lambda=3.37 \mathrm{~nm}$. Below: Setup for the operation at $\lambda=2.24 \mathrm{~nm}$. [From Bertilson et al., 2009 (8)]

recorded with a digital foto camera imaging the position of the cog wheel, and the tilt angle was determined by measuring the difference between the respective cog wheel positions in the images. The estimated accuracy for the resulting tilt angle is $0.5^{\circ}$.

\subsubsection{Compact soft X-ray microscope at KTH}

In order to provide soft X-ray microscopy as an off synchrotron laboratory method, Hertz and his group at the Royal Institute of Technology (KTH, Kungliga Tekniska Högskolan), Stockholm, Sweden, developed the first full-field soft X-ray microscope based on a compact liquid-jet laser-plasma source (Hertz et al., 2000 (28) and Berglund et al., 2000 (7)). Brightness of compact X-ray sources and efficiency of soft X-ray optics have increased enough over the last years that compact X-ray microscopes improved their imaging properties tremendously.

The light source of the compact soft X-ray microscope (CSXM) at the KTH is an $\mathrm{X}$-ray emitting plasma. It is generated by a pulsed laser of approximately $3 \mathrm{~ns}$ puls length, $100 \mathrm{~Hz}$ frequency and $100 \mathrm{~mJ}$ energy, which is focused onto a liquid jet target. Depending on the target, the microscope can be operated at different energies within the range of the water-window, thus very well suitable for aqueous samples. A methanol target emits X-rays of $\lambda=3.37 \mathrm{~nm}$ wavelength (Berglund et al., 2000 (7)), and a liquid nitrogen target emits at $\lambda=2.48 \mathrm{~nm}$ wavelength (Jansson et al., 2005 (34), Takman et al., $2007(82)$ ). The achievable photon flux is $10^{11}$ to $10^{12}$ photons/(pulse $\cdot \mathrm{sr} \cdot$ line) and 


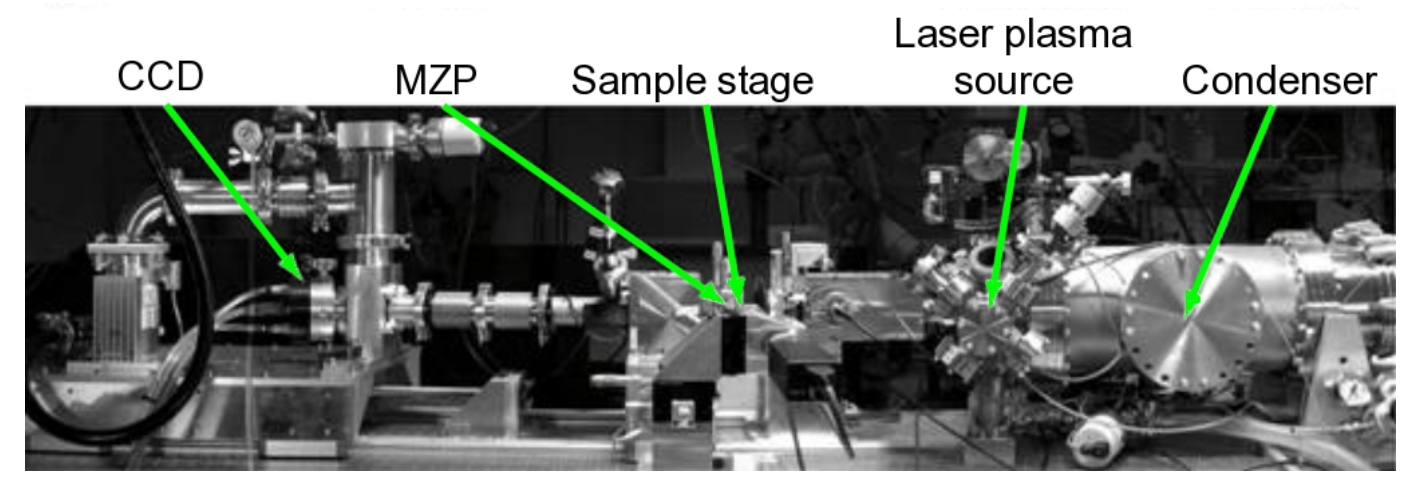

Fig. 4.4: Photograph of the Compact soft X-ray microscope (CSXM) at the KTH. [Adapted from www.biox.kth.se/research/xraymicroscopy/xrmproject.htm]

the line monochromaticity $\lambda / \Delta \lambda>500$ (Bertilson et al., 2009 (8)). When operating with a methanol jet, the light of $2.48 \mathrm{~nm}$ wavelength is focused onto the sample by a chromium/scandium multilayer mirror condenser with $58 \mathrm{~mm}$ diameter and $3.12 \%$ average reflectibility. The $3.37 \mathrm{~nm}$ illumination from the operation with a liquid nitrogen jet is focused with a condenser zone plate of $4.53 \mathrm{~mm}$ diameter and $49 \mathrm{~nm}$ outermost zone width directly onto the sample. The sample stage is positioned in a helium atmosphere to provide an improved transmissibility compared to air, but maintaining room pressure conditions.

With in-house fabricated nickel zone plates of $25 \mathrm{~nm}$ outermost zone width as objectives, the compact X-ray microscope provides sub-30 nm resolution (Takman et al., 2007 (82)). The image is detected by a $2048 \times 2048 \mathrm{pxl}^{2}$ CCD detector (Princeton Instruments, USA) with a pixel size of $13 \mu \mathrm{m}$.

The presented experiments were performed using a $30 \mathrm{~nm}$ zone plate and $3.37 \mathrm{~nm}$ wavelength $(368 \mathrm{eV})$, resulting in a numerical aperture of about 0.056 . The depth of focus after equation 2.19 is about $0.65 \mu \mathrm{m}$, considering monochromatic illumination. The measured resolution in the presented micrographs is $50 \mathrm{~nm}$, where the exposure times are in the range of $4 \mathrm{~min}$ to $10 \mathrm{~min}$. Due to the relatively long exposure time, possible sample motion has to be considered. For this purpose, images are taken subsequently as image series, with single images of $60 \mathrm{sec}$ exposure time each.

The stereo mount provides a tilt of $+/-7^{\circ}$ relative to the image plane perpendicular to the X-rays and thus parallel to the projection plane. By this, flat structures parallel to the $\mathrm{Si}_{3} \mathrm{~N}_{4}$ membrane do not show any parallax in the stereo image pair. As the projection images are transmission images, flat structures look the same in both images, 
unless the focus setting is similar and the structures are not as much extended as they exceed the depth of focus when tilted by $7^{\circ}$.

The first tomographic data set taken at a compact X-ray microscope is presented in (Bertilson et al., 2009 (8)). Towards biological applications of this technique, the implementation of a cryo stage is planned (Hertz et al., 2009 (29)).

The first investigations with soil samples were performed by Thieme et al., 2009 (89), and in the scope of this work (Gleber et al., 2009 (21)).

\subsection{Scanning soft X-ray microscopes}

Scanning transmission X-ray microscopes are capable of taking X-ray absorption spectra with both high spectral and high spatial resolution (chapter 2.4). The condenser optic focuses highly monochromatic X-radiation onto the sample, where the spot size determines the spatial resolution. After transmission of the sample, the X-radiation is recorded directly by a CCD camera. Due to the lack of a micro zone plate, generally with an efficiency of less than $10 \%$, the dosage applied to the sample is about one order of magnitude lower than for full-field microscopes. To record an image, the sample has to be scanned. The scanning process is perfomed either by moving the sample, or the condenser zone plate. This process is more time consuming than imaging in full-field.

\subsubsection{STXM at BESSY}

The STXM at the electron storage ring BESSY II, in Berlin, Germany, is located at the undulator beamline U 41-XM, which serves as a coherent light source (Wiesemann, 2003 (96) and Wiesemann et al., 2003 (97)). Since 2007, the STXM is operated with a new BESSY monochromator, a focusing spherical grating monochromator (FSGM), providing a monochromaticity of up to 10000, resulting in a spectral resolution of the STXM of about 3000 (Guttmann et al., 2008 (25)). First images taken with this new setup were performed in the scope of this work (chapter 6). The FSGM can be operated in a wide energy range from $161 \mathrm{eV}$ to $1850 \mathrm{eV}$. However, the undulator U 41 does not exceed $600 \mathrm{eV}$ with the first harmonic. For achieving higher energies, higher harmonics have to be used, which means a loss in photon rate.

In the STXM, a Fresnel zone plate is employed as high resolution optical element to create a diffraction-limited focus at the sample plane. The zone plate is mounted on a piezo-electric scanner, permitting its fine movement in $\mathrm{x}$-y-direction perpendicular to the X-ray beam direction. Zone plate and piezo scanner are in a high vacuum of 


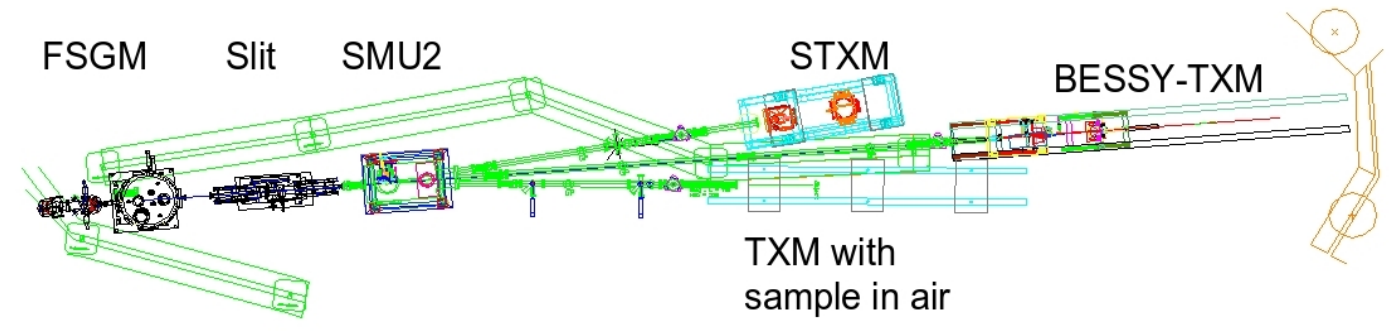

Fig. 4.5: Scheme of the U41 beamline at BESSY with the new focusing spherical grating monochromator and the three end-stations STXM, new TXM, and TXM with the sample in air. [From Guttmann et al., 2008 (25)]

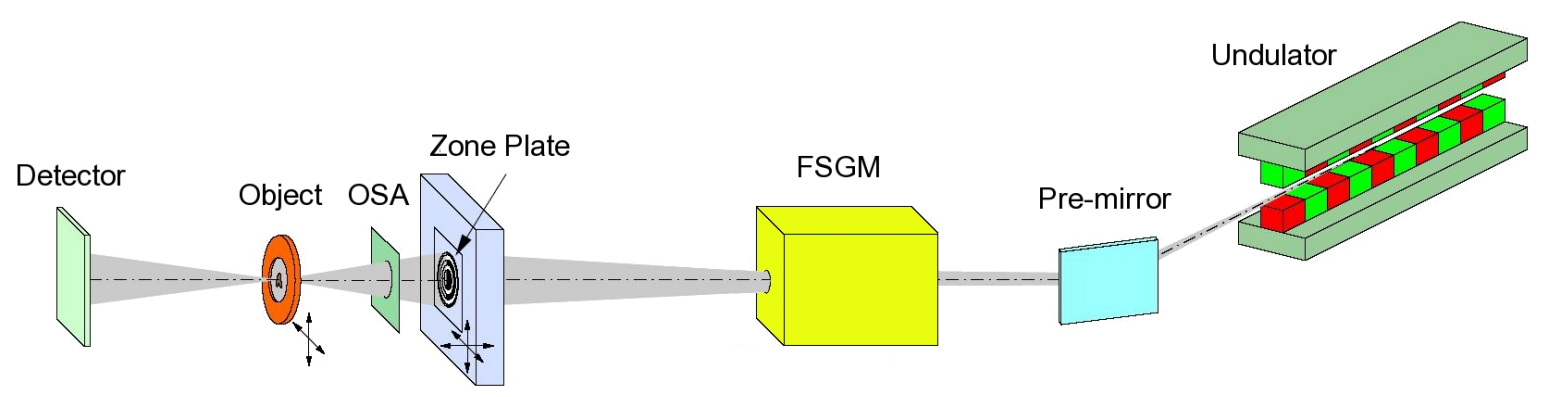

Fig. 4.6: Schematic of the Scanning Transmission X-ray microscope at BESSY with an undulator source, a pre-mirror, a focusing spherical grating monochromator (FSGM), a high-resolution zone plate, an order-sorting aperture (OSA), an object stage, and a CCD detector. The zone plate is movable in three directions, the object stage is movable in two directions perpendicular to the optical axis (dash-dotted line). [Adapted from Wiesemann, $2003(96)]$ 
$10^{-8}$ mbar. An order-sorting aperture (OSA) of a $40 \mu \mathrm{m}$ radius is placed in between the sample stage and zone plate stage. It is adapted to let pass only the light cone of the first diffraction order for illumination of the sample, while it blocks the light from zeroth and higher diffraction orders. In addition, the OSA acts as a vacuum window. A manual adjustment of the OSA in $\mathrm{x}$ - and $\mathrm{y}$ - direction is possible to match it with the numerical aperture of the zone plate. With stepper motors the sample can be coarsely moved in $\mathrm{x}$ - and $\mathrm{y}$ - directions to reach a pre-alignment for a region of interest. This is done under observation with a visible light microscope (VLM) which is moved in. By focusing with the VLM, the distance between the OSA and the sample is gained. As the OSA is fixed and its focus is known after imaging it with the STXM, an estimation of the STXM focus for the sample is made, which has an accuracy in the range of a few micrometres. The intensity transmitted through the sample is recorded by a pn-CCD detector (Ketek GmbH, München, Germany) (Strüder et al., 1990 (80)) of $200 \times 64$ pxl $^{2}$ with $150 \mu \mathrm{m}$ pixel size, which is used as a configurable detector (Morrison, 1994 (53)). A configured detector allows images to be taken simultaneously in different modes: differential phase contrast, dark-field contrast, and bright-field contrast (Wiesemann, 2003 (96)). The results presented here were obtained by using the bright-field technique. The CCD has 64 readout channels. The readout time of one row is $28.5 \mu \mathrm{s}$, thus the total readout time for 200 rows is $5.7 \mathrm{~ms}$, which is a minimum limit for the exposure time.

Stereo experiments at the STXM required the modification of the STXM object plate to implement the detachable tilt stage, introduced by Weiß, 2000 (94), shown in figure 4.8 right. To mount the object holder to the STXM object plate, an aluminium adapter is applied. The capillary is inserted to the front end of the axle similar to the insertion described in chapter 3.1. The capillary is tilted via an axle by a stepper motor. The axle is inserted into the STXM object plate due to the small distance of both STXM vacuum vessels for X-ray operation. The insertion causes a difference in z-position between the stepper motor and the axle, resolved by a spring. The stepper motor has an accuracy of better than $0.1^{\circ}$.

Since 2008, a compact digital camera (uEye from IDS Imaging Development Systems GmbH, Germany) with USB hub is implemented in the STXM setup, allowing for displaying the sight through the visible light microscope with a computer and storing the image. The computer display provides a higher resolution of about $1 \mu \mathrm{m}$ when combined with the $40 \mathrm{x}$ objective of the visible light microscope. The improved prealignment secures less focusing necessary with the X-radiation, and thereby less dosage applied to the sample and less expenditure of time. Both effects are important for stereo experiments. 

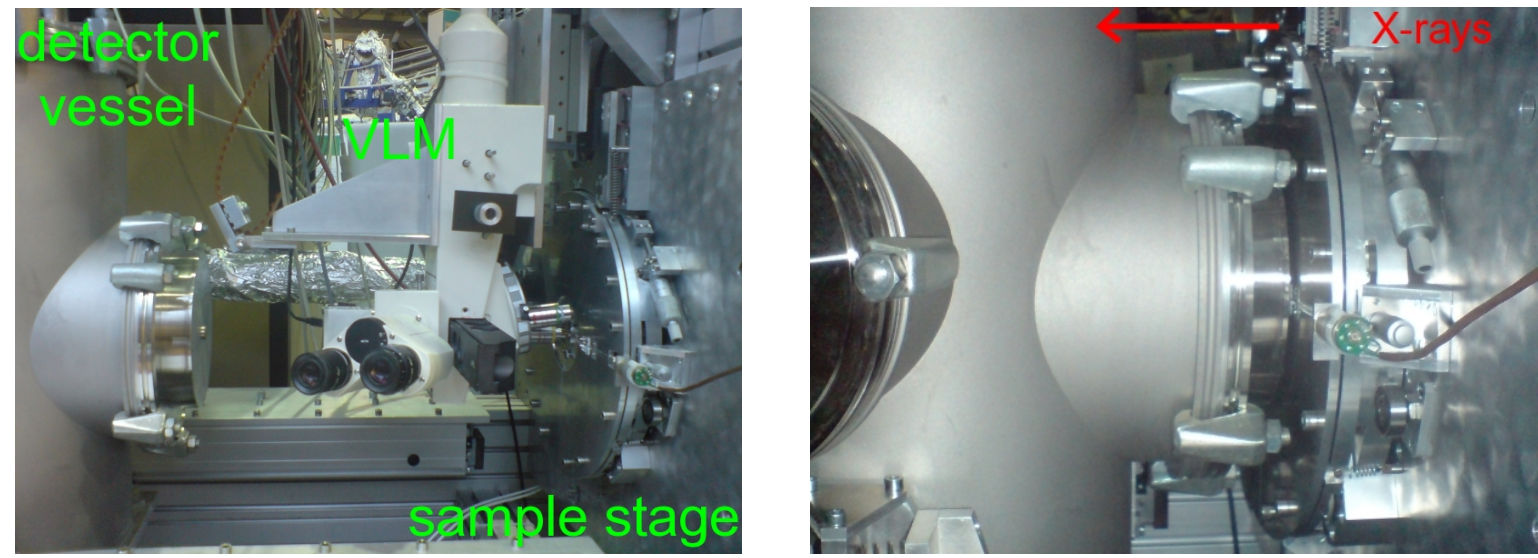

Fig. 4.7: Photographs of the STXM object stage between two vacuum vessels with insertable visible light microscope. Left: Photograph of the STXM object stage when the CCD vacuum vessel (left) is moved back from the zone plate vacuum vessel (right) and the visible light microscope is inserted. Right: Photograph of the object stage when the CCD vacuum vessel is close to the zone plate vacuum vessel, resulting in an air gap of only a few hundred micrometres for soft X-ray imaging. The green board of the stepper motor for stereo experiments is visible.
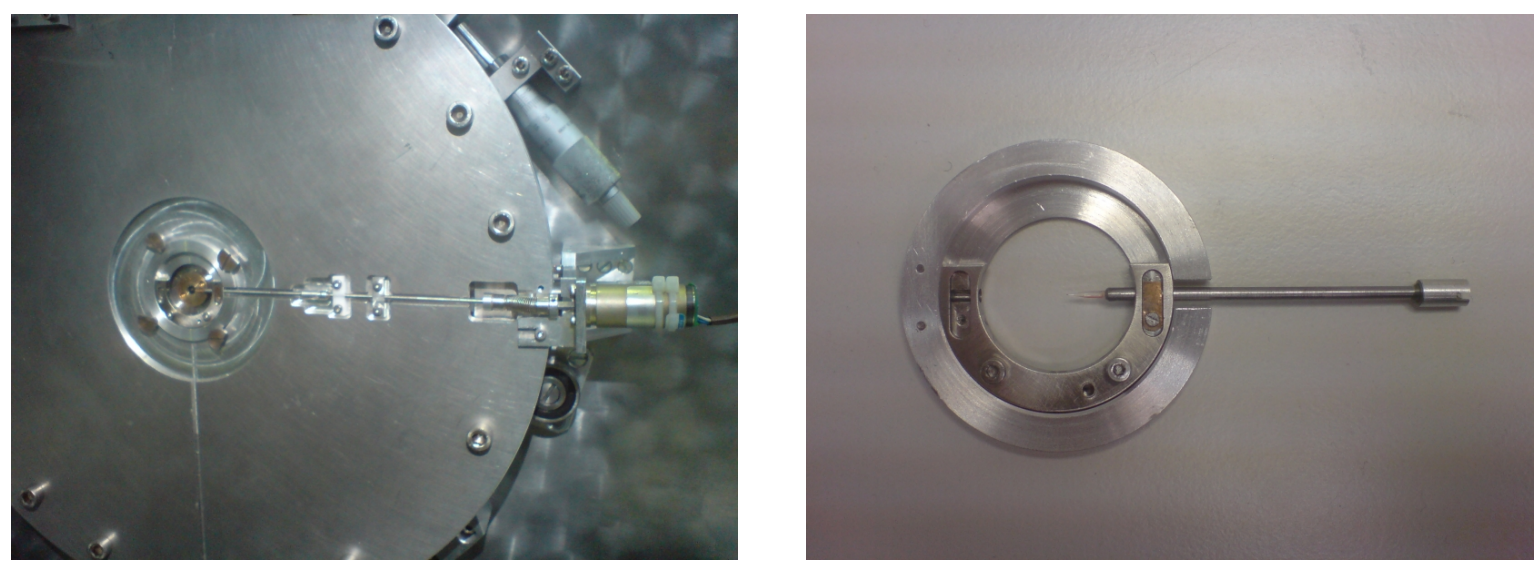

Fig. 4.8: Photographs of the STXM object holder plate modified for stereo imaging and of the detachable object holder. Left: Photograph of the STXM object holder plate modified for stereo imaging. The detachable tilt stage is mounted via an adapter (shown right besides) in front of the vacuum window of the zone plate vacuum vessel. The axle is inserted in STXM object plate to connect the tiltable sample holder with a stepper motor divided in two parts for easy object holder demounting. Right: Photograph of the detachable object holder for stereo imaging with the STXM with a capillary inserted. The detachable object holder is fixed onto an adapter for mounting to the object plate. The front-end of the divided axle is inserted. 
Furthermore, the possibility to store the prealignment images of the visible light microscope is very helpfull to recognise the initially imaged sample region after tilting the object holder. As the capillary tips are not perfectly straight, a tilt around the idealised capillary axis, considered as the $\mathrm{x}$-direction, causes a drift in $\mathrm{y}$ - and z-direction. Thus, after tilting the sample has to be brought back to the initial position in y-direction, and the focus has to be adjusted.

Also with regard to investigations of dynamics, the digital camera at the visible light microscope is important. The higher resolution enables to see movements of small samples like haematite particles which were not visible just with the visible light microscope. Thus, the success of injecting additional small particles to a sample within a capillary tip can be controlled, and sample motion too fast for detection with the STXM is identified.

The minimum distance of the capillary from the STXM zone plate is limited by the minimum distance necessary between the capillary and the vacuum window of the zone plate vacuum vessel. The tapered part of capillary has to be quite short for stability reasons (chapter 3.1). However, the vacuum window of the STXM is not on top of a cone, but of a cylindrical device of about $1.5 \mathrm{~cm}$ diameter (figure 4.8 left). Thus, attaching of the thicker part of the capillary to the cylindrical mount of the vacuum window limits the achievable distance of the tapered capillary tip from the vacuum window. On the other hand, the minimum distance between the vacuum window and the zone plate is limited due to the limited possible drive of the zone plate in z-direction. Therefore, the capillary tip could not be focused at energies below $300 \mathrm{eV}$ yet. This has to be improved in order to realise carbon mapping in combination with stereo microscopy.

First stereo experiments with the STXM can be found in Gleber et al., 2007 (23), Gleber et al., 2009 (21) and in chapter 6. For the presented experiments, a zone plate with an outermost zone width of $50 \mathrm{~nm}$ has been used.

\subsubsection{TWINMIC at ELETTRA}

The TwinMic at ELETTRA, Trieste, Italy, is a combined microscope working either in a full-field transmission mode, or in a scanning transmission mode (Kaulich et al., 2006 (37)). Both modes are performed with zone plate optics similar to the setups described above for the full-field microscopes and the scanning microscope, respectively. When working in the scanning mode, a micro zone plate focuses the X-ray light onto the sample. When switching to the full-field mode, this zone plate is exchanged automatically through a condenser zone plate and a micro zone plate is positioned between the sample 


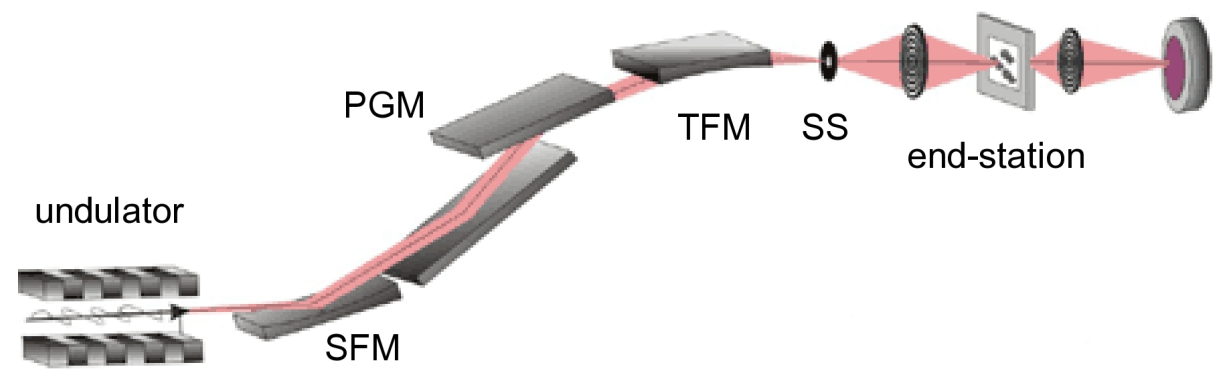

Fig. 4.9: Scheme of the $1.1 \mathrm{~L}$ beamline at ELETTRA with an undulator source, a sagittally focusing mirror (SFM), a plane grating monochromator (PGM), a toroidal focusing mirror (TFM), a secondary source (SS), and the TwinMic end-station. [Adapted from Kaulich et al., 2006 (37)]

and the detector. The illumination is adapted from coherent for the scanning mode to partially coherent for the full-field mode by dedicated condenser optics.

For the first development period the TwinMic was situated at the BACH beamline at ELETTRA. Since 2007, it is built up at the undulator U 5.6 at the beamline BL 1.1.. A scheme of the beamline layout is shown in figure 4.9 with the optical components sagittally focusing mirror, plane grating monochromator, toroidal focusing mirror, and secondary source. The short undulator is specially designed to provide a higher angular divergence of the X-ray beam. It provides highly brilliant X-ray light within an energy range of about 250 to $800 \mathrm{eV}$ in the first harmonic and about 700 to $2000 \mathrm{eV}$ in the third harmonic (www.elettra.trieste.it/experiments/beamlines/twinmic/optical_scheme.html).

The nominative monochromaticity provided by the plane grating monochromator lies in the range from $\lambda / \Delta \lambda \approx 4000-12000$. When high flux is required as for full-field imaging with short exposure times, the monochromaticity can be reduced by enlarging the slit. In continuation to the monochromator, the X-rays are focused by a toroidal focusing mirror into a pinhole, which can be chosen from diameters of 5 to $50 \mu \mathrm{m}$, forming a secondary source in front of the TwinMic end-station. The sample stage is situated in a separate module allowing for either vacuum environment, inert gas atmosphere, or ambient conditions. The detector for energies of up to $800 \mathrm{eV}$ is a thinned, back-illuminated CCD camera (Princeton) with $1340 \times 1300 \mathrm{pxl}^{2}$ with $20 \mu \mathrm{m}$ pixel size for high-lateral resolution full-field imaging. When using higher energies and for the scanning mode, an inserted phosphor screen converts the signal into visible light, which is detected by a fast read-out CCD camera (Andor). 


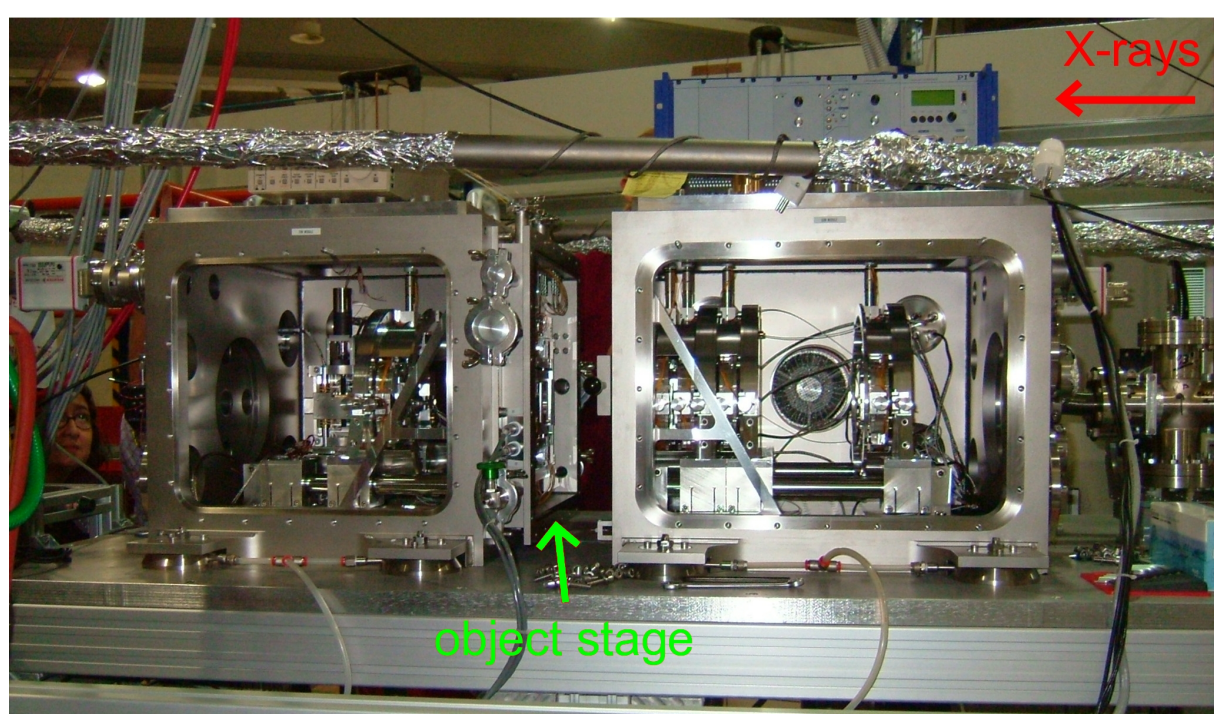

Fig. 4.10: Fotograph of the TwinMic (at the BACH beamline, hosting the TwinMic during the first development period). Both vacuum chambers are opened. The right one hosts the condenser zone plate for the full-field mode and the micro zone plate for the scanning mode. The left chamber contains the micro zone plate for the full-field mode. It is moved back giving access to the sample stage.

At the time the here presented experiments were performed, just the scanning mode was available. Although in principle possible, the stereo mount could not be inserted to the TwinMic due to mechanical problems. Furthermore, the sample had to be in vacuum for the presented experiments, resulting in a quite long time lag between preparing the sample and imaging. Due to the vacuum environment, the investigation of aqueous samples was not possible with the sample holders presented before (chapter 3.1), but requires vacuum compatible wet chambers as proposed for example by Stollberg et al., 2007 (79). In-situ sample manipulation could not be performed with that set-up. 


\section{Chapter 5}

\section{Samples from environmental science}

The paedosphere (Greek: pedon-ground) incorporates the area, where the lithosphere, which denotes the outer solid mantle of the earth, is interpenetrated together by the hydrosphere, the atmosphere, and the biosphere. Thus, within the paedosphere changes of the components of the lithosphere are influenced by water, air and biota at the same time. Within the paedosphere under the presence of all four spheres mentioned, soil formation (paedogenesis) occurs. The reaction product from influences and interactions of water, air, and biota with rock forms soil, thus resulting in the four major soil constituents inorganic matter, organic matter, water, and air. The inorganic soil fraction is formed by soil minerals originating from weathering of sedimentary rocks. The soil minerals mostly consist of the elements oxygen, silicon, aluminium, iron, calcium, magnesium, sodium, and carbon, and occur either as silicates or oxides (Tan, 2000 (83)). The organic fraction of soils is constituted by dead organic matter. The dead organic matter exists nonhumified as original or slightly modified animal, plant, or microbial tissue, and humified products from the synthesis of nonhumified matter. The soil biota are not included in the organic soil fraction.

The heterogeneous composition of soils underlies the history of the soil formation as well as various environmental impact factors and shows a great variety all over the world. Hence, geological, biological, chemical and physical interrelations are quite complex and object of ongoing research with a high demand for interdisciplinarity. One still open question is about the functionality and control of transport, bond, and bioavailability of matter within soils (e.g. Schulz and Hedeler, 2003 (69)). The high relevance of this issue becomes clear when it refers to the release and transport of materials anthropogenically induced to the environment (Dean, 2007 (15)). As already known, the aquatic colloids 
play a major role for these processes (Tan, 2000 (83)), but their behaviour is still poorly understood (Hofmann et al, 2003 (30)).

\subsection{Inorganic soil fraction}

The inorganic soil fraction is characterised as conglomeration of different grain sizes. The classification is based on a logarithmic scale formulated by the Swedish engineer Karl Atterberg in 1908. The extension of the Atterberg scale is not internationally uniform, as demonstrated in table 5.1. In Germany, the size separators are given by the German industry norm DIN 4022 to grain diameters of $2 \cdot 10^{x}$ and $6.32 \cdot 10^{x} \mathrm{~mm}$. Grain sizes above $2 \mathrm{~mm}$ are named coarse soil or soil skeleton. Grain sizes below $2 \mathrm{~mm}$ form the fine soil. According to the German sizing system, sand covers the particle size range from $2 \mathrm{~mm}$ to $63 \mu \mathrm{m}$ with the subsections coarse sand $(2000-630 \mu \mathrm{m})$, medium sand $(630-200 \mu \mathrm{m})$, and fine sand $(200-63 \mu \mathrm{m})$. Silt denotes particles with diameters between $63 \mu \mathrm{m}$ and $2 \mu \mathrm{m}$ with the subsections coarse silt $(63-20 \mu \mathrm{m})$, medium silt $(20-6.3 \mu \mathrm{m})$, and fine silt (6.3-2 $\mu \mathrm{m})$. Particles smaller than $2 \mu \mathrm{m}$ classify clay with the subsections coarse clay $(2-0.63 \mu \mathrm{m})$, medium clay $(0.63-0.2 \mu \mathrm{m})$, and fine clay $(<0.2 \mu \mathrm{m})$. The name clay is a bit irritating, as the clay fraction consists not only of clay particles, but also particles fulfilling the size criterion. The sizing system of the United States Department of Agriculture (USDA) as well as the international sizing system vary slightly from German

\begin{tabular}{rrrrr}
\hline Description & & Germany $[\mu \mathrm{m}]$ & USDA $[\mu \mathrm{m}]$ & International $[\mu \mathrm{m}]$ \\
\hline \multirow{3}{*}{ Sand } & very coarse sand & - & $2000-1000$ & - \\
& coarse sand & $2000-630$ & $1000-500$ & $2000-200$ \\
& medium sand & $630-200$ & $500-250$ & - \\
& fine sand & $200-63$ & $250-100$ & $200-20$ \\
& very fine sand & - & $100-50$ & - \\
\hline \multirow{3}{*}{ Silt } & coarse silt & $63-20$ & $50-20$ & $20-2$ \\
& (medium) silt & $20-6.3$ & - & - \\
\hline \multirow{3}{*}{ Clay } & fine silt & $6.3-2$ & $20-2$ & - \\
& coarse clay & $2-0.63$ & - & - \\
& $($ medium) clay & $0.63-0.2$ & $<0.2$ & - \\
\hline
\end{tabular}

Table 5.1: Grain sizing of fine soil particles according to the German, the USDA, and the international classification system. 
one. The main differences is the separation from sand and silt, which is made at $50 \mu \mathrm{m}$ in the USDA classification system and at $20 \mu \mathrm{m}$ in the international classification system (Soil Survey Division Staff, 1993 (72)). The soil taxonomy for mixed unconsolidated sediments, as composites of different percentages of sand, silt, and clay, is given in detail by Soil Survey Staff, 1999 (73).

Sediments form approximately $8 \%$ of the lithosphere, but cover approximately $75 \%$ of the earth's surface (Scheffer et al, 1992 (17)). The mineral composition of the pedosphere is the subject of soil mineralogy. The dominant mineral is quartz with a percentage of approximately $40 \%$ of the total weight, followed by carbonates $(20 \%)$ and mica $(20 \%)$, clay minerals (9\%) and feldspars (7\%) (Ahl et al, $2007(1)$ ). Here, quartz, mica and feldspar belong to the primary minerals. They have been released from rocks and are chemically unchanged. In contrast, the secondary minerals like clay minerals and carbonates originate from weathering on primary minerals and may show chemically changed conditions. According to the grain sizing, primary minerals are more abundant within the sand fraction, while secondary minerals are mainly present in the clay fraction. However, this allocation is not rigid, e.g. quartz can also appear as secondary mineral in the clay fraction.

Referring to the constituents of the clay fraction, clays can be divided in amorphous clays, crystalline silicate clays, sesquioxide clays and silica minerals (Tan, 2000 (83)). The major minerals of the silica minerals are quartz, $\mathrm{n}\left(\mathrm{SiO}_{2}\right)$ and crystobalite, $\mathrm{n}\left(\mathrm{SiO}_{2}\right)$. Sesquioxide clays are based on goethite, $\alpha-\mathrm{FeO}(\mathrm{OH})$, haematite, $\alpha-\mathrm{Fe}_{2} \mathrm{O}_{3}$, and gibbsite, $\mathrm{Al}(\mathrm{OH})_{3}$ (section 5.1.2). Crystalline silicate clays are formed by phyllosilicate minerals, combining various kinds of sheet silicates.

The characteristic structural elements of the phyllosilicates are the tetrahedral and octahedral sheets. Schemes of the structure of a two-layer and a three-layer phyllosilicate mineral and the respective lattices are shown in figure 5.1, adapted from Lagaly and Köster, 1993 (44). The two-layer clay minerals are built from one tetrahedral sheet (T) and one octahedral sheet $(\mathrm{O})$, thus called 1:1 sheet silicates (figure 5.1 top). The corresponding layer distance $d_{\mathrm{L}}$ is around $0.7 \mathrm{~nm}$ to $0.73 \mathrm{~nm}$. The three-layer clay minerals are built from two outer tetrahedral sheets and one octahedral sheet in between, called 2:1 sheet silicates (figure 5.1 bottom). The assembly of 2:1 sheet silicates varies and shows in most cases surface charges as demonstrated in the schematic. The corresponding layer distance $d_{\mathrm{L}}$ is around $0.7 \mathrm{~nm}$ for $1: 1$ sheet minerals, and about $1 \mathrm{~nm}$ for 2:1 sheet minerals if no water molecules are bond in between. An example for a 1:1 sheet silicates is kaolinite. Nontronite and montmorillonite are 2:1 sheet silicates. 


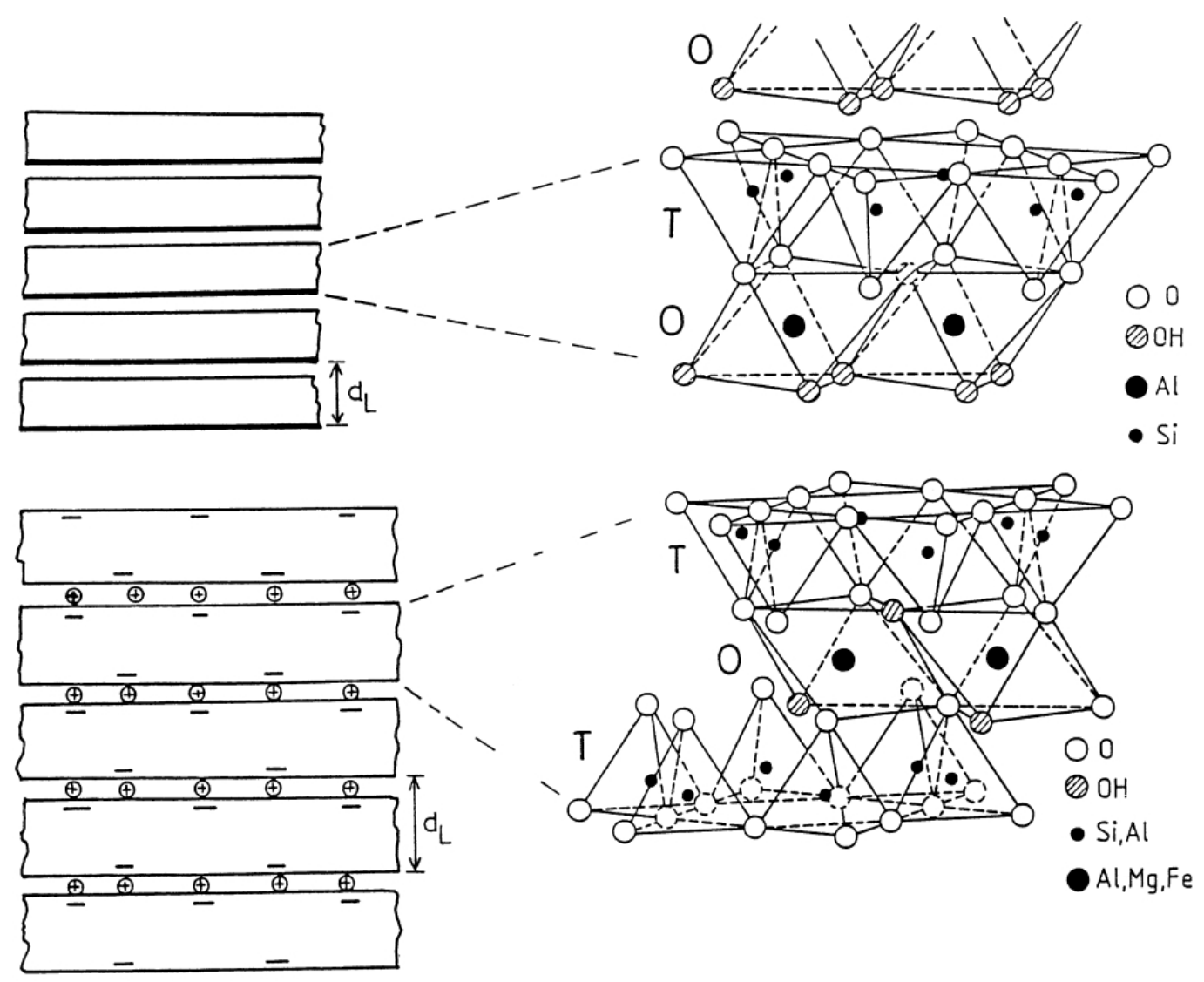

Fig. 5.1: Structures of two-layer clay minerals (top) and three-layer clay minerals (bottom) and the respective lattices. Two-layer clays mineral are made up of one tetrahedral sheet (T) and one octahedral sheet $(O)$, thus called 1:1 sheet silicates. The corresponding layer distance $d_{\mathrm{L}}$ is around $0.7-0.73 \mathrm{~nm}$. Three-layer clay minerals are made up of two tetrahedral sheets and one octahedral sheet, called 2:1 sheet silicates. The assembly of 2:1 sheet silicates varies and shows in most cases surface charges as demonstrated (bottom). The corresponding layer distance $d_{\mathrm{L}}$ is around $1 \mathrm{~nm}$ if no water molecules are bond in between. [From Lagaly and Köster, 1993 (44)]

The tetrahedral sheet is formed by $\mathrm{SiO}_{4}$ tetrahedra, resulting in a 2:5 ratio of silicon to oxygen. The $\mathrm{Si}^{4+}$ ion can be partially substituted by an $\mathrm{Al}^{3+}$ ion. The octahedral sheet consists in $\mathrm{AlO}_{6}$ octahedra. All oxide ions of the octahedra, which are not also a part of a tetrahedron, are bound with a proton forming a hydroxide ion. Minerals with all positions within the oxide ion octahedra being occupied are called trioctahedral. Dioctahedral minerals have vacancies in about a third of the octahedra positions. In the octahedra, the aluminium ions are for some clays substituted by manganese or iron ions. The substitution with metall ions of less valency causes a charge of the sheet. The sheet charge $\xi$ is measured as charge per unit defined by the chemical formula. The 


\begin{tabular}{cll}
\hline \multicolumn{1}{c}{ Type } & Group & Example \\
\hline 1:1 sheet mineral & Serpentine-kaolin group $(\xi \approx 0)$ & Kaolinite $($ di $)$ \\
\hline $2: 1$ sheet mineral & Talc-pyrophyllite group $(\xi \approx 0)$ & \\
& Smectites $(\xi \approx 0.2-0.6)$ & Saponite $($ di $)$ \\
& & Montmorillonite (tri) \\
& Vermiculites $(\xi \approx 0.6-0.9)$ & \\
& Illites $(\xi \approx 0.6-0.9)$ & Pontronite (tri) \\
& Micas $(\xi \approx 1)$ & Muscovite $($ di $)$ \\
& & Margarite $($ di $)$ \\
\hline 2:1 sheet mineral & Chlorite $(\xi$ variable $)$ & \\
\hline
\end{tabular}

Table 5.2: Classification of sheet silicates by sheet charge $\xi$. [Adapted from Lagaly and Köster, 1993 (44) (without subgroups)]

classification of the sheet silicates by sheet charge is presented in table 5.1, adapted from Lagaly and Köster, 1993 (44) (without subgroups).

To neutralise the sheet charge, cations are bound between the particular sheets, and water molecules are embedded. The layer distance increases stepwise in hydration levels depended on the amount of embedded layers of water molecules (Lagaly, 1993 (43)). With increasing partial pressure of water vapour, or decreasing electrolyte concentration, the layer distance increases from about $1 \mathrm{~nm}\left(0^{\text {th }}\right.$ hydration level $)$, where no complete, monomolecular water layer exists between the mineral sheets, to distances of up to $2 \mathrm{~nm}$, where four water layers are situated between the mineral sheets $\left(4^{\text {th }}\right.$ hydration level). At this state, referred to as quasi-crystalline, the crystalline order of the clay structure is still widely existent. Montmorillonite for example holds a water content of over $40 \%$ still remaining in the quasi-chrystalline state. In water, 2:1 sheet minerals with a sheet charge $\xi$ smaller than 0.55 charges per formula unit can increase the layer distance even further than $2 \mathrm{~nm}$, while the 1:1 sheet minerals do not show any swelling. This swelling means a separation of the sheets, resulting in a colloidal dispersion of separated silicate sheets or thin stacks of several silicate sheets. In the case of sodium smectite, the inter layer cations are then forming diffuse ion layers in the water around the dispersed silicate sheets, referred to as Gouy-Chapman-layer. Under these conditions, the dispersed particles underlie the colloidal interactions described by the DLVO theory (chapter 5.3.1). 
The inclusion of cations between the mineral sheets is reversible. Thus, with a high amount of inter layer cations, and due to the enormous surface of up to $800 \mathrm{~m}^{2}$ per gramme, clay minerals have a high cation exchange capacity, which is also element specific. In soils, this is of major importance for the supply of plants with nutrients. Clays, as well as humic substances (chapter 5.2), determine the cation exchange capacity of soils (e.g. Ahl et al., 2007 (1), Scheffer et al., 1992 (17), Tan, 2000 (83), and in detail and with respect to specific cycles in Stevenson and Cole, 1999 (77)). Thus, the contingent and sort of clay in soils influences strongly the soil fertility. The corresponding processes occur in a time range of several minutes, dependent on the swelling state of the clay mineral or rather the thickness of water layer between the clay sheets.

Further details about clay minerals can be found for example in Jasmund and Lagaly, 1993 (36) and Bergaya et al., 2006 (6).

\subsubsection{Examples for clay minerals}

Three common clay minerals, which are chosen as clay samples for the experiments presented in chapter 7 , are introduced in the following.

Among the 1:1 sheet phyllosilicate, kaolinite is one of the most common. As kaolin, it is mined all over the world. The chemical composition for kaolinite is $\mathrm{Al}_{2} \mathrm{Si}_{2} \mathrm{O}_{5}(\mathrm{OH})_{4}$. The name is derived from the kaolinite-rich Gaoling or Kao-Ling ("High Hill") in Jingdezhen, Jiangxi province, China. As dioctahedral 1:1 sheet silicate, kaolinite has a low shrink-swell capacity and a low cation exchange capacity (1-15 meq/100g.). It is a soft, earthy, usually white clay, produced by the chemical weathering of aluminium silicate minerals like feldspar.

Montmorillonite is a trioctahedral 2:1 sheet silicate. Its water content is variable and it increases greatly in volume when it absorbs water. The chemical composition for montmorillonite is $(\mathrm{Na}, \mathrm{Ca})_{0.33}(\mathrm{Al}, \mathrm{Mg})_{2}\left(\mathrm{Si}_{4} \mathrm{O}_{10}\right)(\mathrm{OH})_{2} * \mathrm{n} * \mathrm{H}_{2} \mathrm{O}$. Potassium, iron, and other cations are common substitutes. Montmorillonite is a very soft phyllosilicate mineral that typically forms in microscopic length scales crystals. Montmorillonite is a member of the smectite family with a high swelling capacity and a high sheet charge $(\xi \approx 0.3-0.4)$ and thus a high cation exchange capacity. The particles are plate-shaped with an average diameter of approximately $1 \mu \mathrm{m}$. It is named after Montmorillon in France.

Another example for a trioctahedral 2:1 sheet silicate is nontronite. Nontronite is the iron(III) rich member of the smectite group of clay minerals. Nontronites typically have a chemical composition consisting of more than about $30 \% \mathrm{Fe}_{2} \mathrm{O}_{3}$ and less than about $12 \%$ 
$\mathrm{Al}_{2} \mathrm{O}_{3}$. Like montmorillonite, nontronite can have variable amounts of adsorbed water associated with the interlayer surfaces and the exchange cations. A typical structural formula for nontronite is $\mathrm{Ca}_{.5}\left(\mathrm{Si}_{7} \mathrm{Al}_{.8} \mathrm{Fe}_{.2}\right)\left(\mathrm{Fe}_{3.5} \mathrm{Al}_{.4} \mathrm{Mg}_{.1}\right) \mathrm{O}_{20}(\mathrm{OH})_{4}$. The dioctahedral sheet of nontronite is composed mainly of trivalent iron $\left(\mathrm{Fe}^{3+}\right)$ cations, although some substitution by trivalent aluminium $\left(\mathrm{Al}^{3+}\right)$ and divalent magnesium $\left(\mathrm{Mg}^{2+}\right)$ does occur. The tetrahedral sheet is composed mainly of silicon $\left(\mathrm{Si}^{4+}\right)$, but in about 1 from 8 cases it can be substituted by either $\mathrm{Fe}^{3+}$ or $\mathrm{Al}^{3+}$, or combinations of these two cations. Thus, nontronite is characterised by having most (usually greater than 60\%) of the layer charge located in the tetrahedral sheet. The layer charge is typically balanced by divalent calcium $\left(\mathrm{Ca}^{2+}\right)$ or magnesium $\left(\mathrm{Mg}^{2+}\right)$.

\subsubsection{Iron oxides}

Among other cations, iron oxides are ubiquitous in seepages and groundwaters. They impact significantly many properties of and processes in the soil systems. In colloidal forms, iron oxides appear abundantly in soils and aquifers as goethite, ferrihydrite, haematite, and magnetite. They regulate the concentration of nutrients and pollutants and function as electron donator or acceptor in redox-reactions of microbial metabolism (Weber et al. 2006 (93)).

In total, there are 16 iron oxides, referring to oxides, hydroxides and oxide-hydroxides, in detail described by Cornell and Schwertmann 2003 (14). They are composed of iron, oxygen and/or hydroxide. The basic unit structure of all $\mathrm{Fe}^{\mathrm{III}}$ oxides consists in an octahedron, where each Fe atom is surrounded by either six $\mathrm{O}$ ions, or by both $\mathrm{O}$ and $\mathrm{OH}$ ions. These surrounding ions form either approximately hexagonally closed-packed layers (as in the case of haematite and goethite, see below), or approximately cubic closepacked layers. The characterisation and the preparation of iron oxides is explained by Schwertmann and Cornell 2000 (70).

Iron oxides occur bivalent or trivalent, depending on the redox condition. Ferrous iron, $\mathrm{Fe}^{2+}$, is abundant under anaerobic conditions but instable under aerobic conditions. At the groundwater surface or by mixing of anaerobic bankfiltrate and aerobic water the groundwater may get in contact with oxygen. Ferrous iron will be oxidised to ferric iron, $\mathrm{Fe}^{3+}$, a source for precipitation. It gives rise to the formation of new colloidal particles (Hofmann et al. 2003 (30)).

The two most abundant colloidal iron oxides in soils are haematite and goethite, followed by the poorly crystallised hydrous ferrihydrite (Sparks et al. 1995 (74)). Haematite $\left(\mathrm{Fe}_{2} \mathrm{O}_{3}\right)$ has an intense red colour and usually a crystal shape of hexago- 
nal platelets or rhombohedra. The density of haematite is in the range of $5.12 \mathrm{~g} / \mathrm{cm}^{3}$ to $5.3 \mathrm{~g} / \mathrm{cm}^{3}$. Goethite $(\alpha-\mathrm{FeO}(\mathrm{OH}))$ is of a characteristic brownish-reddish yellow colour and forms needle-like and lath-like crystals. Its iron content is about up to $62 \%$, but decreases with the inclusion of crystal water. Goethite has a density of $4.3 \mathrm{~g} / \mathrm{cm}^{3}$.

The colloid fraction in soils, mainly clays, oxides, and organic substances, show significant effects on many soil chemical processes due to their high specific surface areas and especially in the micro pore system built with colloids (section 5.3). The surface of these colloids is charged, clays as well as organic substances show within a wide pHrange $(3 \leq \mathrm{pH} \leq 8)$ negative charges, whereas iron oxides are positively charged in the same range (section 5.3.2). Therefore, iron oxides are able to bind other soil particles forming a micro texture. Small iron oxide particles might penetrate the formerly loose colloidal matrix and solidify it due to electrostatic interaction. A change in the texture of the microstructure of soils, which leads to a change in the morphology of associations of soil colloids, influences for example runoff and erosion losses within soils. The relationship between iron oxide and the stability of soil colloid associations is however still debated in soil science, for example by Duiker et al. 2003 (16) or Rhoton et al. 2003 (63).

\subsection{Organic soil fraction}

The inorganic soil fraction consists in the dead organic matter, thus nonhumified as original or slightly modified animal, plant, or microbial tissue, and humified products from the synthesis of nonhumified matter. The decomposition of the inital matter is made by mechanical or microbial processes. Mechanical decomposition processes take place due to organisms by biting into pieces or eating, and by weathering. The microbial reduction processes occur as microbial anabolism and catabolism (for example Coates et al. 2000 (13)). The microbial reduction leads to microbial tissue and as a mineralisation process to anorganic matter under redundancy of plant nutrients.

Soil organic matter, also referred to as humus, includes all organic matter in soil except soil biota, undecayed tissue from plants or animals, and their partial decomposed products. It contains nonhumic substances, which are biochemical compounds such as proteins, fats, resins, cellulose, lignins, chitins, and carbohydrates, synthesised by living organisms (details for example in Ahl et al, 2007 (1)). Another part of soil organic matter are the humic substances, products of secondary synthesis reactions. Humic substances are of brown or black colour and relatively high molecular weight (Stevenson, 1994 (76)).

Humic substances are divided into three fractions. Humic acids are soluble within the basic $\mathrm{pH}$ range and precipitate in dilute acids. They are highly molecular com- 
pounds with a relatively high content of aromatic components. With higher valence cations, humic acids form sparingly soluble humates. Fulvic acids are soluble at any $\mathrm{pH}$ value. They have a relatively low molecular weight, a high content of functional groups, particularly carboxyl groups, and a low content of aromatic groups. Humins are insoluble at any $\mathrm{pH}$ value.

Humic substances form particles smaller than $2 \mu \mathrm{m}$ (Scheffer et al., 1992 (17)). The specific surface of humic substances exceeds with $800-900 \mathrm{~m}^{2}$ per gramme even the high specific surface of clay minerals. Also the cation exchange capacity of humic substances is comparable with the one of clay minerals (chapter 5.1). However, the negative charge of the humic acids is $\mathrm{pH}$ dependent and so is its cation exchange capacity. The point of zero charge (chapter 5.3.2) of humic acids is typically at a $\mathrm{pH}$ value of about 3 . With an increase of $\mathrm{pH}$ values higher than 3 , the negative charge of humic acids increases due to deprotonation or dissociation of protons to functional groups as carboxyl groups, phenolic OH groups, and enols. (Sparks, 1995 (74)).

The combination of humic substances with clay minerals leads to an aggregation of soil particles. The binding of humic substances by clay minerals is described by Lagaly, 1993 (43). It varies from bindings at the outer basal plane and between the clay sheets. The binding in 1:1 sheet silicates is due to penetration of the molecules of

the humic substances into uncharged silicate sheets. This embedding is not remaining and the organic molecules can be substituted. In the case of 2:1 sheet silicates, further bindings occur as solvation based binding of neutral molecules in the inter layer spaces, the replacement of water embedded in the inter layer spaces, the complex formation with inter layer cations, the substitution of inter layer cations by organic cations, the embedding of organic molecules after the previously mentioned cation substitution, and binding of organic molecules in the inter layer space by redox reactions.

Comprehensive literature about soil science and interaction of organic and anorganic soil constituents can be found for example in Scheffer et al. 1992 (17), Sparks et al. 1995 (74), and Tan, 2000 (83).

\subsection{Soil colloids}

Colloids (Greek: kollo-glue; eidos - form, appearance) are defined as particles with sizes from $1 \mathrm{~nm}$ to $1 \mu \mathrm{m}$ in at least one characteristic dimension. Due to the huge surface to volume ratio, the surface characteristics are very important. Indeed, the behaviour of colloidal matter differs significantly from the same matter as bulk. 
As seen in the previous sections, soil constituents delaminated in aqueous media are in the size range of colloids and form colloidal systems. As such, the absorption properties change towards an increased soil compaction. In colloidal form or colloidally bounded, the bioavailability of matter changes when the activity of colloidally bounded matter is reduced. Colloids have a strong influence on the matter transport within soil layers. They act also as matter carriers, where colloidally bounded matter differs much faster through the soil layers than predicted according to the classical two-phases system, which is based on seepage water dissolved matter and soil matrix bounded matter. The resulting underestimation of matter transport is of high significance in cases of pollutants like radio nuclides and heavy metals.

Details about colloid science are given by Brezesinski and Mögel, 1993 (10).

\subsubsection{Interactions of colloids}

In the above introduced case of a dispersed mineral sheet with negative sheet charge (chapter 5.1), the cations from the interlayer in the former crystalline state surround the single sheets or sheet stacks as a diffuse counter ion cloud in a so-called Gouy-Chapmanlayer (Lagaly, 1993 (43)). The concentration of the cations decays exponentially away from the negatively charged sheet surface, referred to as Stern-layer. Thereby, anions are suppressed towards the solution. With increasing temperature, cations leave the Stern-layer and form the Gouy-Chapman layer. But specific bindings like by cationic tensides, as well as a high electrolyte concentration within the dispersion, improve the occupation of the Stern-layer. The extension of the Gouy-Chapman-layer is diminished by an increasing electrolyte concentration.

In the Gouy-Chapman model of a diffuse ion cloud, the ion density $c_{i}$ in a distance $r_{s}$ from the surface is given by

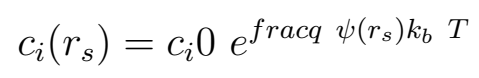

with the ion charge $q$, the electrostatic potential $\psi\left(r_{s}\right)$, the Boltzmann constant $k_{B}$, and the temperature $T$ (Lagaly et al., 1997 (45)). The electrostatic potential is derived from the Poisson equation

$$
\Delta \psi=-\frac{\rho}{\varepsilon_{r} \varepsilon_{0}}
$$

with the dielectric constants $\varepsilon_{0}$ of vacuum and $\varepsilon_{r}$ of the dispersion media. In the DebyeHückel approximation, the linearised form of equation 5.2 describes the potential for 
small values. The electrostatic potential for spherical particles of the diameter $a$ is then derived to

$$
\psi=\psi_{0} \frac{a}{a+r_{s} / 2} e^{-\kappa r_{s}}
$$

with the extension of the diffuse ion layer (Debye-Hückel length) $\kappa^{-1}$

$$
\kappa^{2}=\frac{1}{\varepsilon_{r} \varepsilon_{0} k_{B} T} \Sigma_{i} q_{i}^{2} c_{i} 0
$$

The potential curve decreases approximatively for spherical particles of the same size:

$$
V_{e l}\left(r_{s}\right)=\frac{a}{2} \frac{32 \pi \varepsilon_{r} \varepsilon_{0}\left(k_{b} T\right)^{2}}{q^{2}} \gamma^{2} e^{-\kappa r_{s}}
$$

with

$$
\gamma=\left(e^{-\frac{q \psi_{0}}{2 \varepsilon_{r} \varepsilon_{0} k_{B} T}}-1\right) /\left(e^{-\frac{q \psi_{0}}{2 \varepsilon_{r} \varepsilon_{0} k_{B} T}}+1\right)
$$

Thus, the electrostatic interaction between the colloidal particles is determined by the repulsive potential of the diffuse cation layers. With decreasing thickness of these layers, thus with increasing concentration of electrolytes or cation tensides, the repulsion acts at shorter distances, but with increased force. Typically, the length scales for the electrostatic potential are in the range of tens of nanometres (Lagaly et al., 1997 (45)).

Attraction between atoms is evoked by the van-der-Waals interaction. For two spherical particles with the diameter $a$ and the center-to-center distance $r$, this attraction is according to Lagaly et al., 1997 (45):

$$
V_{v d W}(r)=\frac{H_{k}}{6}\left(\frac{2}{s^{2}-4}+\frac{2}{s^{2}}+\ln \frac{s^{2}-4}{s^{2}}\right)
$$

with the distance given in units of particle radii $(s=2 r / a) . H_{k}$ is the material specific Hamaker constant.

Thus, independent from the electrolyte concentration, the van-der-Waals-interaction causes particle attraction of the sheet silicates. At long range, the $1 / d^{7}\left(1 / d^{2}\right.$ for $\left.r \gg a\right)$ dependent attracting van-der-Waals force is minor to the repulsive static energy. However, at short distances in the range of $1 \mathrm{~nm}$, the attracting energy preponderates, and the dispersed mineral sheets coagulate. The electrolyte concentration causing a counter ion cloud thin enough so the corresponding repulsive energy is just outbalanced by the attractive energy, is called critical coagulation concentration. The colloidal dispersion is stable below this critical electrolyte concentration. 
The classical DLVO model, named after its originators Derjaguin and Landau from Russia as well as Verwey and Overbeek from the Netherlands, describes the colloid stability based on the long range attractive London-Van-der-Waals forces between nonpolar atoms or molecules (equation 5.7) and electrostatic repulsive interactions (equation 5.5) resulting from surface charges of colloids (Lagaly et al., 1997 (45), Hofmann et al. $2003(30))$. Analogously to the Lennard-Jones potential, a very short range $\left(1 / r^{12}\right)$ repulsive potential is added (Vormoor, 2002 (90)).

Extensions of the DLVO theory in order to improve the model applicability on colloid stability within more complex, environmental systems can be found for example in Lagaly et al., 1997 (45) and Grasso et al. 2002 (24). They are based on integration of osmotic pressure, effects of surface roughness, steric interactions, hydration pressure, Lewis acid base interactions, hydrogenbonding, and the hydrophobic effect.

\subsubsection{Point of zero charge}

As seen above, a part of the surface charges of soil constituents show a $\mathrm{pH}$ dependence. The point of zero charge is defined as that $\mathrm{pH}$ value of the solution, at which the net charge of the colloid surfaces is zero (Cornell and Schwertmann, 2003 (14)). Beyond the point of zero charge, the net charge is positive, and above it the net charge is negative. In general, the point of zero charge is relatively high for iron and aluminium oxides, and low for silica and humic substances (Sparks, 1995 (74)). Several methods to measure the point of zero charge are described by Appel et al. 2003 (4).

The negative charge of the basal planes of the phyllosilicates is not $\mathrm{pH}$ dependent, but within an aqueous media, the edges are surrounded by a diffuse ion cloud as discussed in chapter 5.3.1. The extension and the charge of this diffuse ion layer is $\mathrm{pH}$ dependent. This influences the aggregation of clay platelets as seen in figure 5.2. In the case of an edge to basal plane constellation, the distance is relatively large at low $\mathrm{pH}$ values and cations surround the positively charged edge. Anions are situated between these ions and the negatively charged basal plane. At the point of zero charge, the edge is not charged and only some anions are between the negatively charged basal plane and the neutral edge. At higher $\mathrm{pH}$ values, the edge is also negatively charged. Anions between the edge and the basal plane strengthen this conformation and lead to aggregation. The constellation of basal plane to basal plane shows the highest stability at low $\mathrm{pH}$ values, but exists also at higher $\mathrm{pH}$, although not as closely aggregated, because of the negative charge of the edges. The edge to edge constellation is stable at $\mathrm{pH}$ values below 


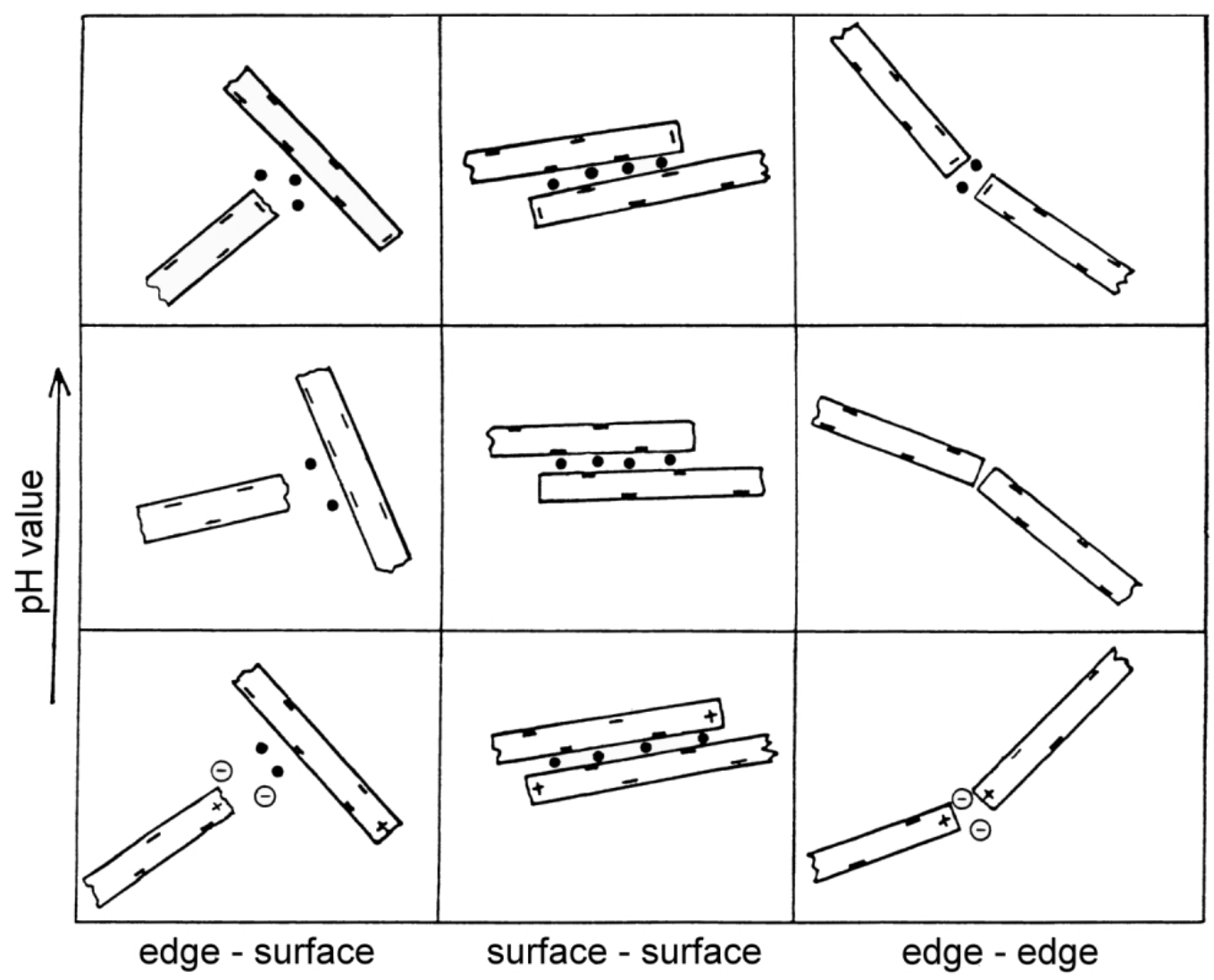

Fig. 5.2: Simplified schematic of the change of edge charges of two clay platelets due to changes in the $\mathrm{pH}$ value, and the resulting charge distribution and possible clay platelet configuration. [Adapted from Lagaly, 1993 (43)] 


\begin{tabular}{cl}
\hline Material & Point of zero charge $[\mathrm{pH}$ value] \\
\hline Kaolinit & $\approx 2.7-4.1$ (Appel et al., $2003(4))$ \\
Nontronite & $\approx 2$ (Jaisi et al., $2007(33))$ \\
Montmorillonite & $\approx 4-6$ (Sposito, $1998(75)$, Lagaly, $1993(43))$ \\
\hline Haematite & $\approx 8.5-8.8($ Cornell and Schwertmann, $2003(14))$ \\
Goethite & $\approx 9-9.4$ (Cornell and Schwertmann, $2003(14))$ \\
\hline
\end{tabular}

Table 5.3: Point of zero charge of relevant clays and iron oxides

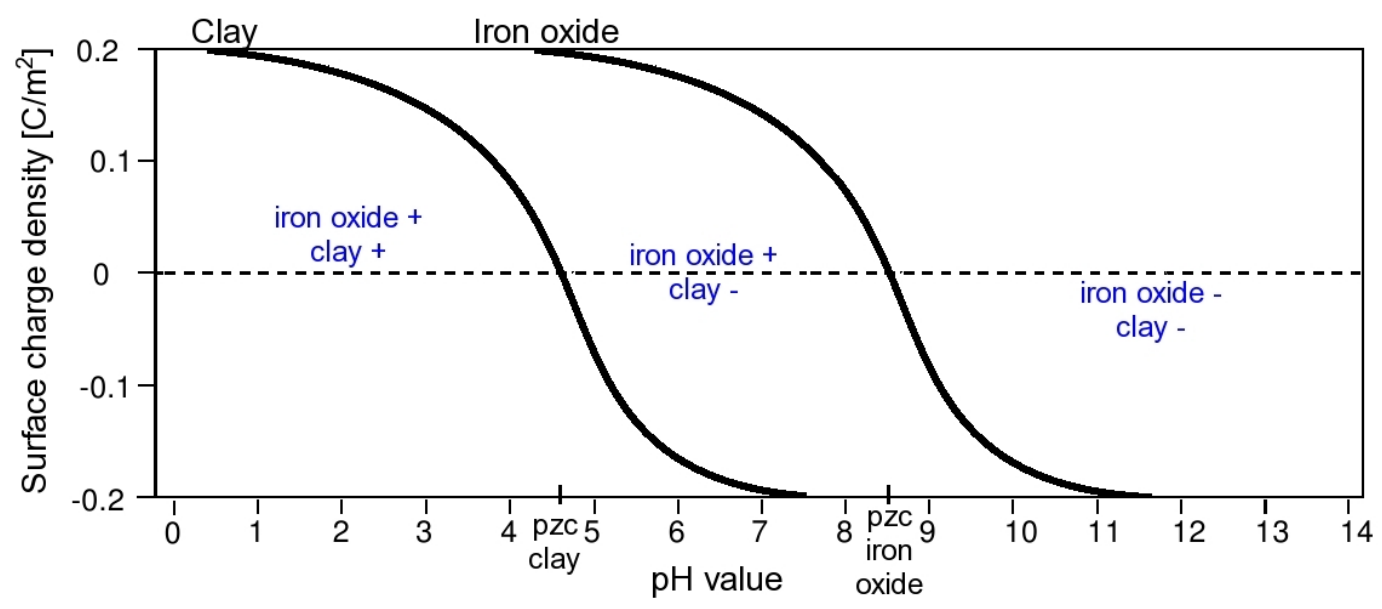

Fig. 5.3: Simplified plot of the surface charge density of a clay (montmorillonite) and an iron oxide (haematite). In the regions of low at high $\mathrm{pH}$ value, the edge surfaces of clay and iron oxide are charged equally, while they are charged contrarily at moderate $\mathrm{pH}$ values between the respective points of zero charge (pzc).

and above the point of zero charge. The equally charged edges are forced by contrarily charged ions in between. At the point of zero charge, this constellation is not stabilised.

For humic substances, the $\mathrm{pH}$ dependent charge determines the cation exchange capacity. Thus, at $\mathrm{pH}$ values below or around the point of zero charge, humic substances do not contribute to the cation exchange capacity of the soil, which is then only given by the not $\mathrm{pH}$ dependent cation exchange capacity of the clay fraction. With increasing $\mathrm{pH}$ value, the cation exchange capacity of the humic substances increases and contributes the major part of the soil cationic exchange capacity at high $\mathrm{pH}$ values.

For surfaces with no specifically adsorped surface charges, the point of zero charge is equal to the isoelectric point. The isoelectric point is the $\mathrm{pH}$ value at which a particle has no net charge and therefore shows no mobility in an external electric field. 
The point of zero charge of the clays and iron oxides investigated in this work are given in table 5.3.2 with the respective citations. At moderate $\mathrm{pH}$ values, thus between the points of zero charge of clays and iron oxides, clays show a negative surface charge, while the surface of the iron oxides is positively charged. This is plotted simplified in figure 5.3. The contrary surface charge results in an electrostatic attraction between clay and iron oxide particles. A model for this $\mathrm{pH}$ dependent attraction has been developed for the case of montmorillonite and metal by Kraepiel et al., 1999 (42).

\subsection{Examples for soils}

As examples for soils, three common soils, examined in the scope of the here presented work, are introduced. The names are according to the classification system of the Food and Agriculture Organisation of the United States (FAO) (IUSS Working Group WRB, (32)). The German names are also given according to Ahl et al., 1985 (2), as the utilised samples are taken by the authors and are well-defined in the cited work. All soil samples originate from agricultured regions around Göttingen, Germany.

Calcaric phaeozem (German: Gley-Schwarzerde or Gley-Tschernosem) is a phaeozem with calcareous in a region of at least $20 \mathrm{~cm}$ to $50 \mathrm{~cm}$ below the surface. Phaeozems have a humous surface of dark colour containing less bases than chernozem. In the first metre above the soil surface, no secondary carbonates exist.

Orthic luvisol (German: Parabraunerde) shows within the soil texture specified textural differentiation. While the surface region is depleted with clay, a deeper, agric horizon contains accumulations of high activity clay. Here, the corresponding cation exchange capacity is greater than $24 \mathrm{cmol}$ per $\mathrm{kg}$ clay.

Dystric planosol (German: Pseudogley) is a backwater influenced soil with a humous surface layer on top and a compacted layer beneath the backwater layer.

\subsection{Anthropogenically induced substances}

The anthropogenical influence on soils is tremendous in most regions of the world. The induced substances lead to changes in the interaction of soil colloids and the colloidal soil morphology.

\subsubsection{Detergents}

One example for substances daily distributed to our sewage water and thus to the environment are detergents. As described in chapter 5.3.1, cationic tensides like detergents 
improve the occupation of the Stern layer at the clay surface and thereby shorten the distance between neighboring soil colloids. Above a critical concentration, the soil colloids aggregate due to replacements of initial cations by detergents (element specific cationic exchange capacity). This aggregation in correlation with different detergent concentrations have been studied with X-ray microscopy by Niemeyer et al., 1994 (57) and Thieme and Niemeyer, 1998 (87).

\subsubsection{Carbon nanotubes}

Another anthropogenical substance is the group of carbon nanotubes. The unique capabilities of these very small tubes with diameters of about $13 \mathrm{~nm}$ to $16 \mathrm{~nm}$ find promising applications in medicine as drug carrier and engineering technologies, for example improving thermal and electric conductivity of plastics or stability properties of concrete. But the interaction with environmental colloidal systems has not been studied in detail yet, and the stabilising capabilities advantageous for engineering technologies may cause unwanted stabilities in natural colloidal systems.

For the presented experiments, two different types of carbon nanotubes were used: Baytubes $\left.{ }^{(}\right)$from BAYER AG (Leverkusen, Deutschland) and pristine carbon nanotubes (referred to as rCNT) from Dr. H. Zänker, Rossendorf, Germany. Both types are not functionalised, multi-wall carbon nanotubes, where each tube consists of several graphite layers. The production of baytubes started in 2006 with 60 metric tons per year and is being extended to 200 annual metric tons (www.baytubes.com). 


\section{Chapter 6}

\section{Experimental results}

The presented experiments were performed at the full field transmission X-ray microscopes XM-1 at the ALS in Berkeley (chapter 4.1.1) and CSXM at the KTH in Stockholm (chapter 4.1.2), as well as at the scanning transmission X-ray microscopes STXM at BESSY II in Berlin (chapter 4.2.1) and the dual mode microscope TwinMic at ELETTRA in Trieste (chapter 4.2.2).

The micrographs taken at these X-ray microscopes in absorption contrast are displayed using count numbers given by the CCD electronics. The corresponding photon number can be calculated from the amount of electron-hole pairs generated by the X-radiation. For a silicone based CCD a photon energy of $3.65 \mathrm{eV}$ creates one electronhole pair. The convertion from electrons to counts follows a camera specific transfer rate. Typical values for the quantum efficiency of such CCD devices are in the range of $60 \%$. Knowing both the incident and detected intensity, the sample thickness can be calculated based on the Lambert-Beer law (equation 2.28). However, as heterogeneous samples were investigated, and as such a calculation requires detailed knowledge of all participating elements and their respective concentrations at each sample volume projected onto one image pixel, for complex and highly inhomogeneous soil samples, this is not achievable. Furthermore, only with information about the density of each element within the composed material, conclusions about particle thicknesses can be made by this. Therefore, the presented X-ray micrographs are used only qualitatively, providing two-dimensional distribution maps of the sample particles. This is the image information necessary for stereo processing.

The samples for these experiments include two iron oxides and two clay minerals, all very abundant in the environment, and three well characterised soils. The iron oxides were haematite and goethite. Kaolinite, nontronite, and montmorillonite were chosen 
as clay samples, and calcaric phaeozem, orthic luvisol, and dystric planosol as soils (chapters 5.1 and 5.4). All samples were used as $1 \%(\mathrm{w}-\mathrm{w})$ dispersions in deionized water. As can be seen in the experiments, large clusters of colloids disaggregate to small clusters with an intact nano- and microstructure. These small clusters have dimensions in the size range accessible with X-ray microscopy. The goethite sample was purchased by Sigma Aldrich, CAS number 20344-49-4. Haematite particles with approximately $150 \mathrm{~nm}$ diameter had been made according to Schwertmann and Cornell, 2000 (70) and supplied as well as montmorillonite, descending from a reservoir at Upton, Wyoming, USA, and kaolinite by Prof. Dr. J. Niemeyer, Institute for Soil Science, University of Göttingen. The nontronite sample originates from a reservoir at Hoher Hagen, Dransfeld, Germany. Two types of carbon nanotubes were studied. The carbon nanotubes purchased from BAYER are Baytubes ${ }^{\circledR}$, and the carbon nanotubes descended from Dr. H. Zänker, Institute for Radiochemistry, Forschungszentrum Rossendorf, Germany, are referred to as rCNT (chapter 5.5.2).

The actual outcome with respect to soil science ranks behind the previously necessary demonstration of the potential of stereo soft X-ray microscopy, the performance of stereo experiments at the different X-ray microscopes, and the extension of this method to the in-situ investigation of dynamics. Nevertheless, the experiments are well understood when ordered by sample system, as for example different particle shapes have to be recognised for stereo analysis. Initial findings from pristine samples are mandatory for the interpretation of a mixture. Therefore, haematite and carbon nanotubes, which are used as additives for the mixtures, are presented first. Then, the clay samples are shown, and finally the soil samples, presenting a complex system with clay and iron oxide content. First correlations between the spatial arrangements revealed with stereo experiments and the $\mathrm{pH}$ dependent electrostatic interactions are given in the last section.

Consequently, methodical achievements are found in the course of this chapter. However, a summary of the technical achievements is given afterwards in chapter 7 .

\subsection{Haematite and goethite}

As described in section 5.1.2, haematite is an abundant iron oxide in soils. Due to its particle size, it is detectable with soft X-ray microscopy. Figure 6.1 shows a micrograph of aggregations of aqueous haematite particles between two $\mathrm{Si}_{3} \mathrm{~N}_{4}$ membranes taken with the CSXM. The imaging energy was $368 \mathrm{eV}$ and the exposure time 0.85 seconds. The scale bar indicates $1 \mu \mathrm{m}$. In the micrograph, single haematite particles of measured widths from $120 \mathrm{~nm}$ till $160 \mathrm{~nm}$ are homogeneously distributed. Also aggregations of 


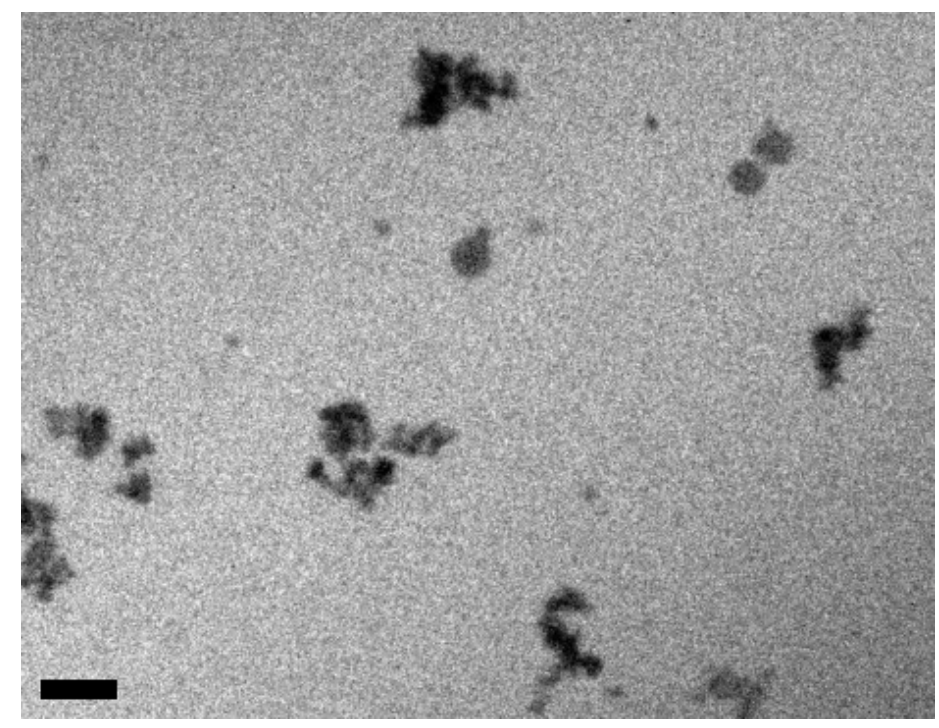

Fig. 6.1: Micrograph of aqueous haematite particles between two $\mathrm{Si}_{3} \mathrm{~N}_{4}$ membranes. The exposure time was $60 \mathrm{~s}$. Images taken with the CSXM at $E=368 \mathrm{eV}$. The scale bar indicates $1 \mu \mathrm{m}$.

haematite particles are present, with extensions of more than one micrometer. Within the aggregations, the spherical form of the single haematite particles is still visible. The spatial extension of the aggregations is demonstrated by the increase in absorption.

A dispersion of goethite, another iron oxide abundant in the environment (section 5.1.2), was filled into a capillary and imaged with the X-ray microscope XM-1. The upper image of figure 6.2 shows a cluster of particles of this goethite dispersion in a capillary of approximately $2.5 \mu \mathrm{m}$ diameter taken at $710 \mathrm{eV}$ with an exposure time of $0.85 \mathrm{~s}$ with $1024 \times 1024 \mathrm{pxl}^{2}$. In the images below, micrographs are shown taken of the same region as above and under the same imaging conditions, but after haematite particles had been added to the capillary to induce morphological changes. The tilt angle between these two images is $44^{\circ}$. When comparing the top image and the bottom image on the left side of figure 6.2, additional particles within the cluster are visible after the addition of haematite. As these particles are of different shape and smaller than goethite particles, they can be identified as haematite. Slight morphological changes of goethite clusters are also detectable, which are due to dynamics within the dispersion. The two images below were taken one hour after the addition of haematite.

In figure 6.3, details from the micrographs shown in figure 6.2 below are presented, where some edges and centres of the goethite and haematite particles have been marked. 8 structures have been marked and evaluated for their respective distances. The right 

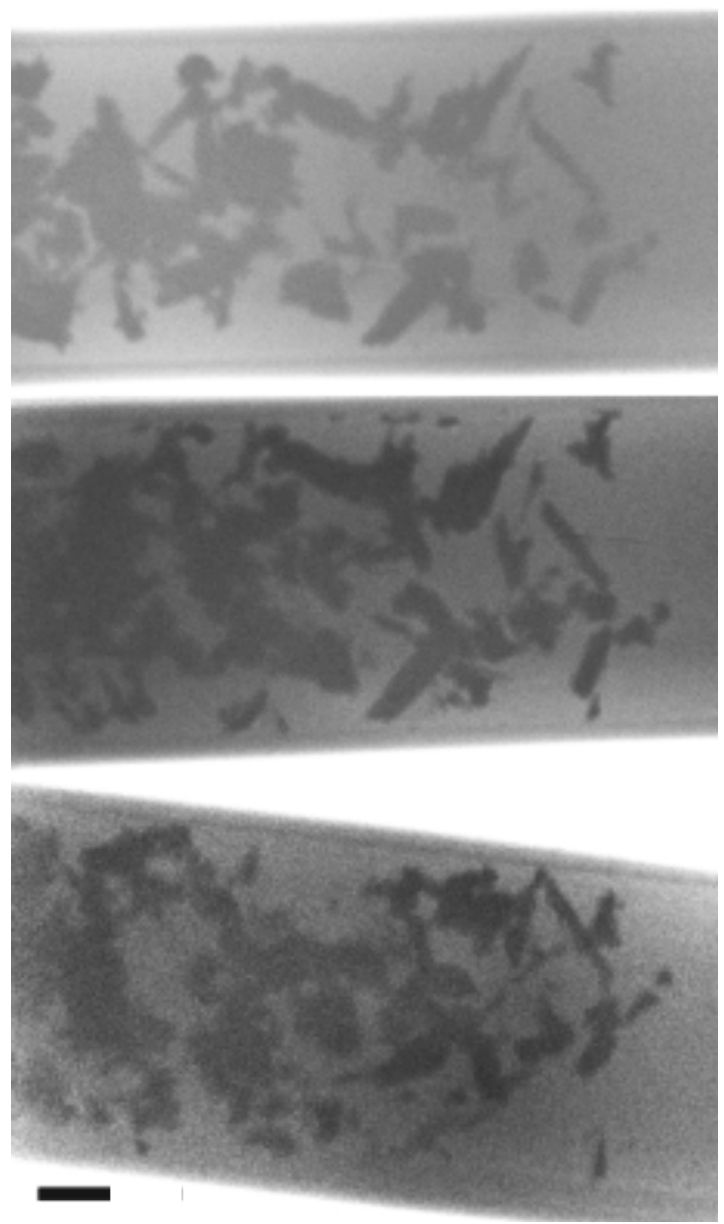

Fig. 6.2: Top: Micrograph of an aqueous goethite dispersion in a capillary of approximately $2.5 \mu \mathrm{m}$ diameter imaged at $710 \mathrm{eV}$ at the XM-1 with $0.85 \mathrm{~s}$ exposure time. Middle and bottom: Stereo micrographs of the same region as in the upper image taken with equal imaging parameters as above after adding haematite particles to the capillary. The tilt angle is $44^{\circ}$. The scale bar indicates $500 \mathrm{~nm}$. 

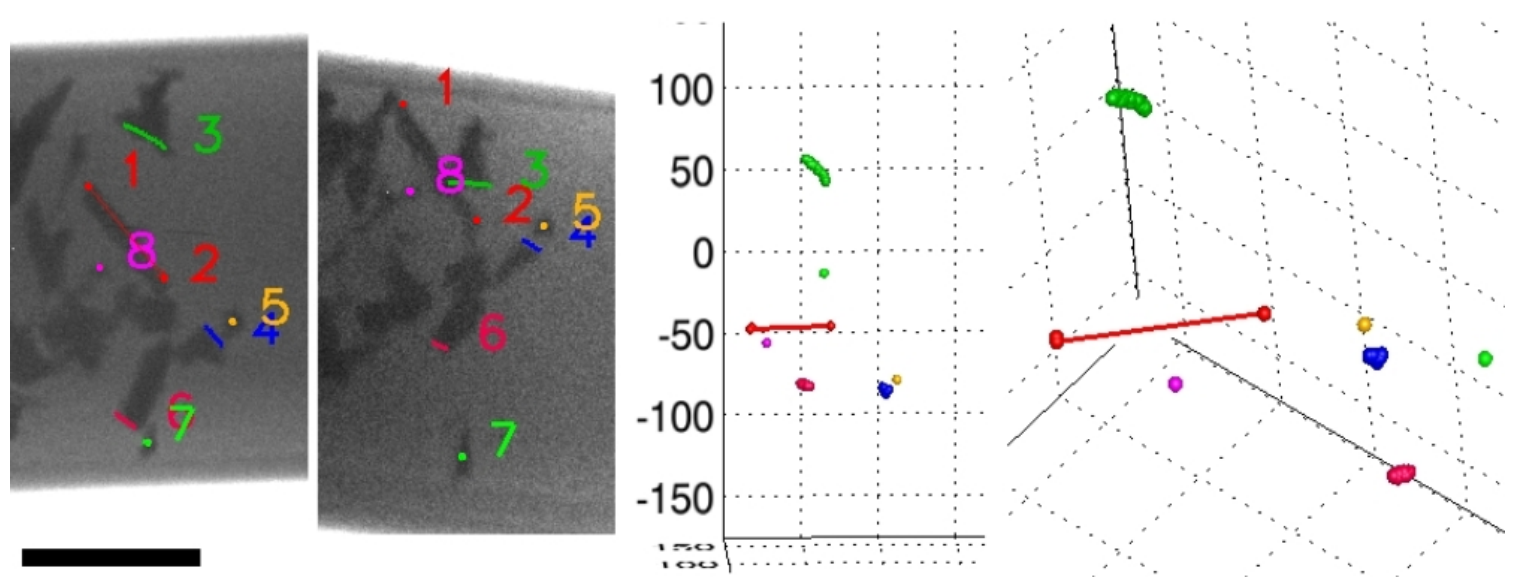

Fig. 6.3: Goethite dispersion with haematite analysed with xstereo. Left and left middle: Details from the images shown in figure 6.2 bottom. Some edges and centres of the goethite and haematite particles are marked. The scale bar indicates $500 \mathrm{~nm}$. Right middle and right: Images of three-dimensional plot of the marked structures under two different viewing angles.

plots in figure 6.3 are taken under two different viewing angles from a three dimensional plot of the marked structures, visualising the distances between the clusters and the particles. The points number 1 (red) and number 2 (red) represent two corners of a goethite particle, so the connection (red line) between both points marks the edge. When comparing these images with the top image in figure 6.2 taken before the addition of haematite, and assuming that the goethite association is spatially stable, it becomes clear that only the particles marked as number 7 and number 8 are haematite particles. To underline the potential of the presented technique, distances have been determined, e. g. the distance between the haematite particles number 8 and the edge of the goethite particle mentioned above is approximately $250 \mathrm{~nm}$.

\subsubsection{Iron mapping}

The application of elemental mapping for the detection of iron containing particles is demonstrated in figure 6.4. A montmorillonite sample is shown (more details in chapter 6.5 ), which had been mixed with haematite and let dry on a $\mathrm{Si}_{3} \mathrm{~N}_{4}$ membrane and imaged at a pre-edge energy of $704 \mathrm{eV}$ (figure 6.4 upper left), at the energy of the Fe L 3-edge $710 \mathrm{eV}$ (figure 6.4 upper right), the intermediate energy $716 \mathrm{eV}$ (figure 6.4 lower left), and the energy of the Fe L 2-edge $723 \mathrm{eV}$ (figure 6.4 lower right). The energies have been chosen according to peak position of L-3 edge of haematite (Garvie and Buseck 

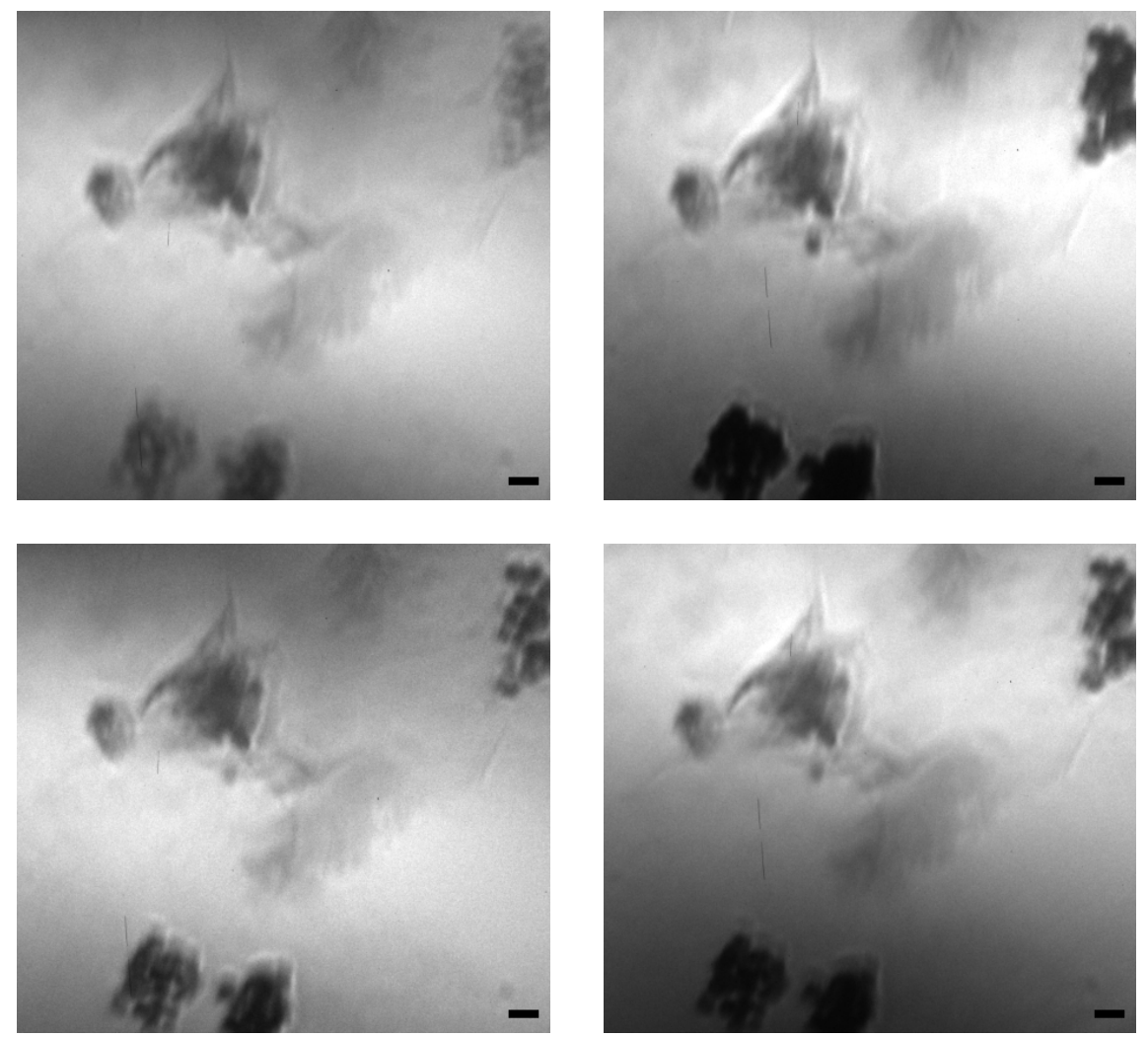

Fig. 6.4: Elemental mapping of haematite colloids within a sample of montmorillonite platelets dried on a $\mathrm{Si}_{3} \mathrm{~N}_{4}$ membrane; images taken at the pre-edge energy $704 \mathrm{eV}$ (upper left), the energy of the Fe L 3-edge $710 \mathrm{eV}$ (upper right), the intermediate energy $716 \mathrm{eV}$ (lower left), and the energy of the Fe L 2-edge $723 \mathrm{eV}$ (lower right). The scale bars indicate $1 \mu \mathrm{m}$. Images were taken with the XM-1. 
(19)). While the montmorillonite particles have the same contrast in each micrograph, the iron containing haematite particles change the contrast depending on the imaging energy. The montmorillonite cluster in the centre of the sample region contains several point-shaped structures of the same size as haematite particles. Only comparing all four images enables to distinguish between montmorillonite and haematite, as only one of these point-shaped particles shows a considerably stronger contrast in the images taken above the Fe L absorption edge. According to section 2.4.2 and the absorption spectra shown in figure 2.8, the change in contrast observed for the small particle situated at the lower edge of the centre of the cluster reveals that this is an iron containing particle: The absorption is very weak at the pre-edge energy, strongest at the Fe L 2-edge, weak at the intermediate energy and strong at the Fe L 3-edge. The same is true for the conclomerations of spherical particles in located in the upper right corner of the imaged region as well as in the area of the lower border. Thus, they can identified as hematite clusters, and the single particle with a high iron content is a single haematite particle within a montmorillonite cluster.

\subsection{Carbon nanotubes}

As an example of anthropogenically-induced material, carbon nanotubes were examined (chapter 6.2). On the left side of figure 6.5, a micrograph of a dispersion of carbon nanotubes from BAYER (baytubes) between two $\mathrm{Si}_{3} \mathrm{~N}_{4}$ membranes taken with the CSXM at $368 \mathrm{eV}$ with an exposure time of $360 \mathrm{~s}$ is presented. The scale bar indicates $1 \mu \mathrm{m}$. Over the wide sample area imaged, the aqueous nanotube clusters appear as chunks with extensions from $300 \mathrm{~nm}$ to several micrometres. The micrograph on the right shows also aqueous carbon nanotubes between a coated and an uncoated $\mathrm{Si}_{3} \mathrm{~N}_{4}$ membranes (chapter 3.1). It is taken with the STXM at $300 \mathrm{eV}$ making use of the high contrast just above the absorption edge of carbon. The recorded image size was $6 \times 10 \mu \mathrm{m}^{2}$ on $120 \mathrm{x} 200 \mathrm{pxl}^{2}$ with an exposure time of $10 \mathrm{~ms}$ per pixel.

A drop of carbon nanotubes originating from Rossendorf (rCNT) initially dispersed in water was imaged in dried state on a $\mathrm{Si}_{3} \mathrm{~N}_{4}$ membrane. Figure 6.6 presents three micrographs taken with the STXM at $280 \mathrm{eV}$ (figure 6.6 left), $286 \mathrm{eV}$ (middle), and $288.5 \mathrm{eV}$ (right). The exposure time was $24 \mathrm{~ms}$ per pixel, the image size $15 \mathrm{x} 15 \mu \mathrm{m}^{2}$ on $300 \times 300 \mathrm{pxl}^{2}$. The scale bar indicates $1 \mu \mathrm{m}$. Expectedly, the contrast in the image taken at $280 \mathrm{eV}$ and thus below the carbon absorption edge is extremely poor. The change in contrast between the micrograph taken at $285 \mathrm{eV}$ and $288.5 \mathrm{eV}$ gives information about a high content of $\mathrm{C}=\mathrm{C}$ bonds (Stöhr, 1996 (78), Ishii and Hitchcock, 1987 (31)). The 

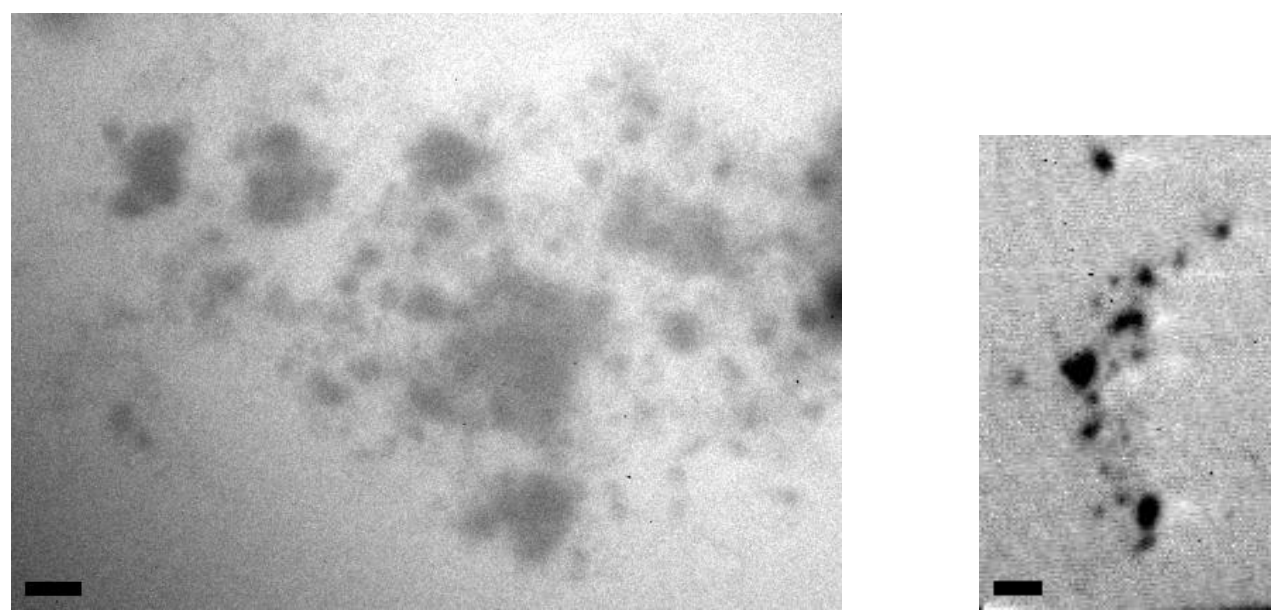

Fig. 6.5: Left: Micrograph of aqueous carbon nanotubes (baytubes) between two $\mathrm{Si}_{3} \mathrm{~N}_{4}$ membranes. The exposure time was $360 \mathrm{~s}$. Images taken with the CSXM at $E=368 \mathrm{eV}$. Right: Sample of aqueous carbon nanotubes as besides, but imaged at $300 \mathrm{eV}$ with the STXM. The recorded image size was $6 \times 10 \mu \mathrm{m}^{2}$ on $120 \times 200 \mathrm{pxl}^{2}$ with an exposure time of $10 \mathrm{~ms}$ per pixel. The scale bars indicate $1 \mu \mathrm{m}$.

resonance peak for the $\sigma^{*} \mathrm{C}=\mathrm{C}$ bond lies at $285.7 \mathrm{eV}$, where the rCNT sample shows maximum absorption. At $288.5 \mathrm{eV}$, the energy of the $\pi^{*} \mathrm{C}-\mathrm{C}$ bond, the absorption is weaker, but congruently distributed as in the micrograph taken at $286 \mathrm{eV}$ (additionally proven via a distribution map, but not shown). Thus, the distribution of the $\sigma^{*} \mathrm{C}=\mathrm{C}$ bonds and the $\pi^{*} \mathrm{C}$ - C bonds are proven to be uniform. Further spectroscopic analysis of carbon nanotubes (rCNT) can be found in Sedlmair et al., to be published (71).

It is noticeable that the assembly of the $\mathrm{rCNT}$ sample is different than the assembly of the baytubes sample shown in figure 6.5. While baytubes clusters in chunks, rCNT fold barely, but form a fine network. The network structure of the rCNT exists also in aqueous state as shown in Sedlmair et al., submitted (71). 

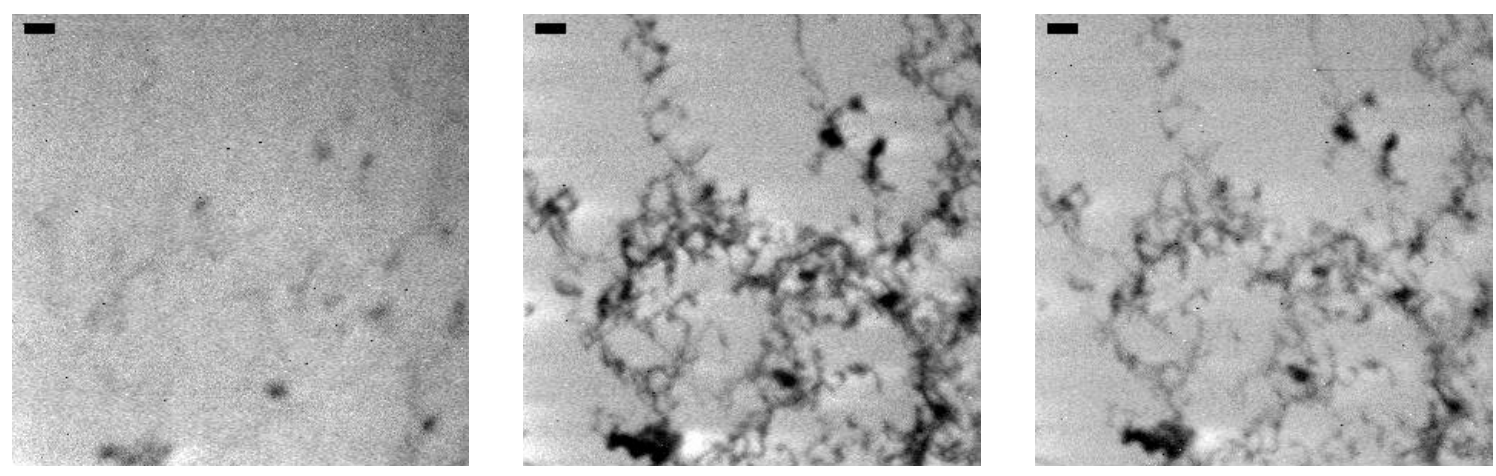

Fig. 6.6: Micrographs of carbon nanotubes (rCNT from Rossendorf, Germany) in dried state on a $\mathrm{Si}_{3} \mathrm{~N}_{4}$ membrane. Images taken at $280 \mathrm{eV}$ (left), $286 \mathrm{eV}$ (middle), and $288.5 \mathrm{eV}$ (right). The exposure time was $24 \mathrm{~ms}$ per pixel, the recorded image size $15 \times 15 \mu \mathrm{m}^{2}$ on $300 \times 300 \mathrm{px} \mathrm{l}^{2}$ $\left(298 \times 294 \mathrm{pxl}^{2}\right.$ presented). Images taken with the STXM. The scale bars indicate $1 \mu \mathrm{m}$.

\subsection{Kaolinite}

Kaolinite is one of the most common clay minerals and belongs to the 1:1 layer clays (chapter 5.1). Figure 6.7 shows two micrographs of a capillary tip with approximately $1.5 \mu \mathrm{m}$ diameter filled with a kaolinite dispersion taken with the XM-1 at $\mathrm{E}=707 \mathrm{eV}$ with a $14^{\circ}$ tilt angle between them (Gleber et al., 2009 (22)). Exposure times were $0.32 \mathrm{~s}$ with $1024 \mathrm{x} 1024 \mathrm{pxl}^{2}$. The tip was sealed by dipping it into a drop of silicone glue to prevent drying. Needle-like structures are visible, but without matching structures in both stereo images. This is due to the fact that only very small platelets could reach the tip of $1.5 \mu \mathrm{m}$ diameter. These small platelets show a good absorption contrast only when oriented parallel to the X-ray beam. The clay platelets oriented perpendicular to the light absorb the X-radiation so weakly that they are not visible. Thus, in this case it became impossible to identify the same edges in both images of the tilted pair in order to apply stereo analysis.

For imaging at the Fe L-3 edge, the capillary tip has to be very thin when investigating aqueous samples due to the higher X-ray absorption by the water. This leads to the problems discussed above for the sample shown in figure 6.7. Therefor, using a stack of $\mathrm{Si}_{3} \mathrm{~N}_{4}$ membranes is favourable in this case as visible in figure 6.8. Here, two different conglomerations of kaolinite clay platelets are depicted. The bigger kaolinite platelets laying parallel to the $\mathrm{Si}_{3} \mathrm{~N}_{4}$ membranes. As only very few haematite was added, the shape of the kaolinite platelets can be assumed as unmodified and thus representing the shape of pure kaolinite. When imaging at energies within the water window, the capillary tip can be larger. In the upper images of figure 6.9 , pure kaolinite platelets are 


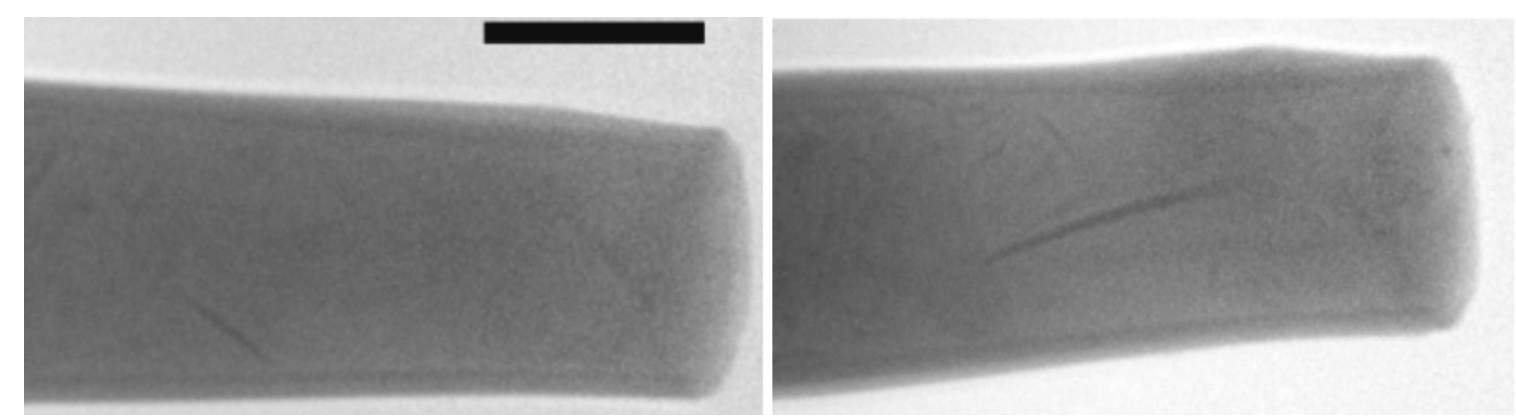

Fig. 6.7: Small particles of a kaolinite dispersion in a capillary tip with approximately $1.5 \mu \mathrm{m}$ diameter. The images were taken at $E=707 \mathrm{eV}$ at the XM-1. The tilt angle between the two images is $14^{\circ}$, the scale bar indicates $1 \mu \mathrm{m}$.

shown within a capillary tip with approximately $4.25 \mu \mathrm{m}$ diameter. These images were taken at $300 \mathrm{eV}$ with the STXM at BESSY. Both experiments are discussed in more detail in the following.

\subsubsection{Kaolinite with haematite}

Figure 6.8 shows image pairs of two clusters of kaolinite platelets within one sample of dispersed kaolinite mixed with haematite between two $\mathrm{Si}_{3} \mathrm{~N}_{4}$ membranes. The images on the left side of each pair were taken with the XM-1 at $704 \mathrm{eV}$ and the ones on the right side at $710 \mathrm{eV}$, exposure times were $9.5 \mathrm{~s}$ with $2048 \times 2048 \mathrm{pxl}^{2}$. When comparing both images of each pair, the ferrugineous haematite particles can be identified clearly in the respective right image, so it is not necessary to create elemental distribution maps dividing the images. It is noticeable that the haematite particles, which are easily mobile within the sample holder system due to their small size relatively to the water layer thickness, are located at the edges of the clay platelets. This is an indication for an electrostatic interaction of the positively charged haematite particles with the negatively charged edges of the clay platelets.

The chronological sequence of a haematite addition to a kaolinite dispersion is presented in figure 6.9, showing a conglomeration of kaolinite in a capillary tip of approximately $4.25 \mu \mathrm{m}$ diameter. The recorded image size was $20 \times 6 \mu \mathrm{m}^{2}$ at $400 \times 120 \mathrm{pxl}^{2}$ with $18 \mathrm{~ms}$ exposure time per pixel. The scale bar indicates $2 \mu \mathrm{m}$. The experiment was done with the STXM at $300 \mathrm{eV}$, permitting the use of a relatively thick capillary. The four presented micrographs are chronologically ordered from top to bottom and aligned to the position of the clay platelet situated in the middle if the imaged area. The first micrograph was taken shortly after injecting a kaolinite dispersion into the capillary. The 

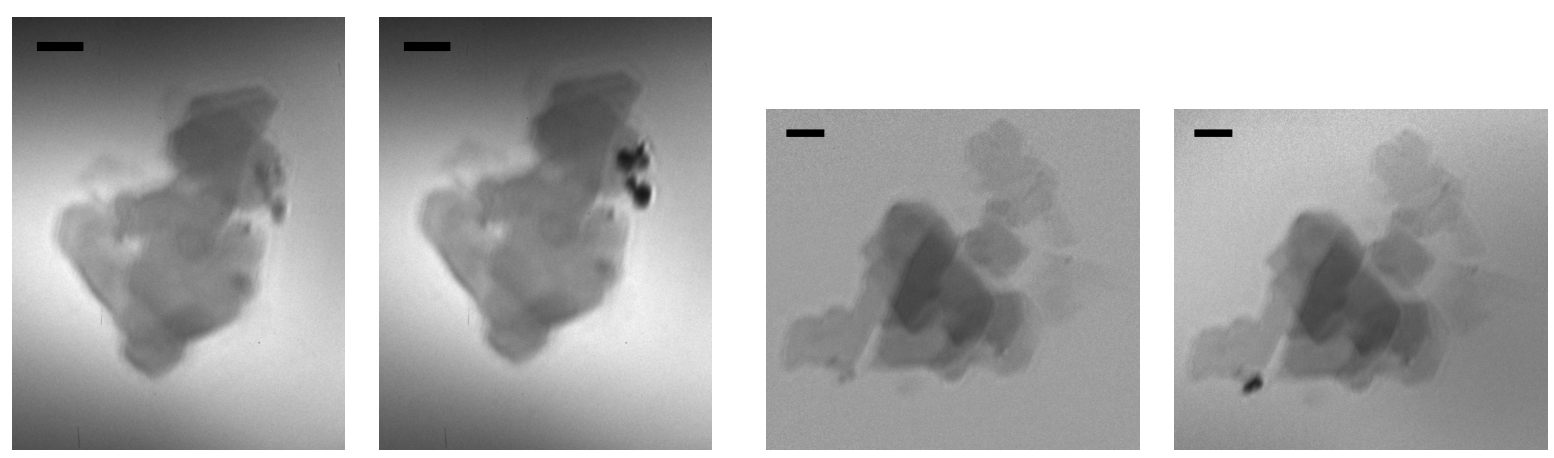

Fig. 6.8: Elemental mapping of haematite colloids within a dispersion of kaolinite platelets between two $\mathrm{Si}_{3} \mathrm{~N}_{4}$ membranes. Two pairs of images are presented, showing two different sample regions of the same sample. Images taken at $E=704 \mathrm{eV}$ (left micrograph of each pair) and $E=710 \mathrm{eV}$ (right micrograph of each pair). Haematite is clearly visible as dark structures in the right image of each image pair. The scale bars indicate $500 \mathrm{~nm}$. Images were taken with the XM-1.

second micrograph was taken from the same sample region one hour later. Morphological changes are clearly visible especially in the right side of the flock, where one kaolinite platelet was apparently still entering the capillary tip, causing a change in its position compared to the previous micrograph. Another half an hour later, thus $1.5 \mathrm{hrs}$ after the first micrograph, the third micrograph was taken. This accounts for the stability of the conglomeration adopted before the second micrograph. Subsequently, a haematite dispersion was injected secondary to the capillary. The fourth micrograph, which was taken $35 \mathrm{~min}$ after the addition of haematite, reveals a morphological change within the kaolinite flock. Haematite particles could not be detected, which can be explained by possibly lasting movement of the haematite particles and the poor iron contrast provided at $300 \mathrm{eV}$. The presented experiment included recording the respective stereo micrographs, which are not shown. Due to the high density of the conglomeration, a significant stereo analysis of this sample was not possible. Yet, the image series proves the possibility to document over several hours a time rate of change in the morphology of a sample in aqueous media with soft X-ray microscopy. Also the method for the inducement of morphological changes during a stereo soft X-ray microscopy experiment is proven. Note the slight clockwise turn of the outmost right particle in the capillary. 

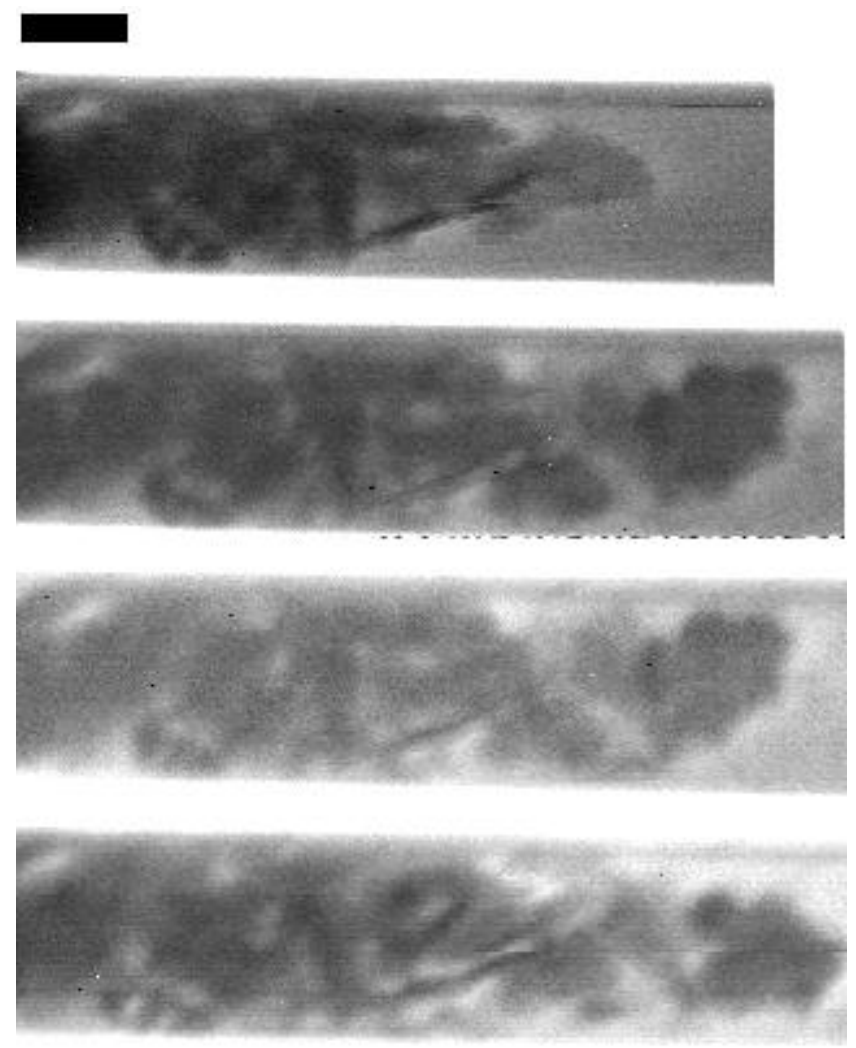

Fig. 6.9: Morphological changes within a conglomeration of kaolinite particles in a capillary tip with approximately $4.25 \mu \mathrm{m}$ diameter. First image (from top to bottom): Formation of kaolinite conglomeration imaged shortly after inserting dispersed kaolinite into the capillary. Second image: Same conglomeration as above, imaged one hour later. Morphological changes visible in comparison with the first image. Third image: Same conglomeration as above, imaged $1.5 \mathrm{hrs}$ after the first image. Configuration of the kaolinite flock was stable. Fourth image: Same conglomeration as above, but after addition of dispersed haematite. Fourth image taken $2.3 \mathrm{hrs}$ after the first image. Morphological changes again visible. All images were taken at $E=300 \mathrm{eV}$ at the STXM. The recorded image size was $20 \times 6 \mu \mathrm{m}^{2}$ at $400 \times 120 \mathrm{pxl}^{2}$ with $18 \mathrm{~ms}$ exposure time per pixel. The scale bar indicates $2 \mu \mathrm{m}$. 


\subsection{Nontronite}

Nontronite is a common 2:1 layer mineral with more than $30 \%$ of iron embedded (chapter 5.1). Two micrographs of nontronite dried onto a $\mathrm{Si}_{3} \mathrm{~N}_{4}$ membrane are presented in top of figure 6.10. The images were taken with the TwinMic with an exposure time of $40 \mathrm{~ms}$ per pixel and an image size of $100 \times 100 \mu \mathrm{m}^{2}$ on $250 \times 250 \mathrm{pixel}^{2}$. The scale bars indicate $6 \mu \mathrm{m}$. The left image is taken at $700 \mathrm{eV}$ and the right one at $708 \mathrm{eV}$, so the areas showing a strong X-ray absorption only in the right image can be identified as iron containing areas. Furthermore, some large platelets show an increase in contrast and are thereby identified as clay with iron incorporation. That is also true for the regions of smaller particles, covering densely distributed areas between larger platelets. The iron distribution is visualised in the corresponding element map shown in figure 6.10 below. The iron containing areas appear dark. It is noticable that unlike montmorillonite (chapter 6.5), nontronite is an iron rich clay as described in chapter 5.1. This is affirmed by the large areas of iron detected in figure 6.10 right and bottom.

Figure 6.11 shows also a nontronite sample, but imaged in an aqueous ambiance with the CSXM. The imaging energy was $368 \mathrm{eV}$ and the tilt angle between both micrographs is $14^{\circ}$ around the vertical image axis. The scale bar indicates $2 \mu \mathrm{m}$. Each image is a sum of 6 micrographs each taken with an exposure time of $60 \mathrm{sec}$. For each tilt angle, the series of single micrographs was considered with regard to sample movement. Thus, the stability of the sample was ascertained, which means that the changes of forms and distances detected when comparing the stereo micrographs base exclusively on the parallax of the spatially extended objects. In the right sample region, the shape of the clay is bulky, whereas in the left sample region a large, elongated structure is visible from which fine, linear structures stick out in a fan shape. This is due to the swelling properties of nontronite dispersed water.

Comparing figure 6.10 and figure 6.11, the morphological change of the nontronite sample is clearly visible due to the collapse of the fine structures of the swollen clay by drying.

\subsubsection{Nontronite with haematite}

Figure 6.12 shows a pair of stereo images of an aqueous sample of nontronite, where a haematite dispersion was added in a ratio of 1:10 before sandwiching it between two $\mathrm{Si}_{3} \mathrm{~N}_{4}$ membranes. The micrographs were taken with the CSXM at $368 \mathrm{eV}$ with a tilt angle of $14^{\circ}$. For the first position (figure 6.12 left), 13 micrographs of 60 seconds 

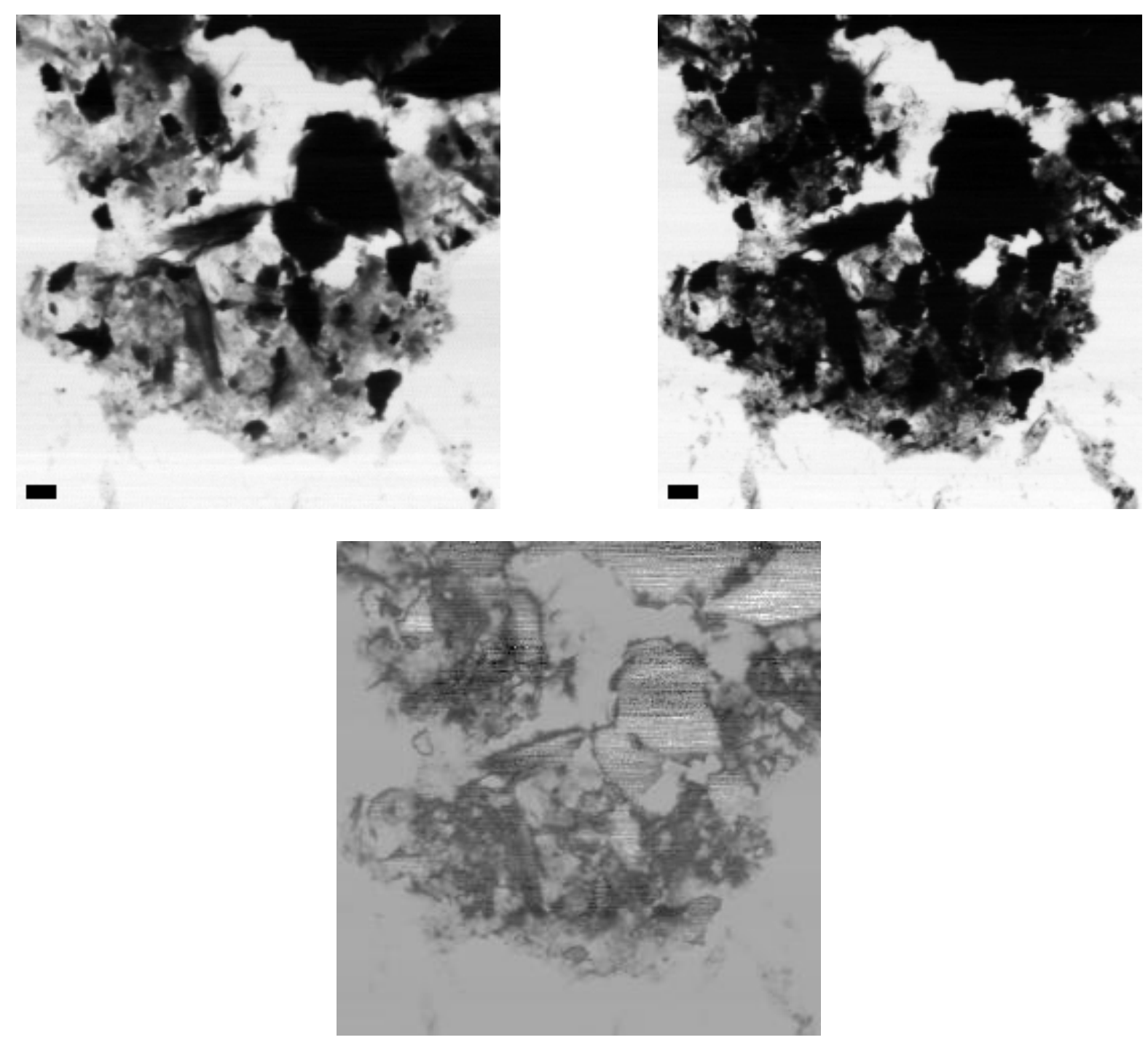

Fig. 6.10: Micrographs of nontronite around the $\mathrm{Fe} L$ absorption edge and resulting iron distribution map. Top: Micrographs of nontronite dried on a $\mathrm{Si}_{3} \mathrm{~N}_{4}$ membrane. Images taken at $700 \mathrm{eV}$ (left) and $708 \mathrm{eV}$ (right). The exposure time is $40 \mathrm{~ms}$ per pixel, the recorded image size $100 \times 100 \mu^{2}$ on $250 \times 250$ pixels $^{2}$ ( $240 \times 245$ pixels $^{2}$ shown). Images taken with the TwinMic. The scale bars indicate $6 \mu \mathrm{m}$. Bottom: Corresponding iron distribution map depicting iron containing areas dark. Regions of complete absorption in both images due to sample thickness appear grey with dark and light stripes (visible e.g. in the top right corner). 


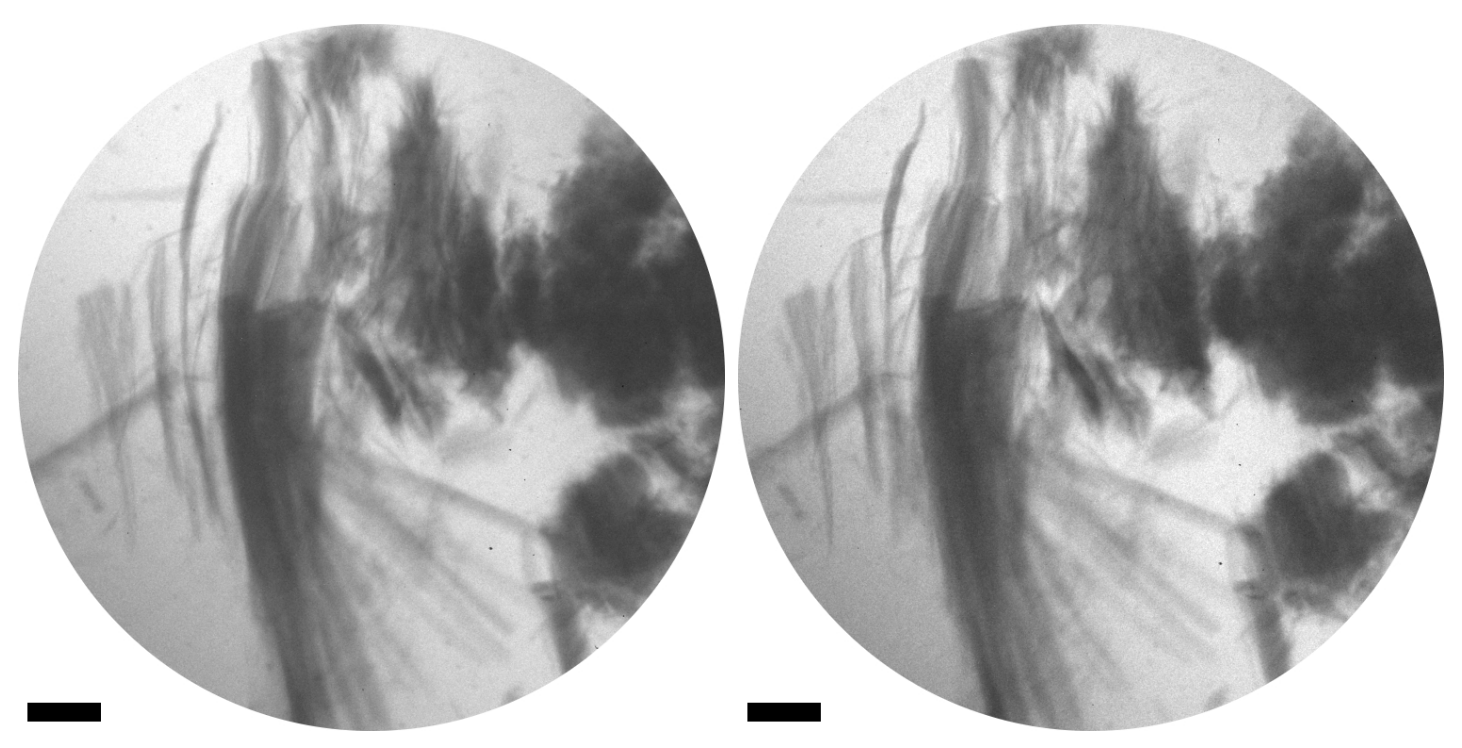

Fig. 6.11: Pair of stereo micrographs of an aqueous nontronite particles between two $\mathrm{Si}_{3} \mathrm{~N}_{4}$ membranes. The tilt angle is $\Delta \theta=14^{\circ}$ around the vertical image axis (suitable for stereo vision). Images taken with the CSXM at $E=368 \mathrm{eV}$, diameter of image field approximately $20 \mu \mathrm{m}$. Each image is a sum of 6 micrographs each with $60 \mathrm{~s}$ exposure time. The scale bars indicate $2 \mu \mathrm{m}$.
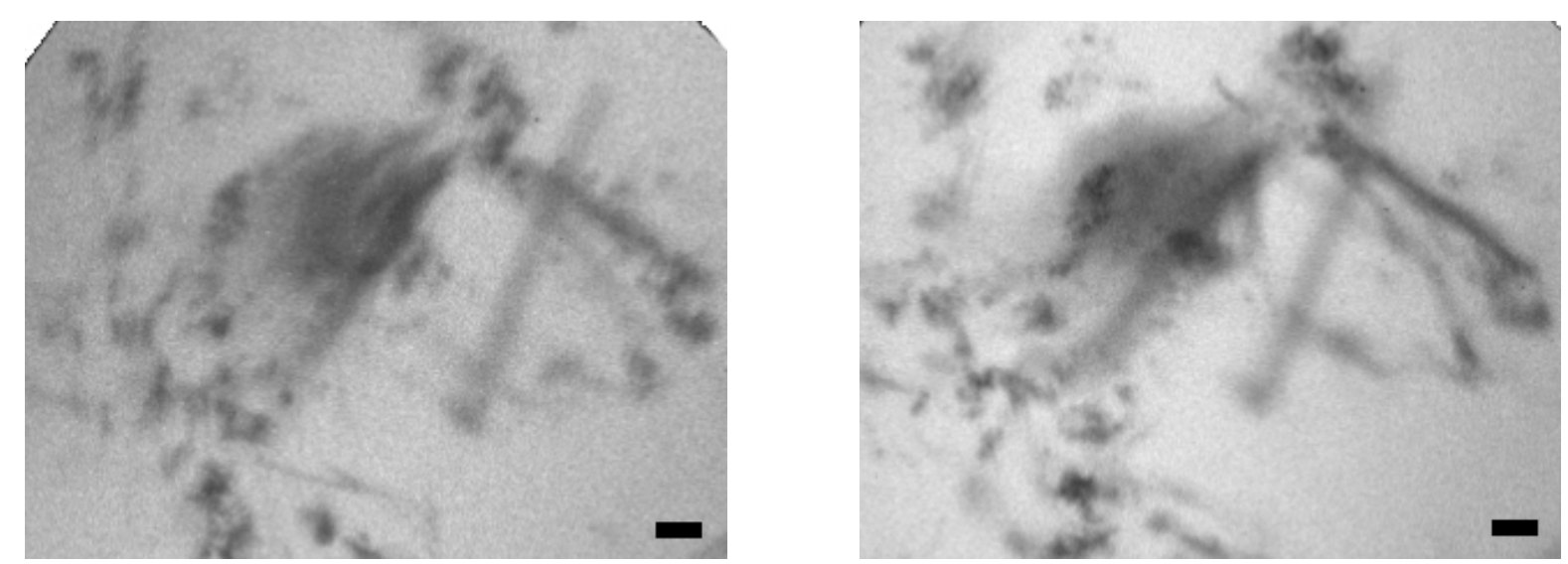

Fig. 6.12: Pair of stereo images of an aqueous sample of nontronite mixed with haematite between two $\mathrm{Si}_{3} \mathrm{~N}_{4}$ membranes. Small clusters of haematite particles are visible as black dots. The tilt angle is $\Delta \theta=14^{\circ}$ around the vertical image axis (suitable for stereo vision). Images taken with the CSXM at $E=368 \mathrm{eV}$. Each image is a sum of 6 micrographs with $60 \mathrm{~s}$ exposure time. The scale bars indicate $1 \mu \mathrm{m}$. 

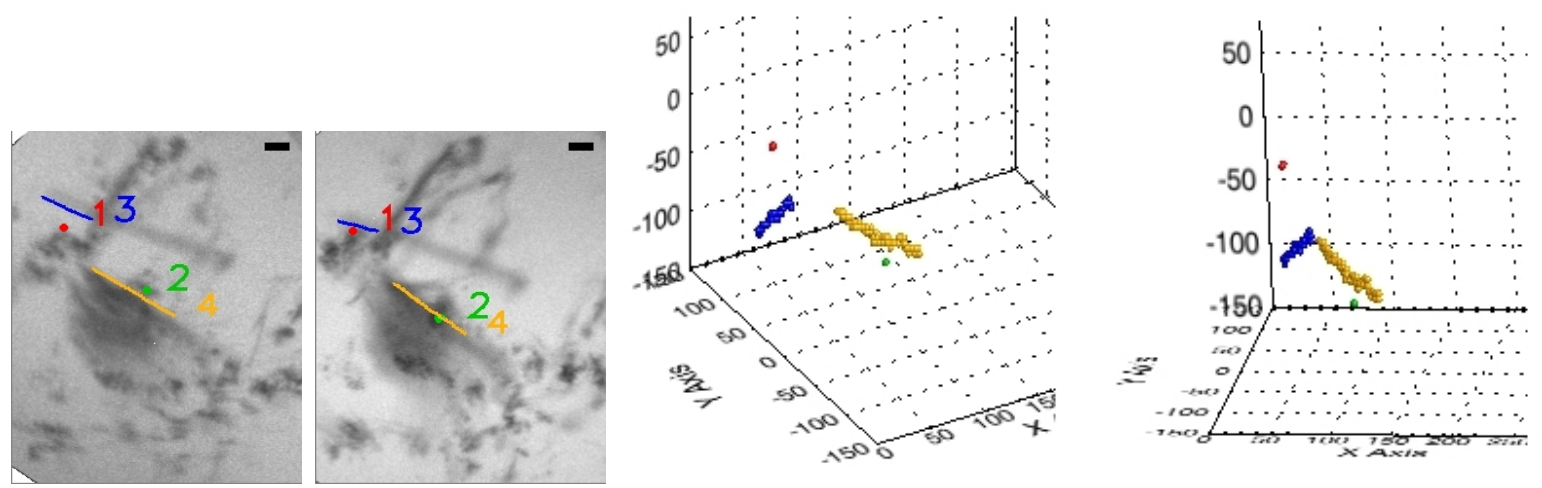

Fig. 6.13: xstereo analysis of an aqueous nontronite sample with haematite. Left side: Processing of the stereo images of the aqueous sample of nontronite mixed with haematite shown in figure 6.12 with xstereo. Two haematite structures are marked as dots (1 (red) and 2(green)), and two edges of nontronite platelets are marked as lines (3 (blue) and 4(yellow)) for analysis. Here, the tilt axis corresponds to the horizontal image axis. Right side: Two projections of the stereo plot obtained by xstereo, depicting the spatial distribution of the marked structures. (Details in chapter 6.4.1.)

exposure time each were recorded. As the first 5 micrographs revealed movements of the particles, just the last 8 micrographs were added to form the presented high contrast image, resulting in a total exposure time of 480 seconds. In the micrographs, extended nontronite platelets similar to those of figure 6.11 can be identified. Small clusters of haematite particles are visible as black spheres. The other smaller clusters are either nontronite particles or clusters of haematite, the classification is not possible without elemental mapping.

For stereo analysis, two haematite structures are marked as dots (1 (red) and 2 (green)), and two edges of nontronite platelets are marked as lines (3 (blue) and 4 (yellow)) for analysis with xstereoas shown in figure 6.13. Here, the tilt axis corresponds to the horizontal image axis. On the right side of figure 6.13, two projections of the stereo plot obtained by xstereo, representing the spatial distribution of the marked structures. The calculated distances between the point-shaped haematite particle 1 (red) and nontronite platelet edge 3 (blue) is $2.83 \mu \mathrm{m}$. It is noticeable that in the right projection image (figure 6.12 right), this haematite particle seems to be attached to the nontronite platelet.

In case of the cluster marked as structure 2 (green), the identification as haematite particle is less reliable than for structure 1 (red). The point-shaped aspect of this cluster in the left micrograph (figure 6.12) supports the assumption of a cluster of haematite 

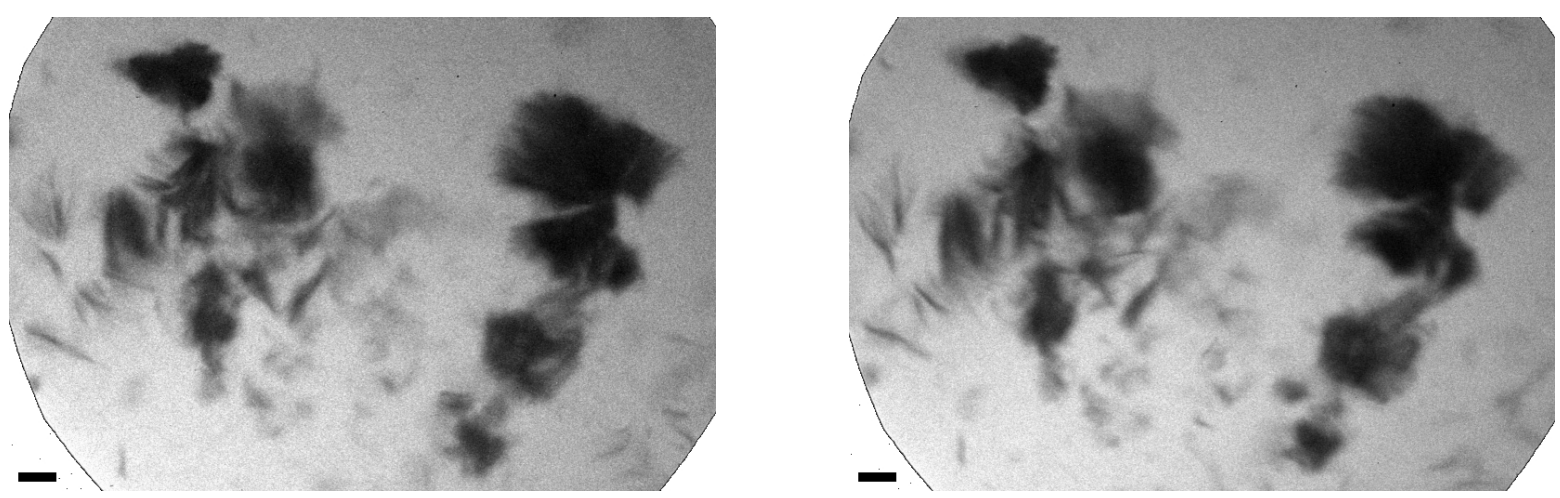

Fig. 6.14: Stereo micrographs of an aqueous sample of montmorillonite particles between two $\mathrm{Si}_{3} N_{4}$ membranes. The tilt angle is $\Delta \theta=14^{\circ}$ around the horizontal image axis. The exposure time was $360 \mathrm{~s}$ per micrograph, taken with the CSXM at E=368eV. The scale bars indicate $1 \mu \mathrm{m}$.

particles. The distance between the cluster 2 (green) and nontronite platelet edge 4 (yellow) was revealed to be $640 \mathrm{~nm}$.

\subsection{Montmorillonite}

Montmorillonite is the most common member of the group of 2:1 clay minerals (chapter 5.1). Figure 6.14 shows a pair of stereo micrographs of aqueous montmorillonite between two $\mathrm{Si}_{3} \mathrm{~N}_{4}$ membranes. The micrographs are taken with the CSXM at $368 \mathrm{eV}$ with an exposure time of $360 \mathrm{~s}$. The left micrograph was taken before, the right micrograph after tilting the object by $14^{\circ}$. The tilt axis equates to the horizontal image axis. The scale bars indicate $1 \mu \mathrm{m}$. Both large platelets are visible and also many fine structures which are attached to conglomerations as well as spread over the presented sample area. The assembly of clay platelets of more than $1 \mu \mathrm{m}$ extensions on the right side of the image shows visible parallax.

Figure 6.15 (left and middle) shows a pair of stereo micrographs of another sample of aqueous montmorillonite between two $\mathrm{Si}_{3} \mathrm{~N}_{4}$ membranes. The micrographs were taken with the CSXM at $368 \mathrm{eV}$ with an exposure time of $300 \mathrm{~s}$. The left micrograph was taken before, the right micrograph after tilting the object by $14^{\circ}$. The tilt axis equates to the vertical image axis. The scale bars indicate $2 \mu \mathrm{m}$. In comparison to the stereo image presented in figure 6.14, smaller structures appear sharply in considerable number. Furthermore, the parallax in the stereo pair shown in figure 6.15 is barely noticeable. This is due to a very thin sample, where the particles lie level with each other. Small 

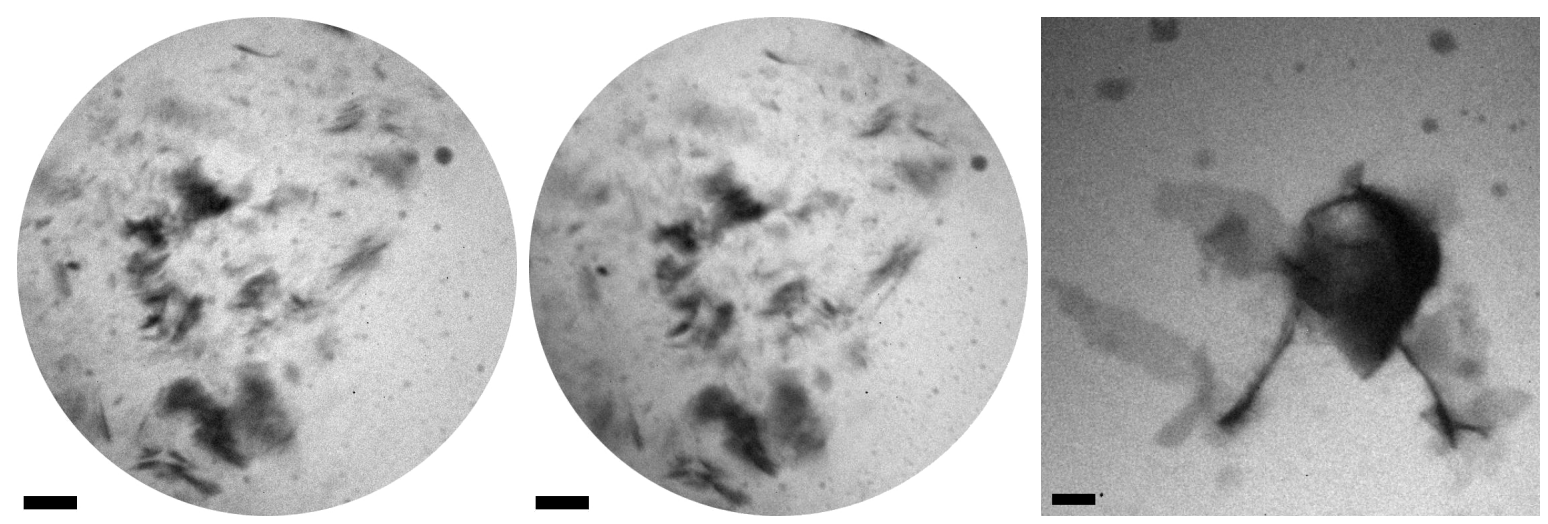

Fig. 6.15: Montmorillonite. Left and middle: Stereo micrographs of an aqueous sample of montmorillonite particles shortly before drying. The tilt angle is $\Delta \theta=14^{\circ}$ around the vertical image axis. The images were taken with an exposure time of $300 \mathrm{~s}$ each with the CSXM at $E=368 \mathrm{eV}$. The scale bars indicate $2 \mu \mathrm{m}$. Right: Same sample as besides, showing another sample region after drying. The exposure time was $180 \mathrm{~s}$, the scale bar indicates $1 \mu \mathrm{m}$.

particles are clamped between the $\mathrm{Si}_{3} \mathrm{~N}_{4}$ membranes and thus steady enough to be imaged.

A further change in structure is observed in the dried state of the montmorillonite sample as revealed in the right image shown in figure 6.15. Another region of the same sample as shown besides is presented, imaged after complete drying. The exposure time was $180 \mathrm{~s}$, thus less than before as no water layer had to be transmitted. All particles are in focus. Very fine structures are visible, due to the complete immobility of the structures deflated in the dried state. The scale bar indicates $1 \mu \mathrm{m}$.

Comparing the images of aqueous samples of pure montmorillonite (figures 6.14 and 6.15 left) to those of nontronite (figure 6.11), it becomes apparent that nontronite consists of large structures, while montmorillonite also contains small particles. Thus, unlike shown for a mixture of nontronite and haematite in figure 6.12, haematite particles within a montmorillonite sample cannot be identified just by shape, as discussed in the following chapter.

\subsubsection{Montmorillonite with haematite}

An aqueous montmorillonite dispersion mixed with haematite is presented in figure 6.16. Each micrograph was taken with an exposure time of $600 \mathrm{~s}$ at $368 \mathrm{eV}$ with the CSXM. The left and the right micrograph form a stereo pair with a tilt angle of $14^{\circ}$ around the 

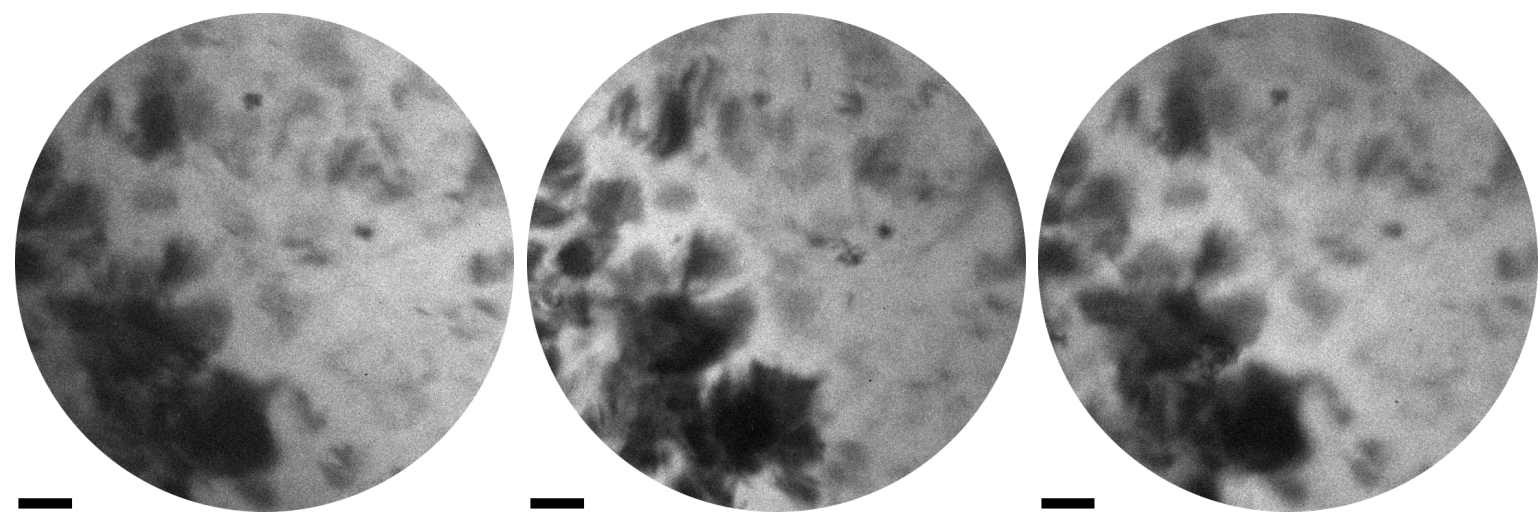

Fig. 6.16: Aqueous sample of montmorillonite particles with haematite colloids between two $\mathrm{Si}_{3} \mathrm{~N}_{4}$ membranes. Left and middle: Pair of stereo micrographs with a tilt angle of $\Delta \theta=14^{\circ}$ around the vertical image axis. Right: Micrograph as the left one but taken after the tilted micrograph (middle). Images taken with the CSXM at $\mathrm{E}=368 \mathrm{eV}$ and $600 \mathrm{~s}$ exposure time. The scale bars indicate $2 \mu \mathrm{m}$.

vertical image axis. Changes in the projection images due to the tilt are visible on the left and lower region of the sample. As these micrographs were taken with continuous exposure times of $600 \mathrm{~s}$ instead of exposure sequences, a third micrograph was recorded to exclude sample movement during the exposure of the stereo image pair (figure 6.16 right).

In these micrographs, no haematite particles can be identified because the samples of pure montmorillonite possess small structures of similar shape as visible in figure 6.16. Only elemental mapping reveals a reliable discrimination between haematite and clay particles as shown in the following.

In the top images of figure 6.17, a montmorillonite sample mixed with haematite between two $\mathrm{Si}_{3} \mathrm{~N}_{4}$ membranes and imaged at $700 \mathrm{eV}$ and $707 \mathrm{eV}$ can be seen (Gleber et al., 2009 (22)). Exposure times were $0.45 \mathrm{~s}$ with an image size of $1024 \mathrm{x} 1024 \mathrm{pix}^{2}$. Although due to a slight mismatch in energy the contrast is not optimum, the haematite particles can still be seen in the right image when comparing it with the left image. The discrimination between soil and haematite particles is supported and complemented by an elemental distribution map presented in the bottom image of figure 6.17. Here, haematite particles appear clearly as dark dots and are revealed also in positions not evident in the absorption images. This sample was then tilted by $14^{\circ}$ around a horizontal axis and imaged again at both energies. Figure 6.18 shows a detail of the sample shown in figure 6.17, one selected from the right image of figure 6.17 , the other one selected from an image of the same region taken at $707 \mathrm{eV}$ after tilting the sample. For stereo analysis, 

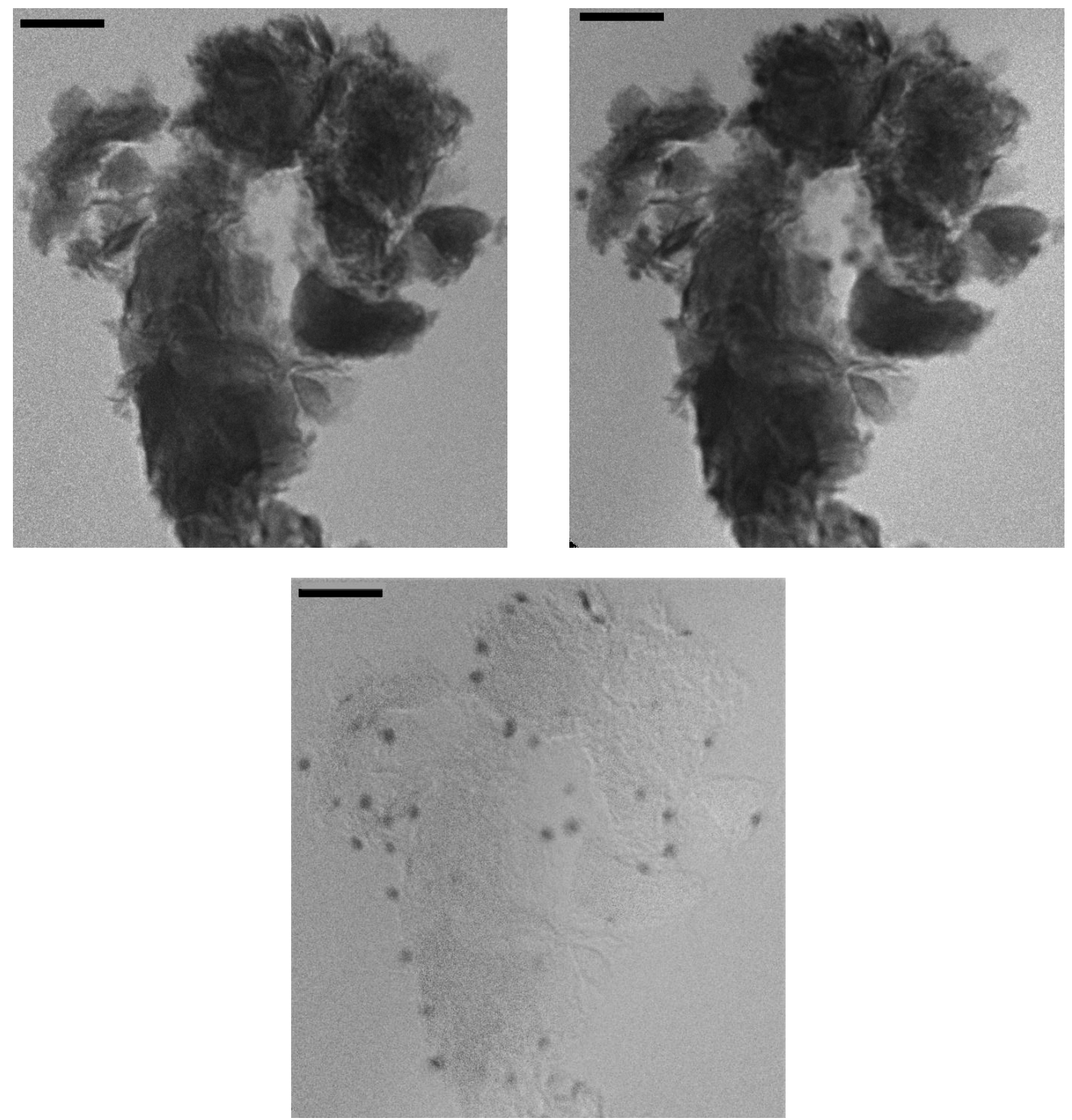

Fig. 6.17: Aqueous sample of montmorillonite particles with haematite colloids between two $\mathrm{Si}_{3} \mathrm{~N}_{4}$ membranes. Top: Micrographs were taken at $E=700 \mathrm{eV}$ (left) and $E=707 \mathrm{eV}$ (right) with an exposure time of $0.45 \mathrm{~s}$. Bottom: Elemental distribution map where haematite particles appear as dark dots. The scale bars indicate $1 \mu \mathrm{m}$. 

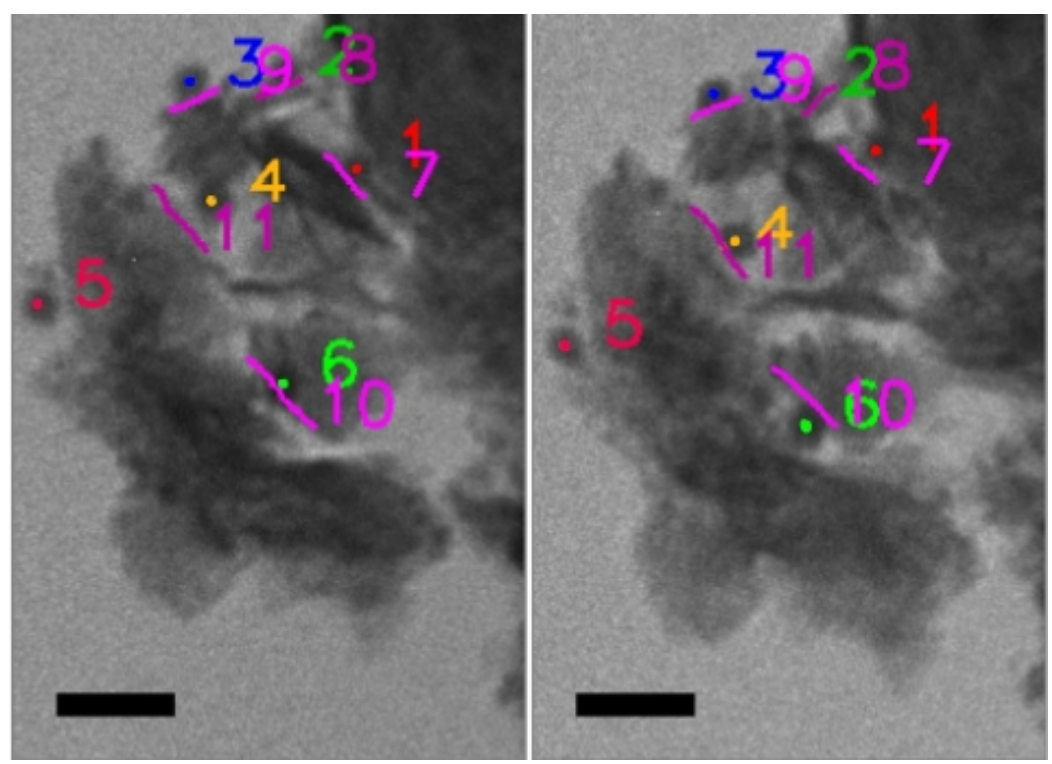

Fig. 6.18: Detail of the montmorillonite sample shown in figure 6.17 viewed with an angular difference between the images of $14^{\circ}$ around a horizontal tilt axis, taken at $E=707 \mathrm{eV}$. Lines, points, and edges are marked for analysis (details in section 6.5). The scale bar indicates $500 \mathrm{~nm}$.
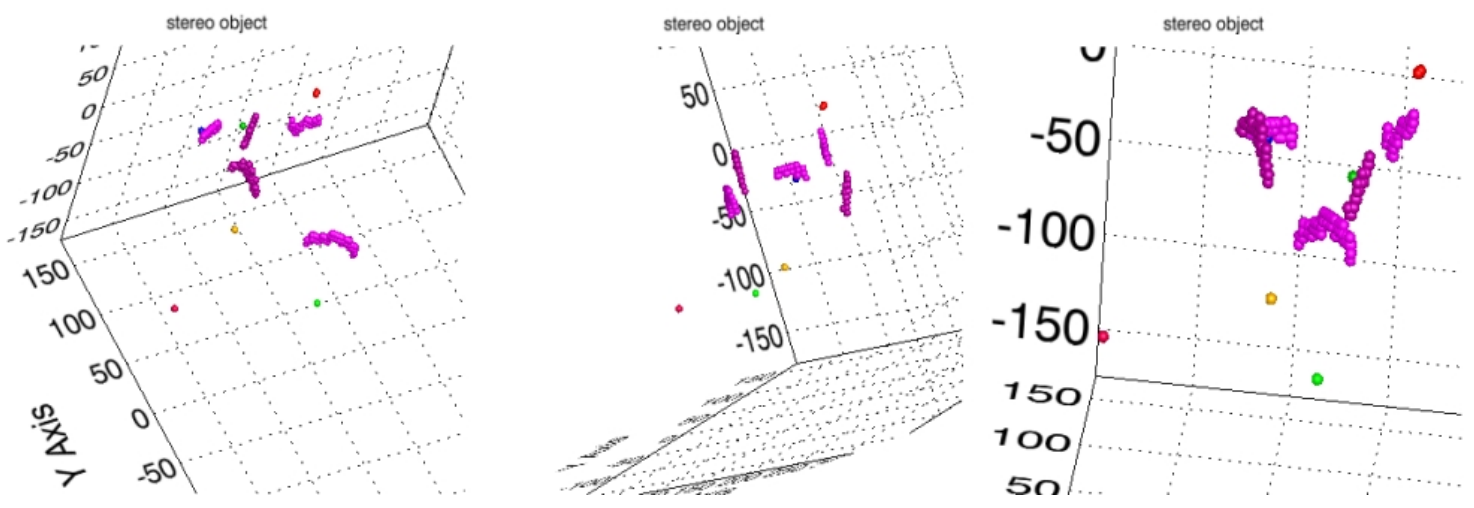

Fig. 6.19: Plot showing the points and edges marked in figure 6.18 presented under three different viewing angles. The unit of the axis is pixels. In the processed images, 10 pixels relate to $90 \mathrm{~nm}$. 
haematite particles were identified and marked as single dots in different colours, whereas nearby edges of clay platelets were marked as lines in magenta (figure 6.18), and then processed with xstereo. The created plot of the three-dimensional arrangements of the marked structures is presented in figure 6.19. Three different viewing angles have been chosen to depict proximity relations. The units of this plot are pixels, with one pixel corresponding to $9 \mathrm{~nm}$. Distances between the structures were determined. So, it became clear that the haematite particles, whose centres are marked as structures number 2 (dark green) and 3 (blue), are particles attached to the edges of clay platelets, which are marked partially as number 8 (dark magenta) and 9 (light magenta), respectively. The distances have been determined to $60 \mathrm{~nm}$ between haematite particle number 2 and clay platelet edge number 8 , and $80 \mathrm{~nm}$ between haematite particle number 9 and clay platelet edge number 9 . Taking the radii of the haematite particles into account, this corresponds to a direct attachment. Haematite particle number 1 (red) is not as close to the clay edge number 7 (light magenta) as it seems in the left image of figure 6.18 , but has a distance of $180 \mathrm{~nm}$. The minimum distance between the centre of the haematite particle number 4 (orange) and the platelet edge number 11 (dark magenta) is $630 \mathrm{~nm}$. This cannot be seen by simply looking at one transmission image, rather, these structures seem to be attached to each other. This holds true also for particle number 6 (light green) and edge number 10 (light magenta), where the real distance is $600 \mathrm{~nm}$. This spatial arrangement is visualised in figure 6.19, where particles and edges are plotted in a three-dimensional coordinate system. Here, the haematite particles number 4, 5, and 6 seem to be in one plane perpendicular to the z-direction, thus possibly attached to one of the support membranes. The haematite particles revealed in the elemental mapping at overlapping positions with the soil particles are probably either attached to the basal planes of the soil platelets, as the clay shows a permanent negative charge there, or they are attached to the edges of overlapping clay platelets. In any case, the conformation of these particles proof that the system of the clay particles between two $\mathrm{Si}_{3} \mathrm{~N}_{4}$ membranes allows for various positions of the smaller particles, e.g. haematite, and is therefore representative for a natural system. Conclusions on the revealed adsorption of the heamatite particles on the edges of the clay platelets with regards to the $\mathrm{pH}$ value of the sample are presented in chapter 6.9.

For the investigation of induced dynamical behaviour, a capillary was filled with a montmorillonite dispersion. Figure 6.20 presents two micrographs. The left image shows a pure dispersion of montmorillonite, taken with the STXM at $400 \mathrm{eV}$. The exposure time was $40 \mathrm{~ms}$ per pixel, and the recorded image size $20 \times 12 \mu \mathrm{m}^{2}$ at $400 \times 230 \mathrm{pix}^{2}$, 

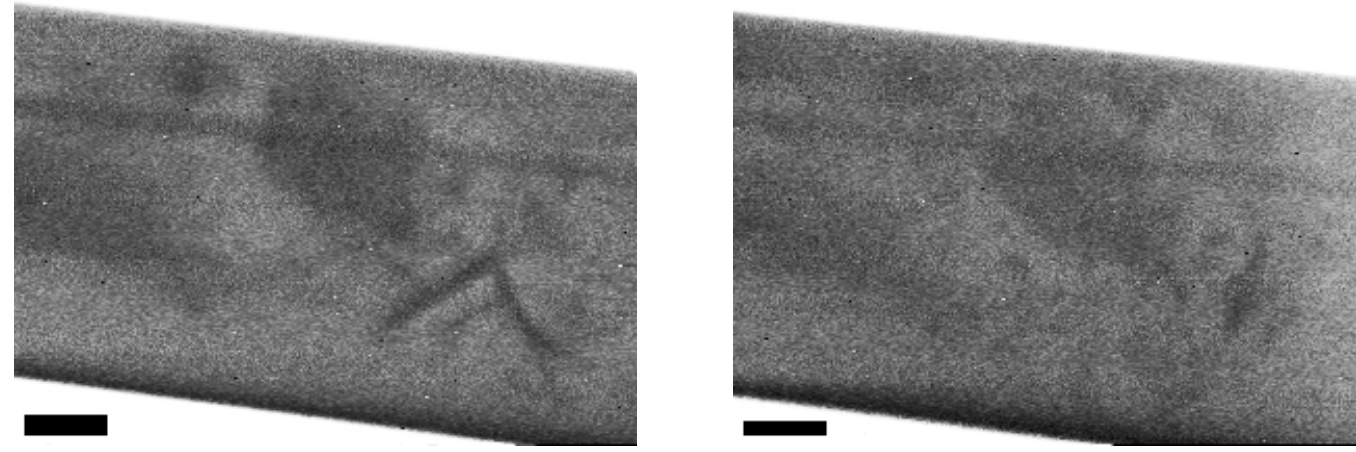

Fig. 6.20: Addition of haematite to an aqueous sample of montmorillonite in a capillary tip, revealing structural changes after the addition of haematite. Left: Pure dispersion of montmorillonite. Right: Same sample position as besides, but imaged $1.5 \mathrm{hrs}$ after the addition of a haematite dispersion, causing conformational changes. Images taken at $E=400 \mathrm{eV}$ with the STXM. The exposure time was $40 \mathrm{~ms}$ per pixel and the recorded image size $20 \times 12 \mu \mathrm{m}^{2}$ at $400 \times 240 \mathrm{pix}^{2}\left(300 \times 240 \mathrm{pix}^{2}\right.$ shown). The scale bars indicate $2 \mu \mathrm{m}$.

of which $300 \times 230 \mathrm{pix}^{2}$ are shown. The scale bar indicates $2 \mu \mathrm{m}$. Due to the X-ray absorption of the $10 \mu \mathrm{m}$ thick water layer in the capillary, the image quality is rather low. In the capillary of pure montmorillonite, two large particles and a smaller one are visible in the left and in the middle of the image, and on the right side, an assembly of particles, probably clay platelets with their basal planes parallel to the X-rays. The glass filament of the capillary is also visible. While still mounted to the X-ray microscope, dispersed haematite was injected additionally to the capillary. Subsequently, $1.5 \mathrm{hrs}$ after the addition of haematite to the capillary, another micrograph was taken of the same sample region as before. The sample conformation of the larger particles has drifted very little, while the assembly of particles on the right side is totally different. As previously shown in figure 6.9 for a kaolinite sample, this demonstrates the ability to image conformational changes within dispersed montmorillonite induced during an X-ray imaging experiment with the STXM. The capillary again proves to be a suitable sample holder, where conglomerations of particles stay sufficiently stable to be imaged after the injection of additional samples, but with enough space for subsequently injected very small particles to reach the tip and for parts of the clusters to react with conformational changes. To clearly identify the changes due to haematite particles, elemental mapping has to be applied. The realisation is discussed in chapter 7 . The suggestive combination with stereo imaging is shown in following examples. 

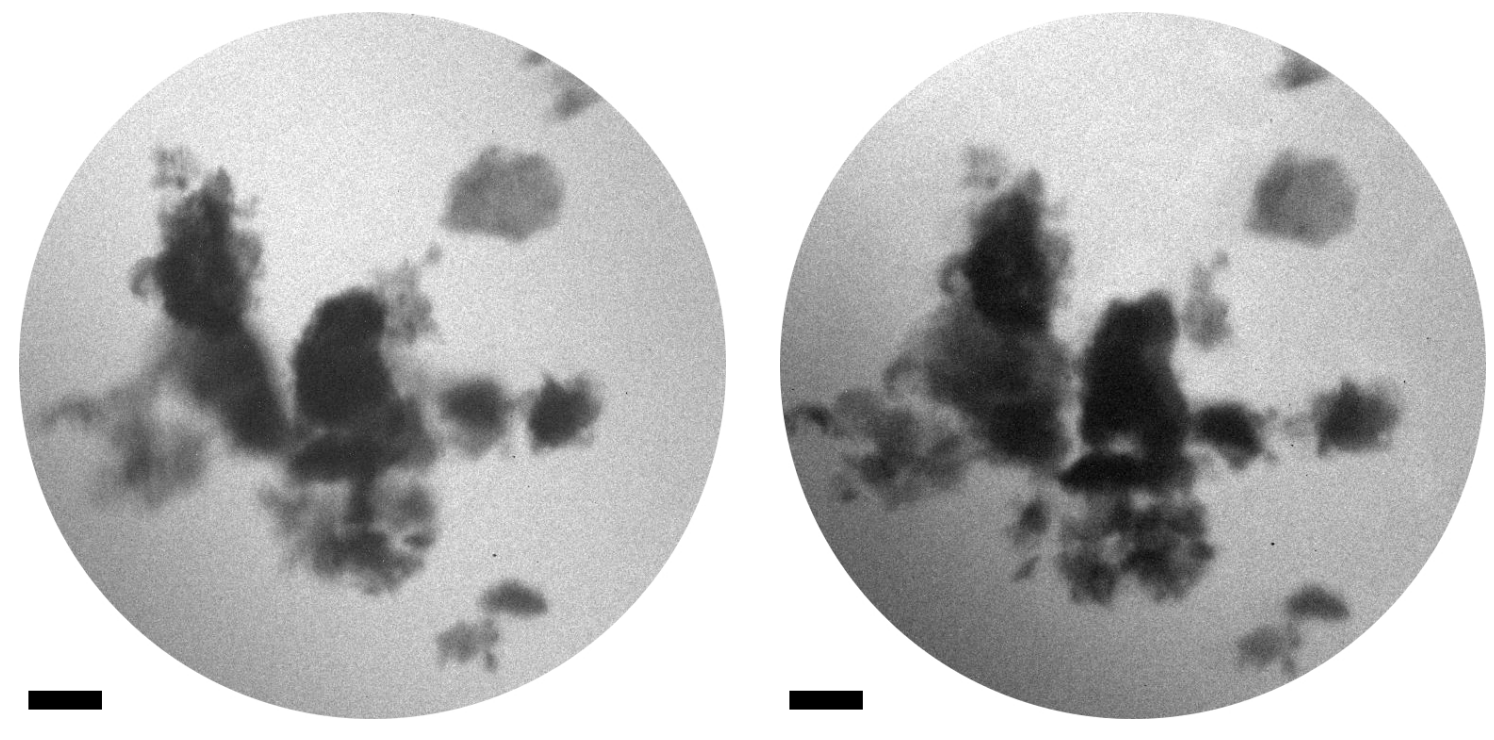

Fig. 6.21: Aqueous sample of montmorillonite particles with carbon nanotubes (baytubes) between two $\mathrm{Si}_{3} N_{4}$ membranes. The tilt angle is $\Delta \theta=14^{\circ}$ around the vertical image axis. Each image is a sum of 6 micrographs each with $60 \mathrm{~s}$ exposure time. Images taken with the $C S X M$ at $E=368 \mathrm{eV}$. The scale bars indicate $2 \mu \mathrm{m}$.

\subsubsection{Montmorillonite with carbon nanotubes}

Also carbon nanotubes were added to montmorillonite samples. Figure 6.21 presents a pair of stereo micrographs of an aqueous montmorillonite sample mixed with dispersed carbon nanotubes (baytubes) between two $\mathrm{Si}_{3} \mathrm{~N}_{4}$ membranes. The images were taken with the CSXM at $368 \mathrm{eV}$. The tilt angle is $14^{\circ}$ around the vertical image axis. The scale bar indicates $2 \mu \mathrm{m}$. The spatial extension of the sample becomes apparent both in the parallax and in the focused and unfocused structures. The large platelets situated top right and in the middle of the image are approximately focused in both stereo micrographs, as well as the smaller structure situated down right of the image area. However, the large platelet shown top left is focused only in the left micrograph, whereas the cluster region beneath it appears focused only in the right micrograph. Also the sample structures beneath the platelet situated in the middle of the image area are focused only in the right side micrograph. However, possible particle motion has to be taken into account as well. The particular sample regions are discussed in detail in the following.

Again, each image of figure 6.21 is formed by the addition of 6 micrographs taken subsequently with $60 \mathrm{~s}$ exposure time each, to exclude or rather detect sample movements during the complete stereo experiment, as the CSXM requires relatively long exposure 
times of several minutes for high resolution images of aqueous samples (chapter 4.1.2). The first and the last micrograph of each image series is presented in figure 6.22 top and middle. The images shown on left side correspond to the left micrograph in figure 6.21, the right images accordingly. The left image series was taken within $12 \mathrm{~min}$ before tilting the sample holder. $12 \mathrm{~min}$ afterwards, the second series of stereo images (figure 6.22 top and middle right) was recorded within $13 \mathrm{~min}$, resulting in a total time span of 31 min. Both top images show the first micrographs and the middle images the sixth micrographs of the respective image series. For a final check for possible sample movements, a further micrograph was recorded subsequent to both series of stereo images at the initial tilt position of the sample and is shown in figure 6.22 bottom left. The time lag between recording this micrograph and the first one of the stereo experiment (figure 6.22 top left) is $49 \mathrm{~min}$. The CSXM stereo stage does not provide a sub micrometre range accuracy (chapter 4.1.2), so it is necessary to align the image field after tilting. Therefore, the control micrograph had to be aligned, which was done according to the corresponding micrographs (figure 6.22 top and middle left) on the basis of the large particle in the centre of the sample area. This alignment is in good agreement with most of the apparently motionless particles. The focal plane of this micrograph is apparently slightly different than in the micrographs taken before as stereo tilt series under this tilt angle. The image in figure 6.22 bottom right defines regions of interest for evaluating the stability of the sample during stereo imaging in detail.

In the region marked as number 1, a cluster of small particles is visible. As already mentioned above, the cluster appears defocused in the left image series, and focused in the right image series. The arrangement seems to be stable as a whole, except slight morphological changes in the left half visible in the first image series (figure 6.22 top and middle left), and minor shrinkages on two edges of the structures in the lower part visible in the second image series (figure 6.22 top and middle right). The constant distance to the strongly absorbing large particle above this assembly confirms this impression. However, the whole assembly drifts within the first image series by approximately $250 \mathrm{~nm}$ down, while the drift of the right side is about $80 \mathrm{~nm}$ less. The structures on the left side drift additionally about $130 \mathrm{~nm}$ to the left. (These lengths were determined by measuring the positions of different particular structures, recognisable in all images, with an estimated accuracy of $4 \mathrm{pxl}$, according to $51 \mathrm{~nm}$.) Both drifts are not detected within the second image series. The y-values of the structures agree with the corresponding y-values measured in the last micrograph of the first image series. This holds also true for the $y$-values measured in the control image (figure 6.22 bottom left), except for 

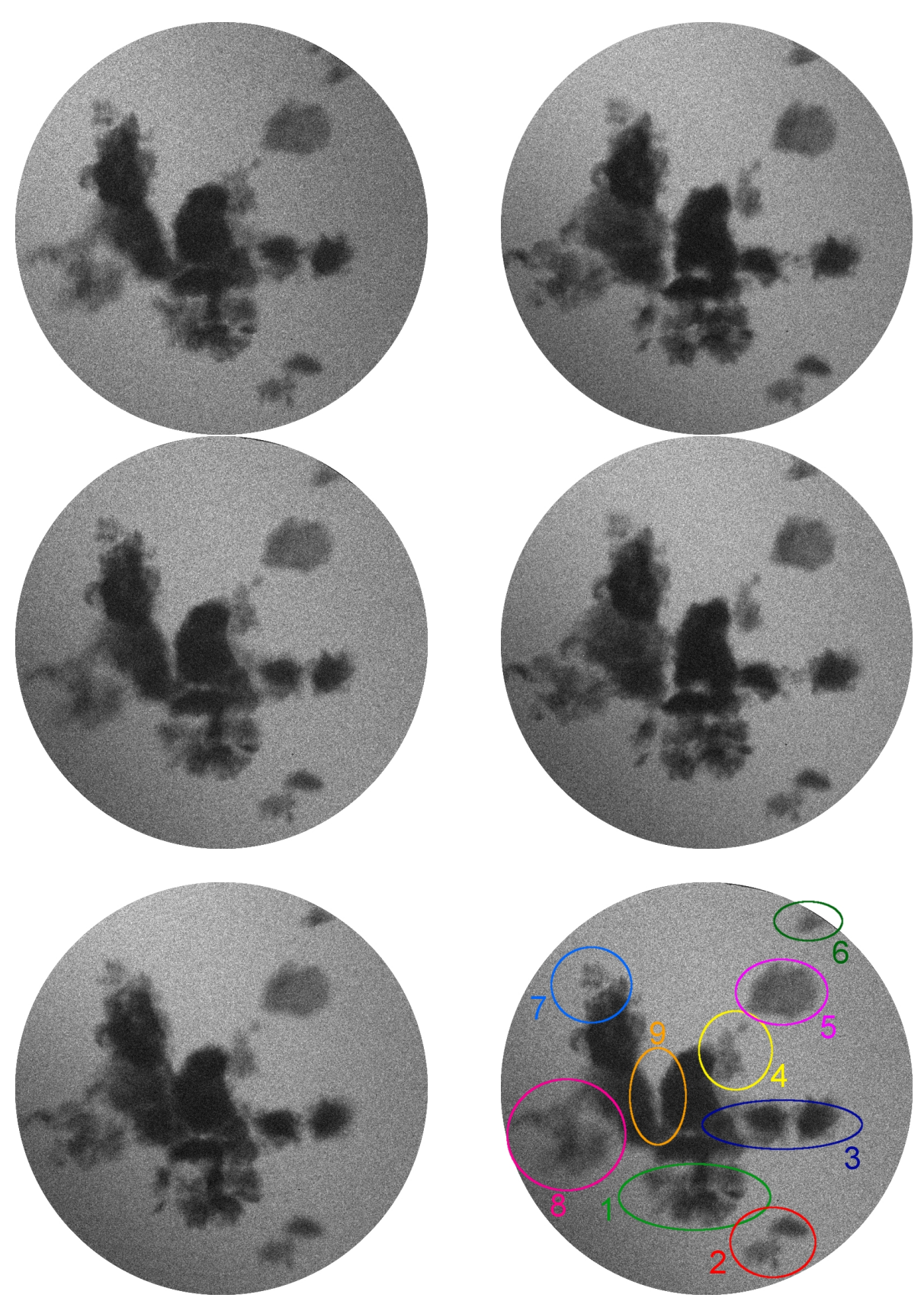

Fig. 6.22: Identification of sample movement during the stereo experiment for the images shown in figure 6.21. Top and middle: Sequences of the added stereo images of the aqueous sample of montmorillonite with carbon nanotubes (baytubes) shown in figure 6.21 with $60 \mathrm{~s}$ exposure time. Images on left side correspond to left image in figure 6.21, right images accordingly. Left image series taken before tilting the sample for recording series of images shown right. The top images show first micrographs, the middle images 6th micrographs of image series. Bottom left: Micrograph taken after recording both series of stereo images at the initial tilt position, corresponding to micrographs left above. Bottom right: Regions of interest marked for discussion (chapter 6.5.1). Micrograph identical to the one shown middle left. 
the strongly absorbing small particle on the right side of the assembly. Although its position remains stable from the last sequence of the first series to the first image of the second series, a difference of $130 \mathrm{~nm}$ exists in the control image. This motion occurred within the second image series, where the position changes by $130 \mathrm{~nm}$ downwards. The $\mathrm{x}$-values vary between the last image of the first image series and the control image just insignificantly, apart from the small singular particle on the left side. This particle was not visible as singular particle in the first image series, but under the tilted position of the right micrographs, a distance to the main cluster becomes visible. This supports the conclusion of spatial depth, supported by the stability of the particle during the second image series. But the detected drift may had happened during the 12 min time slot of tilting the sample for recording the stereo images, which is even more likely as during the tilt between the stereo image series and the control micrograph, because the shape of the structures resembles more within the second image series and the control micrograph, and seems to be still in the formation process at least until finishing the record of the first image series. The main drift, referring to the sinking of the whole assembly, can be determined to a maximum of the $12 \mathrm{~min}$ time period of the first image series. The drift caused a blurred image of this area in the first image series. Based on this conclusion, stereo analysis of this area can be carried out combining the second tilt series and the control micrograph, with limitations due to the low resolution of the control image. The small particle on the right has to be excluded because of its motion during the tilt series.

Region number 2 indicates a detached cluster of three connected particles in a distance measured in both the projection images of $1.8 \mu \mathrm{m}$ and $1.9 \mu \mathrm{m}$ to the assembly of region number 2. All particles remain without any drift or change. The right cusp and the bottom particle do not show a parallax within the stereo micrographs, so they are situated at equal z-positions. But the distance of the right cusp and left cusp of the assembly changes by $180 \mathrm{~nm}$, which is an indication for spatial extension at the intermediate tilt position, as described in chapter 3.2.1. By xstereo processing (not presented here), a reconstruction of the spatial coordinates develops a distance of $300 \mathrm{~nm}$ in z-direction between these two cusps. This is the depth of region number 2 , not taking into account the spatial extension of the particular particles.

The particles in region number 3 remain also stable during the stereo experiment as found by comparing the images of both image series and the first image series with the control micrograph. Thus, the variation of parallax clearly observable within the stereo images implies spatial depth. By xstereo analysis (figures not presented), the 
top and bottom cusp of the right particle result to have a distance in z-direction of about $1.9 \mu \mathrm{m}$. Both particles attached on the right side of the central cluster of region 3 lie in one plane, but in $4 \mu \mathrm{m}$ distance in z-direction to the upper cusp of the right particle. The evident differences in foci verify this notable depth. To depict the spatial position of edges and small particles, this region is very well suited for detailed analysis with xstereo.

Another area of stable particles is defined by number 4, where a cluster of weakly absorbing particles seems to be attached to the strongly absorbing particle in the middle of the image in the first image series, but is revealed to have some distance in the second image series. The spatial depth of the cluster is at least $500 \mathrm{~nm}$, considering the particle top right and the bottom cusp of cluster.

A singular platelet is marked as region 5. There is no detectable drift, but a clear decrease in extension in $\mathrm{x}$-direction in the stereo images, measured to be around $250 \mathrm{~nm}$. It was reconstructed to a depth of $850 \mathrm{~nm}$, which is approximately a quarter of the platelet length in the projections and means a notable incline of the platelet relative to the $\mathrm{Si}_{3} \mathrm{~N}_{4}$ membranes.

Two particles extending into the image field in region 6 drift upwards and to the left during the first image series, whereby the upper particle disappears. The lower particle maintains its position reached at the end of the first image series, as indicated by the control micrograph. The fact that the $\mathrm{x}$ and $\mathrm{y}$ coordinates do not change in the tilted stereo image leads to the assumption that the particle became attached to one of the $\mathrm{Si}_{3} \mathrm{~N}_{4}$ membranes.

Region 7 includes a small cluster of weakly absorbing particles in a distance in the projection image of about $130 \mathrm{~nm}$ to a large, strongly absorbing particle. The distances of the cluster edges to the edge of the large particle do not change neither within the image series nor by tilting the object. But the whole assembly shows a shift to the right within the first image series and in comparison with the control image. The shift between the last sequence of the first image series and the control image is just slightly larger than the shift within the first image series, i. e. approximately $150 \mathrm{~nm}$ compared to $200 \mathrm{~nm}$. Additionally, within the first image series, a upward shift of about $130 \mathrm{~nm}$ occurred, but the thereby reached y-values stayed stable afterwards. Based on these observations, the conclusion can be made that the particles are all situated in a layer very close to one $\mathrm{Si}_{3} \mathrm{~N}_{4}$ membrane, similar to the particle of region number 6 , and that the shift occurred in the 24 min time period of recording the first image series and tilting the object. 
In region 8 , another cluster of small, weakly absorbing particles is selected. The cluster appears very blurred in the first image series, but focused in the second series as well as in the control image, revealing its consistence of small particles. Because of the blurriness in the first image stack, it is difficult to measure particular edges or relevant structures, but a mayor drift of the assembly can be excluded. Also within the second tilt series, the particles remain stable. Therefor, the blurriness can be referred to a defocus. The z-position of this assembly must be equal to the z-position of the small particles in the left side of region 3, which are of similar shape and absorption and appear also blurred only in the first image series. As the particles in region 7 , these particles are probably attached to one of the $\mathrm{Si}_{3} \mathrm{~N}_{4}$ membrane, but here it appears to be the opposite $\mathrm{Si}_{3} \mathrm{~N}_{4}$ membrane situated more out of focus.

Region 9 is another example for motion probably occurred during the first image series and the tilt, because a shift is detected during the first image series, and between the last image of the first series and the control image, but not during the second image series. Thus, the spatial distance between both large particles shown in this region cannot be determined by the series of stereo images.

The detailed discussion of this stereo experiment demonstrates the variety of possible dynamical changes within an aqueous colloidal sample (see also figure 6.32 with corresponding discussion in the chapter 6.6). With the actual experimental setup, spatial arrangements can be determined for static assemblies shown in regions 2 to 5 . With adjustment to the all-over drift, region number 7 allows for stereo analysis within this restricted region. But as the particle movements, as detected of region number 7 relative to the entire sample area and within region number 9 , as well as the particle formations, as seen in region number 1 , are hereby proven to be slow in relation to reachable exposure times for high resolution X-ray micrographs. Thus, these movements can be determined in three dimensions by X-ray stereo microscopy as discussed in chapter 7 .

An overview of the above discussed spatial depth of the aqueous montmorillonite sample presented in figures 6.21 and 6.22 is visualised by xstereo processing (figure 6.23). On the left, a detail of the left image shown in figure 6.21 and turned by $90^{\circ}$ is presented. Representative structures of the motionless regions number 2 to 5 (figure 6.22 bottom right) are marked for xstereo processing. The z-positions of the marked structures are depicted to merge the spatial information discussed separately for the particular regions. The plot of the marked structures is shown in figure 6.23 right. The vertical axis corresponds to the depth of the stereo images (z-axis). The unit is pixel, where $100 \mathrm{pxl}$ correspond to $2.56 \mu \mathrm{m}$. The plot demonstrates that the particles of the 

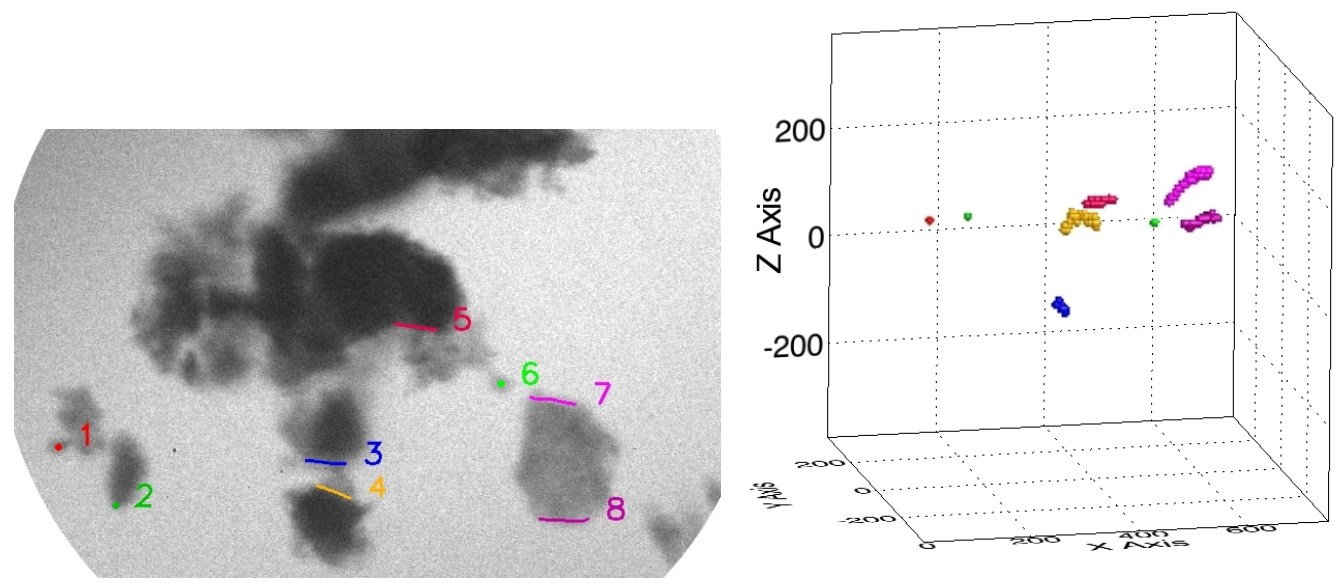

Fig. 6.23: xstereo processing of the aqueous sample of montmorillonite particles with carbon nanotubes (baytubes) as figure 6.21 to visualise the spatial depth. Left: Detail of the left image shown in figure 6.21 and turned by $90^{\circ}$. Representative structures marked for xstereo processing. Right: View of the plot presenting the marked structures in three dimensions. The vertical axis corresponds to the depth of the stereo images (z-axis). The unit is pixel, $100 \mathrm{pxl}$ correspond to $2.56 \mu \mathrm{m}$.

regions 2 and 4 (marked as structures 1,2 and 5,6) lie in about the same z-plane. As already mentioned, the platelet of region number 5 shows an incline, with the lower edge, marked as line 8 , within the same z-plane as the structures of regions number 2 and 4, and with the upper edge, marked as line 7, above it. The spatial depth within region number 3 is clearly shown by the lines 3 and 4 , representing neighbouring edges of two particles. While the particles shown in the bottom of the micrograph (structure 4) are situated in about the same z-plane as the other structures, the edge of the particle in the middle of region 3 , here marked as line 3 , lies in notable distance of $8 \mu \mathrm{m}$ below. The symmetry of the particle distribution with z-values between $+4.1 \mu \mathrm{m}$ and $-4 \mu \mathrm{m}$, visible in the plot as distribution between the corresponding pixel values of \pm 156 along the vertical axis, leads to the conclusion that the centre z-plane of the membrane stack matches well with the centre plane $(\mathrm{z}=0)$ of the plot.

Another region of the same sample is demonstrated in figure 6.24 with a time lag of $1.5 \mathrm{hrs}$ to the previous experiment (figure 6.21). The sample was still aqueous, but without motion during this experiment as ascertained by the same method of imaging series as above. Each stereo image is a sum of 5 micrographs taken with 60 s exposure time each. The first image series (stereo image shown in figure 6.24 left) was acquired within $8 \mathrm{~min}$. Other $8 \mathrm{~min}$ later, the second series (stereo image shown in figure 6.24 

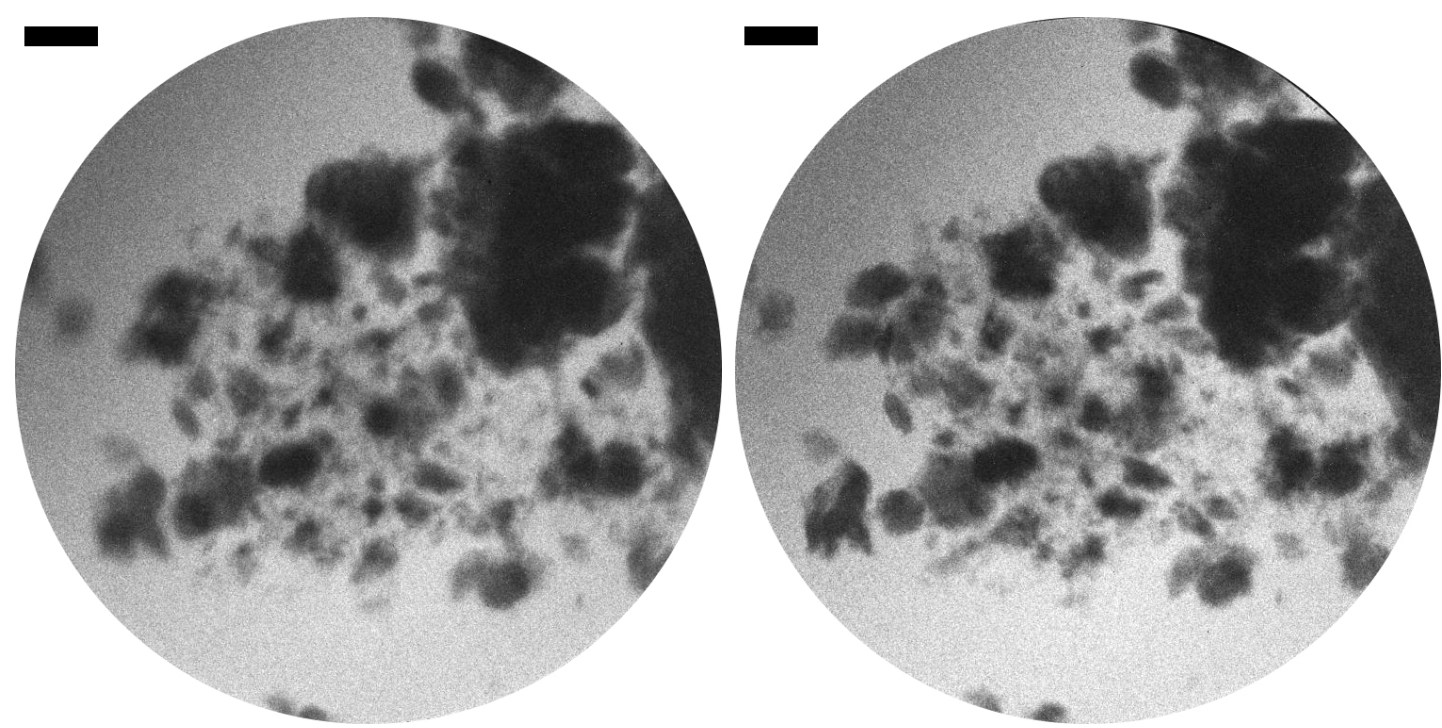

Fig. 6.24: Aqueous sample of montmorillonite particles with carbon nanotubes (baytubes) as figure 6.21 , but showing another sample region. The tilt angle is $\Delta \theta=14^{\circ}$ around the vertical image axis. Each image is a sum of 6 micrographs taken with $60 \mathrm{~s}$ exposure time, respectively. Images taken with the CSXM at $E=368 \mathrm{eV}$. The scale bars indicate $2 \mu \mathrm{m}$.

right) was recorded within $11 \mathrm{~min}$. The sample region presented here includes a large assembly of particles with different sizes in a higher density and larger extension than the region imaged before.

The depth of this sample region is also depicted by xstereo processing and presented in figure 6.25. On the left, a detail of the first stereo image (figure 6.24 left) is shown in a position turned by $90^{\circ}$, so the stereo tilt axis is parallel to the horizontal image axis. Some structures are marked and their spatial distribution is presented in a plot shown on the right. The vertical axis of the plot corresponds to the depth of the sample. The unit is pixel, where $100 \mathrm{pxl}$ correspond to $2.56 \mu \mathrm{m}$. The maximum depth detected is $4 \mu \mathrm{m}$, which is the distance between the edges marked as lines 6 and 7 . This reveals a notable distance in z-direction between the neighbouring structures of the bottom right corner of the detail image (bottom left in the stereo images of figure 6.24), which is not visible in the projection images. An intermediate z-position is revealed for the large particle shown in the upper left corner of the detail image, of which two edges are marked as lines 1 and 2 and a cusp as point 3. This result has to be scrutinised as the particle is probably a spatially extended particle. In case of a clay platelet, the selected edges can be assumed to be at a height of one basal plane. But in case of a soil particle 

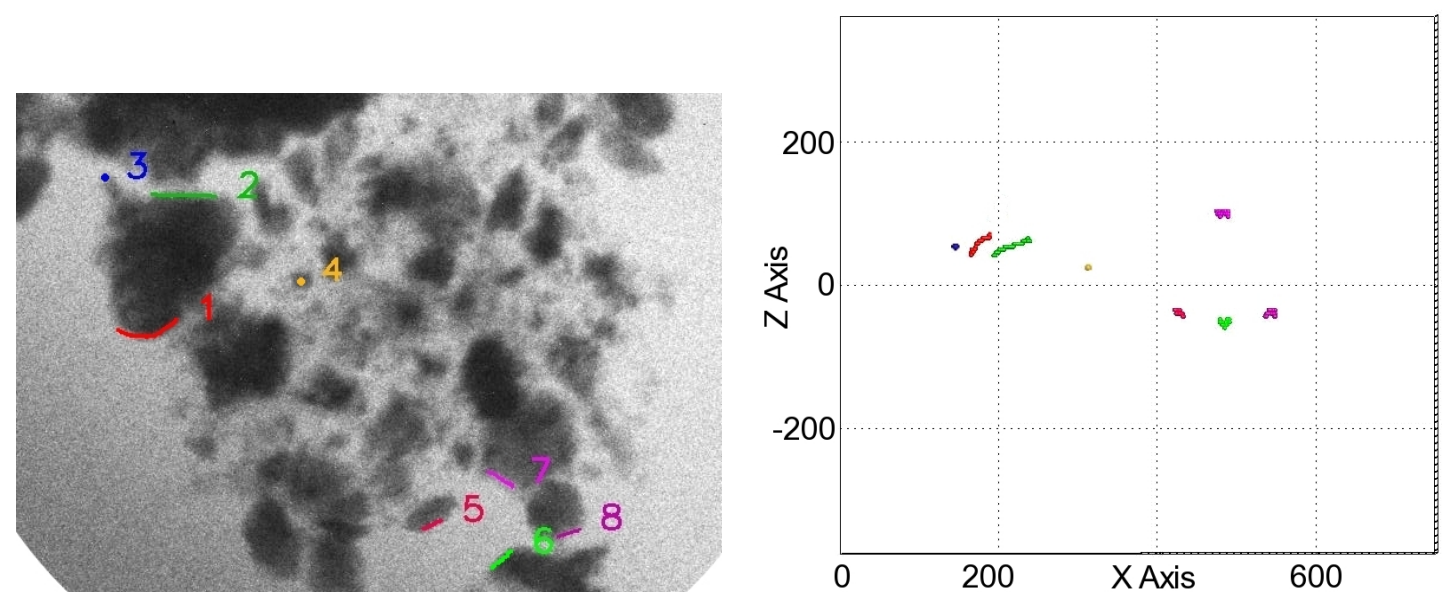

Fig. 6.25: xstereo processing of the aqueous sample of montmorillonite particles with carbon nanotubes (baytubes) as figure 6.21 to visualise the spatial depth. Left: Detail of the left image shown in figure 6.24 and turned by $90^{\circ}$. Some structures are marked for xstereo processing. Right: View of the plot presenting the marked structures in three dimensions. The vertical axis corresponds to the depth of the sample (z-axis). The unit is pixel, $100 \mathrm{pxl}$ correspond to $2.56 \mu \mathrm{m}$.

of arbitrary shape, the height of its edges in the projection images can be at any z-value covered by the particle volume.

Conflating the results of both stereo experiments shown in figures 6.21 and 6.24, the maintenance of depth of this aqueous sample even after several hours within a sealed stack of two $\mathrm{Si}_{3} \mathrm{~N}_{4}$ membranes is demonstrated, as well as a reduction of particle motion within one hour after preparing the sample.

However, a distinction between clay particles and carbon nanotubes on behalf of the shape is not possible due to the occurrence of small montmorillonite particles as discussed previously in chapters 6.5 and 6.5.1. Therefore, further experiments were performed at the STXM using elemental contrast at the carbon absorption edge. Utilising the same stock solutions as for the above presented experiments performed at the CSXM, dispersed carbon nanotubes (baytubes) were added to aqueous montmorillonite. One drop of this mixture was applied onto a $\mathrm{Si}_{3} \mathrm{~N}_{4}$ membrane and let dry. Figure 6.26 shows two micrographs taken of a dried mixture of montmorillonite and carbon nanotubes (baytubes) with the STXM at $280 \mathrm{eV}$ (figure 6.26 left) and $300 \mathrm{eV}$ (figure 6.26 right). The exposure time was $15 \mathrm{~ms}$ per pixel and the recorded image size $12 \mathrm{x} 15 \mu \mathrm{m}^{2}$ on $240 \times 300 \mathrm{pxl}^{2}$ whereupon an image size of $275 \times 215 \mathrm{pxl}^{2}$ is presented. The scale bars 

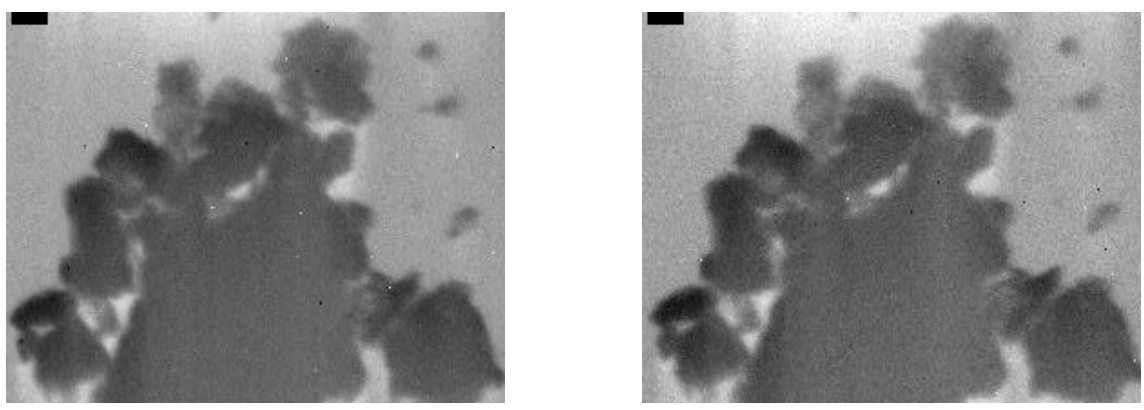

Fig. 6.26: Elemental mapping of a montmorillonite dispersion mixed with carbon nanotubes (baytubes) and let dry onto a $\mathrm{Si}_{3} \mathrm{~N}_{4}$ membrane. Micrographs taken under two different energies: $280 \mathrm{eV}$ (left) and $300 \mathrm{eV}$ (right). No carbon detected. The exposure time was $15 \mathrm{~ms}$ per pixel, the recorded image size $12 \times 15 \mu^{2}$ on $240 \times 300 \mathrm{pxl}^{2}$ (presented as $275 \times 215 \mathrm{pxl}^{2}$ ). Images taken with the STXM. The scale bars indicate $1 \mu \mathrm{m}$.

indicate $1 \mu \mathrm{m}$. As both micrographs depict similar absorption contrast, this elemental mapping of carbon proves that carbon nanotubes are lacking in the imaged sample area.

In contrast, another experiment performed similarly with the STXM of the same sample as above, but of a different sample area, reveals an extended deposit of carbon nanotubes attached to smaller clay platelets (figure 6.27). The left micrograph presented in figure 6.27 gives an overview of the investigated sample area of $90 \mathrm{x} 90 \mu \mathrm{m}^{2}$ on $100 \times 100 \mathrm{pxl}^{2}$. The image was taken at $300 \mathrm{eV}$ with an exposure time of $9 \mathrm{~ms}$ per pixel. Uniformly strongly absorbing contiguous clusters appear in the upper part of the image as well as in the downmost image part. In the bottom left image corner and in the middle of the image clusters with smaller structures and varying absorption contrast are visible. The micrographs presented in the middle and right of figure 6.27 show a detail of the cluster from the centre of the overview image with an image size of $10 \times 20 \mu \mathrm{m}^{2}$ on $200 \times 400 \mathrm{pix}^{2}$. The exposure time was also $9 \mathrm{~ms}$ per pixel, and the scale bars indicate $1 \mu \mathrm{m}$. The images were taken at $280 \mathrm{eV}$ (figure 6.27 middle) and $300 \mathrm{eV}$ (figure 6.27 right). By the element contrast, carbon nanotubes are identified as extended deposits between clusters of clay platelets. Although the shape of the carbon nanotubes is similar to the shape of the pure carbon nanotubes (baytubes) shown in figure 6.5, it is not possible to distinguish them from the clay platelets as some look alike. The impression that most of the areas with carbon nanotubes has a lower contrast in the image taken at $300 \mathrm{eV}$ is misleading and due to a lower thickness compared to most clay particles. Rather, clay particles of low contrast are visible also in the image taken at $280 \mathrm{eV}$, and 

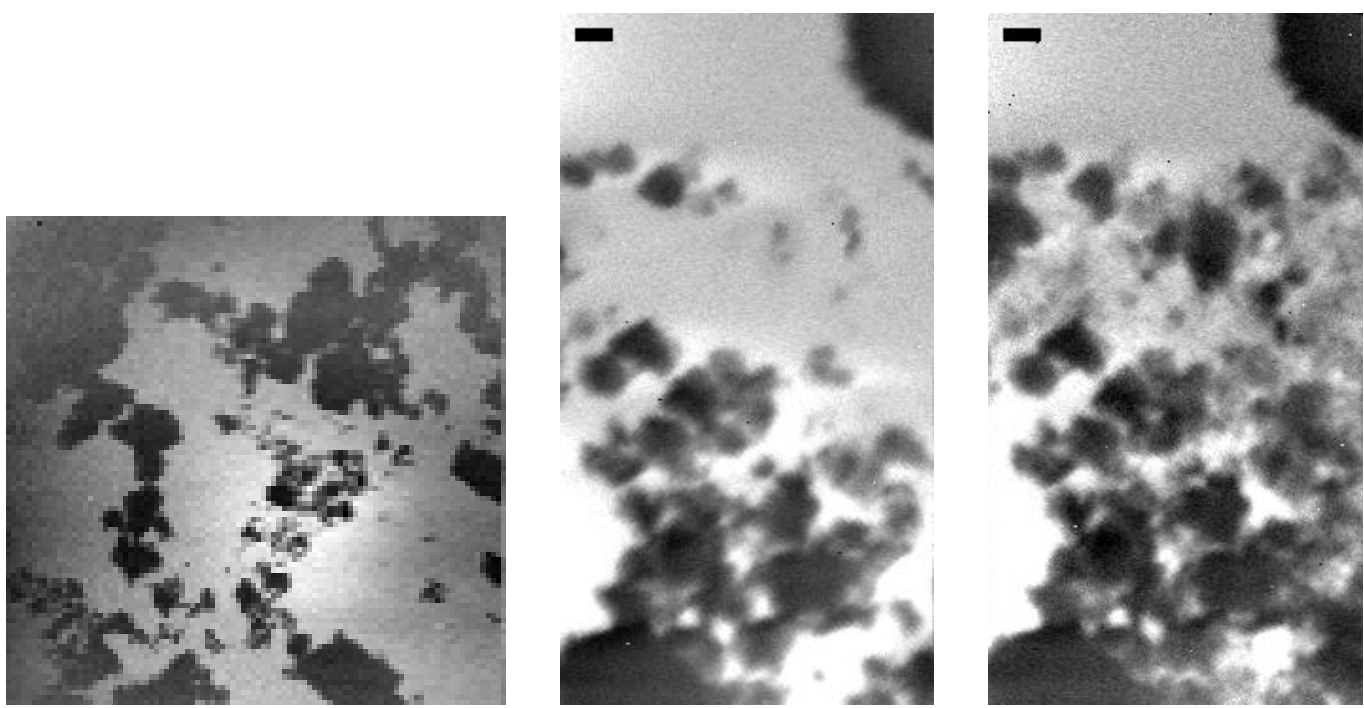

Fig. 6.27: Same sample as shown in figure 6.26, but area containing carbon nanotubes (baytubes). Left: Overview micrograph of a montmorillonite dispersion mixed with carbon nanotubes and let dry onto a $\mathrm{Si}_{3} \mathrm{~N}_{4}$ membrane. The image was taken at $300 \mathrm{eV}$. The exposure time was $9 \mathrm{~ms}$ per pixel, the image size $90 \times 90 \mu \mathrm{m}^{2}$ on $100 \times 100 \mathrm{pxl}{ }^{2}$. Middle and right: Elemental mapping of carbon from a detail of the centre part of the sample area shown on the left. Images taken at $280 \mathrm{eV}$ (middle) and $300 \mathrm{eV}$ (right). Structures appearing just in the right image can be identified as carbon nanotubes. The exposure time was $9 \mathrm{~ms}$, the image size $10 \times 20 \mu \mathrm{m}^{2}$ on $200 \times 400 \mathrm{pix}^{2}\left(198 \times 366 \mathrm{pix}^{2}\right.$ presented). The scale bar indicates $1 \mu \mathrm{m}$. Images taken with the STXM.

strongly absorbing clusters of carbon nanotubes are only present in the image taken at $300 \mathrm{eV}$. Thus, a secure differentiation is just possible by elemental mapping.

The irregular deposit of carbon nanotubes (baytubes) in mixtures with montmorillonite developed by different results from one sample as elaborated from figures 6.26 and 6.27 impedes additionally the evaluation with regard to the identification of carbon nanotubes in the micrographs presented in figures 6.21 and 6.24.

Also carbon nanotubes (rCNT) described in chapter 5.5.2 and presented in chapter 6.2, figure 6.6, were added to aqueous montmorillonite and imaged in the dried state with the STXM. Elemental mapping of the rCNTs is presented in figure 6.28. The recorded image size is $20 \times 10 \mu \mathrm{m}^{2}$ on $400 \times 200 \mathrm{pxl}^{2}$, whereupon cutouts of $390 \times 170 \mathrm{pxl}^{2}$ are presented. The exposure time was $10 \mathrm{~ms}$ per pixel. The scale bars indicate $1 \mu \mathrm{m}$. The left micrograph was taken below the carbon absorption edge at $282 \mathrm{eV}$, and the right micrograph above the absorption edge at $314 \mathrm{eV}$. In the left image, an area completely coated with particles of different size and absorption contrast extends from the 

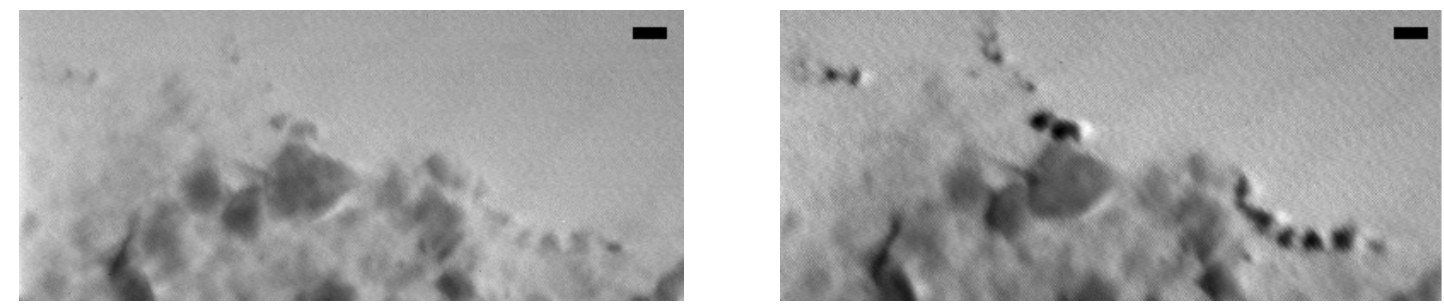

Fig. 6.28: Micrographs of a montmorillonite sample mixed with carbon nanotubes (rCNT) and dried onto a $\mathrm{Si}_{3} \mathrm{~N}_{4}$ membrane. Images taken at $282 \mathrm{eV}$ (left) and $314 \mathrm{eV}$ (right) for elemental mapping of carbon. The recorded image size is $20 \times 10 \mu \mathrm{m}^{2}$ on $400 \times 200 \mathrm{px} \mathrm{l}^{2}$ (390 $170 \mathrm{pxl}^{2}$ presented), the exposure time $10 \mathrm{~ms}$ per pixel. Images taken with the STXM. The scale bars indicate $1 \mu \mathrm{m}$.

bottom into the image. Comparing both images, the provided elemental contrast reveals particular clusters of carbon nanotubes situated only on the outermost border of the clay area. Thus, the assembly within the dried sample differs from the one of CNT and montmorillonite shown in figure 6.27. As true for the above presented montmorillonite samples mixed with carbon nanotubes (baytubes), it is not possible to identify carbon nanotubes without elemental mapping, although a strong contrast of the clusters of carbon nanotubes as shown in figure 6.6 provides an indication for carbon nanotubes.

To reveal the dynamical characteristics of the mixture of montmorillonite with carbon nanotubes, the addition of carbon nanotubes ( $\mathrm{rCNT}$ ) to a montmorillonite dispersion was performed with the STXM using a capillary as sample holder. Two pairs of images were taken of assumingly the same sample region with equal image parameters, one before (figure 6.29 left) and one after (figure 6.29 middle) injecting additional carbon nanotubes $(\mathrm{rCNT})$. The pair of stereo images shown on the right side is taken parallel with the stereo pair shown in the middle, but picturing a sample region towards the capillary tip. All image pairs were taken at $400 \mathrm{eV}$ within $30 \mathrm{~min}$, respectively, of a sample region of $8 \times 7 \mu \mathrm{m}^{2}$ on $160 \times 140 \mathrm{pxl}^{2}$ with $24 \mathrm{~ms}$ exposure time per pixel. The tilt angle around the horizontal capillary axis is approximately $15^{\circ}$. The time between the pairs was $5 \mathrm{hrs}$, resulting in a big change in structure. With the light microsope, the initial cluster was identified after the addition of carbon nanotubes as the cluster shown in the middle image pair, although it was situated slightly drifted towards the capillary tip. This is recognisable in the decrease of capillary diameter barely visible in the X-ray micrographs. As elemental mapping of carbon was not possible for this setup (chapter 4.2.1), but rather an imaging energy of $400 \mathrm{eV}$ had to be used, identification of carbon 

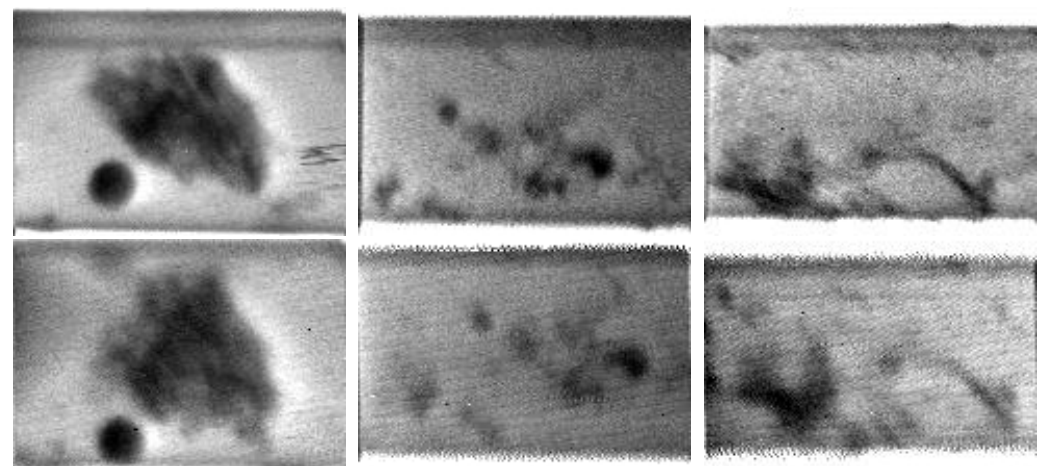

Fig. 6.29: Three pairs of stereo micrographs of a dispersion of montmorillonite in a capillary tip, revealing structural changes after the addition of carbon nanotubes. Left: Stereo image pair of pure dispersion of montmorillonite. Middle: Same sample position after the addition of carbon nanotubes (rCNT) approximately 5 hours before imaging. Right: Same sample as besides, but different sample region. All images taken at $E=400 \mathrm{eV}$ at the STXM. The recorded image size is $8 \times 7 \mu \mathrm{m}$ on $160 \times 140 \mathrm{pxl} \mathrm{l}^{2}$ with $24 \mathrm{~ms}$ exposure time per pixel.

nanotubes is not possible. The increased absorption of water at $400 \mathrm{eV}$ compared to $300 \mathrm{eV}$ degrades the contrast of the necessarily aqueous sample.

\subsubsection{Montmorillonite with detergent}

Structural changes of dispersed montmorillonite were evoked by the addition of a $1 \%$ dispersion of the cationic detergent DTB (dodecyltrimethylammoniumbromide). As discussed in chapter 5.5.1, DTB acts as an coagulating agent when added to dispersed colloidal soil particles. In figure 6.30 left, two micrographs of aqueous montmorillonite mixed with DTB between two $\mathrm{Si}_{3} \mathrm{~N}_{4}$ membranes are presented. Both images were taken with the CSXM at $368 \mathrm{eV}$ and show sample areas of approximately $20 \mu \mathrm{m}$ diameter, the scale bars indicate $2 \mu \mathrm{m}$. Each micrograph is formed as the sum of 10 single micrographs taken with an exposure time of $60 \mathrm{~s}$, respectively, to exclude or identify particle movements during the total exposure time as reasons for blurred structures in the micrographs. The micrograph top left of figure 6.30 shows a montmorillonite sample mixed with detergent in a ratio of $1: 4$. In the centre of the image, a lateral extended cluster of clay platelets is located surrounded by densely distributed smaller clay platelets of poor contrast. While the central cluster appears in focus, most of the surrounding structures are blurred. The micrograph is part of a stereo pair, of which the second one is not presented. Some relevant structures recognisable in both stereo images are marked for 

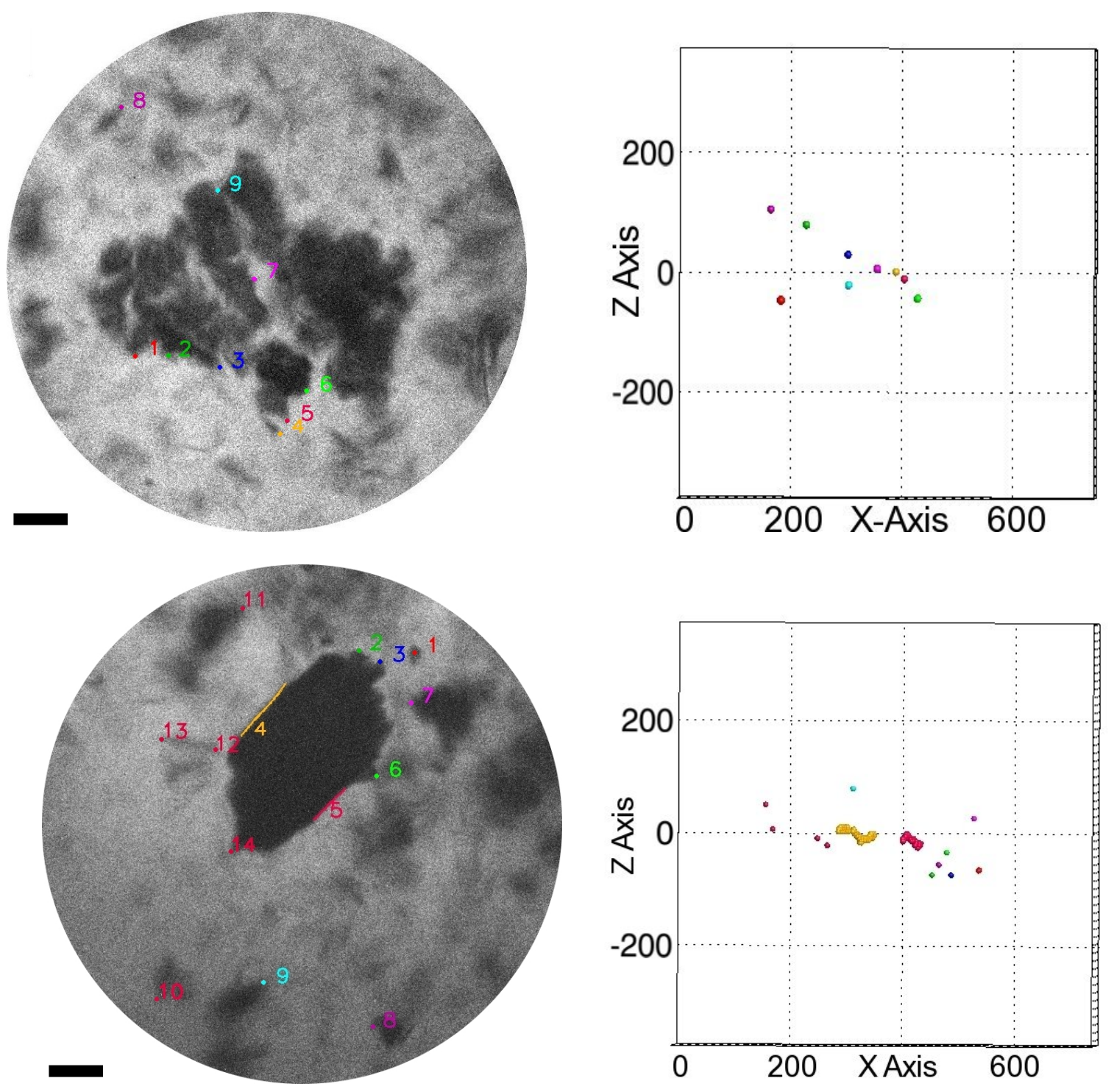

Fig. 6.30: Micrographs of an aqueous montmorillonite sample mixed with detergent between two $\mathrm{Si}_{3} \mathrm{~N}_{4}$ membranes. Left: The mixing ratios were montmorillonite to detergent 4:1 (top left) and 2:1 (bottom left). Each micrograph is an addition of 10 single micrographs taken with an exposure time of $60 \mathrm{~s}$, respectively. Images taken with the CSXM at $E=368 \mathrm{eV}$. The scale bars indicate $2 \mu \mathrm{m}$. Right: Views of the $3 d$ plots of the marked structures created with xstereo, depicting the sample depths. The unit is pixel, $100 \mathrm{pxl}$ correspond to $2.56 \mu \mathrm{m}$. 
xstereo processing. The difficulty in identifying matching points of structures with this sample is due to the compact structures and the low resolution. However, some cusps could be selected. A view of the created three-dimensional plot of the marked structures is shown in figure 6.30 top right, depicting the sample depth as vertical axis and the horizontal axis analogue to the corresponding micrograph. The unit is pixel, where $100 \mathrm{pxl}$ correspond to $2.56 \mu \mathrm{m}$.

A sample of higher content of detergent is shown in figure 6.30 bottom left. The ratio of montmorillonite to detergent is 1:2. One large clay particle of more than $6 \mu \mathrm{m}$ length and $4 \mu \mathrm{m}$ width is visible in the upper image centre. Furthermore, blurred structures are distributed as in the image above, but less dense. Also this micrograph is part of a stereo pair, of which the second micrograph is not shown. Structures are marked with xstereo and the created three-dimensional plot is depicted as above with the depth as vertical axis and the horizontal axis similar to the one of the respective micrograph. The unit is pixel, where $100 \mathrm{pxl}$ correspond to $2.56 \mu \mathrm{m}$.

Comparing both micrographs, the expectation of denser clusters in the sample with higher detergent content may be supported, but not as clearly as desired. Also the results from the xstereo processing reveal depth of about $3.8 \mu \mathrm{m}$ for both samples. However, the potential of investigating the influence of detergent percentage to the coagulation of dispersed colloids can be utilised with improved image quality and then even in combination with instantaneous mixing (chapter 7).

\subsection{Calcaric phaeozem}

As one example for an naturally occurring soil, calcaric phaeozem (German: GleySchwarzerde / Gley-Tschernosem) had been chosen (chapter 5.4). Figure 6.31 shows two micrographs of chernozem dried onto a $\mathrm{Si}_{3} \mathrm{~N}_{4}$ membrane and taken with the TwinMic at $700 \mathrm{eV}$ (figure 6.31 left) and $708 \mathrm{eV}$ (figure 6.31 right) below and above the absorption edge of iron. The exposure time was $70 \mathrm{~ms}$ per pixel, and the image size is $20 \mathrm{x} 20 \mu \mathrm{m}^{2}$ on $100 \times 100 \mathrm{pxl}^{2}$. A large cluster of chernozem particles is extended from the left bottom into the image field. In the image taken below the iron absorption edge (figure 6.31 left), contrast differences between individual cluster particles are clearly visible. Most particles of low contrast switch to high contrast in the micrograph taken above the iron absorption edge (figure 6.31 right), for example in the peak of the cluster shown in the top right image region. Thus, iron containing soil particles are identified.

Phaeozem in an aqueous state between two $\mathrm{Si}_{3} \mathrm{~N}_{4}$ membranes was examined with the CSXM. Figure 6.32 presents 9 micrographs taken at $368 \mathrm{eV}$ with $60 \mathrm{~s}$ exposure time 

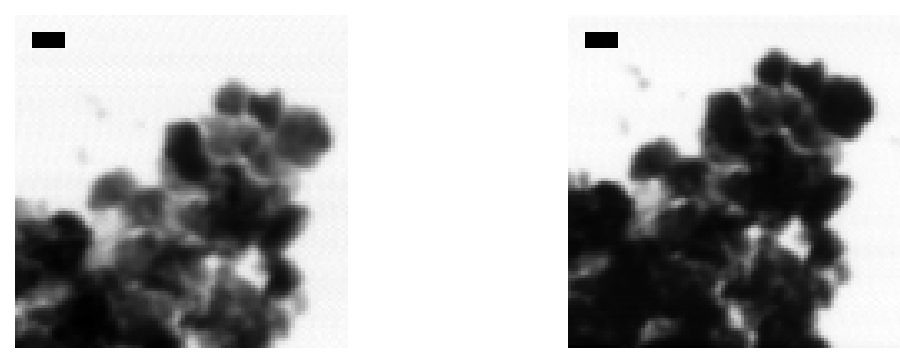

Fig. 6.31: Micrographs of chernozem dried onto a $\mathrm{Si}_{3} N_{4}$ membrane. Images taken at $700 \mathrm{eV}$ (left) and $708 \mathrm{eV}$ (right). The exposure time was $70 \mathrm{~ms}$ per pixel, the image size is $20 \times 20 \mu \mathrm{m}^{2}$ on $100 \times 100 \mathrm{pxl}^{2}$. Images taken with the TwinMic. The scale bars indicate $2 \mu \mathrm{m}$.

under constant focus adjustment. The micrographs were recorded in direct succession, the order from image a to i corresponds to the chronological order. Clusters of different shape and absorption are visible. The thickness of the sample is proven by the varying sharpness of the particles. Another prove of the existing water layer is the detection of sample movements. Five regions of interest are indicated by the numbers numbers $1-5$ in micrograph a of figure 6.32. In the regions on the right side of each number, structural changes are visible over the image sequences. In region number 1 , a conglomeration of smaller particles changes its formation slightly over the first images (a to d), changing positions of small components within and on the bottom border of the cluster, and increasing the distance to the cluster situated right to it. The achieved position stays stable over the following micrographs ( $\mathrm{d}$ to i). Right besides the number 2, a single particle is pointed out which moves initially slowly towards a large particle beneath it (a to c), but than changes the direction towards smaller particles situated above (c to g). Apparently, it gets attached to three small platelets in a position stable in the following (g to i). With number 3, two large platelets are marked which initially approximate (a to c), remain stable (c to h), and finally remove slightly (h to i). Next to number 4, an interaction is demonstrated between two particles in the range of $500 \mathrm{~nm}$, one with low contrast on the right and one with high contrast. The particle of low contrast removes slightly from the particle of high contrast (a to c), possibly remaining in contact by a linear structure of low contrast. By this movement, it gets into the focus and is imaged with improved sharpness. At the same time, the particle of high contrast moves slightly up the border of the large particle to which it seems to be attached. Another small structure appears above the particle of high contrast and tilts towards the low contrast particle (c to f), while the high contrast particle moves back towards the pike of the 

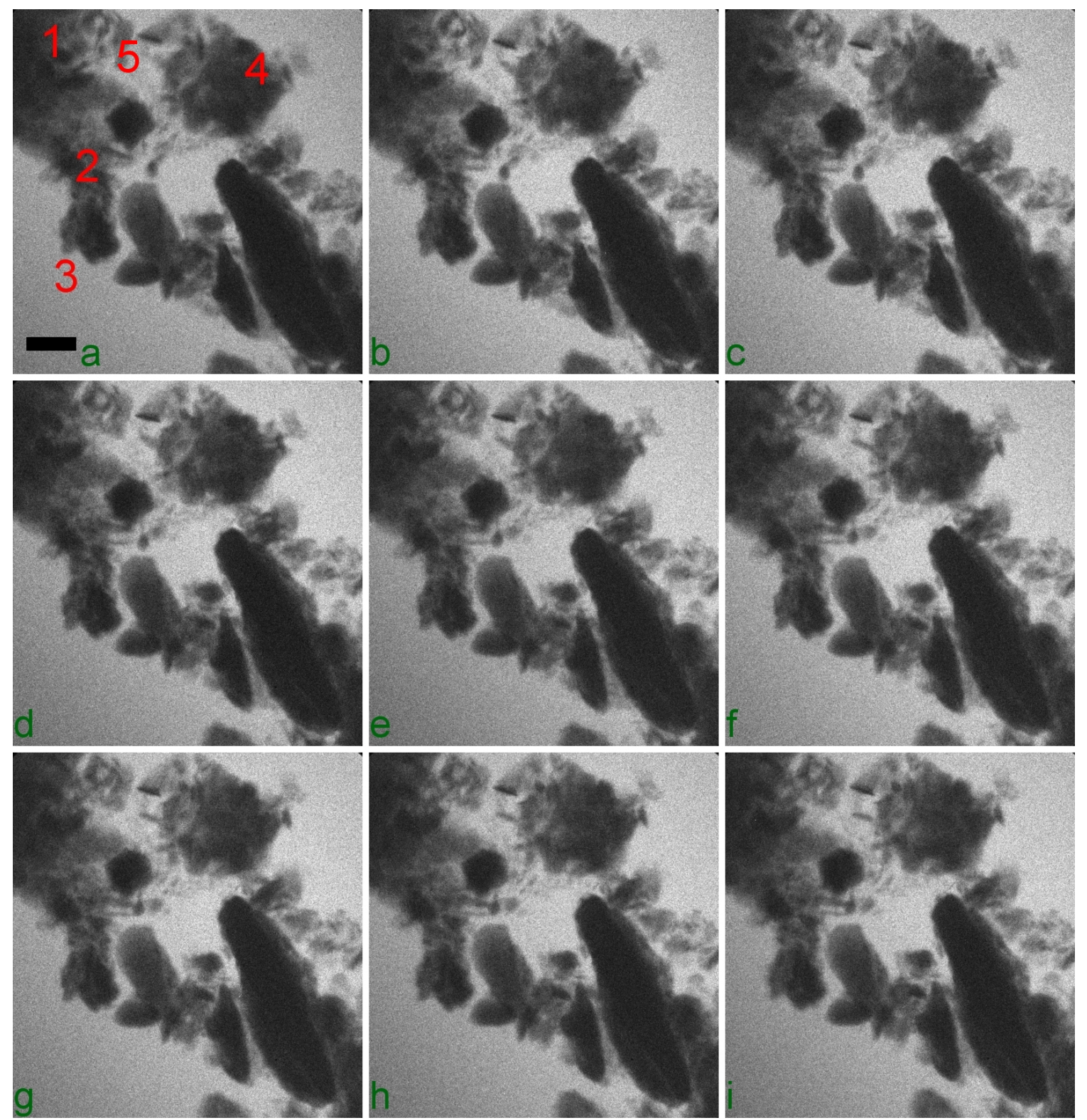

Fig. 6.32: Nine micrographs of an aqueous phaeozem sample between two $\mathrm{Si}_{3} \mathrm{~N}_{4}$ membranes taken in direct succession, starting from a to $i$. In the regions on the right side of the numbers 1- 5 (marked in micrograph a), structural changes are visible over the image sequences. The exposure time was $60 \mathrm{~s}$ per image. Images taken with the CSXM at $E=368 \mathrm{eV}$. The scale bar indicates $2 \mu \mathrm{m}$. 

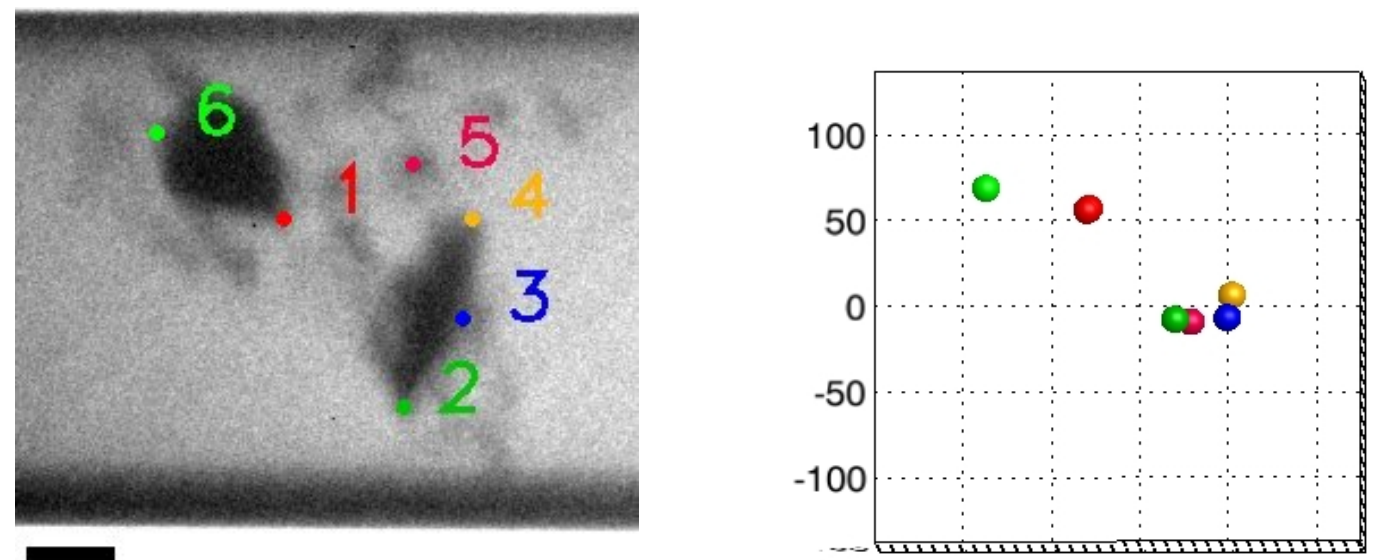

Fig. 6.33: xstereo processing of a calcaric phaeozem dispersion in a capillary of $6 \mu \mathrm{m}$ diameter. Left: One micrograph of a stereo pair. Some structures marked for stereo analysis. The image is a sum of two micrographs with 300 seconds exposure time each. Images taken with the CSXM at $E=368 \mathrm{eV}$. The scale bar indicates $1 \mu \mathrm{m}$. Right: View of plot depicting the spatial distribution of the marked structures. The unit is pixel, $100 \mathrm{pxl}$ correspond to $1.28 \mu \mathrm{m}$.

large particle. In the last sequences ( $\mathrm{g}$ to $\mathrm{f}$ ), the particle of low contrast again looses slightly in contrast, and the high contrast particle moves very little up again. Beside number 5, a structure attached to a big cluster develops from a barely visible particle (a) to a linear structure(b to f). It may be a platelet which tilts with its basal plane towards a position parallel to the X-ray light. However, other particles remain stable, for example the large particle situated in the bottom right region of the image. As the most spacious component of the whole conglomeration, it is probably stuck between the $\mathrm{Si}_{3} \mathrm{~N}_{4}$ membranes and determines and maintains their distance.

It is noticeable that the detected movements are so slow, that they are just visible comparing several micrograph sequences, and that the particles may appear sharply, as the scale of movement during the exposure time of $60 \mathrm{~s}$ does not exceed the resolution of the image. Yet, to take high contrast images of aqueous samples, the reduction of exposure time is necessary and will be achieved in the future with the implementation of a new laser system to the CSXM (chapter 4.1.2).

The ability of the CSXM to image aqueous samples within a capillary is proven by first images as presented in figure 6.33. On the left, one micrograph of a stereo pair of a calcaric phaeozem dispersion in a capillary tip of approximately $6 \mu \mathrm{m}$ diameter is shown. The image is a sum of two micrographs with 300 seconds exposure time each, taken at $\mathrm{E}=368 \mathrm{eV}$ with the CSXM. The scale bar indicates $1 \mu \mathrm{m}$. A cluster of phaeozem is 
visible containing particles of different sizes. Some identification points, recognisable in both stereo images, are marked and processed with xstereo. A view of the resulting plot depicting the spatial distribution is shown in figure 6.33 right. The vertical axis of the plot corresponds to the depth of the sample, the horizontal axis is analogue to the horizontal axis of the micrograph. The different z-positions of both large particles is determined by the spatial positions of their cusps marked as dots 1 and 6 (left particle) and 2 to 4 (right particle). However, the thickness of the particles cannot be determined, thus the shortest distance between the surfaces of both particles is not revealed. The small particle of which the centre is marked as dot 5 lies at the same z-plane as the cusps of the right particle.

For the xstereo processing, a tilt angle around the vertical image axis of $30^{\circ}$ is assumed. But as the experimental setup did not provide a tilt device, the capillary was tilted manually for this first experiment at the CSXM of tilting a capillary. The estimated accuracy of the tilt angle $\theta$ is $4^{\circ}$. For the particle with the largest parallax, the cusp of the left particle marked as 6 , the resulting variation of the $\mathrm{z}$-coordinate is about $\pm 0.3 \mu \mathrm{m}$ (the $\mathrm{z}$-coordinate is $2.17 \mu \mathrm{m}$ for $\theta=26^{\circ}, 1.88 \mu \mathrm{m}$ for $\theta=30^{\circ}$, and $1.67 \mu \mathrm{m}$ for $\theta=34^{\circ}$; confirm plot, although slightly tilted: 70 pxl correspond to $\left.1.8 \mu \mathrm{m}\right)$. The largest distance, occurring between structure 2 and 6 , varies by $\pm 0.33 \mu \mathrm{m}$ (the distance is $2.33 \mu \mathrm{m}$ for $\theta=26^{\circ}, 2 \mu \mathrm{m}$ for $\theta=30^{\circ}$, and $1.8 \mu \mathrm{m}$ for $\theta=34^{\circ}$; confirm plot: $77 \mathrm{pxl}$ correspond to $1.97 \mu \mathrm{m})$. Thus, the proof of principle has been shown that stereo experiments using a capillary as sample holder can be done with the CSXM, but the accuracy has to be improved (chapter 7).

\subsubsection{Phaeozem with haematite}

To show that not only in the model system clay-iron-oxide the kind of interactions as described in sections 6.3.1, 6.4.1, and 6.5.1 happen, comparable experiments were performed with phaeozem and haematite. Figure 6.34 shows an X-ray micrograph of an aqueous phaeozem sample where haematite has been added. As extended clusters of soil colloids were expected, the flat holder has been used for this experiment. The left image was taken at $\mathrm{E}=704 \mathrm{eV}$ below the iron $\mathrm{K}$-absorption edge, the right one shows the same region but taken at the absorption maximum at $710 \mathrm{eV}$. Exposure times for both images were 7.2 seconds with $2048 \times 2048$ pixels. The pair of images shows a part of a cluster with a large particle in the image centre, probably a clay particle. The haematite particles are located just at the edge of the large clay platelet, indicating electrostatic interaction as in the case of the kaolinite sample discussed previously (chapter 6.3.1). 

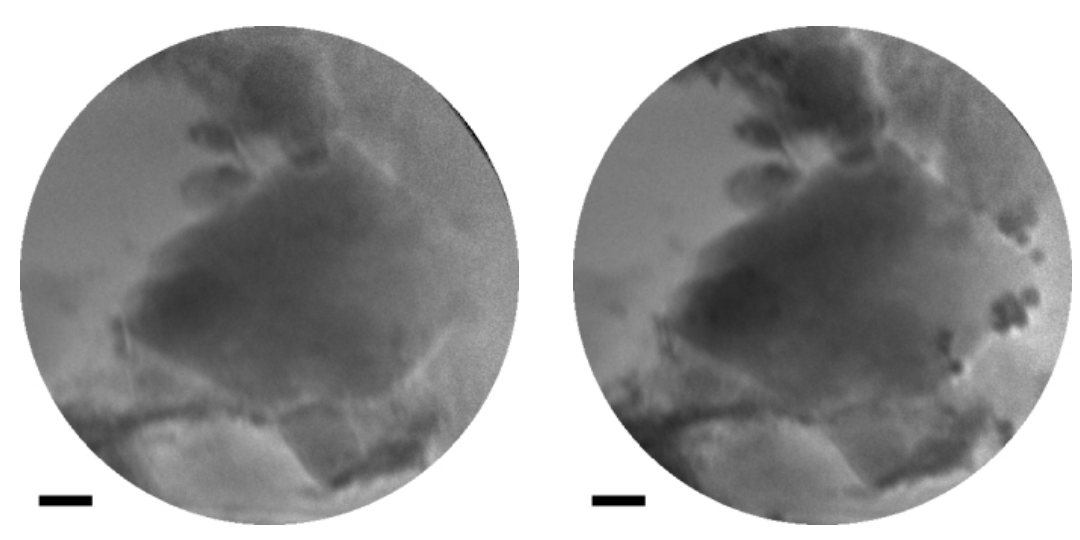

Fig. 6.34: Aqueous calcaric phaeozem sample with haematite colloids between two $\mathrm{Si}_{3} \mathrm{~N}_{4}$ membranes; X-ray micrographs taken at $E=704 \mathrm{eV}$ (left) and $E=710 \mathrm{eV}$ (right). The scale bar in the left corner at the bottom indicates $500 \mathrm{~nm}$. Images were taken with the $X M-1$.

To show the influence on particle conformation of haematite added to an aqueous calcaric phaeozem sample as a progression in time, three pairs of stereo micrographs taken at the STXM are presented in figure 6.35. The tilt angle was $12^{\circ}$ for all three stereo pairs, where the upper images and the lower images were taken under equal tilt positions, respectively. The first pair of stereo images on the left side shows a cluster of a dispersion of pure phaeozem filled into a capillary. A cluster of phaeozem particles of different sizes is shown. The image pair presented in the middle of figure 6.35 shows the same phaeozem cluster, but after the addition of haematite. The lower image is taken $12 \mathrm{~min}$ and the upper image $38 \mathrm{~min}$ after the addition. Structural changes are visible compared to the images taken before the injection of haematite. The image pair shown on the right of figure 6.35 is taken from the same phaeozem cluster after the third addition of dispersed haematite to the capillary, 2 hrs 40 min after first haematite addition. For the upper image, the image recording started $44 \mathrm{~min}$ after the third haematite addition, and $55 \mathrm{~min}$ for the lower image. Further changes in the cluster arrangement are visible.

To determine the changes induced by the addition of haematite, the stereo images shown in figure 6.35 were processed with xstereo. The markers applied to the first stereo pair are shown in left of figure 6.36. Right beside, a view of the revealed plot of the marked structures is presented. xstereo processing of the second and third stereo pair presented in figure 6.35 middle and right are not shown explicitly here. The same structures were marked and the revealed spatial distances are included in the following discussion. The spatial distance between the structures marked as 1 and 2 stays stable 


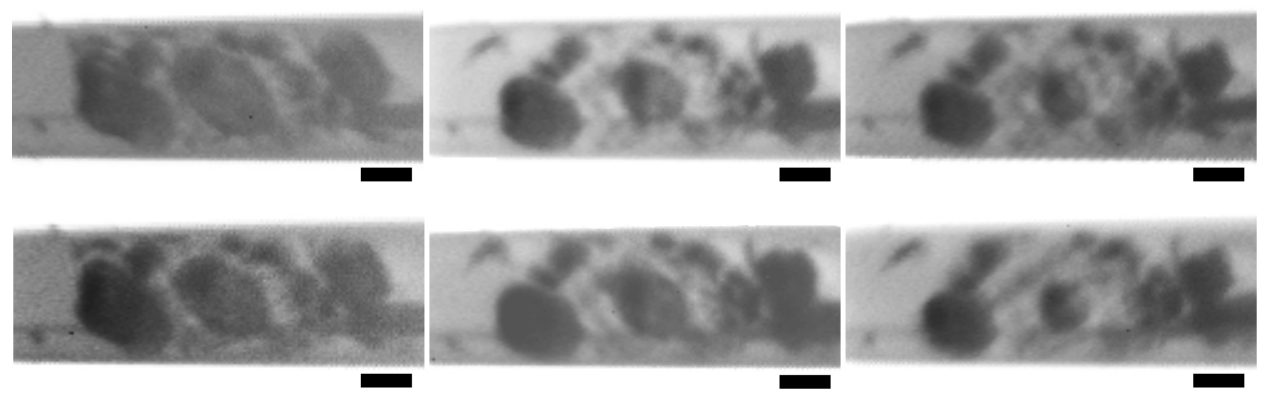

Fig. 6.35: Three pairs of stereo micrographs of aqueous calcaric phaeozem in a capillary. The upper images were taken at equal capillary position, and the lower ones under a tilt angle of $12^{\circ}$. Left: Pure aqueous phaeozem sample. Middle: Same phaeozem cluster after first addition of a haematite dispersion. Imaging time $12 \mathrm{~min}$ (lower image) and $38 \mathrm{~min}$ (upper image) after addition of haematite. Right: Same phaeozem cluster after third addition of haematite dispersion, $2 \mathrm{hrs} 40 \mathrm{~min}$ after first haematite addition. Imaging time $44 \mathrm{~min}$ (upper image) and $55 \mathrm{~min}$ (lower image) after third haematite addition. All images taken with the STXM at $E=400 \mathrm{eV}$ with $50 \mathrm{~nm}$ pxl size and an exposure time of $18 \mathrm{~ms}$ per pixel. The scale bars indicate $1 \mu \mathrm{m}$
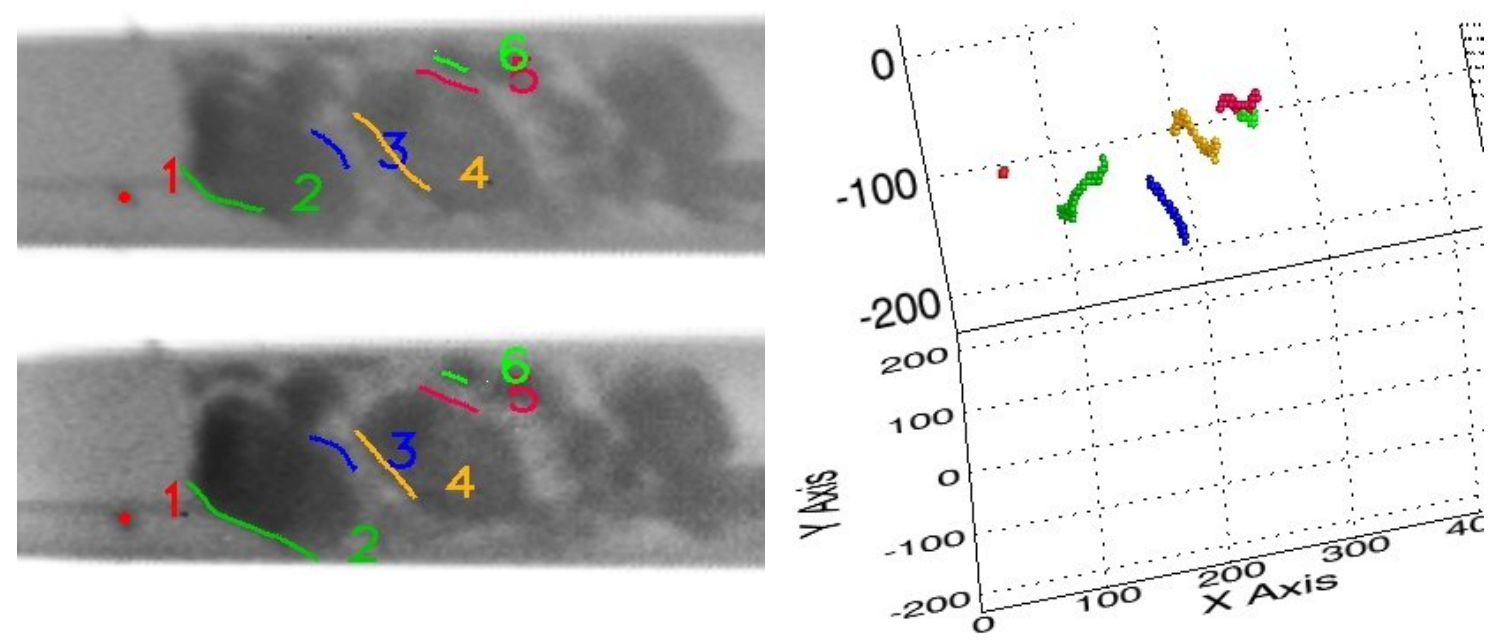

Fig. 6.36: xstereo processing of sample structures of the first pair of micrographs shown on the left in figure 6.35 . 

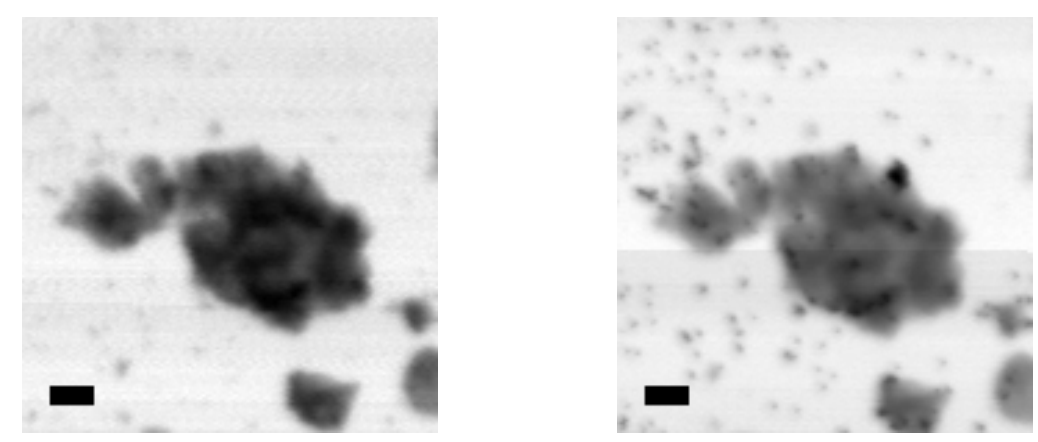

Fig. 6.37: Humic substances extracted from phaeozem mixed with haematite dried onto a $\mathrm{Si}_{3} \mathrm{~N}_{4}$ membrane. For visualisation of the haematite distribution, the micrographs were taken under two different energies: $700 \mathrm{eV}$ (left) and $708 \mathrm{eV}$ (right). The exposure time was $70 \mathrm{~ms}$ per pixel, the image size $18 \times 18 \mu \mathrm{m}^{2}$ on $150 \times 150$ pixel $^{2}$. Images taken with the TwinMic. The scale bars indicate $2 \mu \mathrm{m}$.

at $1.1 \mu \mathrm{m}$ within the estimated error range of $0.1 \mu \mathrm{m}$ due to the image quality. The distance between the particle edges marked as 3 and 4 increases after the addition of haematite from $0.6 \mu \mathrm{m}$ in the first stereo pair (figure $6.36 \mathrm{left}$ ) to $0.8 \mu \mathrm{m}$ in the second stereo pair (not presented xstereo processed, confirm figure 6.35 middle) and $0.9 \mu \mathrm{m}$ in the third stereo pair (not presented xstereo processed, confirm figure 6.35 right). Also the distance between the edges marked as 5 and 6 increases continuously: Initially, the distance is $0.7 \mu \mathrm{m}$, and changes to $0.9 \mu \mathrm{m}$ in the second stereo pair and $1.1 \mu \mathrm{m}$ in the third stereo pair.

However, the increase in distance is due to the shrinkage of the three initially large particles, exceedingly developed in the middle particle. The particles have probably an organic coating, which is radiation sensitive and would explain the shrinkage as a result of radiation damage. As in the case of the experiment with montmorillonite and haematite performed with the STXM (chapter 6.5.1, figure 6.20), it is also neccessary to perfom this experiment at an instrument capable to provide iron mapping, in order to identify haematite particles and conclude on their influence on the detected changes in particle conformation.

\subsubsection{Humic substances extracted from phaeozem with haematite}

Figure 6.37 shows the haematite distribution within a sample of humic substances fractionated from phaeozem mixed with haematite dried onto a $\mathrm{Si}_{3} \mathrm{~N}_{4}$ membrane. For identification of the iron containing haematite particles, the micrographs were taken with the TwinMic under two different energies: $700 \mathrm{eV}$ (figure $6.37 \mathrm{left}$ ) and $708 \mathrm{eV}$ 
(figure 6.37 right). The exposure time is $70 \mathrm{~ms}$ per pixel, the image size $18 \mathrm{x} 18 \mu \mathrm{m}^{2}$ on $150 \times 150$ pixel $^{2}$. The scale bars indicate $2 \mu \mathrm{m}$. In contrast to the mixtures with clay (chapters 6.3.1, 6.4.1, and 6.5.1), the haematite is distributed homogeneously over the $\mathrm{Si}_{3} \mathrm{~N}_{4}$ membrane, probably included in a thin coating of humic substances. The haematite particles are also situated within the large cluster of humic substance. In the projection image, it is not visible how deep the haematite enters the sponge-like structure of the humic substance. This is an object of further stereo X-ray microscopy in combination with elemental mapping.

\subsection{Orthic luvisol}

Another example for a naturally occurring common soil is orthic luvisol (German: Parabraunerde) (chapter 5.4). An X-ray micrograph of pure soil sample looks similar to the calcaric phaeozem sample (figure 6.32) and is not shown here.

\subsubsection{Orthic luvisol with haematite}

Figure 6.38 shows a pair of stereo micrographs of an aqueous sample of orthic luvisol with haematite between two $\mathrm{Si}_{3} \mathrm{~N}_{4}$ membranes. The tilt angle is $14^{\circ}$ around the vertical image axis. The images were taken at $368 \mathrm{eV}$ with the CSXM. The scale bars indicate $2 \mu \mathrm{m}$. An extended cluster of particles with different sizes, shapes and absorption contrasts is visible. The longish structure can be identified as platelet, as its width in the projection images changes drastically by the vertical stereo tilt. Attached to the left side is another platelet whose left edge is surrounded by a cluster of small particles of weak absorption and not clearly visible. Again left of this platelet and apparently connected at the bottom cusps, a slightly smaller particle is situated. Attached or close to its top and left edge, several smaller particles can be seen. On the upper part of the longish platelet, a cluster of small particles, probably haematite according to the shape, extends to the left. On the bottom edge of the longish platelet, a smaller, weakly absorbing particle is attached and another one in small distance left below it. These particles are blurred in the images, while a very fine particle right to them appears well focused at least in the first stereo image (figure 6.38 right). This fuzziness, also visible in right parts of the entire assembly and the right edge of the longish platelet, is either a sign for depth, or for particle motion, and has to be classified.

As performed for previously discussed stereo images taken with the CSXM, each image of figure 6.38 is formed by the addition of single micrographs taken with $60 \mathrm{~s}$ 

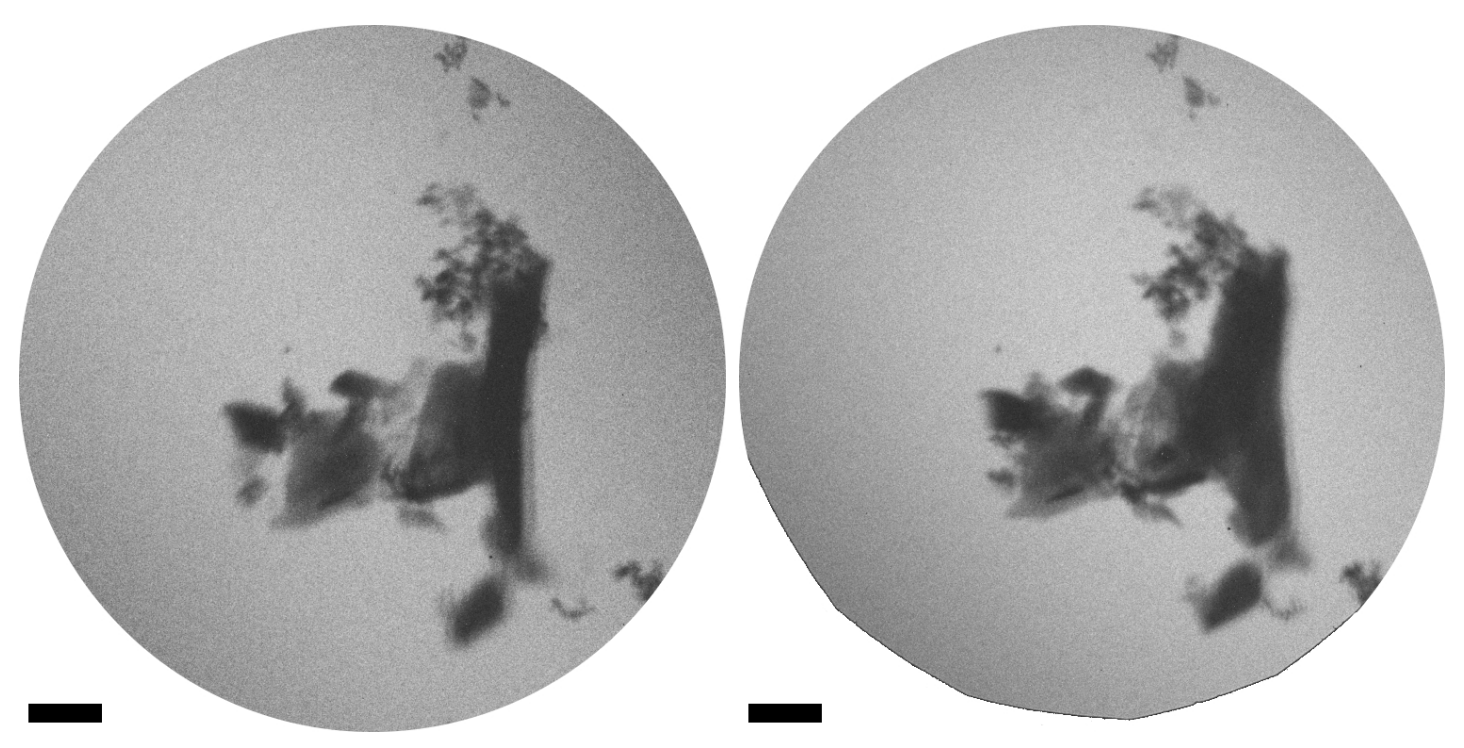

Fig. 6.38: Stereo images of an aqueous sample of orthic luvisol particles with haematite between two $\mathrm{Si}_{3} N_{4}$ membranes. The tilt angle is $\Delta \theta=14^{\circ}$ around the vertical image axis. Each image is formed by adding single micrographs of $60 \mathrm{~s}$ exposure time each. The left image is created by 8 , the right one by 10 single micrographs. Within the added micrographs, slight movements occurred (details in figure 6.39), resulting in a minor blurring. Images taken with the CSXM at $E=368 \mathrm{eV}$, the scale bars indicate $2 \mu \mathrm{m}$.

exposure time, respectively, to detect sample movements during the stereo experiment. For the first stereo image, a series of 8 micrographs was recorded, and for the second stereo image a series of 10 micrographs. The first and the last micrograph of each image series is presented in figure 6.39 left and middle. The images shown on the left side correspond to the left micrograph in figure 6.38, the middle images to the right micrograph in figure 6.38, accordingly. The left image series was taken within $12 \mathrm{~min}$ before tilting the sample holder. 13 min afterwards, the second series of stereo images (figure 6.39 middle) was recorded within 11 min, resulting in a total time span of 36 min. Both top images show the first micrographs and the bottom images the last (8th and 10th, respectively) micrographs of the corresponding image series. For a final check for possible sample movements during the entire time span of the experiment, a control micrograph was recorded subsequently to both series of stereo images at the initial tilt position of the sample and is shown in figure 6.22 top right. The time lag between recording this micrograph and the first one of the stereo experiment (figure 6.39 top left) is $52 \mathrm{~min}$. 

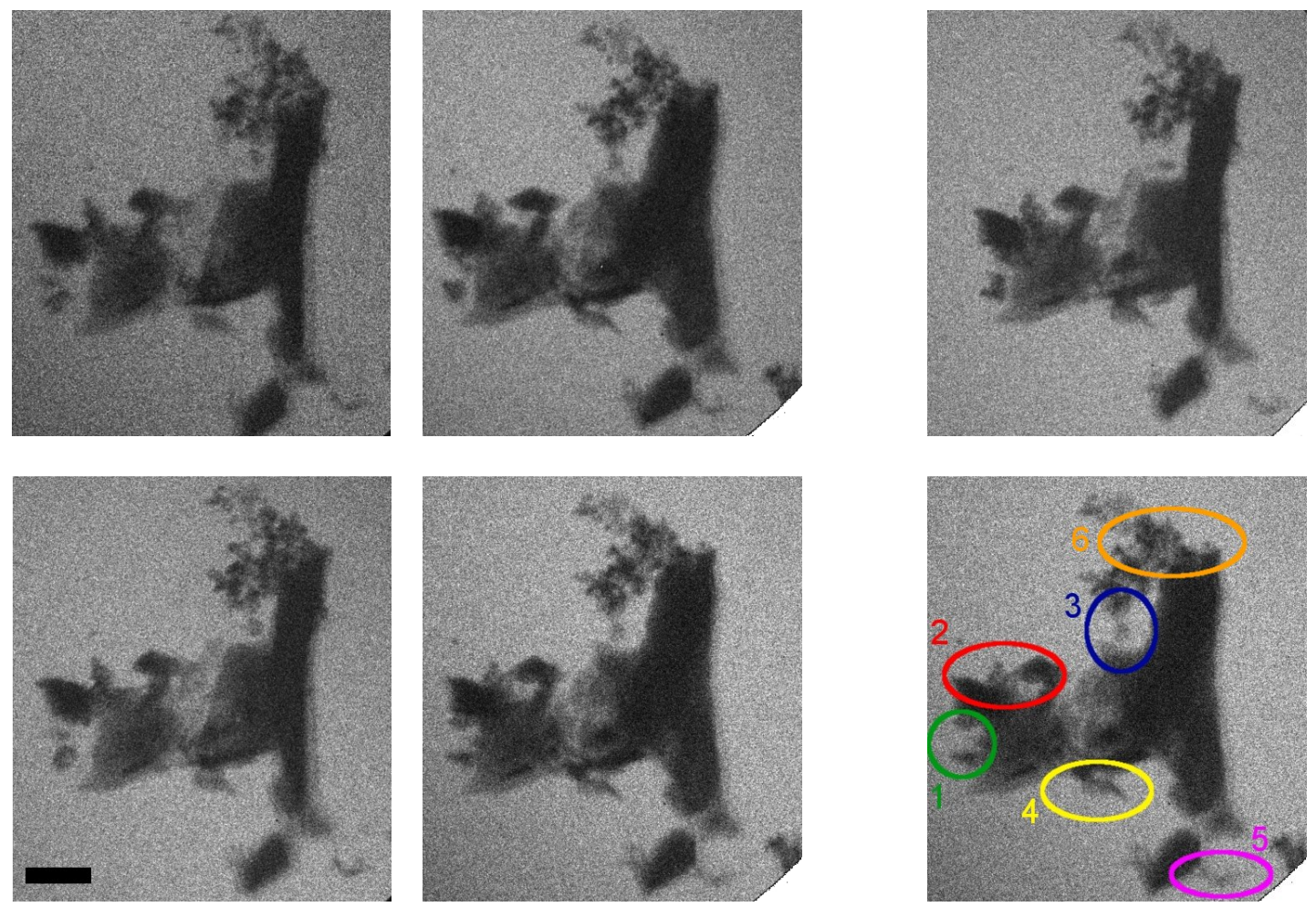

Fig. 6.39: Single micrographs of the previous sample to identify particle movement during the acquisition of the stereo image series shown in figure 6.38. Left and middle: Sequences of the added stereo images of aqueous sample of orthic luvisol with haematite shown in figure 6.38 with $60 \mathrm{~s}$ exposure time. Images on the left side correspond to the left image in figure 6.38 , middle images accordingly to right image in figure 6.38. The left image series was taken before tilting the sample for recording series of images shown right. The top images show first micrographs, the bottom images the last micrographs (8th and 10th) of image series. Top right: Micrograph taken after recording both series of stereo images at the initial tilt position, corresponding to the micrographs of the first image series (left). Bottom right: Regions of interest marked for detailed discussion (chapter 6.7). The micrograph is identical to the one shown top right. The exposure time was $60 \mathrm{~s}$ per micrograph. Images were taken with the CSXM at $E=368 \mathrm{eV}$, the scale bar indicates $2 \mu \mathrm{m}$. 
The regions of interest for the determination of distances, selected by comparing the stereo images shown in figure 6.38, are marked in the micrograph shown in figure 6.39 bottom right. In region number 1, the spatial distance of the small particle to the platelet edge is of interest. However, the single micrographs of the tilt series reveal a continuous approximation of the particle during the experiment, resulting in a total drift of about $17 \mathrm{~nm}$ per minute. Due to this enduring motion, stereo analysis is not possible. For region number 2, the same effect is detected. The middle and left particle turn up towards the right particle. Therefore, spatial distances between these particles cannot be determined. The small particle in region number 3, probably a haematite particle, is situated between the haematite cluster and the middle soil platelet. Hence, the interaction to study is faster than the stereo experiment: Within the image series, the small particle drifts slowly, with a velocity of about $14 \mathrm{~nm}$ per minute towards the platelet, and has reached a position nearly attached to it in the control micrograph. A determination of its spatial position is not possible. Also the small platelet in region number 4 keeps on converging the edge of the larger platelet above it. The position of the fine structure in region number 5 is fix, but the platelets left to it moves upwards. Thus, a distance determination is not possible. Also the longish platelet, situated above the small particle of region number 5, moves slightly upwards, which causes the blur of its right edge, as well as changes of its position relative to the haematite cluster selected as region number 6 . Thus, only the distance between the edge of the large platelet in the middle of the assembly and the small particle in region number 2 bottom right can be determined by xstereo processing to be $1.5 \mu \mathrm{m}$. The platelet and the haematite conglomeration lie with variations of $300 \mathrm{~nm}$ within the same z-plane, while the small particle of region number 5 has a distance in z-direction of $1.5 \mu \mathrm{m}$ to them. The maximal distance in z-direction detected in the xstereo processing exists between the mentioned structures of region 2 and 5 and adds up to $2.8 \mu \mathrm{m}$ (figures of corresponding xstereo processing are not presented).

Supporting the conclusions made for the experiment of montmorillonite with nanotubes (chapter 6.5.2, figure 6.22), the stereo investigation of this sample requires shorter exposure times. This would even enable to take several pairs of stereo images and thus to examine the particle motion, which has been shown to mainly involve velocities beyond $20 \mathrm{~nm}$ per minute. Therefore, the particles move slow enough so the change in position is beyond the resolution limit for X-ray micrographs of typical exposure times in the range of seconds (chapter 7 ). The combination with elemental mapping has to be 

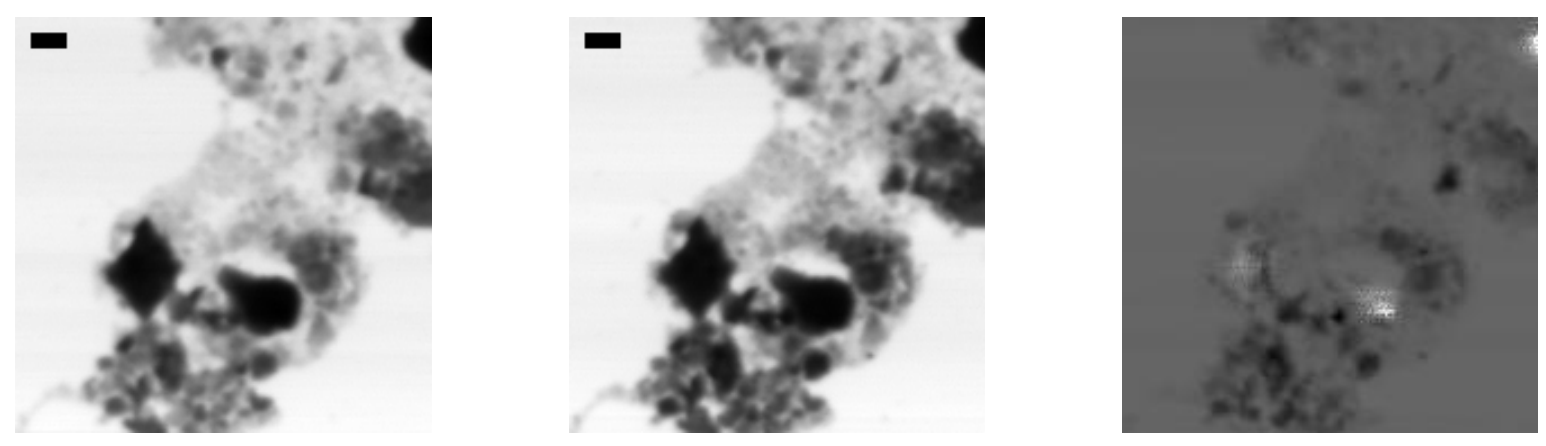

Fig. 6.40: Iron detection in a dystric planosol sample. Left and middle: Micrographs of a sample of dystric planosol dried onto a $\mathrm{Si}_{3} \mathrm{~N}_{4}$ membrane. The images were taken at $700 \mathrm{eV}$ (left) and $708 \mathrm{eV}$ (middle) with an exposure time of $75 \mathrm{~ms}$ per pixel, the image size is $25 \times 25 \mu \mathrm{m}^{2}$ on $200 \times 200$ pixel $^{2}$. The images were taken with the TwinMic. The scale bar indicates $2 \mu \mathrm{m}$. Right: Iron distribution map created as the logarithm of the division of the images besides. Content of iron is visible as darker areas.

performed at other X-ray microscopy stations to clearly distinguish between haematite and luvisol particles.

\subsection{Dystric planosol}

As third example of a naturally occurring soil, dystric planosol (German: Pseudogley) had been chosen (chapter 5.4. Figure 6.40) left and middle shows two micrographs of dystric planosol dried onto a $\mathrm{Si}_{3} \mathrm{~N}_{4}$ membrane. The images were taken with the TwinMic at $700 \mathrm{eV}$ (figure $6.40 \mathrm{left}$ ) and $708 \mathrm{eV}$ (figure 6.40 middle). The exposure time was $75 \mathrm{~ms}$ per pixel, the image size is $25 \times 25 \mu \mathrm{m}^{2}$ on $200 \times 200$ pixel $^{2}$. The scale bars indicate $2 \mu \mathrm{m}$. Two extended, strongly absorbing particles are situated in the middle of the image field, within a dense layer of smaller particles. Some particles or rather sample areas show an increase in absorption in the micrograph taken above the iron absorption edge (figure 6.40 middle). In contrast to particles of very high iron content, the iron content of the planosol particles is less and the respective particles show also an absorption of X-rays below the iron absorption edge. Therefore, the distribution of these iron containing regions is visualised in the distribution map created as the logarithm of the division of the images besides (chapter 2.4.2) shown in figure 6.40 right. Iron appears as dark areas in the regions in the bottom of the image field, between the two large, strongly absorbing particles, above the right large particle and in the top right corner of the image field. These regions show also a higher absorption relative to the surrounding 


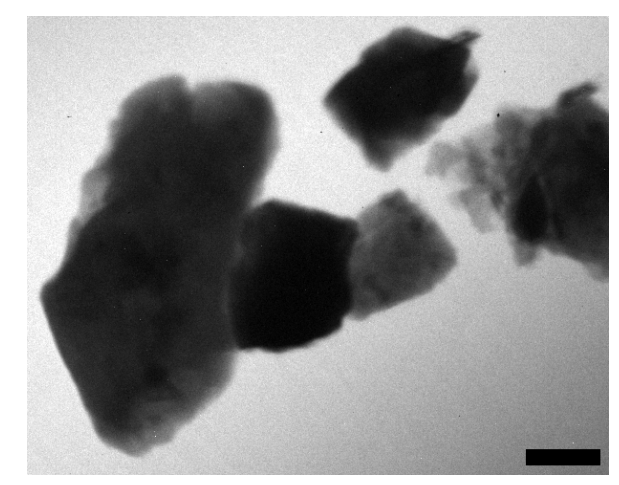

Fig. 6.41: Micrograph of an aqueous sample of dystric planosol particles. The image were taken with the CSXM at $E=368 \mathrm{eV}$ as sum of 9 micrographs with $60 \mathrm{~s}$ exposure time each. The scale bar indicates $2 \mu \mathrm{m}$.

areas in the micrograph taken below the iron absorption edge. This gives rise to the assumption that they are regions of larger thickness and therefore with a higher content of carbon. However, three smaller regions show a notable increase in absorption when crossing the iron absorption energy, one in the upper part of a larger particle in the middle of the bottom assembly of small particles, one attached to the bottom edge of the right large particle, and one at the border of the region of high absorption in the top right image field. Here, an agglomeration of iron is clearly visible.

Figure 6.41 presents a micrograph of aqueous dystric planosol particles taken with the CSXM at $\mathrm{E}=368 \mathrm{eV}$. The image is created as a sum of 9 single micrographs taken subsequently with 60 s exposure time each, and particle motion can be excluded here as discussed previously for experiments perfomed with the CSXM. An assembly of five large particles is visible, where three of them are attached to each other and two are in short distance. The single particle at the right edge of the image field is surrounded by a cluster of smaller particles. The existence of small particles in the pure planosol sample, also detected in the dried state as seen in figure 6.40 left and middle, makes it difficult to identify added small particles as carbon nanotubes or haematite just by appearance and without elemental mapping. On the other hand, due to the natural iron content of luvisol, mapping results should to be combined with particle sizes. Thus, small, spherical haematite particles with very weak absorption in the micrograph taken below the absorption edge can be distinguished from iron containing luvisol particles, as discussed in the following. 


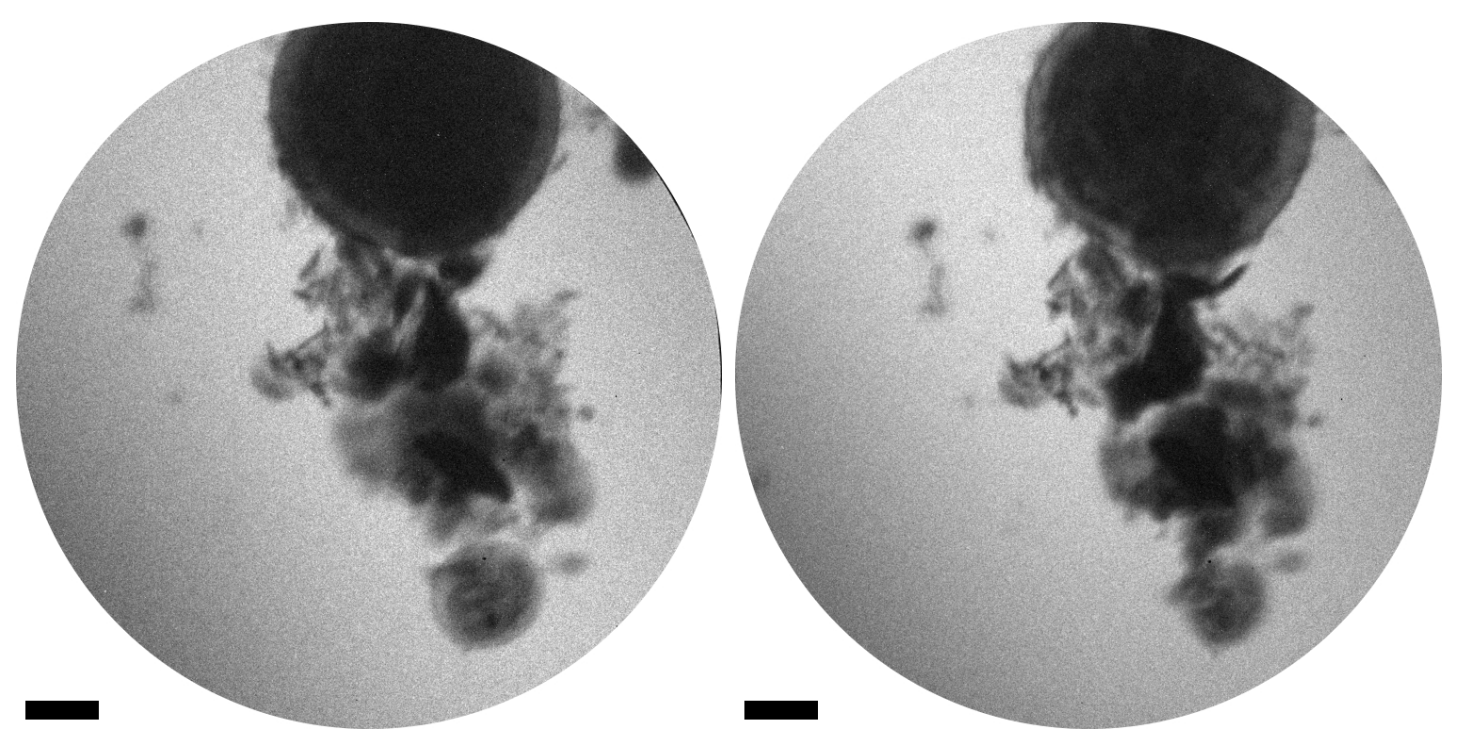

Fig. 6.42: Stereo micrographs of an aqueous sample of planosol particles with haematite. The tilt angle is $\Delta \theta=14^{\circ}$ around the vertical image axis. Each image is a sum of 10 micrographs taken with $60 \mathrm{~s}$ exposure time, respectively. The images were taken with the $C S X M$ at $E=368 \mathrm{eV}$, the scale bars indicate $2 \mu \mathrm{m}$.

\subsubsection{Dystric planosol with haematite}

The mixture of dystric planosol with haematite has been examined in aqueous state between two $\mathrm{Si}_{3} \mathrm{~N}_{4}$ membranes with the CSXM. Figure 6.42 shows a pair of stereo micrographs taken under a vertical tilt angle of $14^{\circ}$ at $368 \mathrm{eV}$. The scale bars indicate $2 \mu \mathrm{m}$. For each image, 10 single micrographs were taken subsequently with an exposure time of $60 \mathrm{~s}$, respectively, to determine sample motion during the stereo experiment as performed previously (chapters 6.5.2, 6.6, and 6.7.1). The first image series was recorded within $13 \mathrm{~min}$. After a time lag of $11 \mathrm{~min}$ due to the manual tilt of the sample and realignment, the second image series was also recorded within $13 \mathrm{~min}$.

A large, spherical particle is visible in the top region of the image field, to whose bottom edge an assembly of various particles is attached. This assembly consists in three larger particles of strong absorption contrast, surrounded by clusters of small, most of them weakly absorbing particles. Very closely situated, probably attached to the bottom cusp of this assembly is another spherical particle. This particle appears blurred in second stereo image (figure 6.42 right), as well as the bottom region of the particle assembly. Therefore, the single micrographs of the image series are considered in the following. 

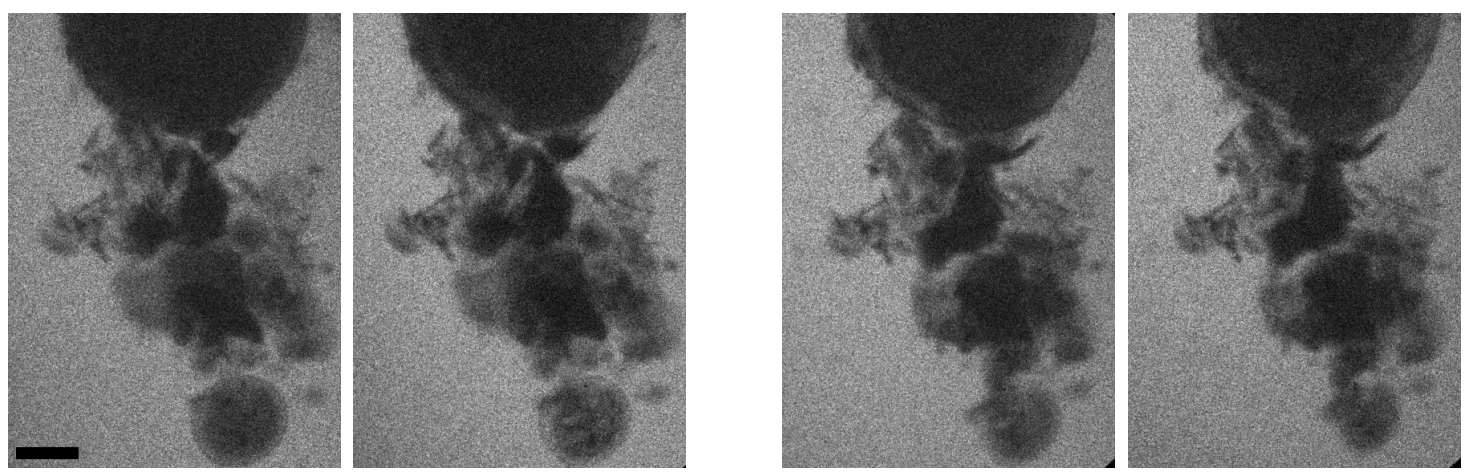

Fig. 6.43: First and 10th sequence images of $60 \mathrm{~s}$ exposure time of the added stereo images shown in figure 6.42. The left pair of micrographs corresponds to first stereo image (figure 6.42 left), where the first image of the sequence is the left one, and the 10th is the right one. The right image pair shows analoguesly sequence images of the second stereo image (figure 6.42 right), recorded after the left one. Slight shrinking of some structures is visible. The scale bar indicates $2 \mu \mathrm{m}$.

Each first and 10th sequence image of $60 \mathrm{~s}$ exposure time of the added stereo images (figure 6.42) are presented in figure 6.43. The scale bar indicates $2 \mu \mathrm{m}$. The left pair of micrographs corresponds to the first image series (figure 6.42 left), where the first image of the sequence is the left one, and the 10th is the right one. The image pair at the right side corresponds analoguesly to the second image series (figure 6.42 right), recorded in chronological order and time periods as indicated above.

In the course of the image series, a slight shrinkage of some structures can be detected. Especially the spherical structures in the top and bottom region of the image field show this effect strongly. This shrinkage caused the fuzziness in of the spherical structures in the stereo images (figure 6.42) and impedes xstereo processing. However, concerning the motionless structures, the maximal distance in z-direction for the assembly is found to be $2.9 \mu \mathrm{m}$, occurring between the bottom cusp of the strongly absorbing particle in the lower part of the assembly of smaller, weakly absorbing particles, and several small particles in the outer left part of the assembly. Further details and figures of the corresponding xstereo processing are not presented.

Similar to the shrinkage of particles detected within the image series of calcaric phaeozem with haematite (chapter 6.6.1, figure 6.35), the assumed reason for it in this sample of planosol with haematite is the content of radiation sensitive organic matter. Probably, larger inorganic soil particles are coated with an organic layer diminishing with increasing X-radiation dosage. For the big spherical particle, an outer layer of 
less absorbing matter of about $500 \mathrm{~nm}$ thickness can be seen in both projection images. The small spherical particle shows a similar layer, although not so homogeneously distributed, as the layer thickness in the bottom part is just about $180 \mathrm{~nm}$. Stereo microscopy provides the advantage of requiring just two micrographs, thus, in principle the spatial distribution of radiation sensitive samples is available by this method. However, to image these radiation sensitive samples, the dosage has to be minimised. The possibilities for this purpose are discussed in chapter 7. Again, an identification of haematite particles is not possible just by the particle shape. A combination of elemental contrast has to be performed at suitable X-ray microscopy stations, e.g. the XM-1.

\subsection{Influence of $\mathrm{pH}$}

Three systems containing additional haematite have been studied in elemental contrast, kaolinite, montmorillonite, and the soil phaeozem. It is known that clay minerals show a negative surface charge in combination with positively charged edges at low $\mathrm{pH}$ values (chapter 5.1). When increasing this value, the edge charges change to negative as well. For kaolinite, the point of zero charge, i.e. the transition from positive to negative edge charges, lies around $\mathrm{pH} \approx 2.7-4.1$ (chapter 5.3.2, table 5.3.2). The haematite dispersion used here was measured to $\mathrm{pH} \approx 3-4$. The point of zero charge for haematite is around $\mathrm{pH} \approx 8.5$, resulting in positively charged particles added to the kaolinite dispersion. As the kaolinite has been dispersed with deionised water at neutral $\mathrm{pH}$, and only little quantities of haematite have been added in order to not change the $\mathrm{pH}$ drastically, the $\mathrm{pH}$ of the mixture is approximately 5 . Thus, the edge charge of the kaolinite is negative, resulting in an electrostatic attraction of the haematite particles to these edges. This can be seen in figure 6.8 .

The phaeozem soil has a measured $\mathrm{pH}$ of 8.4, with just negligible changes when small amounts of haematite are added. As a $1 \%$ soil dispersion in deionised water has been used for the experiments, a $\mathrm{pH}$ value just slightly below 8 can be assumed. Here, the edge charge of the clay platelets is negative, however the haematite particles are still slightly positively charged. Again, an electrostatic attraction of these edges towards the haematite particles can be expected. The spatial arrangement of haematite particles around the edges of the soil particles visible in figure 6.34 supports this expectation.

The montmorillonite dispersion has a $\mathrm{pH}$ of approximately 6 , resulting in a $\mathrm{pH}$ of the mixture measured to $\mathrm{pH} \approx 5$. Haematite is positively charged at this $\mathrm{pH}$ value, however, for montmorillonite, the point of zero charge given in literature varies widely between $\mathrm{pH}$ values of about 4 to 6 (chapter 5.3.2, table 5.3.2), dependent among others on its origin. 
In the right image of figure 6.15 it becomes clear that the haematite particles arrange themselves at the edges of the montmorillonite cluster. Therefore, it can be assumed that the edge charge of the montmorillonite particles is slightly negative, leading to a point of zero charge below 5. When looking at the subcluster depicted in figure 6.18 and using xstereo to derive the distances and the plot shown in figure 6.19, it becomes clear that two of the haematite particles seen there are very close to the clay platelets, and three particles are attached to one of the support membranes. As the resulting $\mathrm{pH}$ value is close to the point of zero charge, the electrostatic forces are very weak. This has been sufficient to attract all haematite particles to the clay clusters during sample preparation in a test tube. However, in the vicinity of the support membranes, probably charged as well, the weak interaction is not strong enough to prevent the haematite particles from switching places.

Furthermore, the direct contact due to electrostatic attraction between clay and haematite could be detected in a mixed dispersion of nontronite, also with a measured $\mathrm{pH}$ value around 6 between the points of zero charge of nontronite and haematite.

The results from stereo microscopic analysis presented here can be understood as a starting point for a thorough investigation of the electrostatic interaction within these systems. The possibilities and requirements for further experiments are discussed in chapter 7 . 


\section{Chapter 7}

\section{Conclusion and Outlook}

The aim of this work was the application of stereo X-ray microscopy to aqueous samples from the environment and the extension to the investigation of dynamical behaviour of the samples. The results can be divided into three parts, an instrumental, a methodical, and a soil scientific one.

On the instrumental part, the performance of stereo experiments at various X-ray microscopy stations was described. Mechanical adaptions for stereo experiments were done at the XM-1, where the detachable tilt stage was implemented (chapter 4.1.1). At the STXM, the implementation of the detachable tilt stage required more modifications (chapter 4.2.1). An aluminium adapter was designed to mount the tilt stage to the microscope maintaining enough distance not to hit the vacuum window or the vacuum window mount with the capillary. Due to the small distance between the zone plate vacuum vessel and the detector vacuum vessel, the tilting is performed via a two-parts axle, which had to be inserted into the object plate of the STXM. Tilting is executed by a stepper motor. Another important enhancement is the equipment of the visible light microscope of the STXM with a USB digital camera for higher accuracy in prealignment and less focusing effort with X-radiation, resulting in a significantly lower X-ray dose. The stereo tilting stage of the CSXM (chapter 4.1.2) was designed and built by the KTH group according to the requirements elaborated in joint discussions.

On the methodical part, the self-written programme xstereo was enhanced and a graphical user interface was created for a user friendly and flexible handling. The requirements for the alignment of two stereo X-ray micrographs were discussed and an appropriate alignment routine was implemented to xstereo (chapter 3.7).

It has been verified that taking several X-ray micrographs is possible under ambient conditions for soil samples with low organic content. Thus, no cryo fixation is necessary 
to prevent detectable radiation damage and the investigation of dynamics is in principle possible. However, the amount of X-ray images necessary for a stereo image pair has to be reduced to a minimum by good prealignment of the sample, accurate focus access, and exact tilting.

Two types of sample holders, capillaries and fluidic cells based on coated $\mathrm{Si}_{3} \mathrm{~N}_{4}$ membranes (chapter 3.1) were tested for possible sample manipulation during the stereo experiment. Samples within capillary tips could be manipulated in-situ by injection of additional dispersions. When using capillary tips of diameters smaller than $3 \mu \mathrm{m}$, like mandatory when imaging aqueous samples at the iron L-absorption edge, it is difficult to fill them with sample particles of reasonable sizes. Furthermore, only samples providing a high absorption contrast for small particles are suitable. However, when using energies below the K-absorption edge of oxygen, thus within the water-window, capillary diameters of up to $10 \mu \mathrm{m}$ can be used, which are accessible for larger particles. A possible drawback is the obstruction of the tapering region of the capillary by large, dense clusters. Single particles small enough to access the capillary tip dispose of a high mobility, which may complicate sharp imaging, but means an undisturbed formation of the particle clusters. On the contrary, a stack of two $\mathrm{Si}_{3} \mathrm{~N}_{4}$ membranes exerts a force perpendicular to the membranes due to attraction caused by the capillary forces. Therefore, larger platelet-like particles are often orientated parallel to the membranes. The fluidic cell sample holder could not reach the expectations in X-ray experiments, as the evoked aggregations were too small to be stuck between the foils, or the water layer often was too thick as water crossed the coating. Here, further developments have to be done. The standard sample holder for aqueous specimen, a set of two uncoated $\mathrm{Si}_{3} \mathrm{~N}_{4}$ membranes, enabled to image aqueous samples showing dynamical behaviour probably due to diffusion. These drifts with velocities measured to be in the range of 10 to $20 \mathrm{~nm}$ per minute (chapter 6 ) are slow enough to be investigated by stereo soft X-ray microscopy, when each set of stereo micrographs is taken within one minute.

With regard to the soil science, experiments were initially performed with model systems of clay and iron oxides. Subsequently, more complex, clay containing soil samples were investigated similarly. The morphology of those samples was described in both dried and aqueous states. The interaction of mixed systems of clay and soil particles with additive haematite was investigated, both in an aqueous state and subsequently dried. The haematite particles within the composed samples were identified either due to assumptions with regard to the particle shape, or by elemental mapping at the Fe L 3 absorption edge. The spatial arrangement of these mixed samples was revealed by 
xstereo processing, as the distances between clay edges and haematite particles enable conclusions to the charges, which correlate to the $\mathrm{pH}$ value of the particular systems. It could be demonstrated that at $\mathrm{pH}$ values where clay edges are positively charged and haematite particles are negatively charged, attachment of haemtatite particles to the clay edges are detectable with the presented technique. Thus, the determination of the point of zero charge of the investigated clays and soils could be verified or even specified. A first step towards the characterisation of the haematite distribution within humic substances was made.

As another investigation area for particle interaction, carbon nanotubes were described and added to clay and soil samples. The carbon nanotube distribution within mixed sample systems was detected in dried samples by elemental mapping around the carbon K absorption edge.

Changes within the arrangement of aqueous samples due to drifts were detected. The in-situ addition of haematite particles or carbon nanotubes evoked strong morphological changes. Possible reasons can be either a change in $\mathrm{pH}$ due to the addition of the haematite dispersion and the thereby changed surface charges of the clay particles, or the influence of carbon nanotubes on the aggregation properties.

The investigations of the interactions of colloidal soil particles will be continued with further, precise correlations to $\mathrm{pH}$ values and concentrations. Also the spatial distribution of haematite particles within humic substances will be investigated revealing the penetration depth of the haematite particles into the highly porous clusters of humic substances. A further possible field of investigation is the electrophoresis of soil colloids, which leads to the determination of the isoelectric point. For this, an electric field has to be established at the sample region in the microscope.

As shown by the first results presented here, stereo soft X-ray microscopy is a technique very well suited for this purpose. Elemental contrast is necessary for the secure identification of added iron oxide particles or carbon nanotubes. To combine the stereo investigation of dynamics, either induced or due to drifts, with elemental contrast, further adaptions of the microscopes providing elemental contrast have to be done. This means for the XM-1 the implementation of an automated tilt device like a stepper motor to the XM-1. At the STXM, the possibility of focusing a capillary tip at energies below the carbon K-absorption edge has to be realised either by a cone mount for the vacuum window or an enlarged driveway of the zone plate.

General requirements for stereo investigations of dynamical processes are exposure times in the range of a second and an automated stereo tilting without time consuming 
re-adjustment. This will be fulfilled also by the CSXM after the planned upgrade with a new high-power laser, decreasing the exposure time for imaging aqueous specimen below $10 \mathrm{~s}$ (Hertz et al., 2009 (29)).

The interaction of soil colloids is still not fully understood. The DLVO theory is being expanded (Grasso et al., 2002 (24)), and models for the aggregation and adsorption processes are an aim of actual investigations (Vormoor, 2002 (90), Kraepiel et al., 1999 (42)). The demonstrated capacity of stereo soft X-ray microscopy in revealing information about spatial arrangements of aqueous colloidal particles at the nanometre scale in combination with (induced) dynamical behaviour and with unambiguous particle identification or detection by elemental contrast enables an new sight onto these interactions. 


\section{Bibliography}

[1] C. Ahl, K. W. Becker, R. G. Jörgensen, and H. Wildhagen. Aspekte und Grundlagen der Bodenkunde. Göttingen und Witzenhausen: Eigenverlag, Germany, 33 edition, 2007.

[2] C. Ahl, H.-G. Frede, S. Gäth, and B. Meyer. Böden aus Löß des Leinetalgrabens und seiner Hochflächen-Umrandung. Mitteilungen der Deutschen Bodenkundlichen Gesellschaft, 42, 359-434, 1985.

[3] S. Aoki, Y. Kagoshima, and Y. Suzuki, editors. X-Ray Microscopy, Proceedings of the 8th Intl. Conf., volume 7 of Conference Series. Institute of Pure and Applied Physics, Tokyo, 2006.

[4] C. Appel, L. Q. Ma, R. D. Rhue, and E. Kennelley. Point of zero charge determination in soils and minerals via traditional methods and detection of electroacustic mobility. Geoderma, 113, 77-93, 2003.

[5] D. Attwood. Soft X-rays and extreme ultraviolet radiation. Cambridge University Press, Cambridge, 1999.

[6] F. Bergaya, B. K. G. Theng, and G. Lagaly, editors. Handbook of Clay Science. Elsevier, 2006.

[7] M. Berglund, L. Rymell, M. Peuker, T. Wilhein, and H. M. Hertz. Compact water-window X-ray transmission microscopy. Journal of Microscopy, 197, 268-273, 2000.

[8] M. Bertilson, O. von Hofsten, J. Thieme, M. Lindblom, A. Holmberg, P. Takman, U. Vogt, and H. Hertz. First application experiments with the Stockholm compact soft X-ray microscope. In: 9th International Conference on X-Ray Microscopy, volume 186 of Journal of Physics: Conference Series (IOP), 012025. 2009. 
[9] M. Born and E. Wolf. Principles of Optics - Electromagnetic Theory of Propagation, Interference and Diffraction of Light. Pergamon Press, sixth edition.

[10] G. Brezesinski and H.-J. Mögel. Grenzflächen und Kolloide. Spektrum Akademischer Verlag, 1993.

[11] C. J. Buckley, N. Khaleque, S. J. Bellamy, M. Robins, and X. Zhang. Mapping the Organic and Inorganic Components of Bone. In: J. Thieme, G. Schmahl, D. Rudolph, and E. Umbach, editors, X-Ray Microscopy and Spectromicroscopy, II-47 - II-55. Springer-Verlag, Heidelberg, 1998.

[12] W. Chao, B. Harteneck, J. Liddle, E. Anderson, and D. Attwood. Soft Xray microscopy at a spatial resolution better than $15 \mathrm{~nm}$. Nature, 435, 1210-1213, 2005.

[13] J. D. Coates, R. Chakraborty, S. M. O'Connor, C. Schmidt, and J. Thieme. The Geochemical Effects of Microbial Humic Substances Reduction. acta hydrochimica et hydrobiologica, 28, 420-427, 2000.

[14] R. M. Cornell and U. Schwertmann. The Iron Oxides: Structure, Properties, Reactions, Occurrences, and Uses. second edition, 2003.

[15] J. R. Dean. Bioavailability, bioaccessibility and mobility of environmental contaminants. Analytical Techniques in the Sciences. John Wiley \& Sons Ltd., West Sussex, England, 2007.

[16] S. Duiker, F. Rhoton, J. Torrent, N. Smeck, and R. Lal. Iron (hydr)oxide crystallinity effects on soil aggregation. Soil Science Society of America Journal, 67, 606-611, 2003.

[17] P. S. F. Scheffer, H.-P. Blume, G. Brümmer, K.-H. Hartge, and U. Schwertmann. Lehrbuch der Bodenkunde. Ferdinand Enke Verlag Stuttgart, Germany, 13rd edition, 1992.

[18] P. Fischer, D. H. Kim, W. Chao, J. A. Liddle, E. H. Anderson, and D. Attwood. Soft X-ray microscopy of nanomagnetism. Materials Today, 9(12), 26, 2006.

[19] L. Garvie and P. Buseck. Ratios of ferrous to ferric iron from nanometre-sized areas in minerals. Nature, 396, 667-670, 1998. 
[20] S.-C. Gleber, C. Knöchel, J. Thieme, D. Rudolph, and G. Schmahl. 3$D$ computer reconstruction of X-ray microscopy objects from stereo images. In: J. Susini, D. Joyeux, and F. Polack, editors, X-Ray Microscopy 2002, volume 104 of Journal de Physique IV Proceedings, 639. EDP Sciences, Les Ulis, 2003.

[21] S.-C. Gleber, J. Sedlmair, M. Bertilson, O. von Hofsten, S. Heim, P. Guttmann, H. M. Hertz, P. Fischer, and J. Thieme. X-ray stereo microscopy for investigation of dynamics in soils. In: 9th International Conference on X-Ray Microscopy, volume 186 of Journal of Physics: Conference Series (IOP), 012104. 2009.

[22] S.-C. Gleber, J. Thieme, W. Chao, and P. Fischer. Stereo soft X-ray microscopy and elemental mapping of hematite and clay suspensions. Journal of Microscopy, 235(2), 199-208, 2009.

[23] S.-C. Gleber, J. Thieme, P. Guttmann, S. Heim, and G. Mitrea. X-ray stereo microscopy at BESSYII to study dynamics. BESSY Annual Report 2006, 234-236, 2007.

[24] D. Grasso, K. Subramaniam, M. Butkus, K. Strevett, and J. Bergendahl. A review of non-DLVO interactions in environmental colloidal systems. Re/Views in Environmental Science \& Bio/Technology, 1, 17-38, 2002.

[25] P. Guttmann, S. Heim, S. Rehbein, B. Niemann, R. Follath, and G. Schneider. X-ray microscopy at the new U41-FSGM beam line. BESSY Annual Report 2007, 297-300, 2008.

[26] S. Heim, P. Guttmann, S. Rehbein, B. Niemann, and G. Schneider. X-ray microscope with mono-capillary condenser. BESSY Annual Report 2007, 510-513, 2008.

[27] B. Henke, E. Gullikson, and J. Davis. X-ray interactions: Photoabsorption, scattering, transmission, and reflection at $E=50-30000 \mathrm{eV}, Z=1-92$. Atomic Data and Nuclear Data Tables, 54, 181-342, 1993.

[28] H. M. Hertz, M. Berglund, G. A. Johansson, M. Peuker, T. Wilhein, and H. Brismar. Compact water-window X-ray microscopoy with a droplet laserplasma source. In: W. Meyer-Ilse, T. Warwick, and D. Attwood, editors, X-Ray Microsocpy, Proceedings 6th Intl. Conf. X-Ray Microscopy, Proceedings of XRM 2000, 721-725. American Institute of Physics, Melville, 2000. 
[29] H. M. Hertz, M. Bertilson, J. Ewald, S.-C. Gleber, O. Hemberg, M. Henriksson, O. von Hofsten, A. Holmberg, M. Lindblom, E. Mudry, M. Otendal, J. Reinspach, P. Skoglund, P. Takman, J. Thieme, J. Sedlmair, R. Tjörnhammar, T. Tuoshimaa, M. Vita, and U. Vogt. Laboratory x-ray micro imaging: Sources, optics, systems and applications. In: 9th International Conference on X-Ray Microscopy, volume 186 of Journal of Physics: Conference Series (IOP), 012027. 2009.

[30] T. Hofmann, T. Baumann, T. Bundschuh, F. von der Kammer, A. Leis, D. Schmitt, T. Schäfer, J. Thieme, K. U. Totsche, and H. Zänker. Aquatische Kolloide I: Eine Übersichtsarbeit zur Definition, zu Systemen und zur Relevanz. Grundwasser - Zeitschrift der Fachsektion Hydrogeologie, 4, 203-212, 2003.

[31] I. Ishii and A. P. Hitchcock. A quantitative exprimental study of th core exited electron states of formamide, formid acid, and formyl flouride. Journal of Chemistral Physics, 87(2), 830-839, 1987.

[32] IUSS-Working-Group-WRB. World reference base for soil resources 2006. World Soil Resources Reports, (103), 2007.

[33] D. P. Jaisi, H. Dong, J. Kim, Z. He, and J. P. Morton. Nontronite particle aggregation induced by microbial Fe(III) reduction and exopolysaccharide production. Clays and Clay Minerals, 55(1), 96-107, 2007.

[34] P. A. C. Jansson, U. Vogt, and H. M. Hertz. Liquid-nitrogen-jet laser-plasma source for compact soft $x$-ray microscopy. Review on Scientific Instruments, 76, 043503, 2005.

[35] A. Jarre, J. Seeger, C. Ollinger, C. Fuhse, C. David, and T. Salditt. X-ray Waveguide Nanostructures - Design, Fabrication and Characterization. Journal of Applied Physics, 101, 054306, 2007.

[36] K. Jasmund and G. Lagaly, editors. Tonminerale und Tone. Steinkopff Verlag Darmstadt, Germany, 1993.

[37] B. Kaulich, D. Bacescu, J. Susini, C. David, E. D. Fabrizio, G.R.Morrison, P. Charalambous, J. Thieme, T. Wilhein, J. Kovac, D. Cocco, M. Salome, O. Dhez, T. Weitkamp, S. Cabrini, D. Cojoc, A. Gianoncelli, U. Vogt, M. Podnar, M. Zangrando, M. Zacchigna, and M. Kiskinova. TwinMic 
- A European Twin X-ray Microscopy Station Commissioned at ELETTRA. In: S. Aoki, Y. Kagoshima, and Y. Suzuki, editors, X-Ray Microscopy, Proceedings of the 8th Intl. Conf., volume 7 of Conference Series, 22-25. Institute of Pure and Applied Physics, Tokyo, 2006.

[38] J. Kirz. Phase Zone Plates for X-Rays and Ectreme UV. Journal of the Optical Society of America, 3, 301-309, 1974.

[39] J. Kirz, C. Jacobsen, and S. Williams. Soft X-ray microscopes and their biological applications. Quarterly Reviews of Biophysics, 28, 33-130, 1995.

[40] C. Knöchel. Anwendung und Anpassung tomographischer Verfahren in der Röntgenmikroskopie. Ph.D. thesis, Georg-August-Universität Göttingen, 2005.

[41] C. Knöchel, S. G. J. Thieme, G. Schneider, W. Bates, and G. Schmahl. Tomographic studies of nontronite particles in aqueos media. In: J. Susini, D. Joyeux, and F. Polack, editors, X-Ray Microscopy 2002, rth Intl. Conf. on X-Ray Microscopy, volume 104 of Journal de Physique IV Proceedings, 425-428. 2003.

[42] A. M. L. Kraepiel, K. Keller, and F. M. M. Morel. A Model for Metal Adsorption on Montmorillonite. Journal of Colloid and Interface Science, 210, 4354, 1999.

[43] G. Lagaly. Reaktionen der Tonminerale. In: K. Jasmund and G. Lagaly, editors, Tonminerale und Tone, 89-167. Steinkopff Verlag Darmstadt, Germany, 1993.

[44] G. Lagaly and H. M. Köster. Tone und Tonminerale. In: K. Jasmund and G. Lagaly, editors, Tonminerale und Tone, 1-32. Steinkopff Verlag Darmstadt, Germany, 1993.

[45] G. Lagaly, O. Schulz, and R. Zimehl. Dispersionen und Emulsionen. Steinkopff Verlag Darmstadt, Germany, 1997.

[46] C. Larabell and M. Le Gros. X-ray tomography generates 3-D reconstruction of the yeast, Saccharomyces cerevisiae, at 60-nm-resolution. Molecular Biology of the Cell, 15, 957-962, 2004. 
[47] J. Lehr. Entwicklung, Charakterisierung und Anwendung von dreidimensionalen Bildgebungsverfahren für die Röntgenmikroskopie. Ph.D. thesis, Georg-AugustUniversität Göttingen, 1997.

[48] J. Maser. Theoretische Ausbreitung der Beugungs- und Abbildungseigenschaften hochauflösender Zonenplatten für die Röntgenmikroskopie. Ph.D. thesis, GeorgAugust-Universität Göttingen, 1994.

[49] D. N. Mastronade. Dual-axis tomography: an approach with alignment methods that preserve resolution. Journal of Structural Biology, 120, 343-352, 1997.

[50] W. Meyer-Ilse, G. Denbeaux, L. Johnson, Bates, A. W., Lucero, and E. Anderson. The High Resolution X-ray Microscope. In: W. Meyer-Ilse, T. Warwick, and D. Attwood, editors, X-Ray Microsocpy, Proceedings 6th Intl. Conf. X-Ray Microscopy, 129-134. American Institute of Physics, Melville, 2000.

[51] A. G. Michette. Optical Systems for Soft X-Rays. Plenum Press, New York, 1986.

[52] G. Mitrea, J. Thieme, P. Guttmann, S. Heim, and S.-C. Gleber. X-ray spectromicroscopy with the scanning transmission X-ray microscope at BESSYII. Journal of Synchrotron Radiation, 15, 26-35, 2008.

[53] G. R. Morrison. X-Ray Microscopy IV. Bogorodskii Pechatnik Publishing, 479484, 1994.

[54] F. Natterer. The mathematics of computerized tomography. Springer-Verlag, Heidelberg, 1986.

[55] U. Neuhäussler. Soft X-ray Spectromicroscopy on Hydrated Colloidal and Environmental Science Samples. Ph.D. thesis, Georg-August-Universität Göttingen, 1999.

[56] B. Niemann, D. Rudolph, and G. Schmahl. Soft X-ray imaging zone plates with large zone numbers for microscopic and spectroscopic applications. Optics Communications, 12.

[57] J. Niemeyer, J. Thieme, P. Guttmann, T. Wilhein, D. Rudolph, and G. Schmahl. Direct imaging of aggregates in aqueous clay suspensions by X-ray microscopy. Progress in Colloid and Ploymer Science, 95. 
[58] J. Niemeyer, J. Thieme, and G. Machulla. X-ray microscopy of soil colloids. In: J. Thieme, G. Schmahl, D. Rudolph, and E. Umbach, editors, X-Ray Microscopy and Spectromicroscopy, II-3 - II-10. Springer-Verlag, Heidelberg, 1998.

[59] C. Ollinger, C. Fuhse, S. Kalbfleisch, R. Tucoulou, and T. Salditt. Object localization with $10 \mathrm{~nm}$ accuracy by $x$-ray phase contrast projection imaging. Applied Physical Letters, 91, 051110, 2007.

[60] D. Y. Parkinson, G. McDermott, L. D. Etkin, M. L. Gros, and C. A. Larabell. Quantitative 3-D imaging of eucaryotic cells using soft X-ray tomography. Journal of Structural Biology, 162, 380-386, 2008.

[61] C. Quitmann, C. David, F. Nolting, F. Pfeiffer, and M. Stampanoni, editors. 9th International Conference on X-Ray Microscopy, volume 186 of Journal of Physics: Conference Series. IOP electronc journals, 2009.

[62] J. Radon. Über die Bestimmung von Funktionen durch ihre Integralwerte längs gewisser Mannigfaltigkeiten. Berichte über die Verhandlungen der Sächsischen Akademie der Wissenschaften zu Leipzig, Math.-Nat. Klasse, 69, 262-277, 1917.

[63] F. Rhoton, M. Römkens, J. Bigham, T. Zobeck, and D. Upchurch. Ferrihydrite influence on infiltration, runoff and soil loss. Soil Science Society of America Journal, 67, 1220-1226, 2003.

[64] G. Schmahl and D. Rudolph. Lichtstarke Zonenplatten als abbildende Systeme für weiche Röntgenstrahlung. Optik, 29, 577-585, 1969.

[65] G. Schmahl, D. Rudolph, P. Guttmann, and O. Christ. Zone plates for X-ray microscopy. In: G. Schmahl and D. Rudolph, editors, Springer series in Optical Sciences, 63-74. Springer-Verlag, Heidelberg, 1984.

[66] G. Schneider. Röntgenmikroskopie mit Synchrotronstrahlung an wässrigen b iologischen Systemen - experimentelle und theoretische Untersuchungen. Ph.D. thesis, Georg-August-Universität Göttingen, 1992.

[67] G. Schneider. High-Resolution X-Ray Microscopy of Radiation Sensitive Material. Cuvillier Verlag, Göttingen, 1999.

[68] G. Schneider, E. Anderson, S. Vogt, C. Knöchel, D. Weiß, M. Le Gros, and C. Larabell. Computed tomography of cryogenic cells. Surface Review \& Letters, 9, 177-183, 2002. 
[69] H. D. Schulz and A. Hadeler, editors. Geochemical Processes in Soil and Groundwater, Measurement - Modelling - Upscaling. GeoProc 2002: Research Report (Forschungsberichte (DFG)). Wiley-VCH Verlag, Weinheim, Germany, 2003.

[70] U. Schwertmann and R. M. Cornell. Iron Oxides in the Laboratory. WILEYVHC, Weinheim, Germany, second edition, 2000.

[71] J. Sedlmair, S.-C. Gleber, H. Zänker, P. Guttmann, S. Heim, and J. Thieme. Characterization Studies of Carbon Nanotubes. submitted.

[72] Soil-Survey-Division-Staff. Soil Survey Manual. Soil Conservation Service. U.S. Department of Agriculture Handbook 18. pdf download, http://soils.usda.gov/technical/manual, 1999.

[73] Soil-Survey-Staff. Soil Taxonomy, A Basic System of Soil Classification for Making and Interpreting Soil Surveys. Soil Conservation Service. U.S. Department of Agriculture Handbook 436. pdf download, http://soils.usda.gov/technical/classification/taxonomy, second edition, 1999.

[74] D. Sparks. Environmental Soil Chemistry. Academic Press, San Diego, USA, 1995.

[75] G. Sposito. The Chemistry of Soils. Oxford University Press, Oxford, UK, 1998.

[76] F. J. Stevenson. Humus Chemistry: Genesis, Composition, Reactions. John Wiley \& Sons, Inc. New York, USA, second edition, 1994.

[77] F. J. Stevenson and M. A. Cole. Cycles of Soil - Carbon, Nitrogen, Phosphorus, Sulfur, Micronutrients. John Wiley \& Sons, Inc. New York, USA, second edition, 1999.

[78] J. Stöhr. NEXAFS Spectroscopy. Springer, 1996.

[79] H. Stollberg, M. Pokorny, and H. M. Hertz. A vacuum-compatible wetspecimen chamber for compact X-ray microscopy. Journal of Microscopy, 226(1), 71-73, 2007.

[80] L. Strüder, H. Bräuninger, M. Meier, P. Predehl, C. Reppin, M. Sterzik, J. J. Trümper, P. Cattaneo, D. Hau, G. Lutz, K. Schuster, A. Schwarz, E. Kendziorra, R. Staubert, E. Gatti, A. Longoni, M. Sampietro, V. Radeka, P. Rehak, S. Rescia, P. F. Manfredi, W. Buttler, P. Holl, 
J. Kemmer, U. Prechtel, and T. Ziemann. The MPI/AIT X-ray Imager (MAXI) - High speed pn-CCDs for X-ray detection. NIM A, 288, 227-235, 1990.

[81] J. Susini, D. Joyeux, and F. Polack, editors. X-Ray Microscopy 2002, 7th Intl. Conf. on X-Ray Microscopy, volume 104 of Journal de Physique IV Proceedings. EDP Sciences, Les Ulis, 2003.

[82] P. A. C. Takman, H. Stollberg, G. A. Johannson, A. Holmberg, M. Lindblom, and H. M. Hertz. High-resolution compact X-ray microscopy. Journal of Microscopy, 226, 175-181, 2007.

[83] K. H. Tan. Environmental Soil Science. Marcel Dekker, Inc., New York, USA, second edition, 2000.

[84] J. Thieme, S. Gleber, G. Mitrea, and P. Guttmann. Microscopy and spectromicroscopy with soft X-ray. Optics and Precision Engineering, 12, 1887-1885, 2007.

[85] J. Thieme, P. Guttmann, J. Niemeyer, G. Schneider, C. David, B. Niemann, D. Rudolph, and G. Schmahl. Röntgenmikroskopie zur Untersuchung von wässrigen biologischen und kolloidchemischen Systemen. Nachr. Chem. Tech. Lab, 40, 562-563, 1992.

[86] J. Thieme, I. McNulty, S. Vogt, and D. Paterson. X-ray spectromicroscopy A tool for environmental sciences. Environmental Science and Tecnolology, 41(20), 1027, 2007.

[87] J. Thieme and J. Niemeyer. Interaction of colloidal soil particles, humic substances and cationic detergents studied by X-ray microscopy. Progress in Colloid and Polymer Science, 111, 193-201, 1998.

[88] J. Thieme, G. Schneider, and C. Knöchel. X-ray tomography of a microhabitat of bacteria and other soil colloids with sub-100 nm resolution. Micron, 34, 339-344, 2003.

[89] J. Thieme, J. Sedlmair, S.-C. Gleber, M. Bertilson, O. von Hofsten, P. Takman, and H. Hertz. High-resolution imaging of soil colloids in aqueous media with a compact soft X-ray microscope. In: 9th International Conference on X-Ray Microscopy, volume 186 of Journal of Physics: Conference Series (IOP), 012107. 2009. 
[90] O. Vormoor. Aggregation kolloidaler Teilchen: Ein Vergleich von Röntgenmikroskopie und Streumethoden. Ph.D. thesis, Georg-August-Universität Göttingen, 2002.

[91] Y. Wang, C. Jacobsen, J. Maser, and A. Osanna. Soft X-ray microscopy with a cryo scanning transmission X-ray microscope: I. Instrumentation, imaging and spectroscopy. Journal of Microscopy, 197, 68-79, 1999.

[92] Y. Wang, C. Jacobsen, J. Maser, and A. Osanna. Soft X-ray microscopy with a cryo scanning transmission X-ray microscope: II. Tomography. Journal of Microscopy, 197, 80-93, 1999.

[93] K. A. Weber, L. A. Achenbach, and J. D. Coates. Microorganisms pumping iron: anaerobic microbial iron oxidation and reduction. Nature Reviews Microbiology, 4, 752-764, 2006.

[94] D. Weiß. Computed tomography based on cryo X-ray microscopic images of unsectioned biological specimens. Ph.D. thesis, Georg-August-Universität Göttingen, 2000.

[95] D. Weiß, G. Schneider, B. Niemann, P. Guttmann, D. Rudolph, and G. Schmahl. Computed tomography of cryogenic biological specimens base. Optik, 84, 185-197, 2000.

[96] U. Wiesemann. The Scanning Transmission X-Ray Microscope at BESSYII. Ph.D. thesis, Georg-August-Universität Göttingen, 2003. RQE920, RRE000, RRG000.

[97] U. Wiesemann, J. Thieme, P. Guttmann, F. R., S. Rehbein, B. Niemann, D. Rudolph, and G. Schmahl. First results of the new scanning transmission $X$ ray microscope at BESSY II. In: J. Susini, D. Joyeux, and F. Polack, editors, $X$ Ray Microscopy 2002, rth Intl. Conf. on X-Ray Microscopy, volume 104 of Journal de Physique IV Proceedings, 95-98. EDP Sciences, Les Ulis, 2003.

[98] H. Wolter. Spiegelsysteme streifenden Einfalls als abbildende Optiken für Röntgenstrahlen. Annalen der Physik, (6), 1952. 


\section{Acknowledgements}

This work would not have been possible without the amazing support of dear friends, colleagues, and family. I thank all of them with all my heart.

First of all, I would like to thank Jürgen Thieme for the supervision of this work as project leader who is well experienced in soft X-ray microscopy and environmental science. I am very grateful for the opportunity of having worked at four different X-ray microscopes. The cooperation was supported by a valuable friendship.

As head of institute, Tim Salditt created a fruitfull and very pleasant working atmosphere. I deeply appreciate his vivid interest and constructive remarks on my thesis and his support for any scientific or organisational issue. I thank him for giving me the opportunity of doing my doctorate in the Institute for X-ray Physics under his supervision.

I still benefit from Prof. Schmahl's enthusiasm to X-ray microscopy and therefore I thank him again for the supervision of my diploma thesis.

Enthusiasm for environmental science was transmitted in an incomparable lecture by Prof. Niemeyer. Since then, dynamics is not just a word for me when it is related to soil colloids. I also very much appreciate the revealing discussions on the x-ray micrographs of the clay and soil samples.

For the great support at the X-ray microscopes I am grateful to Peter Fischer and Weilun Chao from the XM-1, Hans Hertz, Michael Bertilson and Olov von Hofsten from the CSXM, Peter Guttmann and Stefan Heim from the STXM, Alessandra Gianoncelli and Burkhard Kaulich and from the TwinMic, and Christian Peth and Thomas Mann from the LLG, Göttingen (although the results from the LLG experiments are not presented here).

Thanks to Peter Nieschalk und Karsten Wulf for the realisation of the two-piece aixle and the adaption of STXM sample plate.

The organisation of travel expenses and whatever-necessary was perfectly managed by Sabine Balder und Christina Emser. Thank you for all the help there! 
All the IRP colleagues together established a great team spirit - keep our D-cafe running for spontaneous visits from alumni!

The second half of my doctorate was enriched by Julia Sedlmair, with whom I then shared the office and a notable number of hotel and guest house rooms. She became a dear friend to me and a great colleague for beam times and conferences!

Many thanks to Sebastian Kalbfleisch for saving me with computer support even at weekends on the low cost of just one cake per off-hour assignment.

I would also like to thank the members of our deanery, the faculty, and equal opportunity office: Besides more work, I got an inspiring insight to organisational structures.

I thank Alexander Rack for his friendship and discussions about X-ray microscopy. Furthermore, amoung all dear friends, I would like to thank explicitely Julia Richter and Stephan Sehmisch for supporting me and for offering Florian an emotional valve during the last weeks of me writing my thesis.

Many, many thanks to Monika, Horst and Konstantin August for all your encourage and cordial support given to me as an affiliated member in your very special family circle.

Gudrun is not only my older sister and mathematical confederate from the childhood, but also the IDL Queen for me - thanks for the support in programming, which would not have been affortable in times before the telephone flat rates! I also thank my other sisters Johanna and Dorothee for exactly that kind of support only sisters can give. Furthermore, you enriched our family by my charming nephews Robert and Niklas, who are already heartbreaking the women at my institute, and my cute niece Beeke. Papa, I thank you heartily for providing me many different ways into a diverse world. This was strongly and boundlessly completed by my beloved mum, who is always with me.

Finally, I thank Florian August for all the purest love, strength, and inspiration he is giving me in every single moment. I dedicate this work to our future. 


\section{Lebenslauf}

Name

Geburtsdatum:

Geburtstort:

Staatsangehörigkeit :

$1984-1990$

1990 - 1994

$1994-1997$

$1997-1998$

$1998-2004$

$2004-2005$

Seit September 2005
Sophie-Charlotte Gleber

25.11.1977

Wolfenbüttel

Deutsch

Grundschule und Orientierungsstufe Lüne, Lüneburg Johanneum, Lüneburg

Otto-Hahn-Gymnasium Göttingen

Au-pair Aufenthalt in Madrid, Spanien

Physik-Studium an der Georg-August-Universität Göttingen

Diplomarbeit bei Prof. Dr. G. Schmahl:

"Untersuchungen zur Stereomikroskopie mit Röntgenstrahlen"

Hilfswissenschaftlerin am Institut für Röntgenphysik, Georg-August-Universität Göttingen

Wissenschaftliche Angestellte am Institut für Röntgenphysik, Georg-August-Universität Göttingen

Promotion bei Prof. Dr. T. Salditt:

"Soft X-ray stereo microscopy for investigation of dynamics and elemental distribution of colloidal systems from the environment" 